\title{
Active Investment: Evaluating the Benefits and Costs of Investing in Active Travel in the Urban New Zealand Context.
}

\author{
Mark Grams
}

Environmental Studies 593

2014

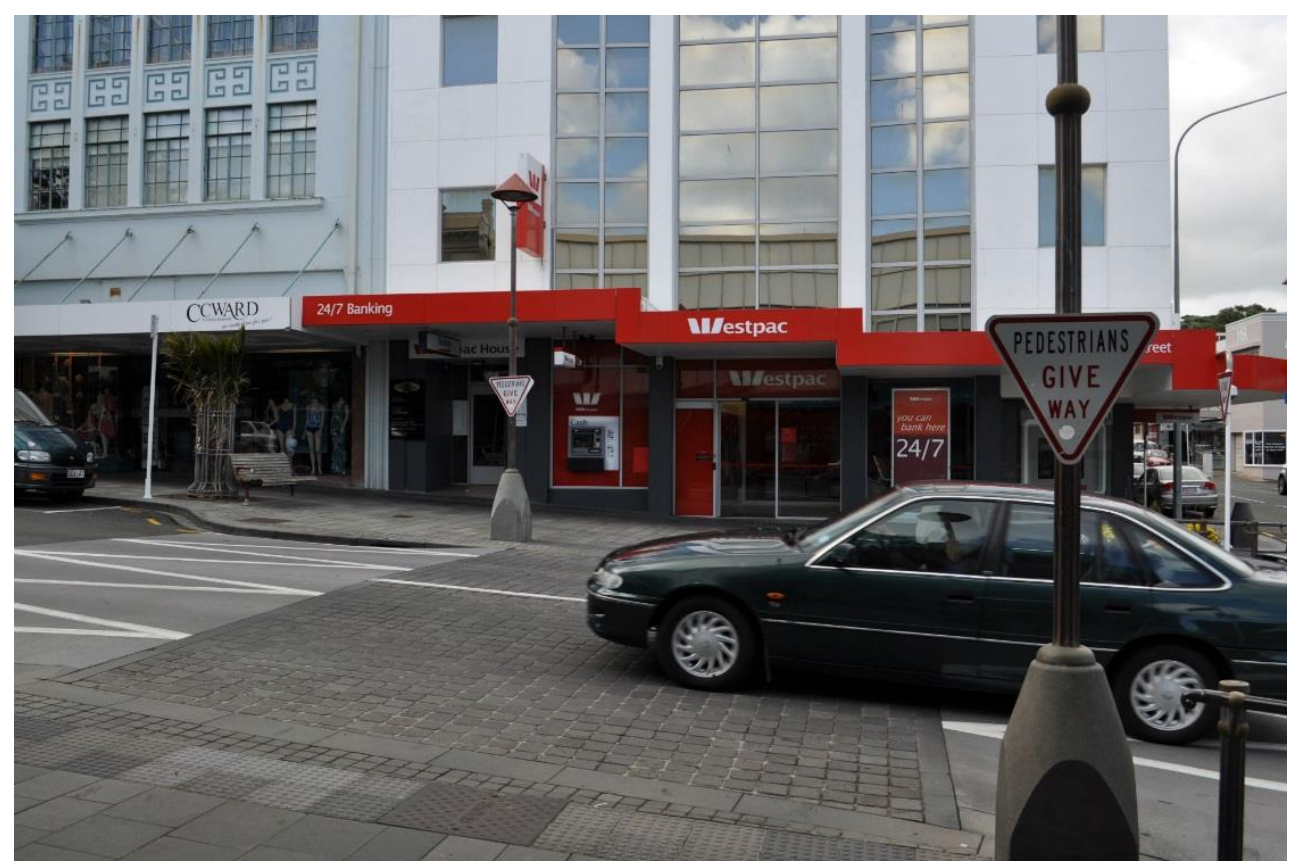

"Pedestrians give way": An indicative street sign in New Plymouth? Photo credit: Mark Grams.

A 90 point thesis submitted to Victoria University of Wellington, as partial fulfilment of requirements for the degree of Master of Environmental Studies

School of Geography, Environment and Earth Sciences

Victoria University of Wellington

June, 2014 



\begin{abstract}
Increased uptake of active travel is widely acknowledged to promote improved health, environmental and community outcomes. Although active travel participation rates in New Zealand compare favourably to other Anglo-Saxon countries, many northern European countries report active travel rates that are many fold higher.

In response to declining rates of active travel across New Zealand, the New Zealand Transport Agency created the Model Communities Programme. The goal of this programme was to boost walking and cycling rates in two demonstration cities, Hastings and New Plymouth, by improving walking and cycling infrastructure, as well as funding promotional and educational initiatives.

This study evaluates the effectiveness of the Model Communities Programme. This evaluation includes a quantitative appraisal in the form of a cost-benefit analysis. In addition to evaluating the economic efficiency of the programme, this study provides qualitative analysis derived from user intercept surveys and interviews with district council project managers to further assess the effectiveness of the intervention.

This study has found the Model Communities Programme to be cost-beneficial under several evaluative frameworks. In addition to this, the qualitative analysis has also presented largely positive feedback from both users and project managers. This study concludes that the Model Communities Programme has indeed boosted active travel in the demonstration cities, and that similar interventions are likely to be effective at promoting active travel across urban New Zealand.
\end{abstract}

Key words: active travel, walking, cycling, economic evaluation, cost-benefit analysis, Model Communities Programme 


\section{Acknowledgements}

This research reflects the effort of several individuals.

I would like to thank Ralph Chapman, my supervisor, for his guidance and careful reading. Thanks also to Philippa Howden-Chapman, Michael Keall and the New Zealand Centre for Sustainable Cities for their financial and practical assistance towards my research. Thanks also to the following individuals for their key contributions to my study: Owen Mata from Hastings District Council; Carl Whittleston and Kathryn Scown from the New Plymouth District Council; Tom Pettit, Ed Randall, Jean Beetham and Dalice Sim from Victoria University of Wellington. My appreciation also goes out to the walkers and cyclists in Hastings and New Plymouth, several of whom enthusiastically provided feedback for this study; your contributions were the highlight of my research.

I would also like to thank all my other friends and family, both near and far, for their endless words of encouragement and support throughout this postgraduate study. Much gratitude goes to my partner Susan for being supportive in more ways than I could ever possibly thank her for. 


\section{Table of contents}

List of figures

List of tables .. $\mathrm{V}$

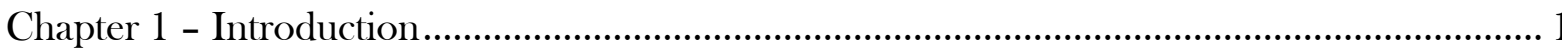

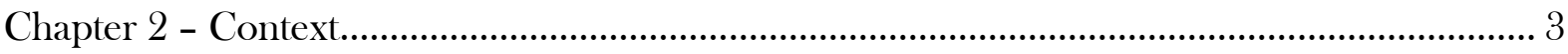

2.1 Active travel, health and greenhouse gas emissions in New Zealand ...................... 3

2.2 Active travel, transport policy and the Model Communities Programme............... 9

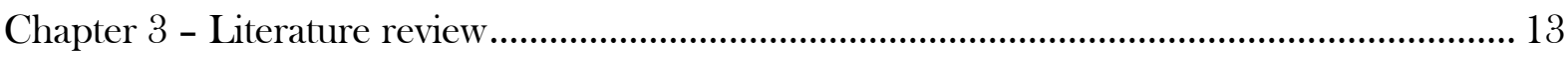

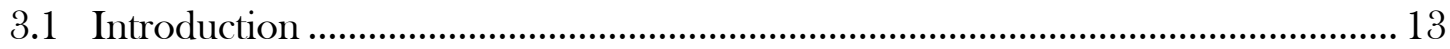

3.2 Background on economic evaluation of active travel interventions....................... 13

3.3 A review of cost-benefit analyses of active travel interventions .............................. 16

3.4 NZTA's 2013 EEM CBA framework for active travel evaluation.......................... 20

3.5 Critiquing past active travel economic evaluation practice .................................... 24

3.6 Critique of $\mathrm{CBA}$ as an evaluation tool for active travel interventions ..................... 25

3.7 Alternative methods of evaluating active travel interventions.................................. 28

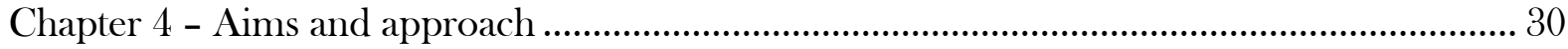

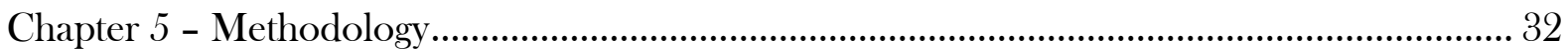

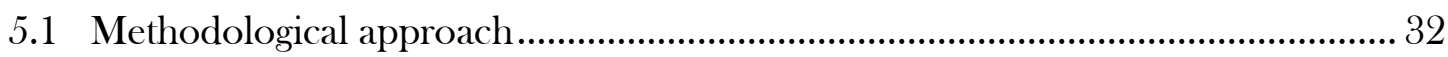

5.2 CBA of the Model Communities Programme: analysis of costs ........................... 33

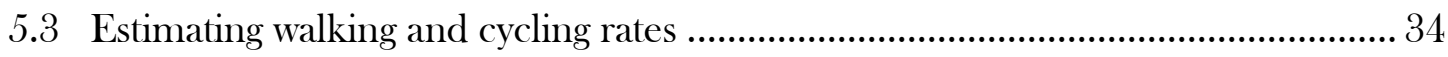

5.4 Benefit analysis of the MCP .................................................................................. 41

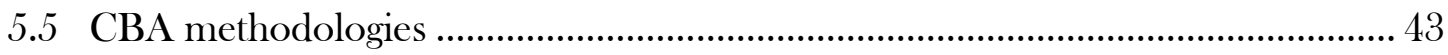

5.6 Analysis of Model Communities Programme users' perceptions of benefits ....... 50

Chapter 6 - Results of the CBA for the Model Communities Programme

6.1 CBA \#1 (based on NZTA 2010 EEM framework)............................................... 55

6.2 CBA \#2 (based on NZTA 2013 EEM framework)............................................ 57

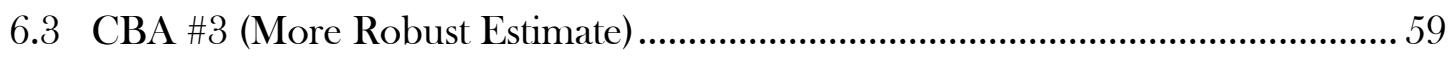

6.4 CBA \#4 (Optimistic Estimate) ................................................................................ 61

Chapter 7 - Analysis of Model Community Programme users' perceptions of benefits.

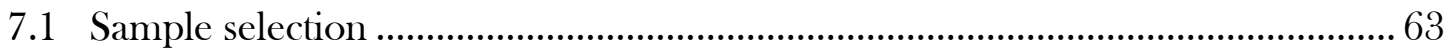

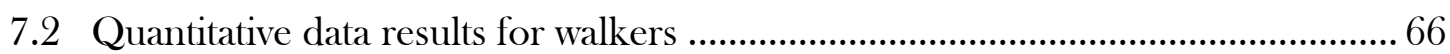

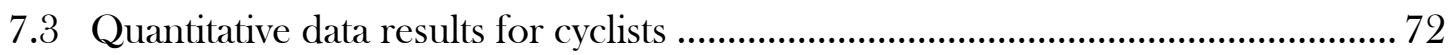

7.4 Qualitative data results for walkers and cyclists ...................................................... 78

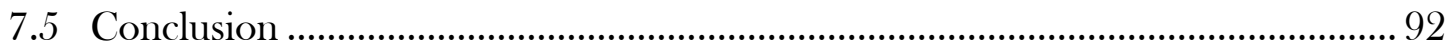


Chapter 8 - Model Communities Programme project managers' feedback.

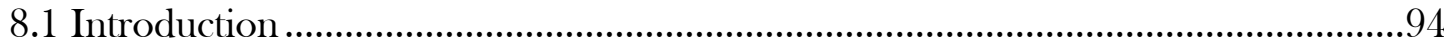

8.2 Project managers critical feedback of the interventions ............................................95

Chapter 9 - Discussion

9.1 Discussion of results from the CBA evaluations of the MCP ............................ 101

9.2 Discussion of intercept surveys and project manager interviews ......................... 110

9.3 Strengths and limitations of this research ........................................................ 119

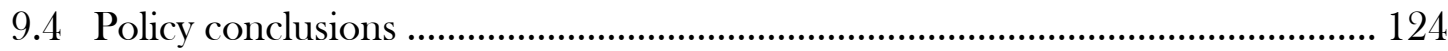

9.5 Recommendations for further research.......................................................... 126

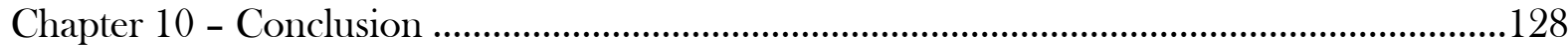

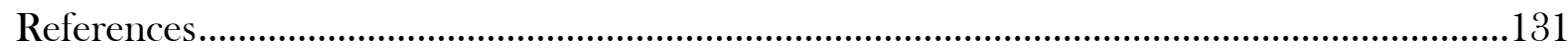

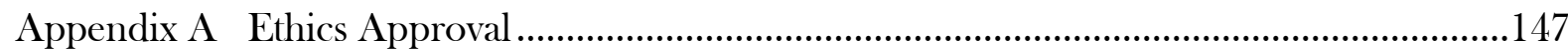

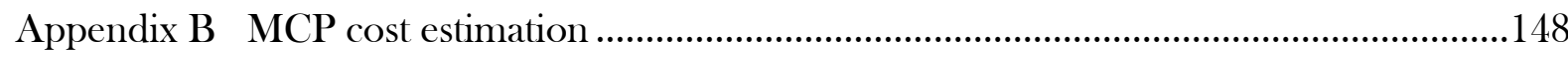

Appendix C Active travel growth estimates ..............................................................................149

Appendix D UK Department for Transport Carbon Prices .....................................................151

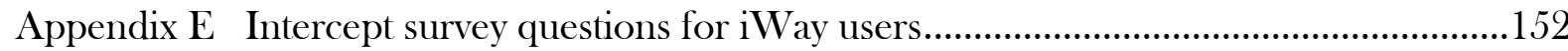

Appendix F Project manager interview questions ..............................................................157 


\section{List of figures}

Figure 1: Weekly time spent travelling per person (ages five and over) ....................................... 3

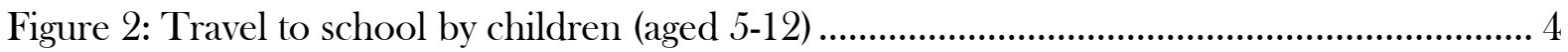

Figure 3: Travel to school by secondary school students (aged 13-17) ...................................... 4

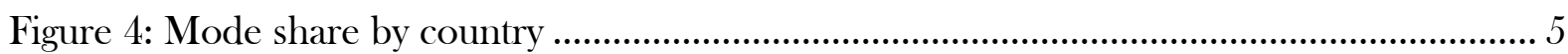

Figure 5: Physically active, by neighbourhood deprivation and sex............................................ 5

Figure 6: Total greenhouse gas emissions in selected OECD countries ................................... 8

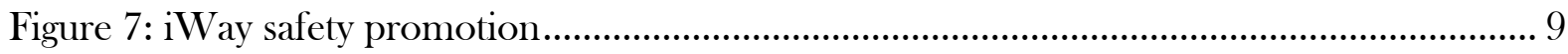

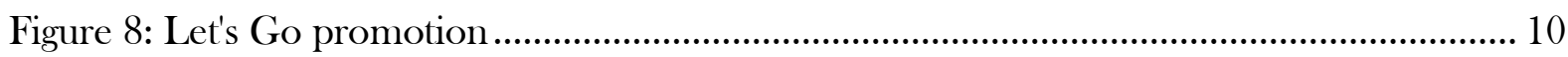

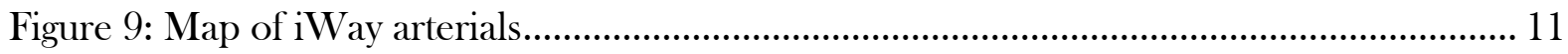

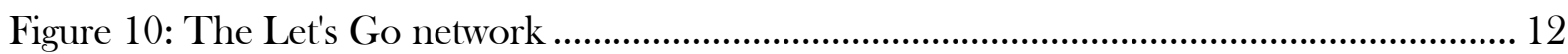

Figure 11: Six cycling demonstration town results.............................................................. 17

Figure 12: How many kilometres could be built for $\$ 20$ million, per $\mathrm{km}$ costs?......................... 30

Figure 13: \% change of mean distances walked pre and post intervention periods ................... 37

Figure 14: \% change of mean distances cycled pre and post intervention periods ..................... 38

Figure 15: Distribution of benefits for CBA \#1, medium estimate, no control ......................... 55

Figure 16: Distribution of benefits for CBA \#1, medium estimate, with control ....................... 56

Figure 17: Distribution of benefits for CBA \#2, medium estimate, no control ..........................5 57

Figure 18: Distribution of benefits for CBA \#2, medium estimate, with control ....................... 58

Figure 19: Distribution of benefits for CBA \#3, medium estimate, without control ................. 59

Figure 20: Distribution of benefits for CBA \#3, medium estimate, with control...................... 60

Figure 21: Distribution of benefits for CBA \#4, medium estimate, no control ........................ 61

Figure 22: Distribution of benefits for CBA \#4, medium estimate, with control ....................... 62

Figure 23: MCP intercept surveys, respondents surveyed by district council............................ 64

Figure 24: 2006 Census population by sex for Hastings and New Plymouth districts ............... 64

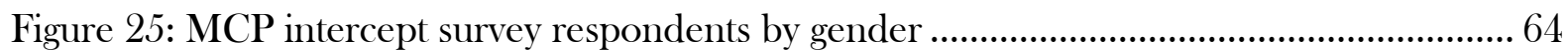

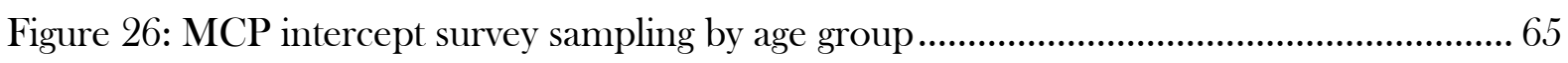

Figure 27: 2006 Census respondents by ethnicity for Hastings and New Plymouth.................. 66

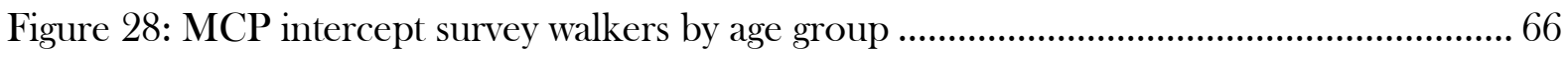

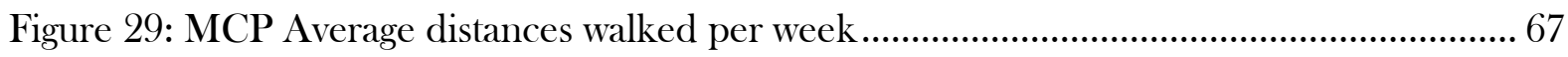

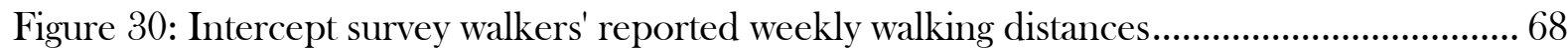

Figure 31: Frequency of \#1 rankings for walkers per criteria 2013 ......................................... 70

Figure 32: Frequency of \#6 rankings for walkers per criteria 2013 ........................................ 71

Figure 33: Walkers' willingness to pay in Hastings and New Plymouth................................... 71

Figure 34: MCP intercept survey cyclists by age group .............................................................. 72

Figure 35: Respondents regularly cycling in 2013 reported 2009 cycling distances................... 72

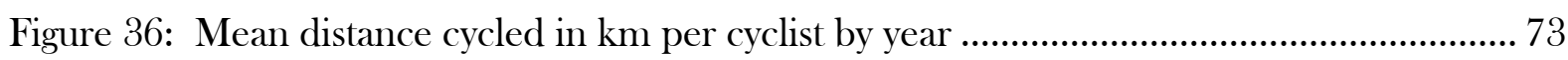

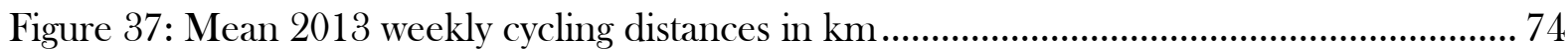

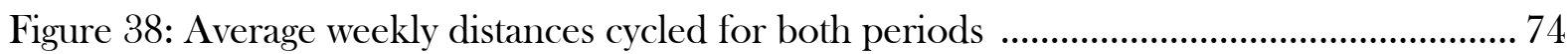

Figure 39: Frequency of \#1 rankings for cyclists per criteria 2013 .......................................... 77

Figure 40: Frequency of \#6 rankings for cyclists per criteria 2013 ............................................. 77

Figure 41: Cyclists' willingness to pay in Hastings and New Plymouth ...................................... 78

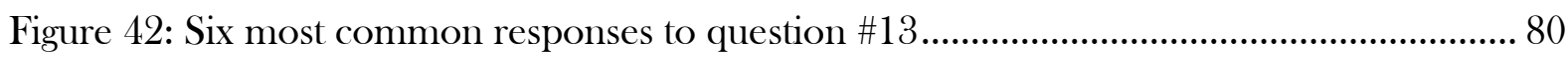

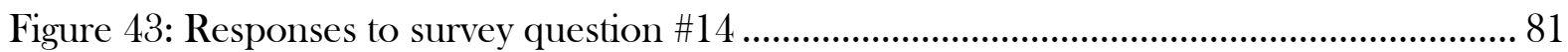




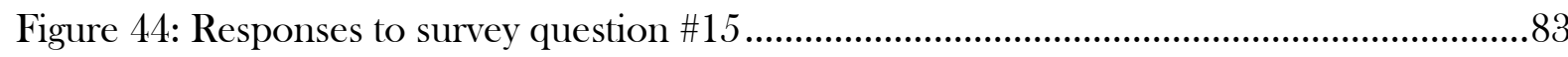

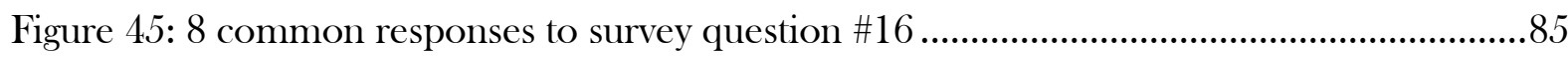

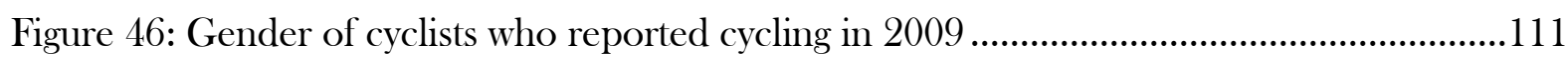

Figure 47: Gender of cyclists who reported cycling in 2013 .................................................11

Figure 48: Walkers' perceptions pre and post intervention .......................................................114

Figure 49: Mean 2013 ratings of cycling facilities in Hastings and New Plymouth...................114

Figure 50: Frequency of \#1 rankings for walkers and cyclists per criterion in 2013 ................115

Figure 51: Frequency of \#6 rankings for walkers per criterion in 2013 ..................................116 


\section{List of tables}

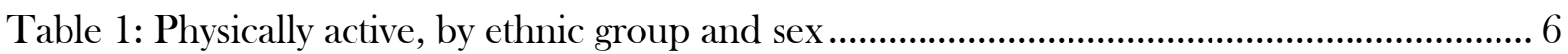

Table 2: Obesity, by ethnic group and sex, from Ministry of Health ......................................... 7

Table 3: Projections of emission and removals by sector and by year ........................................ 8

Table 4: Key figures and results for 3 investment plans for bicycling in Porltand ..................... 17

Table 5: Macmillan's CBA of proposed cycling plans in Auckland ......................................... 19

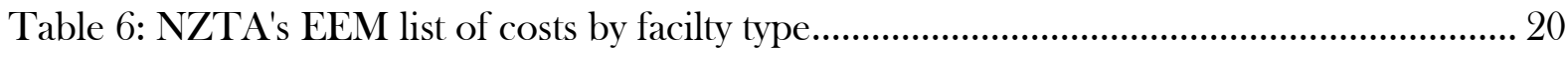

Table 7: EEM behavioural values of time for vehicle occupants................................................. 21

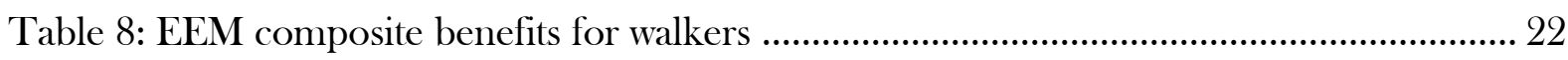

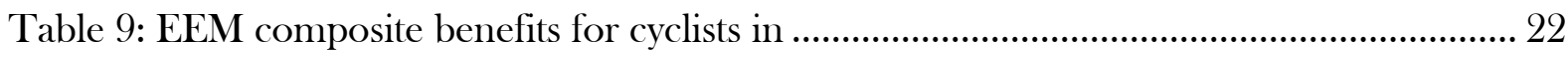

Table 10: EEM relative benefits for different types of cycle facilities........................................ 23

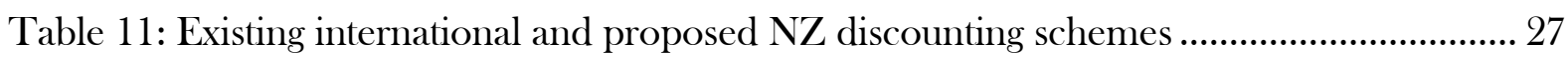

Table 12: Cycling infrastructure funding and cycling overall mode share ................................... 30

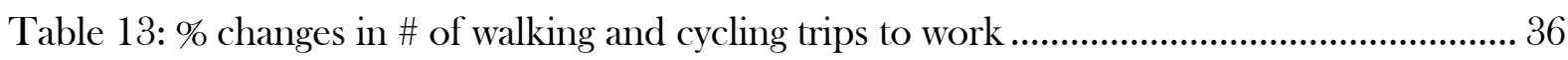

Table 14: Walking growth estimates based on 2001-2013 Census travel to work data ............. 38

Table 15: Cycling growth estimates based on 2001-2013 Census travel to work data ............... 39

Table 16: CBA \#1 without control, using NZTA EEM 2010 guidelines .................................. 55

Table 17: CBA \#1 with control, using NZTA EEM 2010 guidelines .........................................56

Table 18: CBA \#2, without control, based on NZTA 2013 EEM framework ........................... 57

Table 19: CBA \#2, with control, based on NZTA 2013 EEM framework................................ 58

Table 20: CBA \#3, without control, more robust estimate .....................................................59

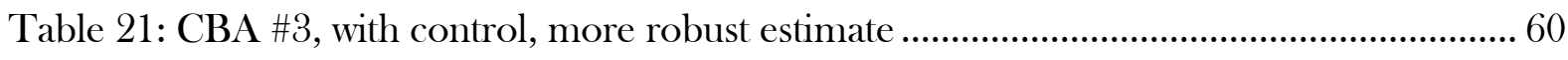

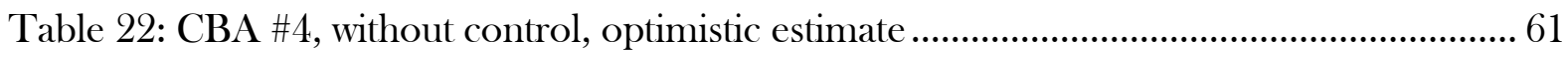

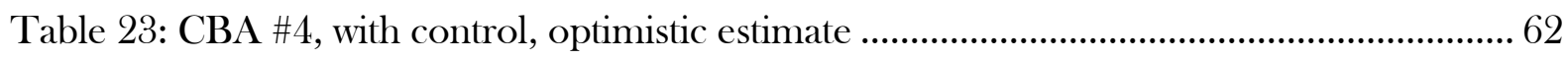

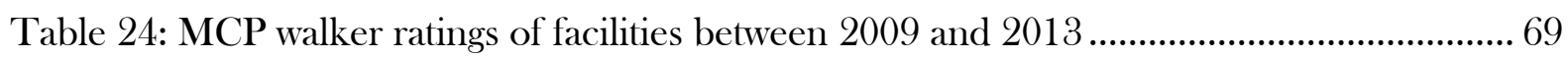

Table 25: Walkers' ranking of criteria in Hastings and New Plymouth........................................ 70

Table 26: Cyclists' ratings of criteria in Hastings and New Plymouth........................................ 75

Table 27: Cyclists' 2013 rankings of criteria in Hastings and New Plymouth ............................ 76

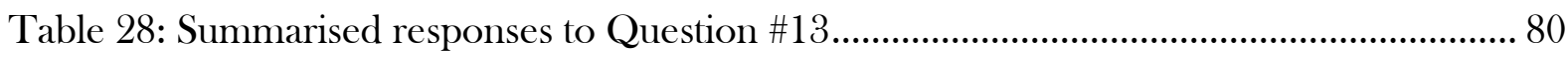

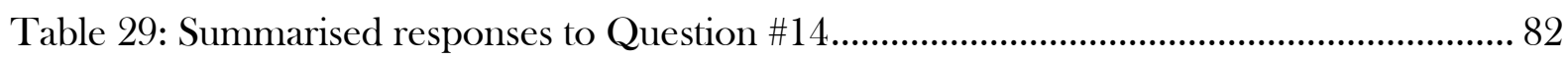

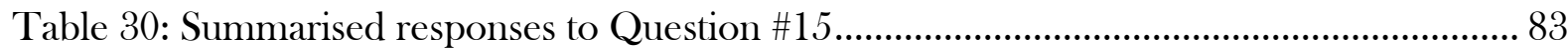

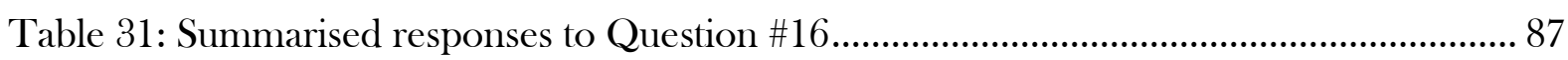

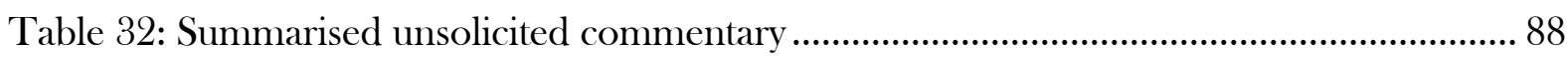

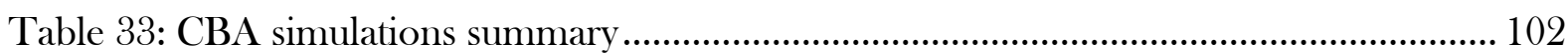

Table 34: Comparing NZTA's 2013 EEM to Krizek et al.'s models ...................................... 107 



\section{Chapter 1 - Introduction}

Increased uptake of active travel (AT) ${ }^{1}$ in the urban environment is widely recognised by experts in urban planning and public health as providing numerous benefits to society.

Direct benefits commonly associated with increased uptake of active travel include but are not limited to: improved health outcomes and reduced external costs of motor vehicle use which can include reduced congestion, parking, local emissions, greenhouse gas emissions, and vehicle operating costs. There is also an increasing amount of research which suggests that greater participation in active travel has other indirect benefits, including improved community outcomes in the form of increased social interaction/cohesion, reduced feelings of severance and improved liveability/walkability of urban areas.

Despite these increasingly accepted benefits associated with active travel, there are wide disparities in active travel participation rates across countries in the developing world, and in some cases, between urban areas within the same country. Some developed countries such as the Netherlands, Denmark and Germany have the highest modal shares for walking and cycling in the world, while Anglo-Saxon countries such as the US, the UK, Canada and Australia have active modal shares that are a fraction of the aforementioned northern continental European countries'. There are numerous theories behind these disparities including: differing urban forms, social preferences or even political bias towards certain modes of transport, as well as variance in levels of investment in interventions that support active travel modes. This latter relationship is often supported by data suggesting that the countries that have invested the most per-capita in active travel interventions typically report the highest levels of walking and cycling. This trend is seen in cities in the same country. Approximately $1 \%$ of US residents report cycling to work on a daily basis according to US census data. This rate is up to ten times higher in cities like Portland, Oregon and Davis, California, both cities which have made substantial investments in cycling infrastructure.

Census and household travel survey data in New Zealand suggest that walking and cycling rates are significantly lower than in many Northern European nations, although slightly higher than in the US and Australia. New Zealand residents are by and large dependent on motor vehicles for their daily travel needs. National investment in active travel is a

\footnotetext{
${ }^{1}$ Transport by active means, most commonly walking and cycling
} 
very small fraction of the national road transportation budget, far lower than modal shares for both walking and cycling.

One might assume that increasing national investment in walking and cycling interventions is the most effective way to boost New Zealanders' participation in active travel. However, this to date is a largely untested hypothesis, especially for significant scale AT investment. The city of Wellington, according to the more recent census and New Zealand Household Travel Survey (NZHTS) data, has seen dramatic increases in cycling with no substantial investments in active travel infrastructure between the 2006 and 2013 censuses. At the same time, NZHTS data suggest that cycling is in decline for small cities $(<100,000)$ in New Zealand, despite overall increases in cycling for large cities $(>100,000$ residents).

In 2010, the New Zealand Transport Agency (NZTA), implemented the Model Communities Programme (MCP), a nationally funded initiative investigating whether or not a significant scale investment targeting walking and cycling is likely to boost participation for these modes of active travel. National funding was provided for two district councils, Hastings and New Plymouth, with both councils contributing funds to the intervention as well. This MCP provides a unique opportunity to investigate the effectiveness of significant-scale targeted investment for active travel interventions within the urban New Zealand context, notably for small New Zealand cities which collectively are experiencing diminishing rates of walking and cycling. 


\section{Chapter 2 - Context}

\subsection{Active travel participation, health and greenhouse gas emissions in the New Zealand national context}

In the past three decades, New Zealand has seen a declining rate of active travel (AT)

participation at the national level. Data from the New Zealand Ministry of Transport (2009) show that over three sampling periods from 1989 to 2008, the average amount of time New Zealanders spent driving a car or as a car passenger has steadily increased while the amount of time New Zealanders spent walking or cycling has decreased (Figure 1).

A report by Genter \& NZ Transport Agency (2009) uses work travel data from the Census to illustrate the downward trend in active mode shares across regions. Wellington and Nelson are the only regions that do not follow this trend with Wellington increasing its active modal share

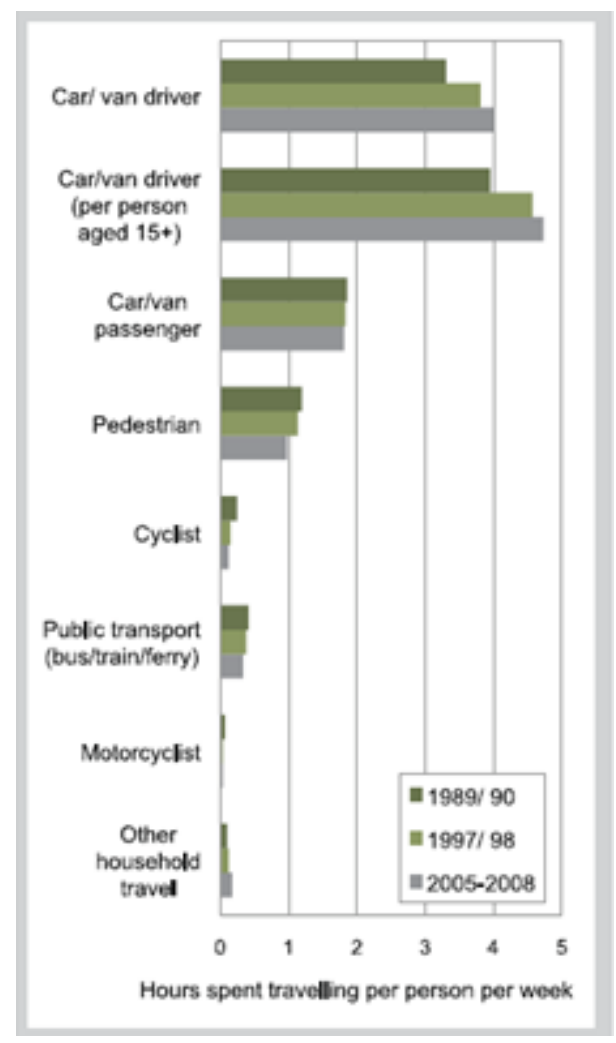

Figure 1: Weekly time spent travelling per person (ages five and over), from Ministry of Transport (2009, p.5) across the three periods whereas Nelson's rates have remained relatively static.

In Sizing up the City: Urban form and transport in New Zealand, Keall, Chapman, \& Howden-Chapman (2010) highlight an alarming trend of diminishing rates of children's active mode travel share to school. According to Ministry of Transport (2009) data, walking and cycling rates to school for children (aged 5-12 years old, see Figure 2) have dropped from just over $50 \%$ in 1989 to less than $30 \%$ in 2008 . In this same time period, teenagers' (aged 13-17 years old, see Figure 3) total walking and cycling travel shares to school have dropped from just under $50 \%$ to about $25 \%$, with cycling alone dropping from approximately $18 \%$ in 1989 to $5 \%$ in 2008 . Rates of traveling as a car passenger have increased for both age groups, with rates of driving holding about the same for the older age group. The MoT 2009-2012 New Zealand Household Travel Survey estimates that pedestrian trips accounted for $16 \%$ of total modal share while cycling trips accounted for $1.4 \%$, together giving a 17.4\% share for active modes (Ministry of Transport, 2013a). 


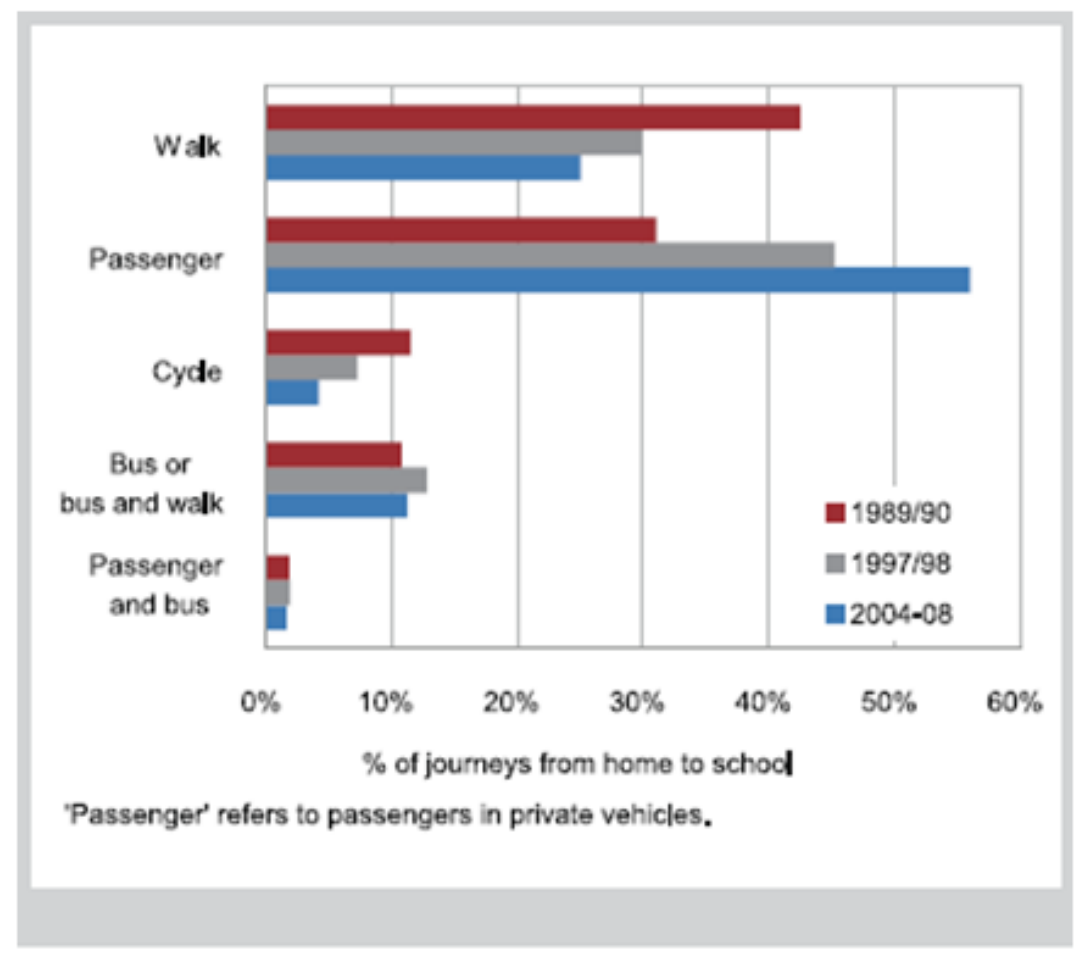

Figure 2: Travel to school by children (aged 5-12), from Ministry of Transport (2009, p.17)

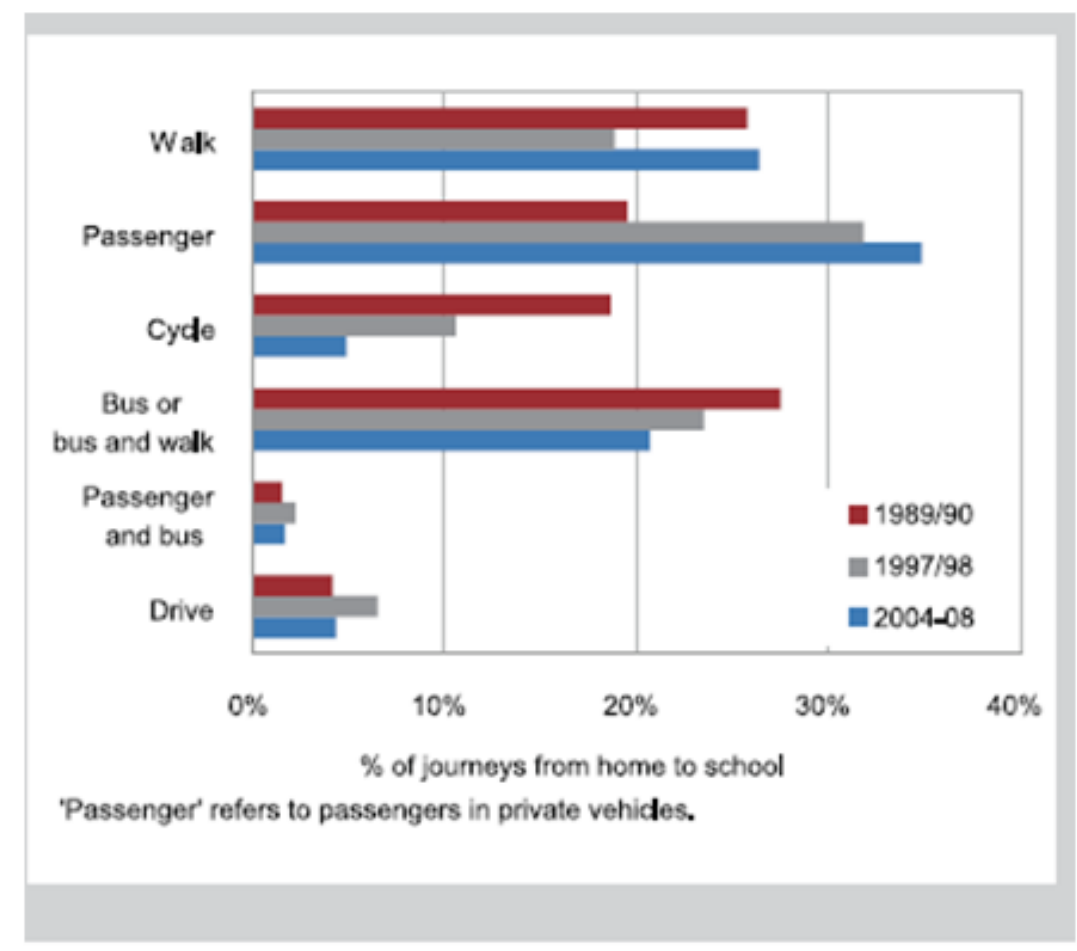

Figure 3: Travel to school by secondary school students (aged 13-17), from Ministry of Transport (2009, p.17) 
In comparison to other countries, New Zealand's active travel share ranks higher than that of Australia, the US and Canada, though lower in comparison to most European nations as seen in Figure 4 (Litman, 2013a).

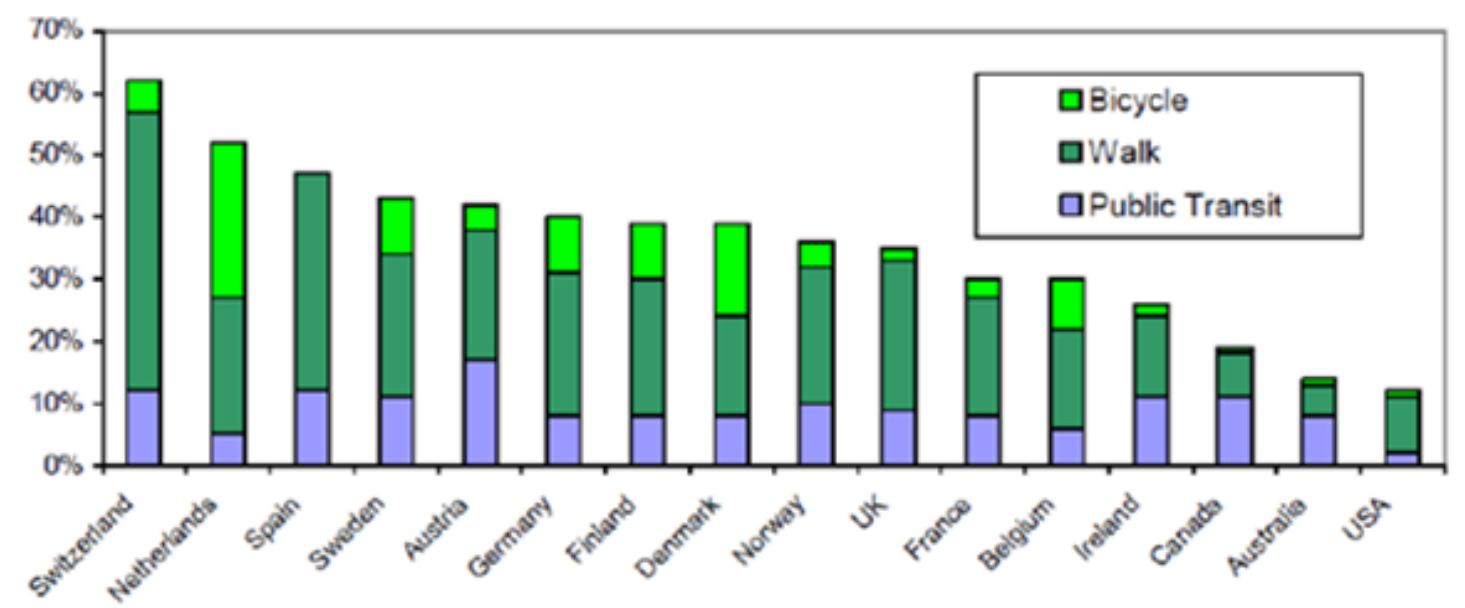

Figure 4: Mode share by county, from Litman (2013a, p.7)

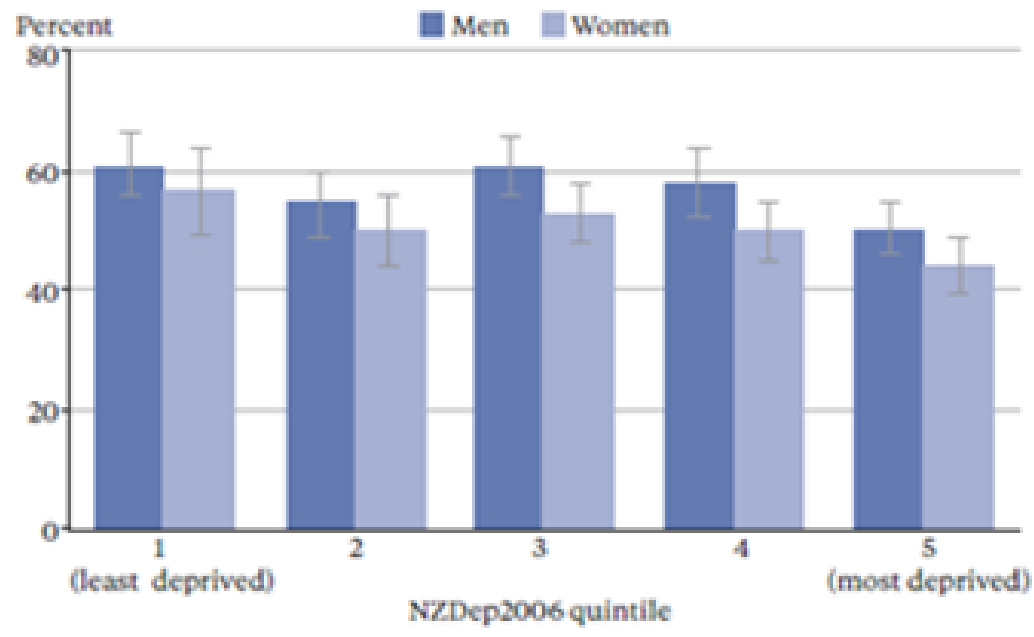

Figure 5: Physically active, by neighbourhood deprivation and sex, from Ministy of Health (2012, p.32) 
In terms of health in the New Zealand context, the New Zealand Health Surveys undertaken by the Ministry of Health (MOH) in 2011-2012 found that almost half $(\sim 46 \%)$ of New Zealand adults did not report being sufficiently active, defined as an adult exercising for 30 minutes per day, at least five days a week (2012). The 2011-2012 New Zealand Health Surveys also report that the number of sufficiently active adults has remained relatively unchanged since the 2002-2003 New Zealand Health Surveys. The 2006-2007 Ministry of Health New Zealand Health Survey estimated that nearly one in ten adults $(9.9 \%)$ were classified as sedentary, defined as less than 30 minutes of physical activity per week, with the remaining portion of the population $(\sim 36 \%)$ being classified as insufficiently active. The 2011-2012 New Zealand Health Survey data also show that both women and residents of high deprivation communities are less likely than men and residents of low deprivation communities to report being sufficiently active (Figure 5).

The 2011-2012 survey also suggests that Pacific Islanders and Asians are comparatively less sufficiently active than Maori and Europeans (Table 1). The Ministry of Health's New Zealand Health Survey data also show obesity has increased for both boys and girls since the 2006-2007 survey (Table 2), with Maori and Pacific Islander children showing three to four times the rate of obesity as Europeans and Asians $(17 \%$ \& $23 \%$ rates of childhood obesity compared to $6 \%$ \& 7\%) in 2011-2012.

\begin{tabular}{|c|c|c|c|c|c|}
\hline & & Total & Men & Women & $\begin{array}{r}\text { Estimated } \\
\text { number }\end{array}$ \\
\hline \multirow[t]{5}{*}{ Percent (\%) } & Total NZ & 54 & 57 & 51 & $1,905,000$ \\
\hline & Msori & 57 & 61 & 54 & 254,000 \\
\hline & Pacific & 46 & 53 & 40 & 95,000 \\
\hline & Asian & 39 & 39 & 39 & 147,000 \\
\hline & European/Other & 56 & 59 & 52 & $1,555,000$ \\
\hline \multirow{3}{*}{$\begin{array}{l}\text { Adjusted rate ratios (comparing } \\
\text { each ethnic group with people } \\
\text { not in that ethnic group) }\end{array}$} & Malori & 1.0 & 1.0 & 1.0 & \\
\hline & Pacific & $0.8^{\circ}$ & 0.9 & $0.8^{*}$ & \\
\hline & Asian & $0.7^{*}$ & $0.6^{\circ}$ & $0.7^{\circ}$ & \\
\hline
\end{tabular}

Table 1: Physically active, by ethnic group and sex, from Ministry of Health (2012, p.32) 


\begin{tabular}{|c|c|c|c|c|c|}
\hline & & Total & Boys & Girls & $\begin{array}{r}\text { Estimated } \\
\text { number' }\end{array}$ \\
\hline \multirow[t]{5}{*}{ Percent (\%) } & Total NZ & 10 & 10 & 10 & 91,000 \\
\hline & Mãori & 17 & 19 & 14 & 38,000 \\
\hline & Pacific & 23 & 23 & 24 & 33,000 \\
\hline & Asian & 7 & 9 & 5 & 6,000 \\
\hline & EuropeanVOther & 6 & 6 & 7 & 39,000 \\
\hline \multirow{3}{*}{$\begin{array}{l}\text { Adjusted rate ratios (comparing } \\
\text { each ethnic group with children } \\
\text { not in that ethnic group)? }\end{array}$} & Mãori & $2.1^{*}$ & $2.8^{*}$ & $1.6^{*}$ & \\
\hline & Pacific & $3.1^{*}$ & $3.0^{\circ}$ & $3.2^{*}$ & \\
\hline & Asian & 0.7 & 0.9 & 0.5 & \\
\hline
\end{tabular}

Table 2: Obesity, by ethnic group and sex, from Ministy of Heallth (2012b, p.27)

A $2013 \mathrm{MoH}$ report entitled "Health Loss in New Zealand" has calculated Disability Adjusted Life Years (DALYs) for common medical conditions in New Zealand (Tobias, Turley \& Ministry of Health, 2013). DALYs represent the number of years lost due to premature mortality or disability. Medical conditions related to inactivity can include cardiovascular disease (CVD), cancer, diabetes and depression (Ministry of Health, 2012a). Cancer and CVD each accounted for 17.5\% of total DALYs in New Zealand, with diabetes (3\%) and depression (5.3\%) also reporting significant numbers of DALYs (Tobias et al., 2013).

New Zealand transportation habits continue to contribute to greenhouse gas emissions worldwide. According to a Ministry for the Environment (MFE) 2007 report, New Zealand's vehicle ownership has tripled since the 1950s; in 2005, New Zealand ranked $5^{\text {th }}$ in the per capita rate of vehicle ownership within the Organization of Economic Cooperation and Development (OECD). In $2005 \mathrm{New}$ Zealand also ranked $5^{\text {th }}$ in the OECD for total greenhouse gas emissions per capita, which includes $\mathrm{CO}_{2}$ emissions (Figure 6), (Ministry for the Environment, 2009). Data from a 2013 MfE report (Table 3) show that although the agricultural sector currently accounts for the highest quantity of greenhouse gas emissions (35.2 million tonnes, $\mathrm{CO}_{2}$ equivalent), the energy \& transport sector contribution is only a little lower (31.7 million tonnes, $\mathrm{CO}_{2}$ equivalent). Given New Zealand's currently high level of car dependence, modal shift to alternative modes of transportation such as walking and cycling have the potential to play a key role in reducing national greenhouse gas emissions. 


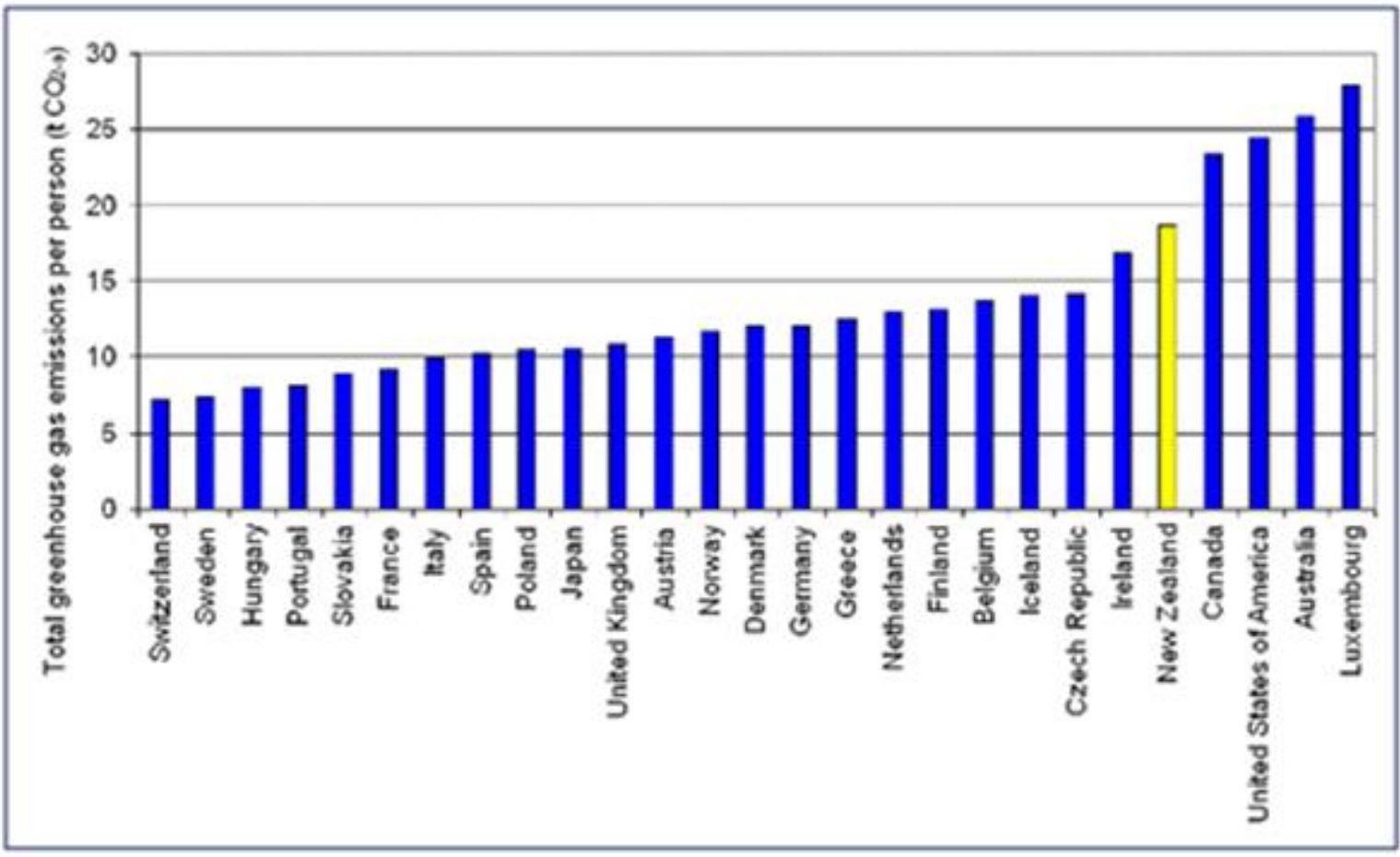

Figure 6: Total greenhouse gas emissions (tonnes of carbon dioxide equivalent) per person in 2005 for selected OECD countries, from Ministry for the Environment (2009)

\begin{tabular}{l|r|r|r|r|r|r}
\multicolumn{1}{c}{} & \multicolumn{7}{c}{ Emissions and removals (millions of tonnes of $\mathrm{CO}_{2}$ equivalent) } \\
\cline { 2 - 7 } & 2008 & 2009 & 2010 & 2011 & 2012 & Total \\
\hline Energy (including Transport) & 34.3 & 31.6 & 31.3 & 31.0 & 31.7 & 159.9 \\
\hline Industrial processes and solvents & 4.3 & 4.3 & 4.8 & 5.5 & 4.4 & 23.3 \\
\hline Agriculture & 33.3 & 33.5 & 33.7 & 34.4 & 35.2 & 170.2 \\
\hline Waste & 2.1 & 2.0 & 2.0 & 2.0 & 2.0 & 10.1 \\
\hline Forestry & & & & & & \\
\hline Net removals & -15.3 & -15.5 & -15.9 & -15.6 & -14.9 & -77.2 \\
\hline Deforestation & 2.8 & 2.7 & 2.5 & 2.8 & 3.5 & 14.3 \\
Gross removals & -18.1 & -18.2 & -18.3 & -18.4 & -18.4 & -91.5 \\
\hline Total & 58.8 & 56.0 & 56.0 & 57.2 & 58.4 & 286.3 \\
\hline
\end{tabular}

Table 3: Projections of emission and removals by sector and by year, from Ministry for the Environment $(2013$, p.8) 


\subsection{Active travel, transport policy and the Model Communities Programme}

In 2005 the governing Labour party released a policy document entitled "Getting there on foot, by cycle" (Minister of Transport, 2005) recognizing the downward trends of walking and cycling rates in New Zealand. This document outlined a vision of "A New Zealand, where people from all sectors of the community walk and cycle for transport and enjoyment" as well as a strategic goal of improving community environments and transport systems to support pedestrians and cyclists (Minister of Transport, 2005, p.10). In 2008, the Ministry of Transport (under a Labour-led government) released the New Zealand Transport Strategy 2008 outlining the following vision: "People and freight in New Zealand in New Zealand have access to an affordable, integrated, safe, responsive and sustainable transport system" (Ministry of Transport, 2008, p.5). The New Zealand Transport Strategy 2008 also set several ambitious goals to achieve by 2040 including: increasing active modal share to $30 \%$, increasing modal share for public transport to $7 \%$ and halving per capita greenhouse gases from domestic transport (Ministry of Transport, 2008, p.5-6).

In "Getting there - on foot, by cycle", the Minister of Transport (2005) proposed the Model Communities Programme, an intervention designed to promote walking and cycling in a few select cities. In 2010, NZTA selected proposals from Hastings and New Plymouth District Councils (out of 22 total proposals) to become part of the Model Communities Programme, an intervention giving both district councils access to NZTA funds to improve walking and cycling infrastructure and education/promotion programmes (Dance, 2012). From 2010-2012, NZTA provided funding for improved infrastructure for New Plymouth (NZ\$ 3.7 million) and Hastings (NZ\$ 3.6 million), as

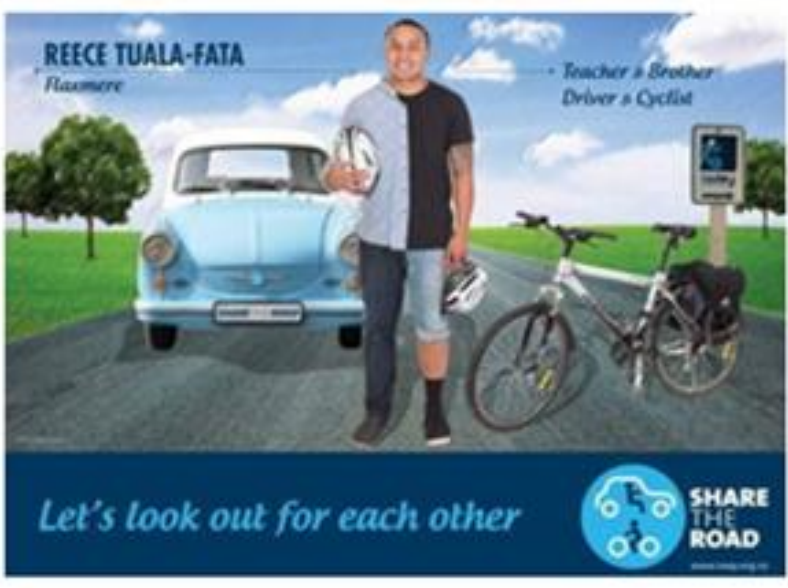

Figure 7: i Way safety promotion, from Dance (2012) well as a combined investment for education and promotion of NZ\$ 1.5 million (Dance, 2012). Both district councils budgeted an estimated NZ\$6.5 million in total (including central government funds) for their interventions, with both councils providing about $30 \%$ of the costs of the intervention (NZTA, 2013c). 
The Hastings District Council branded its intervention “iWay”. iWay's focus has been connecting Hastings with the surrounding residential communities of Flaxmere, Havelock North, Whakatu and Clive with safe routes comprising both off-road shared pathways and marked on-road cycle lanes, as seen in Figure 9 (Dance, 2012). Under iWay, a total of 50 $\mathrm{km}$ of on-road cycle lanes have been marked and $10 \mathrm{~km}$ of Separated bicycle facilities have been constructed (NZTA, 2013c). iWay has invested heavily in marketing and promotion campaigns to normalise perceptions of walking and cycling within the Hastings area and has also provided in-school cycling education programmes and other community promotional events (NZTA, 2013c). iWay aims to achieve a $20 \%$ active modal share by 2020 (NZTA, 2013c).

New Plymouth District Council branded its intervention programme "Let's Go". The infrastructure component of "Let's Go" centred largely around improving surface quality and connectivity of existing shared pathways as well as providing marked cycle lanes within the CBD and connecting neighbourhoods, as seen in Figure 10 (Dance, 2012). The Coastal Walkway, the most popular feature of New Plymouth's active travel network, existed before the MCP; however, it has been extended and linkages between other walkways have been improved (NZTA, 2013c). In total, $5 \mathrm{~km}$ of additional shared pathways have been built and $40 \mathrm{~km}$ of on-road painted cycle lanes have been added in New Plymouth (Dance, 2012). Let's Go has placed a strong emphasis on in-school education programmes as well as some workplace travel programmes and community promotional events (NZTA, 2013c). Data from the 2006 census on travel to work and school showed that in 2006 walking had a 7\% modal share while cycling had a $3 \%$ modal share in New Plymouth (NZTA, 2013c). Let's Go aims to double both walking and cycling shares by 2016 (NZTA, 2013c).

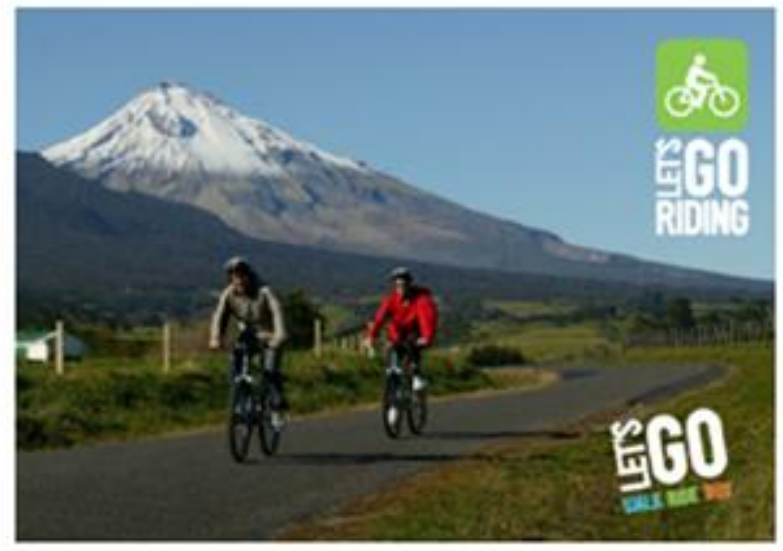

Figure 8: Let's Go promotion, from Dance (2012)

Funding for the original MCP was provided under the 2009-2012 National Land Transport Programme (NLTP) (NZTA, 2009b). Subsequently, NZTA set aside 
additional funding for both Hastings and New Plymouth within the 2012-2015 NLTP; this falls outside the original MCP design (Dance, 2012). It is also worth noting that under the 2012-2015 NLTP, nationally funded investments in walking and cycling projects are budgeted at NZ $\$ 79$ million, with another estimated NZ\$ 80 million for walking and cycling projects tied to other national roading projects such as the Roads of National Significance (NZTA, 2012). Comparing this NZ\$159 million investment in walking and cycling projects to the overall 2012-2015 NLTP budget, projected spending on walking and cycling initiatives represent about 1.3\% of total NLTP 2012-2015 funding, and half of this is tied to national roading projects. When comparing walking and cycling's 2012-2015 NLTP funding share to the 2009-2012 NLTP, the percentage of national funding allocated to walking and cycling facilities is projected to increase from about $1.0 \%$ to $1.3 \%$ of the total NLTP budget (NZTA, 2009b). However, conditions attach to the receipt of funding which means that outturns may well vary from indicative projections.

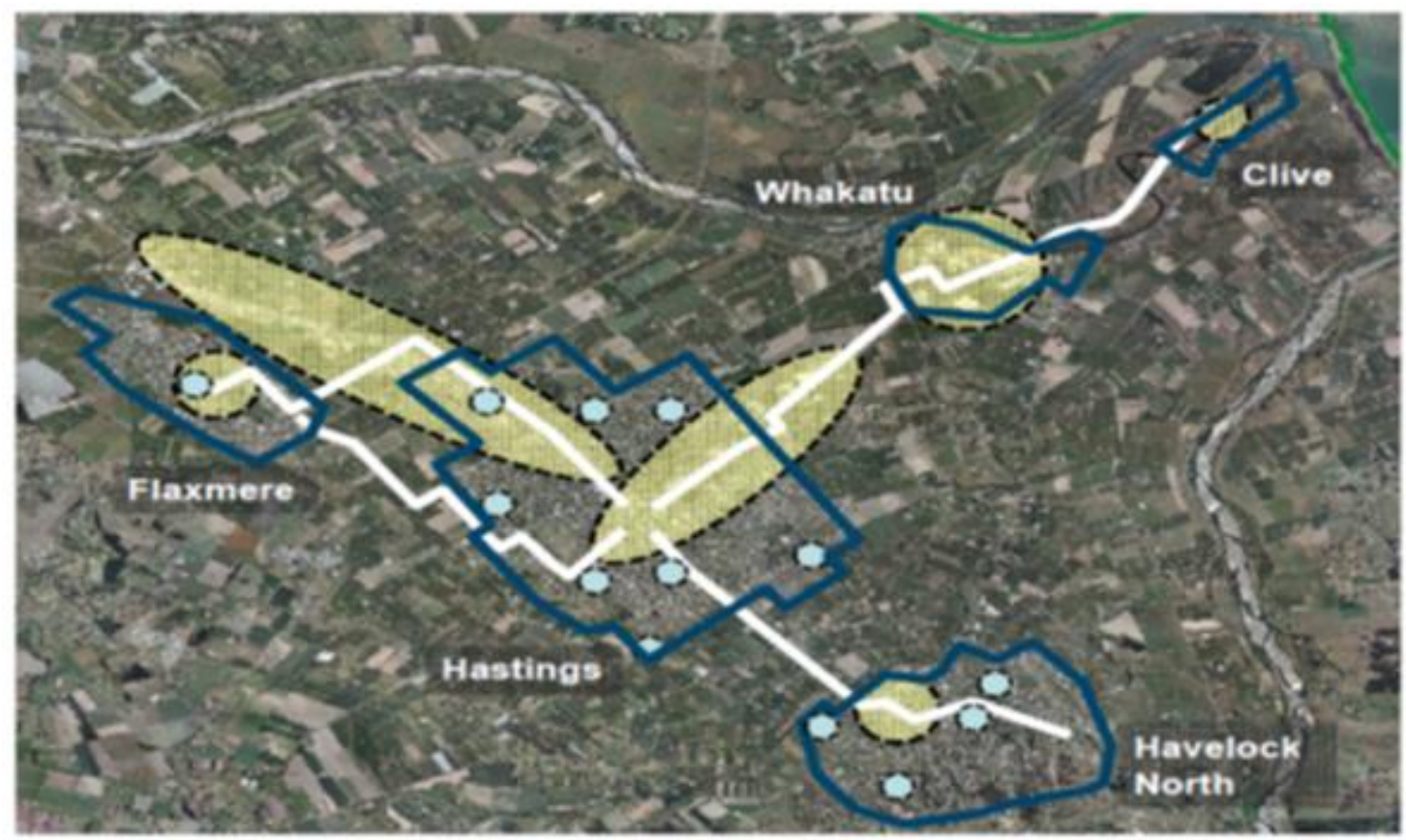

Figure 9: Map of $i$ Way atterials, from Dance (2012) 


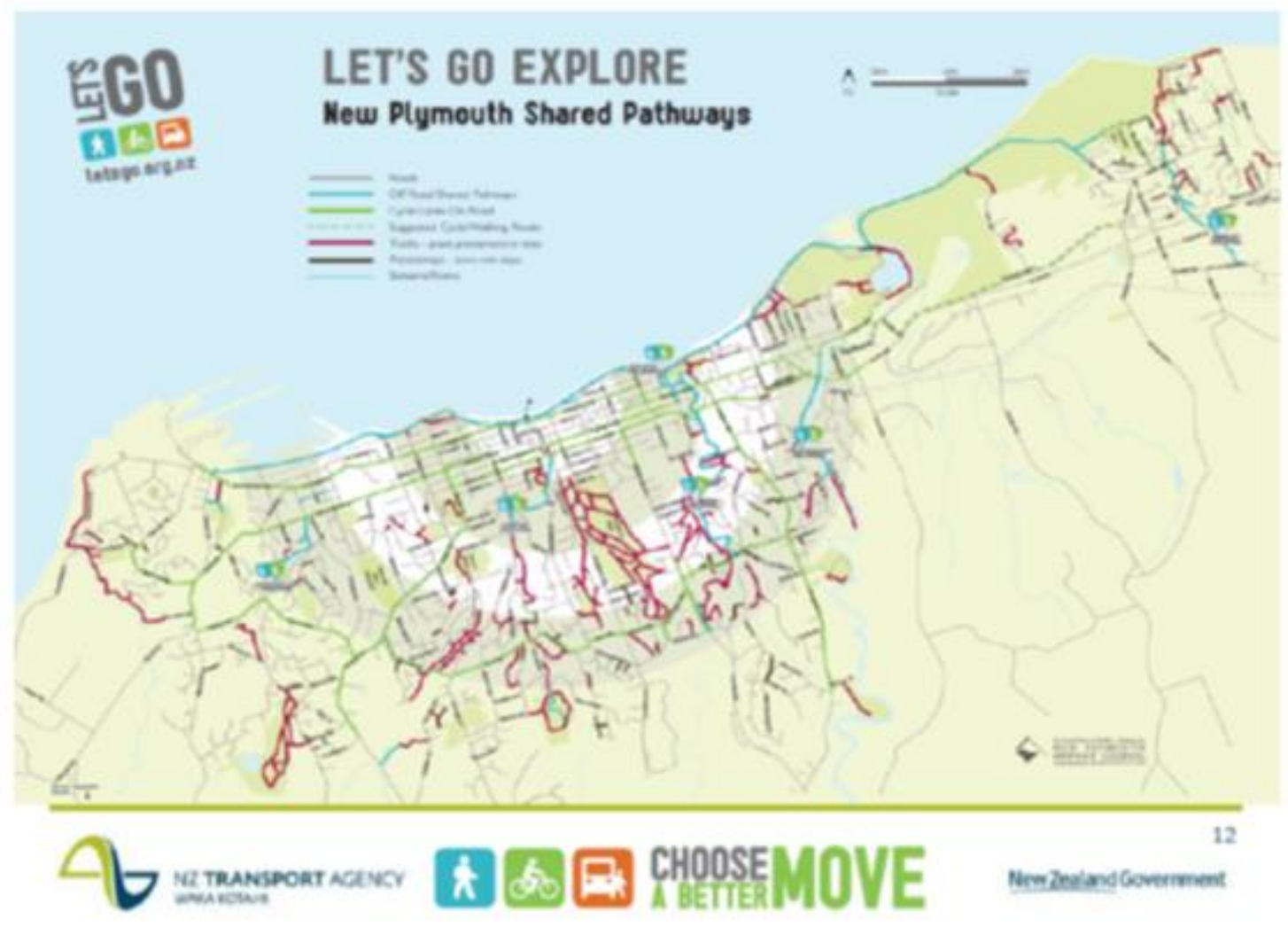

Figure 10: The Let's Go network, from Dance (2012) 


\section{Chapter 3 - Literature review}

\subsection{Introduction}

This chapter will explore the practice of economic evaluation of active travel (AT) ${ }^{1}$ interventions. It will specifically review literature pertaining to: costs and benefits of AT interventions; analysis of previous AT economic evaluations, particularly cost-benefit analysis (CBA); analysis of the New Zealand Transport Agency's (NZTA) 2013 Economic Evaluation Manual (EEM); analysis and critique of AT economic evaluation practice; and alternative methods of evaluating AT interventions.

\subsection{Background on economic evaluation of active travel interventions}

In New Zealand, transportation projects that receive national funds are evaluated through a standardised cost-benefit analysis (CBA) framework set out in the New Zealand Transport Agency's (NZTA) Economic Evaluation Manual (EEM). This process allows a proposed project's potential economic merits to be compared with those of other possible projects aiming to achieve similar objectives. The ultimate goal of this process is to ensure best value for money for transportation investments (Damart \& Roy, 2009; NZTA, 2013a; Wilson \& Cope, 2011). In New Zealand and a few other countries in the world, active travel initiatives are also subjected to CBA. CBA in itself is a simple idea: the costs of a project are compared to expected benefits, and expressed usually as a benefit-cost ratio (BCR). BCRs greater than 1:1 (i.e. where expected benefits exceed costs) are generally seen as being cost-beneficial, or economically efficient. While determining transportation project costs is generally a straightforward procedure, calculating benefit can be considered a problematic and convoluted process (Cavill, Kahlmeier, Rutter, Racioppi, \& Oja, 2008; Litman, 2013a; SQW, 2007). This is especially true in evaluating benefits of AT initiatives (Bauman et al., 2007; Börjesson \& Eliasson, 2012; Cavill et al., 2008; Genter \& NZ Transport Agency, 2009; Saelensminde, 2004). AT projects can either provide new or improved infrastructure designed to facilitate a better environment for walkers and cyclists, or they may simply promote or educate potential users to better use existing infrastructure, or both.

${ }^{1}$ most commonly refers to walking and cycling 


\subsubsection{Analysis of costs associated with active travel initiatives}

Evaluation of AT intervention costs generally include the costs of an intervention's inputs, including all capital, and educational/promotional components. Under the New Zealand Transport Agency's (NZTA) 2013 Economic Evaluation Manual (EEM), evaluation of walking and cycling initiatives should include: construction costs, maintenance costs, operating costs, funding gap (for projects that generate revenue, this represents the shortfall that local and/or national government must cover), net land cost (if new land must be purchased) and planning/design costs (NZTA, 2013a).

\subsubsection{Analysis of benefits associated with active travel initiatives}

AT projects can provide a wide array of benefits. Health, for example, has long been recognised as a substantial benefit of increased AT modal share as a result of AT investment. In recent years the World Health Organisation (WHO), a worldwide authority on global health issues, has developed the Health Economic Assessment Tool (HEAT) in order to provide a platform for assessing monetised health benefits resulting from AT interventions (Kahlmeier \& The World Health Organization Regional Office for Europe, 2013). HEAT has come after the call from some transport and public health academics and policy makers for more robust and consistent frameworks for evaluating expected health benefits from AT initiatives (Dora, Phillips, WHO Regional Office for Europe, 2000; Litman, 2004). The HEAT framework accounts for mortality savings as a result of increased uptake of walking and cycling, but does not as yet include other direct benefits of AT modal shift such as morbidity savings, road traffic reduction benefits (including air/noise pollution), improved safety benefits and improved community liveability benefits (Rutter et al., 2013).

Other benefits such as improved safety for AT users are significant and often undervalued in AT CBA (Hopkinson \& Wardman, 1996; Pettit, 2013; Saelensminde, 2004), but unlike for health there does not appear to be any internationally recognised framework for evaluating improved safety benefits for AT in CBA.

AT investment is also recognised for providing various benefits through motor vehicle savings including congestion savings (Dekoster, Schollaert, European Commission, \& Directorate-General for Environment, 1999; Litman, 2013a), greenhouse gas savings 
(Forsyth \& Krizek, 2010; Giles-Corti, Foster, Shilton, \& Falconer, 2010; Harwatt, Jopson, Muir, Page, \& Tight, 2010) and motor vehicle ownership and vehicle operating cost savings (Litman, 2013a; Minister of Transport, 2005).

According to some authors, another benefit of modal shift to AT is increased opportunity for economic development. This can include decreased absenteeism among employees who become more physically active (Mills, Kessler, Cooper, \& Sullivan, 2007; Pronk et al., 2004; SQW, 2007). Some literature suggests that increased investment in AT initiatives can encourage cycle tourism within cities and regions (Beeton, 2003; Beierle, 2011; Minister of Transport, 2005), while cycle tourism advocates Roca \& Crawford (2013) argue that cycle tourists bring significant amounts of revenue to smaller cities and towns as their mode of transport constrains them to smaller distances travelled per day. Litman (2013a) suggests that increased foot traffic from walkways/cycleways will bring additional consumer spending to businesses that are close to improved walking and cycling facilities in urban areas. A study by Beetham (2014) of an inner-city Wellington street found around $70 \%$ of respondents travelled to this street by a means other than private motor transport.

Provision of AT interventions can provide numerous other benefits to local communities. Roading infrastructure that promotes increased AT modal share allows for accrual of social capital in local communities as users can interact in a more intimate manner with their surrounding environment (Blanco et al., 2009; Boyce, 2010; Leyden, 2003; Yeates, 1999), thereby reducing community severance and barrier effects caused by motor vehicle orientated infrastructure (Blanco et al., 2009; Boyce, 2010; Litman, 2013a; Saelensminde, 2004). Giles-Corti (2010), in the tradition of Jacobs (1961), argues that increased foot traffic can reduce neighbourhood crime by allowing for "natural surveillance" within local communities. Provision of AT infrastructure is thought to make urban transportation more accessible and equitable across many vulnerable strata of society including: the socioeconomically disadvantaged (Banister \& Gallent, 1999; Boyce, 2010; Ogilvie et al., 2011; Yeates, 2001), the elderly (Giles-Corti et al., 2010; Yeates, 1999), the physically handicapped (Giles-Corti et al., 2010; Litman, 2013b) and children (Whitzman, Worthington, \& Mizrachi, 2010; Yeates, 1999). All these community benefits associated with AT interventions are receiving an increasing amount of attention in economic evaluation of transportation projects, although effectively incorporating them into traditional CBA is currently widely considered to be a problematic task (Bauman et al., 2007; Cavill et al., 2008; Litman, 2013a; MacMillen, Givoni, \& Banister, 2010; 
Saelensminde, 2004; SQW, 2007). Wilson \& Cope (2011) also suggest that a journey ambience benefit should be counted for walkers and cyclists in CBA under the assumption that AT interventions often improve the quality of walkers' and cyclists' journeys.

\subsection{A review of cost-benefit analyses of active travel interventions}

In 2004, Saelensminde undertook a CBA on the AT investments of three Norwegian cities' (Trondheim, Hokksund \& Hamar). The CBA included both past and planned AT infrastructure in the three cities. The following benefits were included in the CBA: reduced insecurity (improved perceptions of safety for current \& new walkers/cyclists), reduced costs of transporting school children, reduced cost of work absenteeism, reduced cost of mortality, reduction of external costs road transport (local \& $\mathrm{CO}_{2}$ emissions, noise, congestion \& infrastructure costs) and reduced parking costs (Figure 2.1). The CBA assumed that travel time and accident rates do not increase as a result of the AT intervention. BCRs varied between the three cities. The BCR for Trondheim, the largest city, was estimated at 2.94. Hokksund and Hamar were estimated to give BCRs of 4.09 and 14.4 respectively. The CBA used a 25 year evaluation period and a $5 \%$ discount rate.

In 2009, Sloman, Cavill, Cope, Muller, \& Kennedy undertook a CBA on the Six Cycling Demonstration Towns project in the UK. The intervention included infrastructure, education and promotion components. The CBA on the Six Cycling Demonstration Towns project included, on the benefit side, only mortality savings based on the WHO's HEAT model. This intervention achieved significant results, with every age group reporting higher levels of cycling compared to baseline levels with the exception of those over the age of 75 (Figure 11). This CBA calculated a BCR of 2.59 using an evaluation period of 10 years and a discount rate of $3.5 \%$. The authors assume that a comprehensive analysis of benefits would have resulted in a much higher BCR than 2.59. 


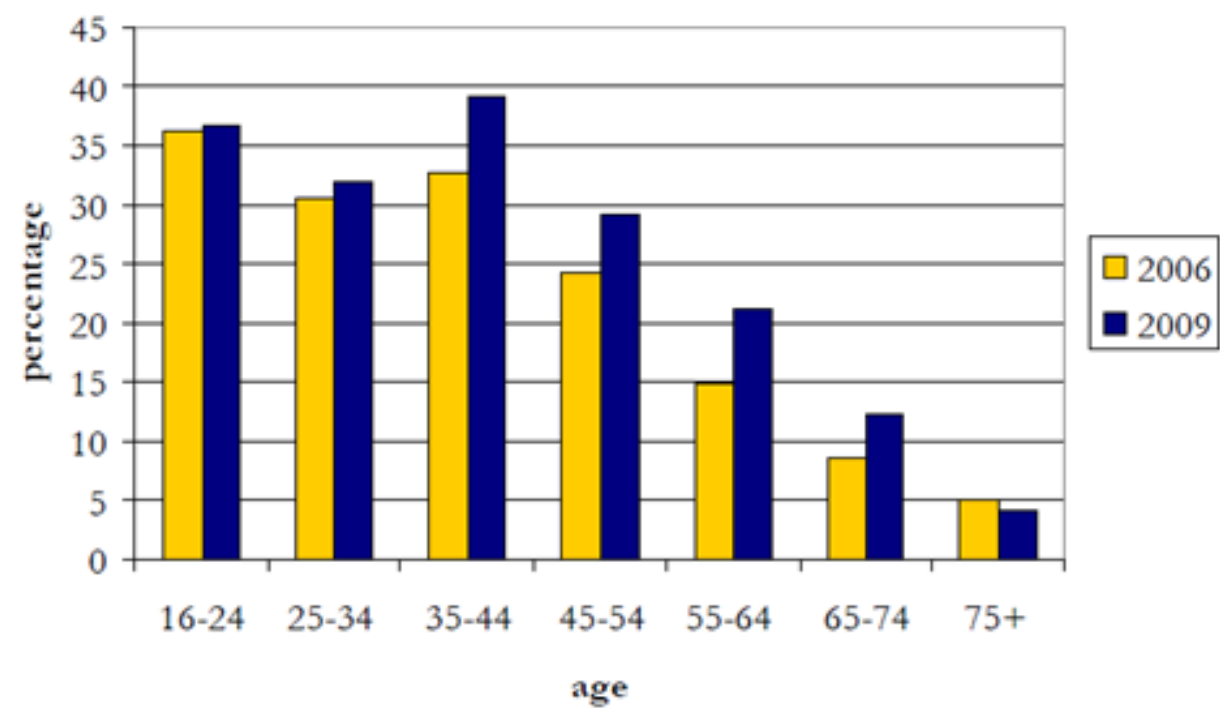

Figure 11: Proportion reporting any cycling in a typical week in the previous year, by age, from Sloman et al. (2009, p.12)

In 2011, Götschi undertook a CBA for past and estimated future AT investments in Portland, Oregon. Three planned investments were reviewed including a "Basic" plan, an $80 \%$ plan aiming to put $80 \%$ of Portland's population within $1 / 4$ mile of "low-stress bikeways", and a "World Class" option. BCRs were calculated for each plan with one model based on health care and fuel savings, and another model using mortality savings. BCRs for health care \& fuel savings models varied from 1.3 for the World Class option to 3.8 for the Basic option. Mortality savings alone achieved BCRs of 20.2 for the World Class option to 53.3 for the Basic option (Table 4). This CBA used a 40 year evaluation period at a $3 \%$ discount rate.

\begin{tabular}{lccc}
\hline & Baslc & $80 \%$ & World Class \\
\hline Investment costs (after discounting; incl. past) & $\$ 138$ & $\$ 296$ & $\$ 605$ \\
Projected mode share by 2030 & $15 \%$ & $20 \%$ & $25 \%$ \\
Max. annual bike miles (2030-2040) & $86 \mathrm{M}$ & $116 \mathrm{M}$ & $145 \mathrm{M}$ \\
Max. daily bike trips (3km trip length) & 60,000 & 80,000 & 100,000 \\
Cumulative bike miles 1991-2040 & $2200 \mathrm{M}$ & $2800 \mathrm{M}$ & $3400 \mathrm{M}$ \\
Cumulative health care savings 1991-2040 & $\$ 388$ & $\$ 491$ & $\$ 594$ \\
Cumulative fuel savings 1991-2040 & $\$ 143$ & $\$ 180$ & $\$ 218$ \\
Cumulative net benefits 1991-2040 & $\$ 394$ & $\$ 375$ & $\$ 207$ \\
Year to break even & 2015 & 2015 & 2032 \\
Annual lives saved (1991-2040 average) & 42 & 55 & 68 \\
Annual value of statistical lives saved (1991-2040 average) & $\$ 147$ & $\$ 196$ & $\$ 245$ \\
Cumulative value of statistical lives saved (1991-2040) & $\$ 7350$ & $\$ 9800$ & $\$ 12.250$ \\
Benefit-cost ratio for health care + fuel savings & 3.8 & 2.3 & 1.3 \\
Benefit-cost ratio for value of statistical lives saved & 53.3 & 33.1 & 20.2
\end{tabular}

Table 4: Key figures and results for 3 investment plans for bicycling in Porltand, from Götschi (2008, p.S54) 
In New Zealand, Macmillan (2012) performed a CBA estimating the effects of various scenarios for future AT investment in Auckland. Five scenarios were evaluated. The first scenario assumes business as usual. The second scenario is an AT investment similar to that proposed by the 2010 Auckland Regional Land Transport Strategy. The third scenario proposes creating separated cycle lanes on every arterial road in the region. The fourth scenario involves building a self-explaining road network, where local roads are constructed in a way that limits vehicle speeds and discourages through traffic, thereby encouraging higher pedestrian/cycling usage. The fifth scenario is a combination of the third and fourth scenarios, in effect a strategy that attempts to maximise the attractiveness of non-motorised means of transportation.

Macmillan's CBA includes commonly calculated benefits such as mortality, greenhouse gas and fuel savings. In addition to this, it includes a wide array of savings directly from conditions associated with air pollution based on a 'Health and Pollution in New Zealand' study that links and quantifies air pollutants' (including motor vehicle emissions') social costs including hospitalisations, mortality, Chronic Obstructive Pulmonary Disease (COPD) and restricted activity days. It is also worth noting that Macmillan assumes additional injuries and fatalities will occur as a result of these potential AT interventions in Auckland; these are calculated as costs to the interventions, an assumption not made in the other CBAs within this literature review. Scenarios three and four achieve the lowest BCRs at 11 and 6 respectively. Scenario two achieves a BCR of 18. Scenario five achieves the highest BCR at 22. This CBA used a 40 year evaluation period but presents non-discounted values. Mortality savings were large for all four scenarios, but were significantly exceeded by savings from restricted activity days as a result of a decrease in air pollutants. This author is unaware of other CBAs that have used restricted activity days as a benefit, nor of any CBAs that have presented undiscounted benefits. This economic evaluation was heavily reliant on transport modal shifts without the use of a robust traffic model for Auckland and also makes several assumptions on infrastructure costs, all of which adds some level of uncertainty to study results. The results are summarised in Table 5. 


\begin{tabular}{|c|c|c|c|c|}
\hline Outcome & $\begin{array}{l}\text { Scenario } 2 \\
\text { (monetised) }\end{array}$ & $\begin{array}{l}\text { Scenario 3 } \\
\text { (monetised) }\end{array}$ & $\begin{array}{l}\text { Scenario 4 } \\
\text { (monetised) }\end{array}$ & $\begin{array}{l}\text { Scenario } 5 \\
\text { (monetised) }\end{array}$ \\
\hline Cycling mode share by 2051 & $5 \%$ & $20 \%$ & $5 \%$ & $40 \%$ \\
\hline LV mode share by 2051 & $75 \%$ & $65 \%$ & $55 \%$ & $40 \%$ \\
\hline $\begin{array}{l}\text { Proportion of people } \\
\text { considering cycling } \\
\text { always/mostly safe by } 2040\end{array}$ & 0.4 & 0.69 & 0.3 & 0.9 \\
\hline LVKT (billion km) & -3.5 & -7 & -10 & -18.5 \\
\hline \multicolumn{5}{|l|}{ Extra cyclist injuries } \\
\hline $\begin{array}{l}\text { Fatal } \\
\text { Serious }\end{array}$ & $\begin{array}{l}200(620) \\
4000(1300)\end{array}$ & $\begin{array}{l}550(1700) \\
10400(3400)\end{array}$ & $\begin{array}{l}100(300) \\
2000(700)\end{array}$ & $\begin{array}{l}400(1200) \\
7700(2500)\end{array}$ \\
\hline $\begin{array}{l}\text { Air pollution } \\
\text { Mortality } \\
\text { Hospitalisations } \\
\text { COPD incidence } \\
\text { Restricted activity days } \\
\text { Air pollution total }\end{array}$ & $\begin{array}{l}-10(-7.5) \\
-5(-0.02) \\
-10(-0.75) \\
-12500(-1) \\
(-9)\end{array}$ & $\begin{array}{l}-20(-15) \\
-15(-0.04) \\
-30(-2.25) \\
-37200(-4) \\
(-21)\end{array}$ & $\begin{array}{l}-40(-30) \\
-20(-0.06) \\
-55(-4) \\
-57700(-6) \\
(-40)\end{array}$ & $\begin{array}{l}-80(-60) \\
-40(-0.12) \\
-90(-6.75) \\
-112200(-11) \\
(-78)\end{array}$ \\
\hline All-cause mortality & $-650(-2000)$ & $-1850(-5700)$ & $-650(-2000)$ & $-4000(-12400)$ \\
\hline $\begin{array}{l}\text { Greenhouse gas emissions } \\
\text { Megatons }\end{array}$ & $-3(-120)$ & $-8(-360)$ & $-13(-520)$ & $-26(-1040)$ \\
\hline $\begin{array}{l}\text { Fuel cost } \\
\text { million NZD }\end{array}$ & $(-600)$ & $(-1800)$ & $(-600)$ & $(-3900)$ \\
\hline Infrastructure cost & $(45)$ & $(250)$ & (380) & $(630)$ \\
\hline Net benefit (million NZD) & -770 & -2550 & -1780 & -13090 \\
\hline Benefit-Cost Ratio & 18 & 11 & 6 & 22 \\
\hline
\end{tabular}

Table 5: Summary of costs and benefits for scenarios 2-5, figures with a negative sign are savings, monetised figures are given in parentheses and all monetised figures are in millions of $\mathrm{NZ}$ dollars, from Macmillan (2012, p.262) 


\subsection{New Zealand Transport Agency's 2013 Economic Evaluation Manual cost-benefit analysis framework for active travel evaluation}

NZTA's 2013 EEM specifies that its CBA frameworks are designed to evaluate the economic efficiencies of proposed transport projects, including AT interventions. For interventions aimed at increasing cycling rates, NZTA provides a basic template for estimating "cycle demand" which uses formulas taking into account factors such as distance from infrastructure, population and population density, census cycle commute share in a base census year (2006) by city/district and relative benefit values based on type of AT improvement. NZTA recognises that successful walking and cycling interventions not only need improved facilities, they must also "connect appropriate origins and destinations, and use of the facilities must be promoted to encourage walking and cycling as alternative commuting modes" (NZTA, 2013, p.499). The EEM specifically states that its framework is not designed for post hoc economic evaluation, but for ex ante appraisal. For AT economic evaluation, the 2013 EEM stipulates the evaluation timeframe should be set at 40 years at a $6 \%$ discount rate.

\begin{tabular}{|c|c|c|c|c|c|c|c|}
\hline Cost & $\begin{array}{l}\text { चूँ } \\
\text { ळ }\end{array}$ & 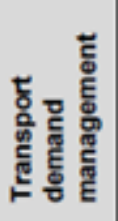 & 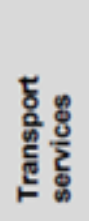 & 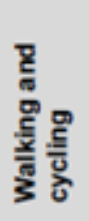 & 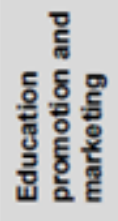 & 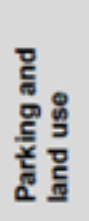 & 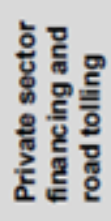 \\
\hline Construction cost & $\checkmark$ & $\checkmark$ & $\checkmark$ & $\checkmark$ & $\checkmark$ & & \\
\hline Maintenance costs & $\checkmark$ & $\checkmark$ & $\checkmark$ & $\checkmark$ & $\checkmark$ & & \\
\hline Operating cost & $\checkmark$ & $\checkmark$ & $\checkmark$ & $\checkmark$ & $\checkmark$ & $\checkmark$ & $\checkmark$ \\
\hline Funding gap & $\checkmark$ & $\checkmark$ & & $\checkmark$ & 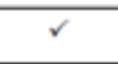 & 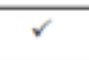 & \\
\hline Nett land cost & $\checkmark$ & & $r$ & $\checkmark$ & & $\checkmark$ & \\
\hline Decommissioning / revocation costs & $\checkmark$ & & $\checkmark$ & & & & \\
\hline Planning and design costs & $\checkmark$ & $\checkmark$ & $\checkmark$ & $\checkmark$ & $\checkmark$ & $\checkmark$ & $\checkmark$ \\
\hline Finance costs & & & & & & & $\checkmark$ \\
\hline
\end{tabular}

Table 6: NZTA's EEM list of costs by facility type, from NZTA (2013, p.2-13) 
In addition to the costs defined by NZTA for AT interventions that have already been mentioned (Table 6), there are a few additional costs that are relevant to economic evaluation of AT interventions. The 2013 EEM places different values on time depending on mode of transportation and travel purpose. Although cars' occupants are assigned a slightly higher value of time than walkers or cyclists (NZ\$ 23.85 vs. NZ\$ 21.70

\begin{tabular}{|l|c|c|c|}
\hline Vehicle occupant & $\begin{array}{c}\text { Work travel } \\
\text { purpose }\end{array}$ & $\begin{array}{c}\text { Commuting } \\
\text { to/from work }\end{array}$ & $\begin{array}{c}\text { Other non-work } \\
\text { travel purposes }\end{array}$ \\
\hline \multicolumn{3}{|l|}{} & \multicolumn{2}{|l|}{} \\
\hline Base values of time for uncongested traffic $\mathbf{( \$ / h )}$ & 23.85 & 7.80 & 6.90 \\
\hline Car, motorcycle passenger & 21.70 & 5.85 & 5.20 \\
\hline Light commercial driver & 23.45 & 7.80 & 6.90 \\
\hline Light commercial passenger & 21.70 & 5.85 & 5.20 \\
\hline Medium/heavy commercial driver & 20.10 & 7.80 & 6.90 \\
\hline Medium/heavy commercial passenger & 20.10 & 5.85 & 5.20 \\
\hline Seated bus and train passenger & 21.70 & 4.70 & 3.05 \\
\hline Standing bus and train passenger & 21.70 & 6.60 & 4.25 \\
\hline Pedestrian and cyclist & 21.70 & 6.60 & 4.25 \\
\hline
\end{tabular}

Table 7: EEM behavioural values of time for vehicle occupants, from NZTA (2013, p.5-204)

for commuting to work purposes, Table 7), the switch from driving to walking/cycling arising from an intervention is said to be recognised in benefits assigned to walking/cycling such as health gains and road traffic reduction. The 2013 EEM stipulates that travel time savings can be a benefit for walking or cycling if the intervention improves their or other drivers' commute time, or potentially a cost if the intervention creates longer travel times for other users, such as car drivers. The 2013 EEM allows for interventions that promote both walking and cycling to claim both benefits. However, if the promotion of walking or cycling is expected to create conflict between walkers and cyclists (due to limited space), then the evaluation must account for this. A recent study by Börjesson \& Eliasson (2012) found that the value of time for cyclists should be higher than alternative modes on the basis that cycling itself is perceived as being “onerous”; thus faster routes can reduce this sense of burden. This argument is challenged by Litman (2013c), who suggest that travel time costs and savings are largely dependent on the context they are presented in; the cost of time for a cyclist riding for recreation purposes in quiet environment would be very low in comparison to a cyclist commuting to work on a congested urban arterial. 
One method of calculating benefits from an AT intervention is by using composite values provided by NZTA which account for benefits such as health, road traffic reduction and safety (shown in the Tables 8 and 9). These values can be applied to new $\mathrm{km}$ walked/cycled using a new facility. The basis of these values is not explained within the 2013 EEM document itself, and may rest on some contestable assumptions.

\begin{tabular}{|l|l|}
\hline Benefit & Benefit per pedestrian $(\mathrm{km})$ \\
\hline Health & 2.60 \\
\hline Safety & 0.00 \\
\hline Road traffic reduction & 0.10 \\
\hline Composite benefit & 2.70 \\
\hline
\end{tabular}

Table 8: EEM composite benefits for walkers in NZ\$, from NZTA (2013, p.5-504)

\begin{tabular}{|l|l|}
\hline Benefit & Benefit per cyclist $(\mathbf{k m})$ \\
\hline Health & 1.30 \\
\hline Safety & 0.05 \\
\hline Road traffic reduction & 0.10 \\
\hline Composite benefit & 1.45 \\
\hline
\end{tabular}

Table 9: EEM composite benefits for cyclists in NZ\$, from NZTA (2013, p.5-504)

In addition to the safety benefit stipulated in the above tables (no benefit for walkers, NZ $\$$ 0.05 per $\mathrm{km}$ for cyclists), the $2013 \mathrm{EEM}$ also allows for a per trip benefit of $\mathrm{NZ} \$ 2.70$ for walkers (Table 8 ) and NZ $\$ 4.35$ for cyclists (Table 9) where interventions remove impediments to safe walking and cycling. The 2013 EEM suggests using surveys and/or other methods of research to determine what users perceive as impediments to safe walking and cycling.

NZTA's 2013 EEM allows for other benefits to be considered in economic evaluation of AT interventions. Benefits other than health, safety and road traffic reduction may be included, but the 2013 EEM provides less guidance on how these benefits should be estimated for AT economic evaluation. The 2013 EEM provides an average yearly operating cost of NZ\$12,222 per private motor vehicle. Vehicle operator cost savings may be applied when a user either drives less or forgoes ownership of a motor vehicle as a result of an AT intervention. Crash cost savings may occur if an AT intervention is thought to reduce the overall number of vehicular accidents, although this could also be a 
cost if an AT intervention is thought to increase the number of crashes. The EEM does in principle recognise that improvements in AT infrastructure and corresponding modal shift from driving to walking/cycling can result in reduced crash rates per walker/cyclist and therefore suggests that in most cases AT interventions should not incur any crash costs, and in certain cases can be credited crash cost savings. There is considerable literature supporting this assumption under the theory of "safety in numbers" which suggests that as numbers of cyclists/walkers increase, cyclist/walker crash rates decrease (Genter \& NZ Transport Agency, 2009; Jacobsen, 2003; Litman, 2013a; SQW, 2007; Tin, Woodward, Thornley, \& Ameratunga, 2011).

The current EEM does not ascribe explicit vehicle emission benefits; instead these are said to be accounted for within the road traffic reduction benefit that forms part of the composite value assigned per AT trip, but the latter is not explicitly explained, as noted above. The 2013 EEM recognises that AT interventions can provide external benefits, mostly from reduced road usage and hence reduced need for motor vehicle infrastructure. These benefits can include reduction in traffic noise, improved visual impacts and reduced community severance. As is the case with most of the literature available for AT economic evaluation, the 2013 EEM provides little concrete guidance on how to apply these benefits to economic evaluation of AT interventions. The 2013 EEM also lists journey time reliability as a potential benefit of AT interventions under the assumption that AT users are less constrained by motor vehicle congestion and availability of public transport; however no value for this is given, probably because it is difficult to quantify or monetise in any meaningful way. However, the road traffic reduction benefit component of the walking and cycling composite value may account for this.

As the 2013 EEM CBA framework is designed to appraise proposed projects as opposed to evaluating a completed one, appraisal of AT interventions is heavily reliant on AT demand analysis. The 2013

EEM provides guidance on predicting demand for cycling facilities. This cycling demand analysis takes into account relative benefit of various infrastructure types (Table 10). The relative benefit represents

\begin{tabular}{|l|l|} 
Type of cycle facility & Relative benefit \\
\hline On-street with parking (no marked cycle lane) & 1.0 \\
\hline On-street with parking (marked cycle lane) & 1.8 \\
\hline On-street without parking (marked cycle lane) & 1.8 \\
\hline Off-street cycle path & 2.0 \\
\hline
\end{tabular}

Table 10: EEM relative benefits for different types of cycle facilities, from NZTA (2013, 5-502) 
the amount of time a cyclist would spend travelling in each facility relative to the baseline facility (e.g. on-street lane with parking, but no marked cycle lane), based on a previously conducted stated preference survey. According to these findings, a cyclist will gain twice the benefit on an off-street cycle path as they would on the baseline facility. However, in comparison to a facility that is on-street with a marked cycle lane with no parking, the cyclist would only gain $5.3 \%$ more benefit on the off-street cycle path.

\subsection{Critiquing past active travel economic evaluation practice}

Much of the AT of literature critiques past AT interventions, or intervention studies, often with recommendations on how the interventions or intervention studies can be improved.

Pucher, Dill, \& Handy (2010) argue that AT interventions sometimes focus too heavily upon improving segments of infrastructure instead of building networks. Pucher et al. also suggest that, because of synergies, the value of a network is thought to be beyond the sum of the values of the individual segments. Frank \& Engelke (2001) stress that an individual's ability to reduce their reliance on motor vehicle transport requires the availability of competitive forms of transport, including reliable public transport and AT infrastructure that allows users to reach their destinations with minimal levels of inconvenience and discomfort.

Some literature suggests that past AT interventions have focussed too heavily on infrastructure improvements and suggests that AT interventions should package together infrastructure and promotional/educational components to maximise modal shift to walking and cycling (Banister \& Gallent, 1999; Pucher et al., 2010; SQW, 2007). Ogilvie, Egan, Hamilton, \& Petticrew (2004) argue that education/promotion needs to be a participatory process where individuals'/groups' specific needs are addressed. Ogilvie, Mitchell, Mutrie, Petticrew, \& Platt (2010) stress that interventions need to take into account the needs of low-income and minority neighbourhoods, as they may not share transport policy-makers' enthusiasm for improved AT infrastructure. A recent study by Goodman, Sahlqvist, \& Ogilvie (2013) found that new users of an intervention that improved walking and cycling facilities in three UK municipalities were disproportionately used by physically fit and socio-economically advantaged users. Issues such as crime are 
found to be a deterrent to AT participation, especially within communities of high deprivation (Joh, Nguyen, \& Boarnet, 2011; Ogilvie et al., 2010).

Some literature suggests that past AT interventions have focussed too much on on-street improvements, ignoring what is likely a significant portion of the population who are unwilling to cycle in close-proximity to motor vehicle traffic (Forsyth \& Krizek, 2010; Hunt \& Abraham, 2006; Kingham, Taylor, Koorey, \& NZ Transport Agency, 2011; Ogilvie et al., 2010; Pucher \& Buehler, 2008; Yeates, 2001). There are differing opinions on whether or not separated bicycle facilities actually improve safety of cyclists. Jacobsen (2003) argues that separated bicycle facilities can result in lower accident rates for cyclists while Forsyth \& Krizek (2010) suggest that separated bicycle facilities may not reduce a cyclist's actual risk of accident; however, separated bicycle facilities tend to improve cyclists' perception of safety. Some research claims that perception of safety is more important to a cyclist than actual risk reduction (Garrard, Rose, \& Lo, 2008; McClintock, 2002), while other research suggests that perception of safety is the most important factor for whether or not an individual chooses to cycle (Daley, Rissel, \& Lloyd, 2007; Rietveld, 2000; Winters, Davidson, Kao, \& Teschke, 2010). Ogilvie et al. (2010) argue that young and inexperienced cyclists in particular are reliant on separated bicycle facilities in order to become regular cyclists. In the New Zealand context, Muggeridge (2012) found that in the provincial city of Hastings cycling (prior to the MCP) is not perceived as a safe activity and interventions should create new separated bicycle facilities to enhance the perception of cycling as a safe travel option.

\subsection{Critique of cost-benefit analysis as an evaluation tool for active travel interventions}

There is a wide array of literature that critiques contemporary economic evaluation of AT interventions.

Calculating benefits from AT interventions is a problematic process (Saelensminde, 2004; SQW, 2007; Wilson \& Cope, 2011). Contemporary CBA does not fully account for all the potential benefits of AT interventions (Litman, 1998; Ogilvie et al., 2004; SQW, 2007). In order to monetise or even quantify benefits, an evaluator is required to make assumptions - if these are explicit, an evaluator's bias is revealed (Damart \& Roy, 2009; Lawrence D. Frank, 2004; Genter \& NZ Transport Agency, 2009; Litman, 2013a). Cavill et al. (2008) argue that AT CBA tends to focus on mortality savings while few studies even 
attempt to value morbidity savings. A few authors argue that methods of assessing improvements in morbidity are needed to fully account for health savings due to an AT intervention (UK Department for Transport, 2012; Wilson \& Cope, 2011), although this author is unaware of any available robust measure of morbidity. Börjesson and Eliasson (2012) argue that cycling demand is more closely linked to time savings and infrastructure quality; thus CBA should value these higher than health savings. Economic evaluation of AT interventions rarely incorporates benefits to children as their relative health risk for death is comparatively low in comparison to adults (SQW, 2007).

Economic evaluation of AT interventions relies on data representing modal shift to walking and cycling as a result of an intervention. Accurate collection of these data is notoriously difficult and often reliant on a small window of analysis potentially leading to error in data collection and estimation (Damart \& Roy, 2009; Ogilvie, 2004; Ogilvie et al., 2011; Rietveld, 2000; Saelens, Sallis, \& Frank, 2003). Saelens et al. (2003) also suggest that usage of self-reported data from user surveys can add more potential error, although such data are widely used for a range of transport users. In terms of post-hoc evaluations, Blanco et al. (2009) report that most evaluations have not used robust research designs using baseline data as well as treatment and control groups. Pucher et al. (2010) are concerned that past evaluations have often failed to determine causality, or whether or not increases in modal share are the direct result of the AT investment. Cavill et al. (2008) argue that past AT studies also fail to account for individuals who replace another activity with walking or cycling. Ogilvie (2004) also found that evaluations often do not account for cycling increases by existing cyclists, as this is generally less beneficial than a new cyclist taking up cycling. Ogilvie (2004) also identifies a potential weakness of AT evaluations as that they are incapable of accounting for existing walkers who may shift to cycling as a result of an AT intervention.

\section{Authors such as Bateman, Turner, \& Bateman (2005), Boardman, Moore, \& Vining} (2010) and Damart \& Roy (2009) suggest that high discount rates can distort conclusions: if an intervention is rejected, due to future benefits being highly discounted, it potentially robs future generations of long-lived interventions that could improve their quality of life. Boardman et al. suggest adopting "social discounting” for projects whose effects "occur over decades or more" and for "others that seek to avert climate change, [and] could have impacts that span centuries" (2010, p.326). Damart \& Roy (2009) argue that CBA often provides very low benefit values for the future, and suggests social discount rates should be set at $4 \%$ for interventions that account for equity and other intergenerational 
considerations. In the 2006 UK Government-sponsored report entitled "Stern Review on the Economics of Climate Change", lead author Nicholas Stern recommends adopting a discount rate of $1.4 \%$ in economic evaluation of projects that help mitigate climate change (Stern, 2006). Dietz, Anderson, Stern, Taylor, \& Zenghelis further highlight the future potential catastrophic costs of climate change from business as usual; thus discount rates must reflect risk and ethical considerations for future generations who will bear greater costs from previous generations' greenhouse gas emissions (2007). Other authors such as Pettit (2013) and Boardman et al. (2010) recommend adoption of diminishing discount rates over time to better account for these intergenerational considerations.

In the UK, the Department for Transport stipulates in their Transport Analysis Guidance of the Appraisal of Walking and Cycling Schemes that AT interventions should be evaluated at a discount rate of $3.5 \%$ for the first thirty years, and $3 \%$ for an additional thirty years (UK Department for Transport, 2012). These evaluative conditions allow for a diminishing discount rate as well as a longer evaluation time period. Pettit (2013) notes that diminishing discount rate schemes are currently employed by transport authorities in the UK and France, and proposes Social and Composite Discount Rates for evaluation of transport projects in New Zealand (Table 11). The social discount rate is meant to be applied for projects that are expected to improve conditions of equity and accessibility for vulnerable population sub-groups.

\begin{tabular}{lllll}
\hline Year of Project & UK & France & NZ Social & NZ Compromise \\
\hline $0-30$ & $5.5 \%$ & $4 \%$ & $5 \%$ & $4 \%$ \\
$31-75$ & $3 \%$ & $2 \%$ & $2 \%$ & $2.5 \%$ \\
$76-125$ & $2.5 \%$ & $2 \%$ & $1.5 \%$ & $2.25 \%$ \\
$126-200$ & $2 \%$ & $2 \%$ & $1 \%$ & $2 \%$ \\
$201-300$ & $1.5 \%$ & $2 \%$ & $1 \%$ & $1.5 \%$ \\
$501-$ & $1 \%$ & $2 \%$ & $1 \%$ & $1 \%$ \\
\hline
\end{tabular}

Table 11: Existing international and proposed NZ discounting schemes, from Pettit (2013, p.201-11) 
Damart \& Roy (2009) suggests CBA has another complexity in that values of statistical lives vary widely across countries as well as between government agencies within the same country, and such values can even depend upon how an individual dies. Damart \& Roy (2009) also point out that CBA used in appraisal is designed to choose the best possible investments based on exhaustive and unbiased studies of project costs and benefits, and considering multiple options, although the author concludes that this aspiration is unrealistic.

Other authors argue that CBA frameworks are typically biased towards motor vehicle transport. Veisten et al. (2007), Wilson \& Cope (2011), and Cervero (2011) suggest that many frameworks are too reliant on travel time savings, which make motor vehicle infrastructure more attractive at the expense of non-motorised means of transport. Damart \& Roy (2009), Genter \& NZ Transport Agency (2009), Pettit (2013), Litman (2013c), and Saelensminde (2003) argue that travel time savings values should be linked to the context they are presented in, implying that motor vehicle users should have different time travel values than non-motor vehicle users. CBA tends to underestimate external costs of motor vehicle transport and encourages "predict and provide" transportation policies, which tend to increase motor vehicle dependency in urban areas at the expense of other alternative modes of transport (Banister, Turner, Richardson, \& Rees Jeffreys Road Fund, 2000; Cervero, 2011; Dora et al., 2000; Lawrence D. Frank, 2004; Litman, 2006, 2013a; MacMillen et al., 2010; Ruta, 2002).

\subsection{Alternative methods of evaluating active travel interventions}

Mees \& Dodson (2006) argue that planning processes and competing interests have thus far prevented meaningful transport investments in the Auckland context that promote well-being, sustainability and reduce car dependency. They cite lack of transparency and poor community consultation as primary factors why Auckland has invested so heavily in automobile infrastructure at the expense of alternative modes. Macmillan (2012) cites overseas implementation of Health Impact Assessments (HIAs) as an effective means of balancing evaluation of transport infrastructure in order to better account for external costs of motor vehicle infrastructure and the benefits of infrastructure promoting AT. Macmillan points out that HIA has already been used in the NZ context, but often too late within evaluation/decision-making processes. 
MacMillen et al. (2010) argue that qualitative evaluations such as Multi-Criteria Analysis (MCA) should complement traditional methods of economic analysis to provide more robust AT evaluation frameworks. In simple terms, this form of qualitative analysis allows evaluators to account for benefits that are difficult to monetise or quantify in any meaningful way (2010) as well as providing a more transparent approach to equity (Shiftan \& Shefer, 2009; Vreeker, Nijkamp, \& Ter Welle, 2002). In 1998, in recognition of the UK society's increasing dependence on motor vehicles and the effect this dependence was having on the surrounding environment and global climate change, the UK Department for Transport adopted a new transport policy entitled “A New Deal Transport: Better for Everyone” (UK Department for Transport, 2014). Within this, a new transport evaluation framework called the New Approach to Appraisal (NATA) was adopted (Macmillen et al., 2010).

NATA in simple terms requires MCA analysis to complement traditional CBA, and changed CBA's role from a decision-making tool to simply another factor in transport decision-making (Macmillen et al., 2010). Despite this diminished role, CBA in the UK is still considered the most useful component to evaluation of proposed transport projects as it allows for easy - if perhaps misleading - comparison between projects (Glaister, 1999). Preston (2009), Bateman et al. (2010) and Boyce (2010) agree that the MCA and qualitative-based methods of evaluation are more holistic and effective at evaluating multiple social goals, especially in relation to issues of sustainability, equality, social inclusion and other factors related to quality of life.

Some authors question the need for economic evaluation of AT interventions at all. Genter \& NZ Transport Agency (2009) recognise that NZ and the UK are among the few countries that require economic evaluation for AT interventions to receive prioritised national funding. Nations such as Germany, Denmark and the Netherlands, which have higher modal share for walking and cycling compared to other developed countries, do not require economic evaluation of AT interventions in order to be eligible for national funding schemes (Genter \& NZ Transport Agency, 2009; Macmillen et al., 2010). A report by van Goeverden \& Godefrooij (2011) suggests that large scale AT investments in the Netherlands were implemented in the 1970s in order to promote alternative modes of transport to counteract the increasingly apparent negative impacts of automobile use in urban areas. The United Nations Environment Programme (2010) suggests that successful walking and cycling interventions in Denmark and the Netherlands are a result 
of concerted and comprehensive efforts to invest in AT facilities in the face of diminishing rates of both walking and cycling and increasing costs of oil.

These facts may suggest that if higher AT modal shift is a primary objective of contemporary transport and related policy, such as health or urban policy, reliance on conventional CBA-based economic evaluation may be undesirable. Studies commonly show a strong correlation between AT investment and AT modal share as seen in a United Nations Environment Programme (2010) report entitled "Share the Road" (Table 12). A recent report by Craig (2013) on behalf of the Canadian Clean Air Partnership estimates 20 million Canadian Dollars can widen a two lane road to four lanes for $8 \mathrm{~km}$. In contrast, this 20 million dollars could also build approximately $260 \mathrm{~km}$ of separated cycled tracks, or 1,000 km of on-road cycle lanes (Figure 12). These figures highlight the significant cost disparities between motor vehicle and active travel facilities.

\begin{tabular}{l|l|l} 
& \multicolumn{1}{|c}{$\begin{array}{c}\text { Annual Funding } \\
\text { (USD per resident) }\end{array}$} & Cycling Mode share \\
\hline USA & 1.5 & $1 \%$ \\
\hline Portland (City in Western US) & 3.5 & $4 \%$ \\
\hline Berlin & 6 & $10 \%$ \\
\hline Copenhagen & 13 & $20 \%$ \\
\hline Amsterdam & 39 & $35 \%$
\end{tabular}

Table 12: Correlation between cycling infrastructure funding and cycling overall mode share, from UNEP (2010, p.25)

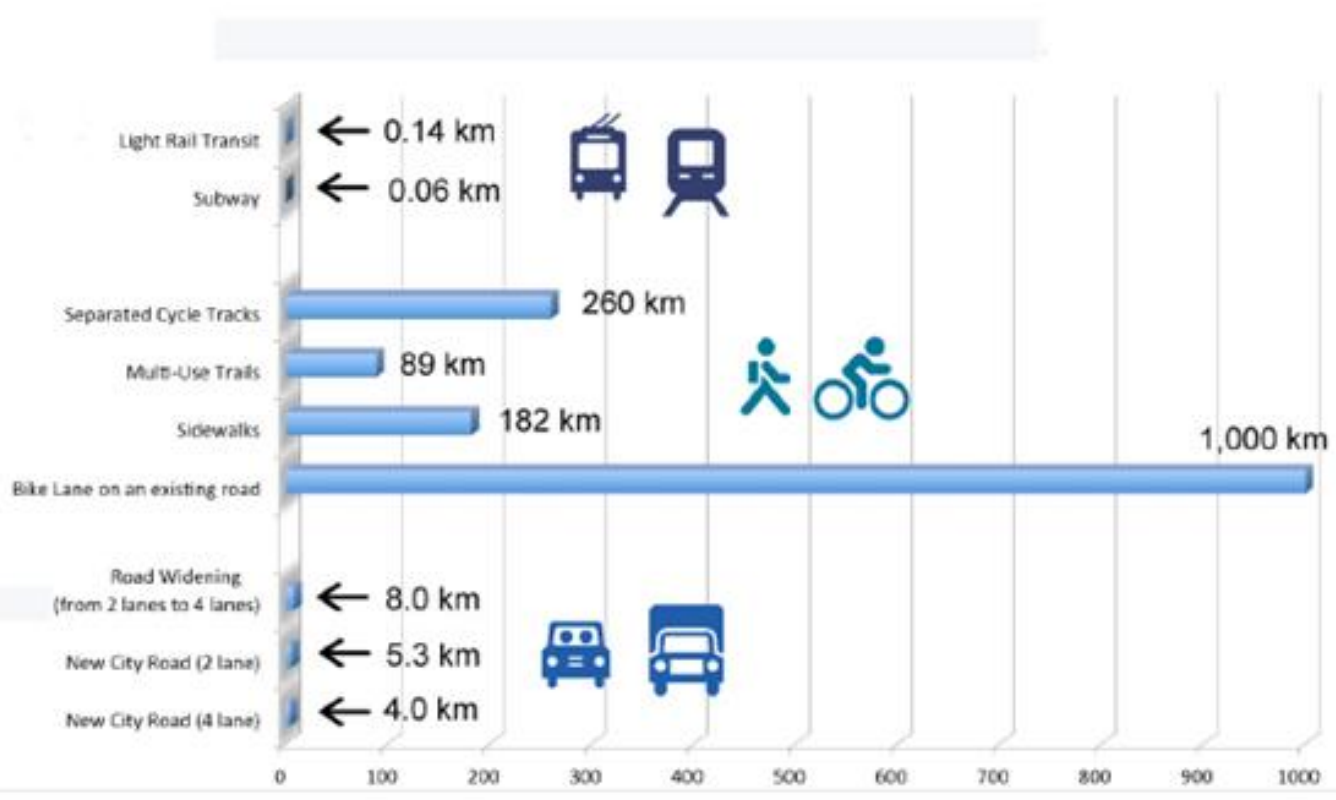

Figure 12: How many kilometres could be built for \$20 million, per km costs, from Craig (2013, p.13) 


\section{Chapter 4 - Aims and approach}

The previous chapter set out to review the literature on economic evaluation of investment for interventions promoting active travel. The literature has highlighted several weaknesses in evaluation of these interventions including: an overreliance on quantified and monetised benefits at the expense of those benefits that cannot be quantified or monetised, faulty research designs and the use in some evaluations of narrow research scopes. It also pointed to a significant gap in the literature relating to the thorough evaluation of walking and cycling interventions in the New Zealand context. Theories linking investment in active travel in the New Zealand context to higher rates of walking and cycling are to date largely based on assumptions rather than objective and rigorous analysis, as evidenced by the lack of post hoc evaluations of walking and cycling interventions in New Zealand. These subjective assumptions are not necessarily erroneous; however they represent untested hypotheses.

In light of this, the present study sets out to conduct an evaluation of the effectiveness of national and local government spending on walking and cycling interventions to boost participation rates for active modes of travel. The primary aim of this research is to address the following question:

\section{Is expenditure on walking and cycling interventions of a significant scale worthwhile within the urban New Zealand context?}

To answer the above question, this study provides an evaluation of the Model Communities Programme, a targeted, medium-scale walking and cycling intervention spearheaded by NZTA to boost walking and cycling rates in Hastings and New Plymouth. The study breaks the evaluation into three parts. After a methodological discussion in Chapter 5, Chapter 6 undertakes a conventional CBA in order to determine the perceived economic efficiency of the Model Communities Programme. Chapter 7 carries out a mixed methods extended analysis of the MCP users' perceptions of benefits from the intervention. Chapter 8 provides an evaluation of the successes and weaknesses of the MCP intervention based on qualitative data collected from interviews with project managers in Hastings and New Plymouth. Chapter 9 pulls these threads together in an overall discussion. 


\section{Chapter 5 - Methodology}

\subsection{Methodological approach}

This study employs a pragmatic approach. J. Creswell \& Plano Clark (2011) underline the pragmatic approach's ability to focus on the research problem and the questions that surround it. In relation to this study's evaluation of the MCP, the base problem is low rates of walking and cycling in New Zealand while the primary research question is investigating whether or not national investment in active travel interventions is likely to boost walking and cycling rates. A pragmatic approach allows for a broadened focus and an opportunity to prioritise key issues such as health, safety, behaviour and other issues that are perceived as receiving inadequate levels of public and/or institutional attention (Glasgow, 2013). Glasgow states that the overall goal of a pragmatic approach is "to produce results that are relevant to stakeholders as well as rigorous”, while stressing a "focus on application and context" (2013, p.257). There are a number of variables that affect active travel participation rates, and while the level of funding of active travel infrastructure is often regarded as one of the most important influential factors, analysis of previous studies have shown that this is not necessarily true (Cavill et al., 2008).

In chapter 4, we noted the lack of careful testing of propositions about active travel investment in New Zealand. In appraising the MCP, a pragmatic approach allows for objective and subjective analysis of theories about active travel (J. W. Creswell, Klassen, Plano Clark, \& Smith, 2011), in this case analysing the relationship between national investment in active travel interventions and its impact on active travel participation rates.

A mixed methods strategy has been chosen to explore this topic. The first method, set out in Chapter 6, is an economic evaluation in the form of four CBA simulations using quantified and monetised data for estimating costs and benefits of the Model Communities Programme. A second method is set out in Chapter 7, which undertakes an analysis of quantitative and qualitative data in order to evaluate several benefits that are difficult to monetise, and in some cases are difficult to even quantify, and thus do not fit within the conventional CBA frameworks. Chapter 8 provides a brief analysis of qualitative data provided by project managers in relation to their perceptions of intervention successes and weaknesses. The combination of these three research chapters will allow for triangulation of the research question (Guion, Diehl, \& McDonald, 2011), and more thorough evaluation of the MCP. 
This mixed methods strategy provides some key advantages for this study. Although the benefits of active travel are generally known, there is less agreement in terms of how these benefits should be quantified or monetised. Safety in particular is a contested benefit in active travel. Hopkinson \& Wardman (1996) and Saelensminde (2004) have strongly argued that safety is regularly undervalued in economic evaluation of walking and cycling. The fact that the 2013 NZTA EEM's “Composite Benefit” attributes a safety benefit of only NZ $\$ 0.05$ per $\mathrm{km}$ cycled and no value to $\mathrm{km}$ walked could suggest that safety for active users is undervalued by national transportation authorities. Some benefits, especially those in relation to improved community outcomes, are known but are difficult to quantify in any meaningful way. Using a mixed methods strategy allows for a more thorough analysis of both certain and less certain phenomena (J. W. Creswell et al., 2011), or in this study's case, benefits associated with active travel. Bryman (2008) further adds that mixed methods strategies may provide numerous advantages to this type of study including: a more complete answer to the study's research question, a research approach that better reflects the contextual conditions of the study, and an enhanced ability to gather data that reflect a diverse range of viewpoints or even allowing for the reception of unexpected results. Elliott underlines the merits an approach that uses both quantitative and qualitative methodologies, arguing that studies using "mixed methods can potentially capitalise on the strengths of each approach” (2005, p.172).

Victoria University of Wellington Human Ethics Committee granted final approval for this research on September $17^{\text {th }}$, 2013. A copy of this letter can be found in Appendix A.

\subsection{Cost-benefit analysis of the Model Communities Programme: analysis of costs}

Intervention costs were estimated using MCP data from district councils. These data were gathered during interviews with MCP project managers, and in some cases were taken directly from the council's NZTA MCP proposals. The costs included in this analysis are infrastructure costs, education/promotion costs and maintenance costs. Infrastructure costs include costs of construction for new/improved facilities built during the initial 2010-2012 MCP funding period, data which was provided by the project managers.

Education/promotion costs are calculated using two components: total education/promotion spending during the initial MCP funding period (provided by the 
project managers) and during the remaining years within the evaluation timeline were calculated at a per annum diminishing rate of $20 \%$ from the 2013 education/promotion spending. The latter calculation takes into account the project managers' desires to continue education/promotion spending for their programmes, but also reflects the uncertainties of future district council funding and the likelihood that this funding will diminish over time.

Annual maintenance costs were based on figures listed in each district council's MCP proposals, set at 2013 maintenance spending (NZ\$ 50,000 for Hastings, NZ\$134,838 for New Plymouth). Monitoring and evaluation costs are not included in this cost analysis as they are modest and unlikely to have any influence on user demand (although they may influence levels of funding for future spending on similar programmes). A more detailed disclosure of MCP cost estimates can be found in Appendix B.

\subsection{Estimating walking and cycling rates}

Estimating rates of walking and cycling as a result of an intervention is a difficult process that faces a multitude of uncertainties (Krizek et al., 2006). This process is typically reliant on analysis of a narrow range of data, potentially leading to a high risk of estimation error (Ogilvie et al., 2004; Rietveld, 2000; Saelens et al., 2003). There are a limited number of studies that undertake economic evaluation of interventions that attempted to create networks of walking/cycling infrastructure. Saelensminde's (2004) previously mentioned CBA simply assumes a rate of induced demand as a result of the improved walking and cycling infrastructure. Götschi (2011) used cycling count data from several bridges with separated bicycle facilities to estimate potential cycling growth rates.

MCP project managers have provided walker and cyclist counts from some of their key routes. Given the relatively expansive nature of the MCP infrastructure, these walker and cyclist' counts can only provide a limited window on walkers' and cyclists' overall continuing use of the infrastructure.

New Zealand Census Data provide another opportunity for analysing changes in walking and cycling rates, although its focus is solely on trips to work. However, Census data does have an advantage of relatively larger samples sizes in comparison to other data sets, and thus is less prone to sampling error. Krizek et al. (2006) argue that census travel to work data can be an accessible data set that can better capture regular active users than can to 
other types of data. Given the large benefits that are usually estimated for regular users in the form of health savings, it is important to make accurate estimations of the proportion of observed trips that can be attributed to regular users.

This study has reviewed Census data from four periods (1996, 2001, 2006 and 2013) to estimate changes in walking and cycling rates in Hastings and New Plymouth, the two councils receiving the intervention. To account for the potential of wider trends influencing active travel rates irrespective of the intervention, the walking and cycling rates were compared to national data as well as data from two control cities, Masterton and Wanganui.

The analysis of these two control cities mirrors a quasi-experimental design formulated by the authors of the ACTIVE study (Chapman et al., 2014). The aim of the ACTIVE study is to assess whether physical activity, walking and cycling habits, and attitudes and perceptions, change as a result of the MCP intervention. To achieve this, the ACTIVE study interviewed individuals from randomly selected households in the intervention cities (Hastings and New Plymouth) as well as the two control cities (Masterton and Wanganui). The present thesis contributes to the wider ACTIVE study by providing an economic analysis of the MCP. The present research also provides key stakeholder analysis from users of MCP infrastructure as well as feedback from project managers from both intervention cities.

Masterton and Wanganui were chosen as control cities (for the ACTIVE study) as they hold similar geographic and demographic attributes as the intervention cities, but were not given national funding for a walking and cycling intervention (Chapman et al., 2014). The use of these control cities allows for this evaluation to infer causality, confirming the likelihood that the intervention is the origin of the changing rates of walking and cycling. It is important to note that the Census data only account for walking and cycling trips to work, which ignores trips for other purposes. Weighted data from the New Zealand Household Travel Survey (NZHTS) provide figures for all trip purposes for walking and cycling; these data have been analysed to confirm trends observed by the Census data. 


\begin{tabular}{|lrrrrrr|}
\hline & \multicolumn{7}{c|}{ Walking } & \multicolumn{7}{c|}{ Cycling } \\
& \multicolumn{1}{c}{$1996-$} & $2001-$ & $2006-$ & $1996-$ & $2001-$ & $2006-$ \\
& \multicolumn{1}{c}{2001} & 2006 & 2013 & 2001 & 2006 & 2013 \\
\hline Intervention & -9.1 & 11.1 & -6.6 & -30.7 & -6.1 & 20.7 \\
Control & 0.2 & 2.7 & -17.7 & -22.7 & -25.5 & -13.5 \\
National & 0.1 & 14.0 & -6.9 & -23.3 & -19.2 & 13.1 \\
\hline
\end{tabular}

Table 13:\% changes in \# of walking and cycling trips to work (1996-2013 Census Data)

Census data from 2006 to 2013 show that overall walking rates are in decline across New Zealand. Data calculating the percentage change for the number of trips walked to work from 2006 to 2013 (Table 13) has declined for the intervention cities (-6.6\%) and nationally $(-6.9 \%)$, both at a significantly lower rate than in the control cities $(-17.7 \%)$.

Although one might be tempted to argue that the intervention rates are nearly the same as the national rates, weighted NZHTS data shows that a sample of large cities ${ }^{1}$ had percentage changes for all reported walking trips of only $-1.3 \%$ between pre-intervention ${ }^{2}$ and post-intervention ${ }^{3}$ periods (Figure 13). Between these same periods, NZHTS weighted data showed a change of $-8.0 \%$ for a sample of small cities ${ }^{4}$ compared to $-5.6 \%$ for the intervention cities. The larger decline in walking rates in the sample of small cities in comparison to the intervention cities suggests that this is a wider issue for small cities in New Zealand. As a result, declining rates of walking in the intervention cities will not be attributed to the intervention.

\footnotetext{
${ }^{1}$ Auckland, Christchurch, Dunedin, Hamilton, Lower Hutt, Tauranga (sampled cities with over 100,000 residents)

${ }^{2}$ 2003-2009

${ }^{3} 2010-2013$

${ }^{4}$ Masterton was not sampled in the NZHTS, thus a sample of small cities has been chosen to be analysed which includes: Gisborne, Invercargill, Napier, Nelson, Palmerston North, Rotorua, Whanganui and Whangarei (sampled cities with less than 100,000 residents). Te Awamutu, Upper Hutt, Cambridge and Kapiti were also sampled in the NZHTS, but were excluded due to their close proximity to large cities.
} 


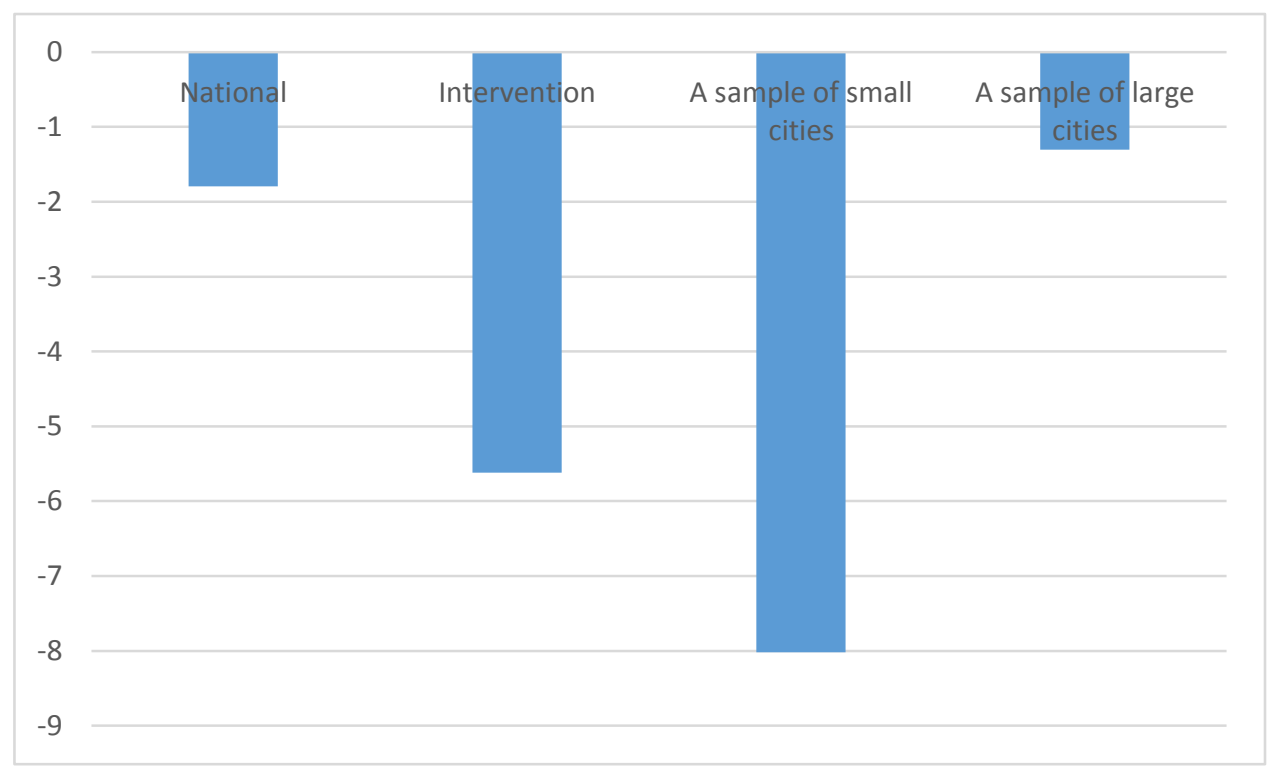

Figure 13:\% change of mean distances walked based on weighted data from NZHTS surveys for two periods: 2003-2009 and 2010-2012

Table 13 also shows Census data for cycling trips. Between 2006 and 2013, the number of cycling to work trips has increased at a higher rate for the intervention cities $(20.7 \%)$ than nationally $(13.1 \%)$, while the control cities continue to see a decline in cycling rates (13.5\%). Furthermore, analysis of weighted NZHTS data (Figure 14) shows that between the pre and post-intervention periods, cycling has declined by $-33.5 \%$ in the sample of small cities, while increasing at a rate of $26.7 \%$ in the sample of large cities. The NZHTS data shows cycling increasing by $79.2 \%$. It is worth noting that prior to 2008 , the NZHTS sample size was likely too small to accurately estimate cycling rates and distances in the intervention cities during these pre-intervention years; thus the large increase in cycling for the intervention cities could be overstated, although as Census data have shown, would have likely increased at a higher rate than the other investigated areas. As a result, increases in cycling rates reported for the intervention cities have been attributed to the intervention. 


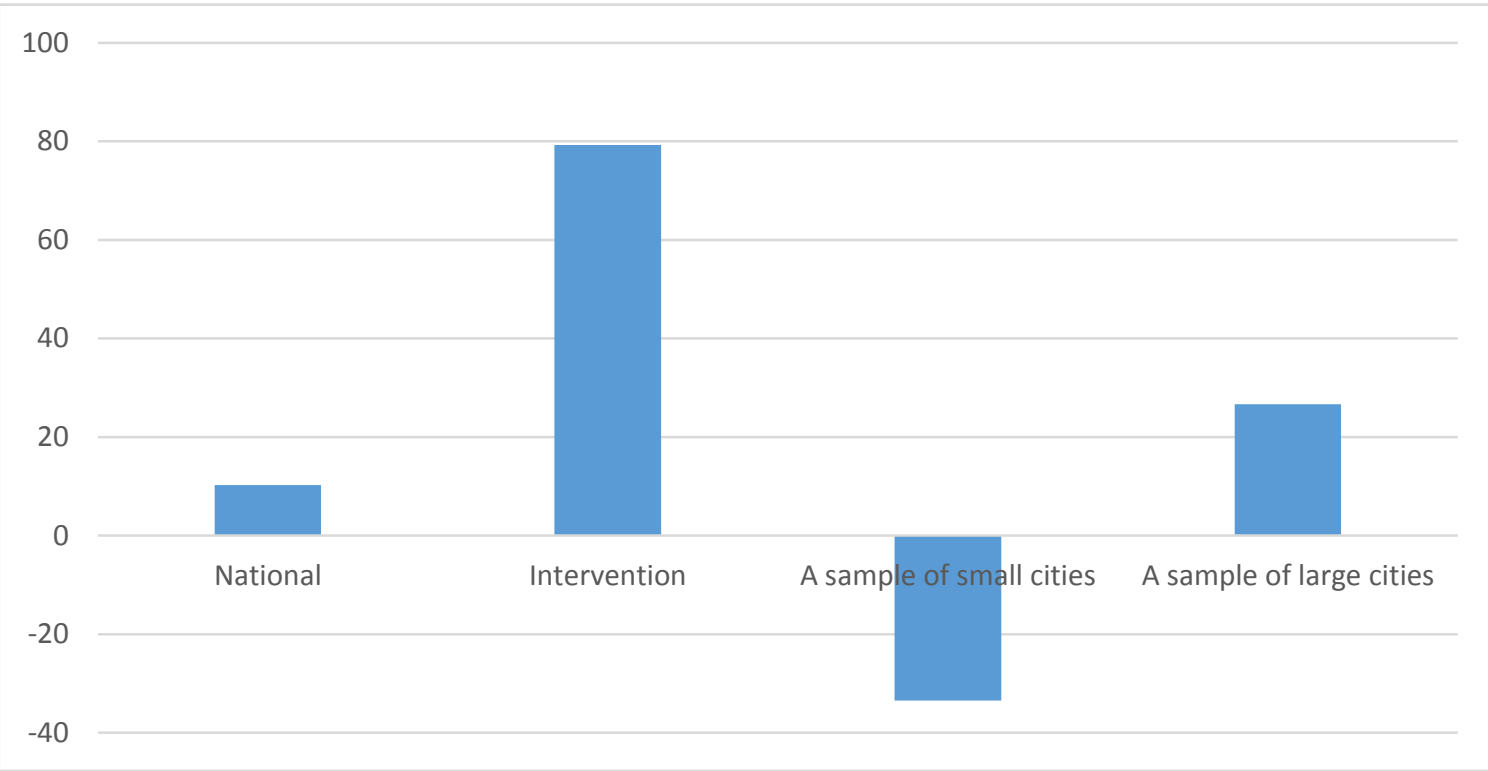

Figure 14: \% change of mean distances cycled based on weighted data from NZHTS surveys for two periods: $2003-2009$ and 2010-2012

Declining rates of cycling in both the control cities (Table 13) and the sample of small cities (as seen in Figure 14) may suggest that an intervention that is able to diminish or reverse declining rates of walking or cycling could be credited with both the number of additional trips and users gained as a result of the intervention, as well as the number of estimated trips and users that would have been lost in the absence of the intervention.

\begin{tabular}{|llllll|}
\hline & Intervention & \multicolumn{3}{c|}{ Control } \\
& & Per year & Baseline adjusted & Per year \\
& \% change & average & average & \% change & average \\
\hline $2001-2006$ & 11.08 & 2.22 & -- & 2.73 & 0.55 \\
\hline $2006-2013$ & -6.59 & -0.94 & -1.65 & -17.68 & -2.53 \\
\hline
\end{tabular}

Table 14: Walking growth estimates based on 2001-2013 Census travel to work data

Census data provide values for 2013 that conveniently coincide with the end of the initial intervention period. However, the prior Census (2006) is three years before the baseline year of this evaluation (2009). To estimate the baseline for the intervention cities, this evaluation uses the per year average \% change value between the 2001 and 2006 census dates; thus the 2007-2009 estimated annual growth rates for walking are set at 2.22\% (Table 14). The 2010-2013 estimated annual growth rates for walking are set at $-1.65 \%$, 
the baseline adjusted average ${ }^{1}$. It is assumed that this decline in walking rates is the result of a wider trend irrespective of the intervention, although it is worth mentioning that this negative trend is observed to a higher degree nationally and in the control cities. The rate of $-1.65 \%$ is set for the remainder of the evaluation timeframe, although this evaluation does not attribute the decline in walking as a cost as it reflects a wider trend irrespective of the intervention.

The intervention Census walking to work data are also set to the control conditions. In this case, the intervention trips to work figure is set to decrease at $-2.53 \%$, the per year average decline in walking observed in the control cities from 2006 to 2013 . As this rate is higher than the baseline adjusted rate observed in the intervention cities, the approach allows for an estimation of the number of trips and users that likely would have been lost in the absence of the intervention.

\begin{tabular}{|llllll|}
\hline & Intervention & & & \multicolumn{3}{c|}{ Control } \\
& & Per year & Baseline adjusted & $\%$ & Per year \\
& \% change & average & average & change & average \\
\hline $2001-2006$ & -6.13 & -1.23 & -- & -25.48 & -5.10 \\
\hline $2006-2013$ & 20.71 & 2.96 & 5.18 & -13.55 & -1.94 \\
\hline
\end{tabular}

Table 15: Cycling growth estimates based on 2001-2013 Census travel to work data

The cycling pre-intervention growth rates were set in the same manner as was done for the walking values. The post-intervention growth rates are set at the baseline adjusted average (Table 15) for five years (2010-2014), assuming that this period will experience peak growth values as a result of the newly built infrastructure and concentrated promotional and education efforts. For a ten year period starting from 2015, growth rates are estimated to diminish at three different rates: $30 \%$ (medium estimate), 20\% (high estimate) and 40\% (low estimate). At 2024 the values are fixed, representing a small rate of growth, representing the likelihood of youth and new residents taking up cycling.

\footnotetext{
${ }^{1}$ This baseline estimate applies to the four years after the intervention starting at 2010 (a period in which the growth is likely to have occurred as a result of the intervention); the baseline calculation is $-6.59 \% / 4=-1.65 \%$ per year.
} 
As for walking, the intervention Census cycling to work data are also assessed relative to the control cycling conditions. In the absence of an intervention, the rate of cycling is expected to decrease at the 2006-2013 per year average calculated from the control sample (-1.94\%). Detailed Census values and walking and cycling growth estimates can be found in Appendix C.

As the census data only provides a figure for the number of individuals who walked or cycled to work on a single day in March, estimates must be made for non-work related trips, average trip distance, number of regular users and number of days walked/cycled per year. Estimates for non-work related trips were calculated using data from the 20092012 NZHTS. Using data from this survey regarding trip purposes per mode of travel, it can be estimated that travel to work trips represent $20 \%$ of total reported walking trips and $26 \%$ of total reported cycling trips. These figures were applied to the census travel to work values to estimate the year by year numbers of trips for non-work related purposes.

Average trip distance for the baseline year (2009) was based on calculations using NZHTS weighted data. Pre-intervention data show that the average trip distance was 0.85 $\mathrm{km}$ for walkers and $2.57 \mathrm{~km}$ for cyclists. Post-intervention data suggest that average trip distance was $0.86 \mathrm{~km}$ for walkers and $3.99 \mathrm{~km}$ for cyclists (in the intervention cities). The significantly lower pre-intervention figure for cyclists is likely affected by sampling error, as the sample population for cyclists before 2008 was very small. Use of this figure would potentially overestimate post-intervention benefits; thus this study has chosen to use the post-intervention average trip distance estimates for walking and cycling for both periods.

This study calculates average daily and yearly distances walked and cycled. These calculations do not provide much insight into the number of trips being taken by regular users, who stand to achieve larger benefit shares, notably in health outcomes. A report by Krizek et al. (2006) offers the following formula to estimate the number of "everyday", or regular users of cycling infrastructure (p.A-5):

$$
\begin{aligned}
& A=0.3 \%+1.5 * C \\
& \quad \text { where } A=\% \text { of adult population who bicycle in a day } \\
& C=\text { bicycle commute share } \%
\end{aligned}
$$

The authors assume that the bicycle commute share represents the minimal number of regular cyclists, with the calculation above providing a more likely estimate. The value 
derived from this equation was then divided by the census cycling to work share which provided a multiplier (2.29). This multiplier was used to calculate the number of regular cyclists based on the cycling to work estimates. This multiplier was also used to estimate the number of walkers. It was multiplied by $26 / 20$ (\% of cycling trips for work purposes/\% of walking trips for work purposes) to give a multiplier (2.98) to estimate the number of regular walkers from the census walking to work commute share (for the quasiexperimental study). It is important to note that Krizek et al.'s formula was designed in the United States and has been accurate in predicting the numbers of everyday cyclists in most of the cities tested; however it would be imprudent to assume it accurately represents a true estimate for the New Zealand context. No other studies providing a similar function were found in the New Zealand context, and the development of such a formula is outside the scope of this study. Given the United States' lower reported Census travel to work values compared to New Zealand, it is likely that Krizek et al.'s formula would represent a conservative estimate in the context of urban New Zealand.

The WHO's HEAT methodology default value for the number of days cycled in a year is 124, based on a study from Sweden (Kahlmeier \& The World Health Organization Regional Office for Europe, 2013). Given New Zealand's comparatively milder winters, it is likely that this value will be higher in the context of New Zealand. This study uses a value of 144 for walkers and cyclists, based on an assumption that regular users will walk or cycle to work on average 3 days a week for 48 weeks a year. This value could also provide a realistic estimate for distances walked/cycled for users who regularly walk/cycle for purposes other than work.

\subsection{Benefit analysis of the MCP}

This CBA calculates benefits using four separate evaluations, each of which contains a unique set of conditions. Evaluation \#1 closely mirrors the conditions set up in NZTA's 2010 Economic Evaluation Manual Volume \#2, which provided a standardised framework for ex ante economic appraisal of transportation projects prior to the release of NZTA's 2013 Economic Evaluation Manual (effective from July 1 ${ }^{\text {st }}$ 2013). Evaluation \#2 uses conditions outlined in NZTA’s 2013 Economic Evaluation Manual, to calculate the economic efficiency of the MCP. Evaluation \#3 is entitled "a more robust estimate" and evaluation \#4 is entitled "an optimistic estimate". Evaluation \#3 and Evaluation \#4 essentially assess the same benefits (mortality savings, health care cost savings, insecurity 
reduction benefit, greenhouse gas savings, vehicle operating cost savings, journey quality benefit, reduced cost of absenteeism, and congestion savings), with a few differing conditions of evaluation (to be explained later). These benefits have been chosen as they have been cited in previous studies as being important benefits to consider when evaluating AT interventions.

Each of the four CBA evaluations is undertaken using two different models. The first model simply looks at the perceived benefits achieved by the intervention itself. The second model adopts a quasi-experimental design, similar in nature to that which the ACTIVE study has employed (Chapman et al., 2014). This quasi-experiment compares walking and cycling rates for the intervention cities (Hastings and New Plymouth) to rates in control cities (Masterton and Wanganui). The control cities were chosen because they have similar geographies, climates and demographics to the intervention cities, but were not awarded national funding for walking and cycling programmes during the intervention time period and did not undertake significant walking and cycling investment programmes. The goal of the quasi-experiment is not only to confirm observations of increased numbers of walking and cycling trips, but also to take into account trips that may have been lost in the absence of the intervention using observed trends in the control cities. Given the national trends of diminishing rates of walking and cycling, it is reasonable to assume that an intervention that reverses this can be credited with the number of walking and cycling trips it is perceived to have retained, or 'saved'.

It is important to note that the census data do not provide information in terms of walkers' and cyclists' choice of routes. It is plausible to assume that not all trips will take place on MCP infrastructure, although the expansive nature of the new infrastructure (105 $\mathrm{km}$ of new/improved routes in both cities) likely suggests that a large portion of users would use the MCP infrastructure for their journeys. This study estimates, conservatively, that $50 \%$ of new trips and new kilometres cycled and new "regular cyclists" can be attributed to the intervention. As for walkers, $25 \%$ of estimated new trips walked, new $\mathrm{km}$ walked and new walkers are attributed to the intervention. This lower value reflects the number of new/improved facilities available to walkers being less than for cyclists, although promotional/educational efforts were made to promote both modes. Observations from the intercept surveys suggest that a large number of users for both modes are using shared pathways, and thus a significant number of walkers should be attributed to the intervention. These estimations apply to all benefit calculations across each of the four CBA evaluations. 


\subsection{Cost-benefit analysis methodologies}

\subsubsection{Cost-benefit analysis evaluation \#1, based on the New Zealand Transport Agency's 2010 Economic Evaluation Manual}

Evaluation \#1 evaluates two benefits outlined in NZTA's 2010 EEM (Volume \#2). In the 2010 EEM, NZTA provides composite benefits for walking and cycling. Each additional kilometre walked as a result of an improvement in walking facilities (in comparison to baseline values) is allotted a value of NZ $\$ 2.70$. Of this, NZ $\$ 2.60$ is considered a "health benefit" with the remaining NZ $\$ 0.10$ considered a "road traffic reduction benefit". As for cycling, a composite benefit of NZ $\$ 1.45$ has been estimated in the EEM for each additional kilometre cycled as a result of an improvement in cycling facilities. Of this, $\mathrm{NZ} \$ 1.30$ is considered to be a "health benefit", $\mathrm{NZ} \$ 0.10$ considered as a "road traffic reduction benefit", and the remaining NZ $\$ 0.05$ considered to be a "safety benefit". In addition to the composite benefit, the 2010 EEM allows for a provision of an additional safety benefit where a new/improved facility eliminates an impediment to safe walking (NZ\$2.70 per trip) and cycling (NZ\$4.35 per trip). The 2010 EEM provides little guidance in terms of how to use this benefit. For the sake of simplicity, this study applies this benefit to $14 \%$ of the trips. This figure represents the percentage of new/improved routes that are off-road shared pathways ( $15 \mathrm{~km}$ out of $105 \mathrm{~km}$ total), and thus likely perceived as being safer routes for walkers and cyclists, notably for less experienced users. In line with the 2010 NZTA EEM, these benefits are evaluated within a 30 year timeframe at an $8 \%$ discount rate.

\subsubsection{Cost-benefit evaluation \#2, based on the New Zealand Transport Agency's 2013 Economic Evaluation Manual}

Evaluation \#2 evaluates the benefits outlined in NZTA's 2013 EEM. The value and nature of these benefits are the same as those listed in the 2010 EEM. The primary difference is the timeframe and the discount rate used to evaluate these benefits. The 2013 EEM extended the timeframe from 30 years to 40 years and reduced the discount rate from $8 \%$ to $6 \%$. The use of Evaluation \#2 allows for an easy comparison of the effect of how changes in evaluation horizons and discount rates on the calculated benefits. It is important to note that the 2013 EEM states that it is not intended to be used for post hoc analysis of interventions. This study has chosen to use the 2010 and 2013 EEM frameworks for post hoc evaluation of the MCP intervention in order to compare the 
effect of the two EEMs' parameters on estimated benefits, and allow comparison to those of alternative frameworks.

\subsubsection{Cost-benefit analysis evaluation \#3, a "more robust estimate"}

Evaluation \#3 presents an alternative to NZTA's EEM framework for assessing the perceived benefits of walking and cycling interventions. Unlike the EEM's walking and cycling composite benefits, this evaluation explicitly categorises all of the benefits involved. The benefits and associated methodologies are as follows.

\section{Mortality savings}

In a landmark study in Copenhagen by Andersen, Schnohr, Schroll, \& Hein (2000), the authors found that regular commuter cyclists' relative risk for mortality was reduced by $28 \%$ in comparison to those who were not regular commuter cyclists. This study is the basis for the World Health Organization's Health Economic Assessment Tool (HEAT), a popular measure of mortality savings for walking and cycling interventions. Further studies have found that individuals who regularly walked to work have a $22 \%$ reduction of relative risk for mortality (Kahlmeier \& WHO Regional Office for Europe, 2013).

This study uses most of the basic principles of the HEAT model, with a few exceptions. The HEAT model applies the mortality savings to all users who walk and cycle as a result of the intervention. Comparing the baseline sample of the Andersen study to the NZ population, the Andersen study sample found that about $78 \%$ of those surveyed on average did less than 4 hours of light activity per week (e.g. gardening, light walking/cycling) or less than 2 hours of more vigorous activity per week (e.g. brisk walking/cycling, heavy gardening work, playing sports which make people sweaty or exhausted). The baseline sample also reported a smoking rate of almost $62 \%$. Both of these figures are considerably 'better' in the present day New Zealand context where, as noted above, the 2011-2012 Ministry of Health Household Health Survey reports that $46 \%$ of individuals are insufficiently active (less than 2.5 hours of moderate-intensity exercise a week) and that $17 \%$ of individuals are regular smokers.

Given New Zealand's comparatively favourable rates of physical fitness and smoking, this study has modified HEAT's mortality savings to better fit the New Zealand context. 
Mortality savings are only applied to $46 \%$ of those new users who are assumed to be using the MCP infrastructure (half of those estimated from census data forecasts). In line with the Andersen and Kahlmeier studies, the relative risk reductions are assumed to be $28 \%$ for new regular cyclists and $22 \%$ for new regular walkers. The mortality rate is set at 551.3 per 100,000 individuals, derived from the working age (15-64) population using the MoH's (2010) Mortality and Demographic Data report, reflecting the working-age population's mortality rate recommended by the HEAT's methodology. The value for a statistical life (VSL) is set at NZ\$3.85 million, as per the most recent MoT Social Cost of Road Crashes and Injuries update (Ministry of Transport, 2013b).

The original HEAT methodology assumes a 5 year build-up of user benefits. This study does not employ this assumption, rather the conservative figures already explained are applied to this benefit calculation to ensure this benefit is not overestimated.

\section{Health care cost savings}

In addition to calculating mortality savings, it is important to recognise the potential health care cost savings from inactive individuals becoming more active due to increasing levels of walking and cycling. A study by Genter \& NZ Transport Agency (2009) reported that on average, an insufficiently active individual becoming sufficiently active can provide health care cost savings of NZ\$624 - 1447 (2007 values). Using the low value and converting it to 2013 values $^{1}$, this study adopts a health care cost savings benefit of NZ $\$$ 726.78 per insufficiently active individual becoming sufficiently active as a result of the intervention. Like the mortality savings calculation, this benefit is applied to $46 \%$ of new regular walkers/cyclists who are assumed to be using the MCP facilities. This is to account for the fact that sufficiently active individuals who become walkers and cyclists are less likely to contribute to overall health care cost savings.

\section{Reduced insecurity benefit}

There are a wide ranging number of theories related to the influence of safety and risk, both actual and perceived, on a walker's or cyclist's decision to walk or cycle. This study adopts Hopkinson \& Wardman (1996) and Saelensmindes' (2004) conclusion that past

\footnotetext{
' using the Reserve Bank of New Zealand's inflation calculator: http://www.rbnz.govt.nz/monetary_policy/inflation_calculator/
} 
CBA practice has undervalued safety improvements as a benefit for improved walking and cycling facilities.

This study adopts Saelensminde's insecurity reduction benefit value of 2 NOK (NZ $\$ 0.44$ in 2013) ${ }^{1}$, for new $\mathrm{km}$ cycled as a result of the intervention. Improvements in walkers' facilities were not as significant for walkers as they were for cyclists. Thus this study applies half the full value for new $\mathrm{km}$ walked as a result of the intervention. Saelensminde (2004) applies this value of safety and comfort benefit to both new and existing $\mathrm{km}$ walked and cycled under the assumption that existing users' baseline usage can now be walked or cycled in less stressful environments. In order not to overestimate this benefit, this study only applies half the value of the full benefit to baseline $\mathrm{km}$ walked (NZ\$ 0.11) and cycled (NZ\$ 0.22), while applying the full benefit values to new $\mathrm{km}$ walked (NZ\$ 0.22) and cycled (NZ\$ 0.44).

\section{Greenhouse gas savings}

This issue of climate change is receiving greater policy attention as reports from agencies at the forefront of climate change science such as the Intergovernmental Panel on Climate Change (2014) release increasingly assertive evidence-based reports on the damaging impacts of climate change. Greater modal shift to walking and cycling is widely acknowledged as a means for households to reduce their $\mathrm{CO}_{2}$ footprints.

The benefit values used in this study are based on several assumptions. Car trips are made in automobiles that average 9 litres per $100 \mathrm{~km}$ driven². Each litre of petrol emits an average of 2167 grams of $\mathrm{CO}_{2}$, based on 2011 estimates from the United States Environmental Protection Agency (2011). Carbon costs should ideally be based on the social cost of carbon. However, as the values for this are contentious, we have chosen a conservative figure used by an established government organisation, the UK Department for Transport (2012), taking medium estimates for a non-traded ton of $\mathrm{CO}_{2}$ emissions, whose value ${ }^{3}$ increases every year (Appendix D). An illustrative value for 2015 is 57.72 GBP per tonne of $\mathrm{CO}_{2}$.

\footnotetext{
${ }^{1}$ Historical exchange rate found at: http://www.xe.com/currencycharts/?from=NOK\& to=NZD\&view=10Y, 2004 to 2013 NZ $\$$ conversion done via http://www.rbnz.govt.nz/monetary_policy/inflation_calculator/ ${ }^{2}$ Based on a medium size car using data provided by: http://www.energywise.govt.nz/your-vehicle/savingfuel/buying-a-vehicle

${ }^{3}$ Conversion from 2010 UK£ to $2010 \mathrm{NZ} \$$ done using values from: http://www.rbnz.govt.nz/statistics/tables/b1/
} 
This study's evaluation also assumes that (only) 25\% of new km walked and cycled attributed to the MCP intervention are replacing a car trip. This value could be influenced by several factors including purpose of trip and number of passengers (if any). $25 \%$ is assumed to be a realistic, if conservative, value.

\section{Vehicle operating costs savings}

There are several components that comprise the costs of motor vehicle ownership including costs associated with registration, maintenance, petrol, warrant of fitness, parking and the cost of the motor vehicle itself. Individuals who replace motor vehicle trips with walking and cycling trips can expect to achieve variable cost savings from driving less, and in some cases substantial savings for those users who are able to forgo motor vehicle ownership. For families, it could also mean becoming a one-car family.

The value for this benefit is taken from Pettit's (2013) per km estimation of the NZTA's 2013 EEM cost of car ownership (about NZ\$ 1.00). Like the Greenhouse Gas Savings, it is applied to $25 \%$ of new walking and cycling trips attributed to the MCP intervention.

\section{Journey quality benefit}

This benefit is intended to represent facility improvements that create a more pleasant journey for walkers and cyclists. This can include addition of more scenic or convenient routes, better surface quality (e.g. wider, smoother, less slippery), better coverage of routes or possibly even account for a more reliable form of transportation in face of motor vehicle congestion.

This benefit is taken from a study published by Wilson \& Cope (2011) on behalf of the UK Department for Transport. Per km values for walkers are estimated to be NZ $\$ 0.13^{1}$ and per $\mathrm{km}$ values for cyclists are estimated to be $\mathrm{NZ} \$ 0.37^{2}$ for cyclists. It is worth noting that the author's original intention is to apply the full value to existing $\mathrm{km}$ walked or cycled and half the value to new $\mathrm{km}$ walked or cycled. This study has inversed this process and allotted the full value to new $\mathrm{km}$ walked or cycled as a result of the

\footnotetext{
' Conversion to 2013 NZ\$ done via: http://www.rbnz.govt.nz/statistics/tables/b1/

${ }^{2}$ Original value a per min rather than a per $\mathrm{km}$ value. This value assumes an average cycling speed of $14 \mathrm{~km} / \mathrm{h}$ (as per the HEAT methodology), conversion to $2013 \mathrm{NZ} \$$ done via: http://www.rbnz.govt.nz/statistics/tables/b1/
} 
intervention under the assumption that the additional $\mathrm{km}$ are more valuable to society than the original baseline $\mathrm{km}$, most of which would have been walked or cycled regardless of the intervention. Baseline $\mathrm{km}$ walked or cycled are ascribed half of the full benefit.

\section{Reduced absenteeism savings}

A Dutch study by van Amelsvoort (2006) found that individuals who partook in active physical activity at least twice a week took on average $24 \%$ less sick leave then those individuals who were not active. In terms of the effectiveness of workplace interventions that promote physical activity, reviews by Proper \& van Mechelen (2008) and Conn, Hafdahl, Cooper, Brown, \& Lusk (2009) found positive links between workplace physical activity interventions and absenteeism. However, both reviews had concerns with study designs and as a result did not conclude a definitive correlation between workplace physical activity interventions and reduced absenteeism.

This study assumes that new walkers and cyclists attributable to the MCP intervention who were previously inactive will take on average one less sick day per year. A value of $\mathrm{NZ} \$ 168.80^{1}$ is added for $46 \%$ (the percentage of inactive adults in New Zealand) of new walkers and cyclists.

\section{Congestion savings}

Increasing rates of walking and cycling are recognised as reducing congestion in urban areas. This evaluation assumes that $25 \%$ of new walking and cycling trips attributable to the MCP intervention are replacing motor vehicle trips, as noted earlier, and thus are designated a benefit. Walkers are assigned a per $\mathrm{km}$ value of $\mathrm{NZ} \$ 0.48^{2}$, a value attributed to Litman (2004) $)^{2}$. Cyclists are given a per $\mathrm{km}$ value of $\mathrm{NZ} \$ 0.35^{2}$, a value given by SQW (2007).

\footnotetext{
${ }^{1}$ Represents average daily wages based on median daily earnings estimated found at: http://www.stats.govt.nz/browse_for_stats/income-andwork/Income/NZIncomeSurvey_HOTPJun13qtr.aspx
}

\footnotetext{
${ }^{2}$ Historical exchange rate found at: http://www.xe.com/currencycharts/?from=NOK\&to=NZD\&view=10Y,
} 2004 to 2013 NZ $\$$ conversion done via http://www.rbnz.govt.nz/monetary_policy/inflation_calculator/ 


\section{Conditions of evaluation}

This evaluation is carried out using a 40 year timeframe at a $3 \%$ discount rate. The lower discount rate is used, as discussed earlier, to better account for intergenerational equity.

\section{Benefits not included in this evaluation}

Safety in numbers is assumed to arise as a result of the intervention; thus accidents rates are assumed not to change as a result of the intervention.

Community related benefits such as liveability, walkability, improved social capital and reduced severance and barrier costs are not included. These are assumed to be important, but difficult if not impossible to meaningfully quantify. These will be addressed in Chapter 7.

\subsubsection{Cost-benefit evaluation \#4, an "Optimistic Estimate"}

This evaluation closely mirrors Evaluation \#3, with a few key differences. Benefits such as Greenhouse Gas Savings, Vehicle Operating Cost Savings and Congestion Savings are calculated assuming $50 \%$ of new walking and cycling trips attributed to the MCP intervention are replacing motor vehicle trips (versus $25 \%$ as in Evaluation \#3). The Greenhouse Gas Savings benefit also uses the UK DfT's high (rather than medium, as in Evaluation \#3) value for a Non-traded Tonne of $\mathrm{CO}_{2}$, listed in Appendix D.

The evaluation timeframe has been extended from 40 years to 50 years while the discount rate has been set at $1.4 \%$ (versus $3 \%$ in Evaluation \#3). This extended timeframe allows for a longer capture of benefits. The lower discount rate is set at Stern's (2006) recommended discount rate for interventions that mitigate climate change. In effect, this evaluation is "optimistic" in that its conditions for economic evaluation are considerably less conservative than those currently employed by NZTA. When accounting for the intergenerational issue of climate change, there is a good case for using low social discount rates, and exploring optimistic assumptions about take-up of walking and cycling. 


\subsection{Analysis of Model Communities Programme users' perceptions of benefits}

This evaluation of the Model Communities Programme not only provides an economic evaluation of the intervention, it also undertakes an evaluation of users' perceptions of the intervention's personal and community-related benefits. Economic evaluations such as CBA assess potential benefits as perceived by the evaluator(s) alone. CBA largely focuses on benefits that can be quantified and monetised, and thus provides very little opportunity to explore other benefits that cannot be monetised. Whereas Chapter 6 has provided an economic evaluation of the MCP, Chapter 7 focuses on investigating how MCP users perceive they have benefitted from the intervention. The author is unaware of any other completed active travel intervention studies that evaluate both economic benefits and users' perceptions of benefits. This chapter is designed to provide a more holistic evaluation of the MCP.

\subsubsection{Intercept survey design and survey sampling}

The intercept survey was designed to evaluate users' perceptions of how they have benefitted from the MCP. The survey itself was formatted as a semi-structured interview, with questions of both a quantitative and qualitative nature. A semi-structured interview format was chosen as it allows for collection of data that is both specific and broad in nature (Bryman, 2008). The qualitative questions in particular allowed for a breadth of responses, including responses that could not have been predicted. This author anticipated that users would provide insightful information outside of the surveys original design scope, thus this secondary data were actively recorded.

In terms of sampling, the intercept surveys took place on shared pathways that were built or improved during the MCP intervention. Shared pathways were preferred for several reasons: they allowed access to both walkers and cyclists, the surrounding environment was often quieter and they posed a much lower risk for cyclists than the alternative of onroad cycle lanes where cyclists ride in close proximity to motor vehicle traffic. In conducting the interviews, it was found that cyclists were particularly challenging to stop due to their faster travel; thus locations that limited cyclists' speed (stop gates, railroad crossings, the top of rises, bridges, tight corners) were preferred. The intercept survey attempted to sample walkers and cyclists from both Hastings and New Plymouth districts. Only walkers and cyclists who were residing in Hastings and New Plymouth in 2013 were 
surveyed. In order not to overburden participants with a long interview process, users were surveyed based on their mode of transport when stopped (e.g. users who were cycling when stopped were not asked question related to walkers, and vice-versa).

\subsubsection{Model Community Programme users' perceptions of benefits}

The intercept survey asked several benefit perception questions of a quantitative nature. Question 2b (walkers) and 4b (cyclists) asked users to estimate their average weekly time spent/distance travelled as a walker/cyclist in 2009 (if at all), the year before the MCP was implemented. There were initial concerns that users would find it difficult to recall this information from around four years ago. To mitigate this, the questions used the expression 'before iWay/Let's Go' to give the users a baseline reference point. Given the high level of user awareness of these brands, this was a helpful addition to the survey. Users were also allowed to respond in hours per week or distance in $\mathrm{km}$. This study presents the data in distances; thus responses given in hours were converted to distances using values used by the WHO's HEAT methodology ${ }^{1}$. The intercept survey also asked users to rate the facilities pre-intervention (2009) and post-intervention (2013) against six criteria (identical for walkers and cyclists) on a ten point scale (1 low, 10 high). Users were also asked to rank these six criteria, from the most important to them (1) to the least important (6). Finally, users were asked to place a value per year for walking and cycling facilities in their district.

\subsubsection{Model Communities Programme users' perceptions of lifestyle and transportation benefits}

Questions 13-15 sought qualitative personal responses regarding the MCP impacts on participant's lifestyles as well as their present and near future transportation habits. Question 16 asked users how the intervention impacted their community. Perceived community benefits are potentially numerous and notoriously difficult to value, and thus have regularly been overlooked in economic evaluation of transportation projects (Litman, 2013a). These questions solicited a wide range of qualitative responses, of which the most common and the most insightful are analysed and presented in this study.

${ }^{1} 4.8 \mathrm{~km} / \mathrm{h}$ for walkers, $14 \mathrm{~km} / \mathrm{h}$ for cyclists 
In addition to analysis of these qualitative responses, Chapter 7 also provides several personal user narratives given that users benefit from active travel interventions in different ways. Some users' circumstances and viewpoints can be exceptional, yet economic evaluation is structured to overlook these users' special circumstances (as well as dissenting viewpoints) in favour of providing valuations and analysis at the aggregate level. Elliott (2005) argues that narrative analysis can act as a bridge between quantitative and qualitative methodologies; the author further argues:

By attending to the narrative properties of data, by using narrative to inform our analysis, and, most importantly, by recognizing ourselves as the narrators of sociological accounts, we are forced to examine our own role in the construction and maintenance of the social world. (p.187)

In other words, narrative analysis can provide potentially critical insight that is otherwise lost when data are aggregated and analysed at a population level.

A full copy of the intercept survey is in Appendix E.

\subsubsection{Model Community Programme project managers' perceptions of the intervention's successes and weaknesses}

Whereas Chapter 7 focuses on users' perceptions of benefits, Chapter 8 provides a brief analysis of MCP project managers' perceptions of the intervention's successes and weaknesses. Project managers from both Hastings and New Plymouth were interviewed using the interview schedule provided in Appendix F. Chapter 8 focuses its analysis on the questions related to project managers' view of their cities' respective MCP interventions. As a result of the detailed information that this interview needed to collect, the project managers received the interview schedules a week in advance. As this process involved collecting sensitive data that could potentially reflect upon the project managers' job performances, the interview was structured to ensure a fair interview process. A draft of this chapter was sent to both project managers to ensure their responses were accurately and fairly summarised.

The goal of Chapter 8 is to provide another layer of analysis to enrich the evaluation of the MCP. Project managers were intimately involved with the intervention from application to completion, and both project managers continue to work on walking and cycling programmes beyond the initial MCP intervention period. The in-depth interview 
approach thus allows for potentially insightful feedback to future project managers of active travel interventions. 
Chapter 6 - Results of the cost-benefit analysis of the Model Communities Programme

This chapter summarises the results of the economic evaluation of the MCP. The results are presented for all four CBA evaluations. Each evaluation is presented with a benefitto-cost ratio (BCR) calculated from the intervention itself (no control), as well as a BCR calculated using the quasi-experimental design, based on comparing census data trends from the intervention cities (Hastings and New Plymouth) to two control cities (Masterton and Whanganui). In each case, three estimates are provided: medium, high and low. The medium estimates are also presented using charts to illustrate the contribution of each benefit to the overall analysis of benefits. The results of these CBA evaluations are discussed further in Chapter 9. 


\subsection{CBA \#1 (based on NZTA 2010 EEM framework)}

\begin{tabular}{|lrlr|}
\hline & \multicolumn{1}{l}{ Medium } & \multicolumn{1}{l}{ High } & \multicolumn{1}{l|}{ Low } \\
\hline Composite benefits & $19,928,344$ & $23,233,656$ & $18,115,385$ \\
\hline Improved route safety & $8,452,465$ & $8,800,393$ & $8,261,627$ \\
\hline Benefits total & & & \\
Cost total & $28,380,809$ & $32,034,049$ & $26,377,012$ \\
\hline BCR & $21,092,573$ & $21,092,573$ & $21,092,573$ \\
\hline
\end{tabular}

Table 16: CBA \#1 without control, using NZTA EEM 2010 guidelines (30 years, 8\% discount rate)

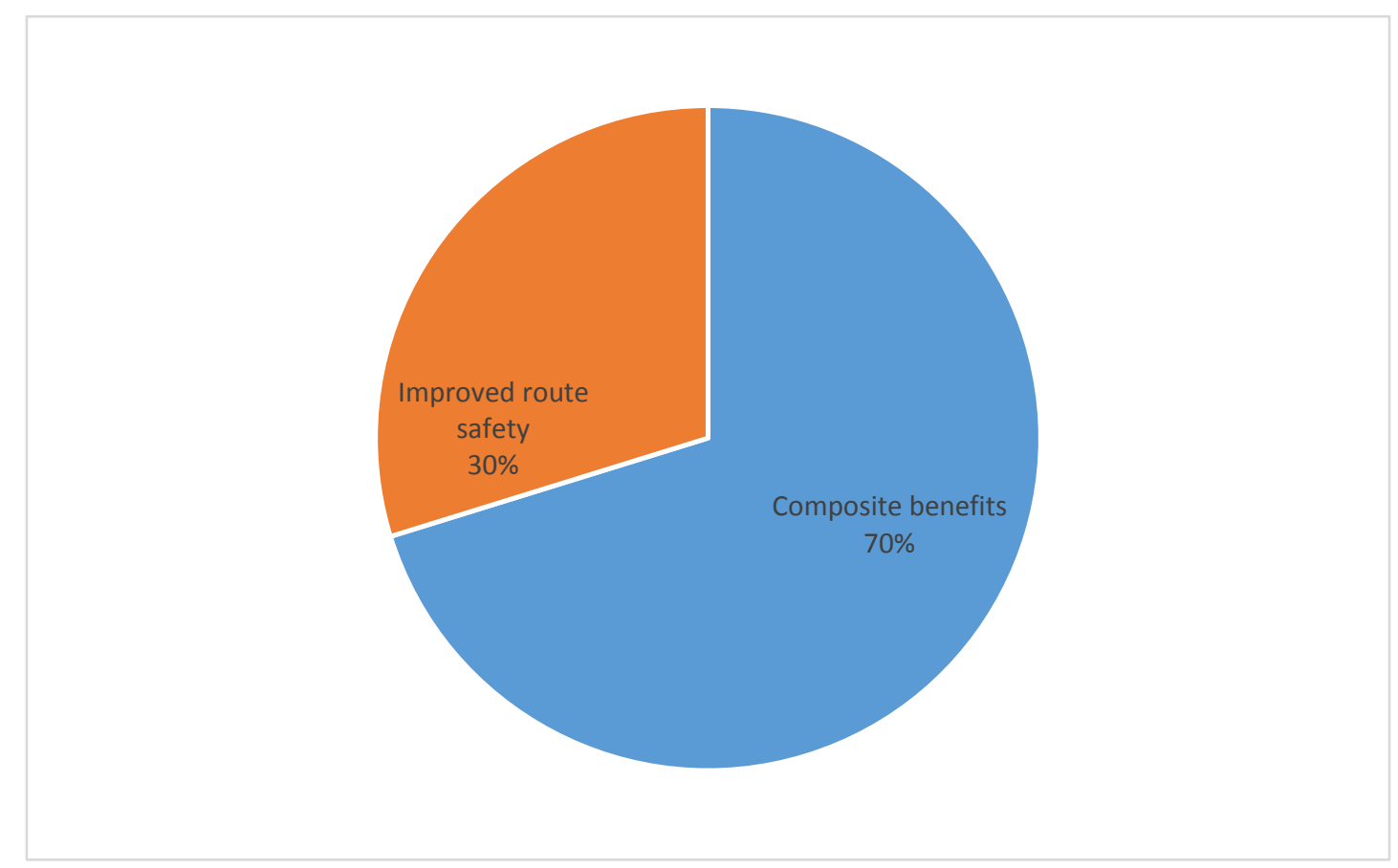

Figure 15: Distribution of benefits for CBA \#1, medium estimate, no control 


\begin{tabular}{llll} 
& Medium & High & Low \\
Composite benefits & $50,202,590$ & $53,973,608$ & $43,737,065$ \\
Improved route safety & $11,641,526$ & $12,337,381$ & $11,259,850$ \\
& & & \\
Benefits total & $61,844,116$ & $66,310,989$ & $54,996,915$ \\
Cost total & $21,092,573$ & $21,092,573$ & $21,092,573$ \\
& & & \\
BCR & 2.9 & 3.1 & 2.6 \\
\hline
\end{tabular}

Table 17: CBA \#1 with control, using NZTA EEM 2010 guidelines (30 years, $8 \%$ discount rate)

Figure 16: Distribution of benefits for CBA \#1, medium estimate, with control 


\subsection{CBA \#2 (based on NZTA 2013 EEM framework)}

\begin{tabular}{lrrlr|} 
& Medium & High & Low \\
Composite benefits & $28,415,317$ & $34,627,113$ & $25,340,528$ \\
Improved route safety & $11,327,072$ & $11,980,945$ & $11,003,410$ \\
& & & \\
Benefits total & $39,742,389$ & $46,608,058$ & $36,343,938$ \\
Cost total & $22,947,291$ & $22,947,291$ & $22,947,291$ \\
& & & \\
BCR & 1.7 & 2.0 & 1.6 \\
\hline Table 18: CBA \#2, without control, based on NZTA 2013 EEM framework (40 years, 6\% discount rate)
\end{tabular}

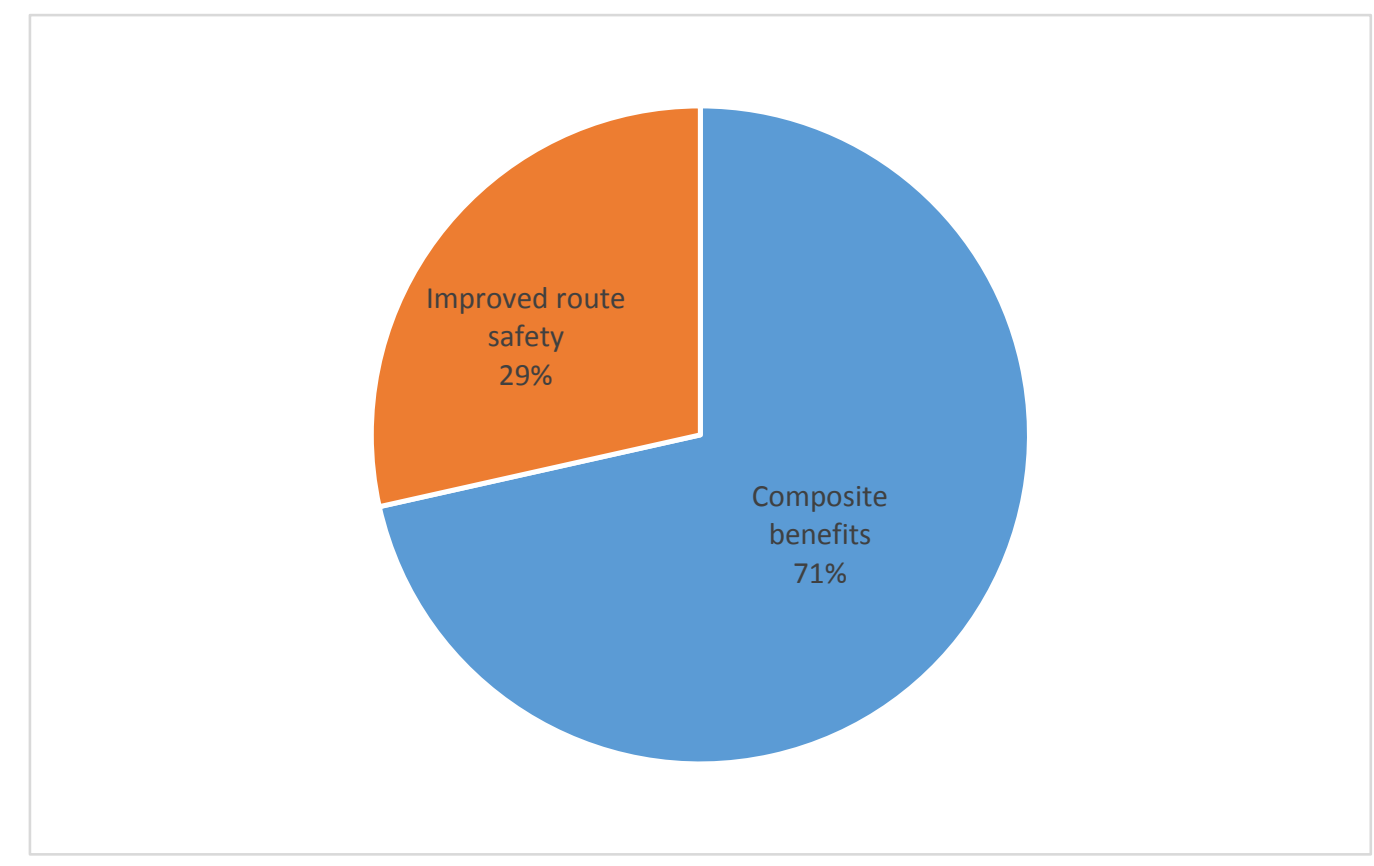

Figure 17: Distribution of benefits for CBA \#2, medium estimate, no control 


\begin{tabular}{|l|l|l|l|}
\hline & Medium & High & Low \\
\hline Composite benefits & $68,921,726$ & $81,345,317$ & $62,772,148$ \\
\hline Improved route safety & $16,112,271$ & $17,420,018$ & $15,464,947$ \\
\hline Benefits total & & & \\
\hline Cost total & $85,033,997$ & $98,765,335$ & $78,237,095$ \\
\hline BCR & $22,947,291$ & $22,947,291$ & $22,947,291$ \\
\hline
\end{tabular}

Table 19: CBA \#2, with control, based on NZTA 2013 EEM framework (40 years, 6\% discount rate)

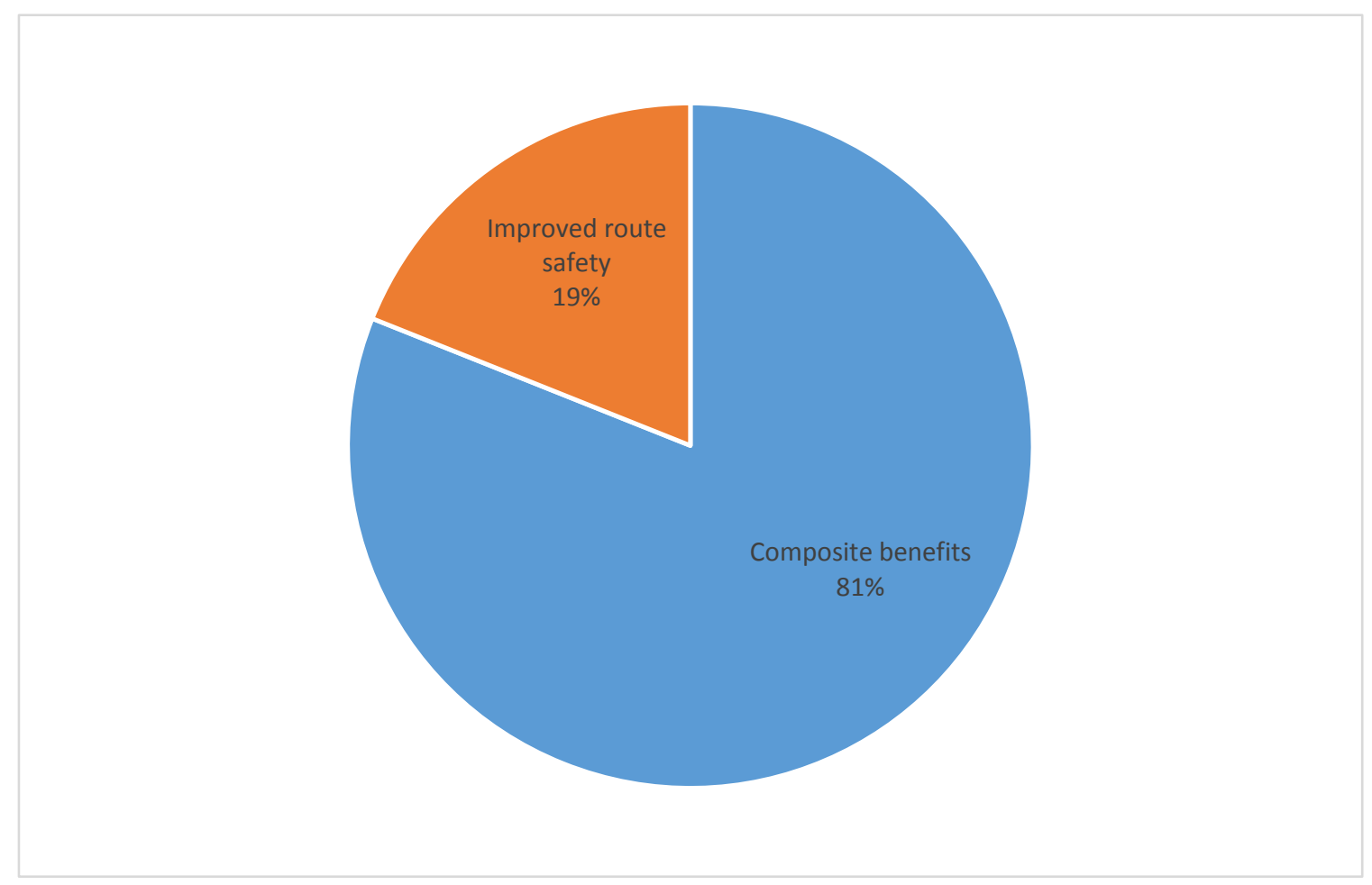

Figure 18: Distribution of benefits for CBA \#2, medium estimate, with control 


\subsection{CBA \#3 (More Robust Estimate)}

\begin{tabular}{|l|r|r|r|}
\hline Mortality savings & Medium & High & Low \\
\hline Health care savings & $45,051,398$ & $56,975,210$ & $39,478,790$ \\
\hline $\begin{array}{l}\text { Insecurity reduction } \\
\text { benefit }\end{array}$ & $5,509,402$ & $6,967,583$ & $4,827,920$ \\
\hline Greenhouse gas savings & $31,920,890$ & $35,622,278$ & $30,191,042$ \\
\hline $\begin{array}{l}\text { Vehicle operating cost } \\
\text { savings }\end{array}$ & 300,086 & 399,048 & 257,234 \\
\hline Journey quality benefit & $26,842,567$ & $29,955,098$ & $25,387,922$ \\
\hline Reduced absenteeism & $1,279,599$ & $1,618,272$ & $1,121,320$ \\
\hline Congestion savings & $2,781,078$ & $3,517,149$ & $2,437,074$ \\
\hline & & & \\
\hline Benefits total & $121,623,011$ & $145,093,589$ & $110,657,407$ \\
\hline Costs total & $22,947,291$ & $22,947,291$ & $22,947,291$ \\
\hline BCR & & & 4.8 \\
\hline
\end{tabular}

Table 20: CBA \#3, without control, more robust estimate (40 years, $3 \%$ discount rate)

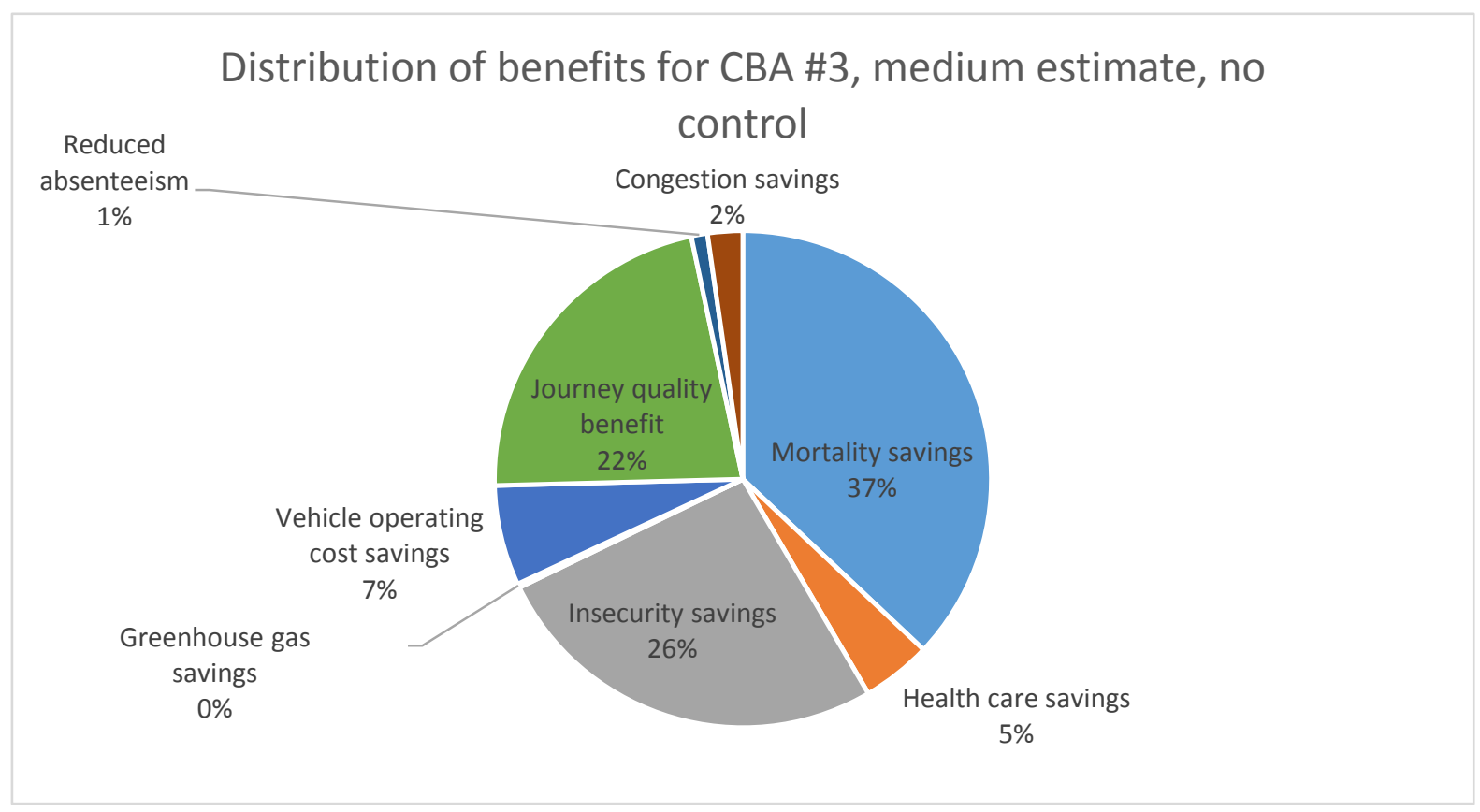

Figure 19: Distribution of benefits for CBA \#3, medium estimate, without control 


\begin{tabular}{|lrrr|} 
& \multicolumn{1}{l}{ Medium } & \multicolumn{1}{l}{ High } & \multicolumn{1}{l}{ Low } \\
\hline Mortality savings & $102,553,720$ & $126,401,345$ & $91,408,504$ \\
\hline Health care savings & $11,950,815$ & $11,903,259$ & $10,587,850$ \\
\hline Insecurity reduction benefit & $57,275,301$ & $64,678,078$ & $53,815,605$ \\
\hline Greenhouse gas savings & 792,545 & 706,842 & $9,964,058$ \\
\hline Vehicle operating cost & & & \\
savings & $20,334,137$ & $24,536,054$ & $18,370,366$ \\
\hline Journey quality benefit & $47,661,483$ & $53,886,546$ & $44,752,193$ \\
\hline Reduced absenteeism & $2,775,665$ & $3,453,011$ & $2,459,106$ \\
Congestion savings & $7,012,685$ & $2,828,114$ & $6,324,678$ \\
\hline Benefits total & & & \\
Costs total & $250,356,353$ & $288,393,249$ & $237,682,361$ \\
\hline BCR & $22,947,291$ & $22,947,291$ & $22,947,291$ \\
\hline
\end{tabular}

Table 21: CBA \#3, with control, more robust estimate (40 years, 3\% discount rate)

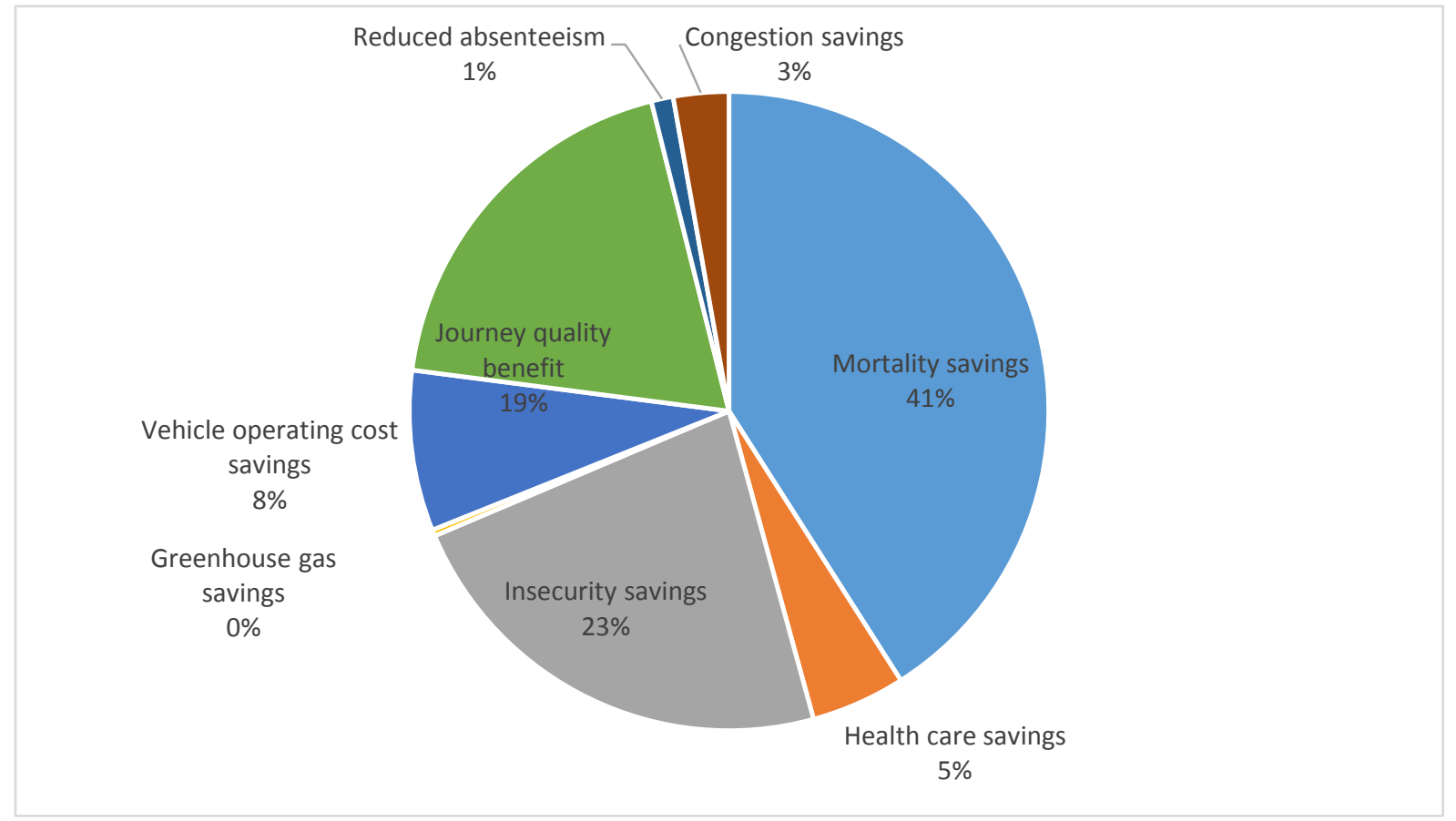

Figure 20: Distribution of benefits for CBA \#3, medium estimate, with control 


\subsection{CBA \#4 (Optimistic Estimate)}

\begin{tabular}{|l|r|r|r|}
\hline & \multicolumn{1}{|c}{ Medium } & \multicolumn{1}{l}{ High } & \multicolumn{1}{l}{ Low } \\
\hline Mortality savings & $74,281,004$ & $100,307,068$ & $63,395,828$ \\
\hline Health care savings & $9,083,934$ & $12,266,700$ & $7,752,770$ \\
\hline Insecurity reduction benefit & $50,394,975$ & $58,473,983$ & $47,016,000$ \\
\hline Greenhouse gas savings & $2,005,853$ & $2,907,701$ & $1,660,015$ \\
\hline Vehicle operating cost & & & \\
savings & $13,088,205$ & $17,673,960$ & $11,170,253$ \\
\hline Journey quality benefit & $42,377,593$ & $49,171,304$ & $39,536,182$ \\
\hline Reduced absenteeism & $2,109,811$ & $2,849,031$ & $1,800,638$ \\
\hline Congestion savings & $9,170,915$ & $12,384,156$ & $7,827,004$ \\
\hline & & & \\
\hline Benefits total & & & \\
\hline Costs total & $202,512,290$ & $256,033,904$ & $180,158,690$ \\
\hline BCR & $24,796,353$ & $24,796,353$ & $24,796,353$ \\
\hline Table 22: CBA \#4, without control, optimistic estimate (50 years, 1.4\% discount rate) & \\
\hline
\end{tabular}

Table 22: CBA \#4, without control, optimistic estimate (50 years, $1.4 \%$ discount rate)

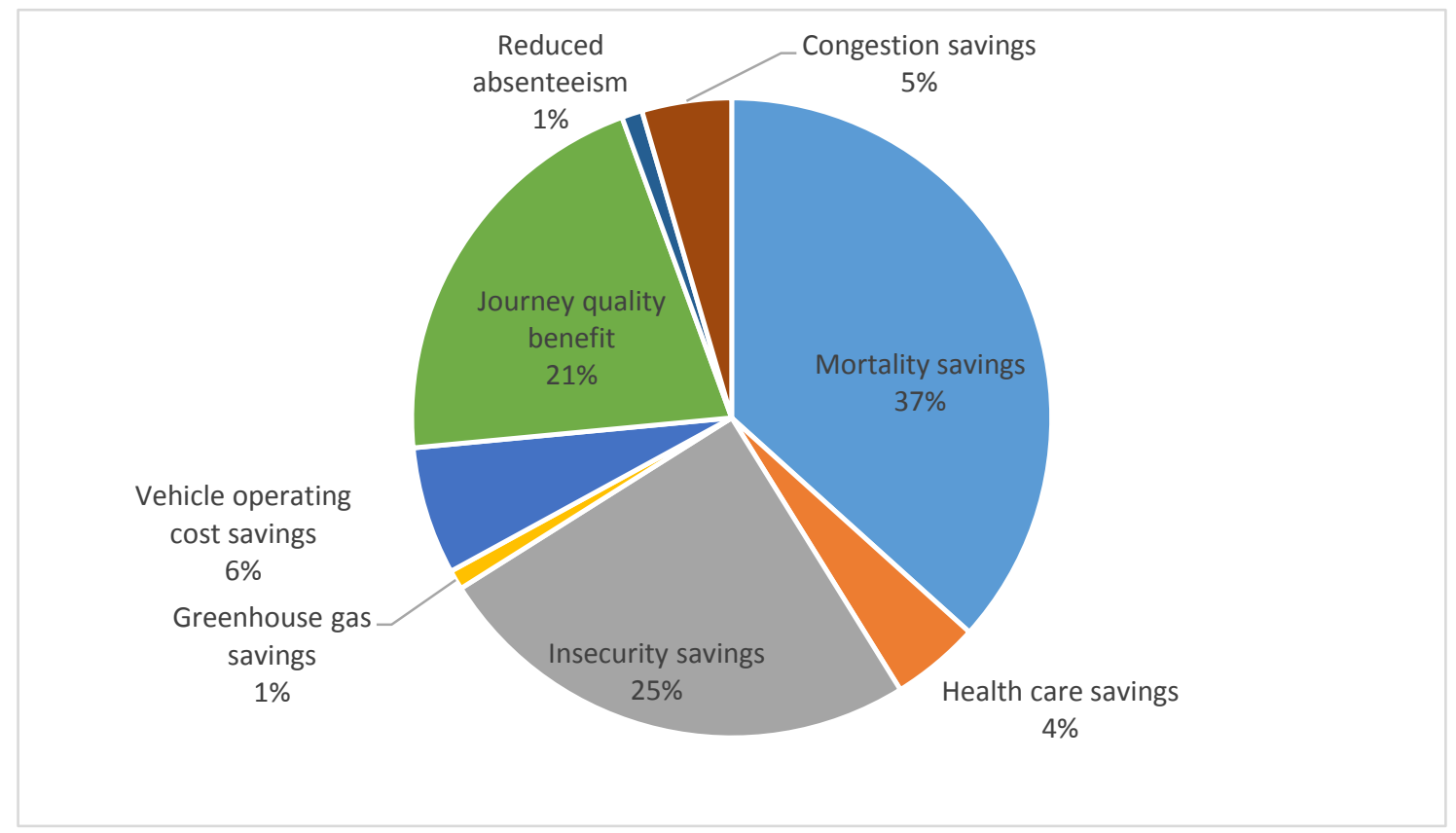

Figure 21: Distribution of benefits for CBA \#4, medium estimate, no control 


\begin{tabular}{lrrr} 
& \multicolumn{1}{l}{ Medium } & \multicolumn{1}{l}{ High } & \multicolumn{1}{l}{ Low } \\
Mortality savings & $170,456,827$ & $222,508,956$ & $148,686,475$ \\
Health care savings & $19,621,182$ & $25,986,714$ & $16,958,854$ \\
Insecurity reduction benefit & $94,289,993$ & $110,448,008$ & $87,532,043$ \\
Greenhouse gas savings & $5,539,553$ & $7,343,249$ & $4,847,876$ \\
Vehicle operating cost & & & \\
savings & $32,953,431$ & $42,124,941$ & $29,117,527$ \\
Journey quality benefit & $78,627,869$ & $92,215,291$ & $72,945,048$ \\
Reduced absenteeism & $4,557,164$ & $6,035,606$ & $3,938,818$ \\
Congestion savings & $23,871,923$ & $30,298,407$ & $21,184,103$ \\
& & & \\
Benefits total & $429,917,943$ & $536,961,172$ & $385,210,743$ \\
Costs total & $24,796,353$ & $24,796,353$ & $24,796,353$ \\
& & & \\
BCR & 17.3 & 21.7 & 15.5 \\
\hline Table 23: CBA \#4, with control, optimistic estimate (50 years, 1.4\% discount rate)
\end{tabular}

Table 23: CBA \#4, with control, optimistic estimate (50 years, $1.4 \%$ discount rate)

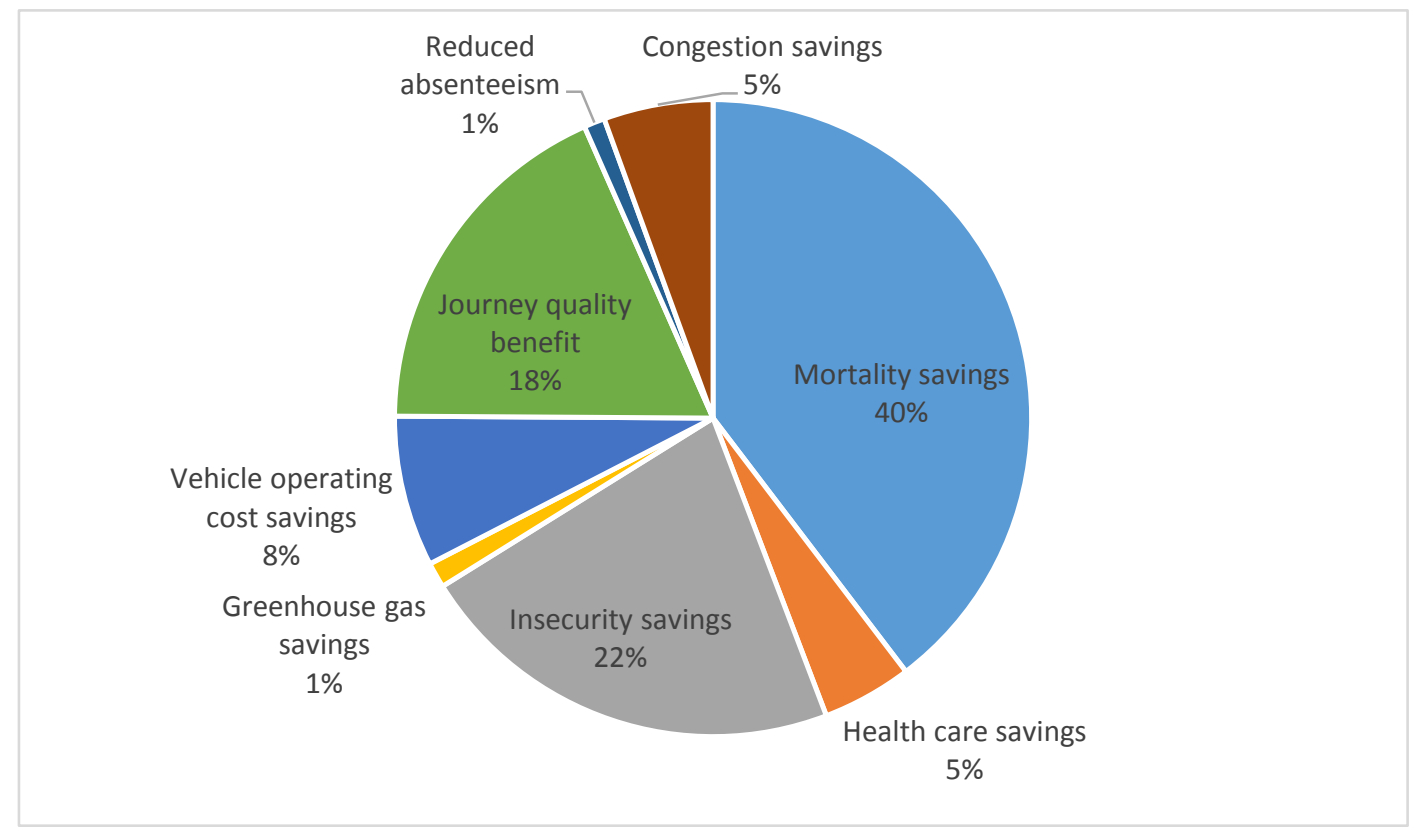

Figure 22: Distribution of benefits for CBA \#4, medium estimate, with control 


\section{Chapter 7 - Analysis of Model Community Programme users' perceptions of benefits}

Chapter 6 undertook an economic evaluation, specifically a cost-benefit analysis, of the perceived potential benefits of the Model Communities Programme. As noted earlier, in order to provide a more holistic evaluation of the potential benefits of the MCP, this study includes a mixed methods analysis exploring users' perceptions of benefits. This analysis includes evaluation of some benefits that are difficult to monetise in any meaningful way; several of these were excluded from the CBA. One exception to this is safety, a benefit whose value is frequently debated both nationally and internationally. This mixed methods analysis provides additional analysis of the importance of safety for walkers and cyclists in the New Zealand context.

This analysis is drawn from intercept survey data. These intercept surveys took place in Hastings District Council and New Plymouth District Council in December of 2013, over a one week period in each case. Walkers and cyclists were surveyed at several locations in each district, all of them on separated pathways shared between walkers and cyclists, on or adjacent to infrastructure built as a result of the MCP. A copy of the intercept survey can be found in Appendix E.

\subsection{Sample selection}

A total of 165 users were surveyed, of whom 94 were in New Plymouth and 71 in Hastings (Figure 23). 38\% of respondents identified themselves as female while $62 \%$ identified themselves as male (Figure 25), leaving males as an over-represented proportion in comparison to recent census data (Figure 24). As seen in Figure 26, the distribution of respondents is similar to the age distribution from the 2013 Census data, although it is potentially under-representing 10-19 and 20-29 age groupings while potentially over- representing the 30-39 and 60-69 age groupings. Given the higher number of cyclists in the sample population, it is not surprising to see that the 70+ age grouping represents a small proportion of respondents surveyed. 


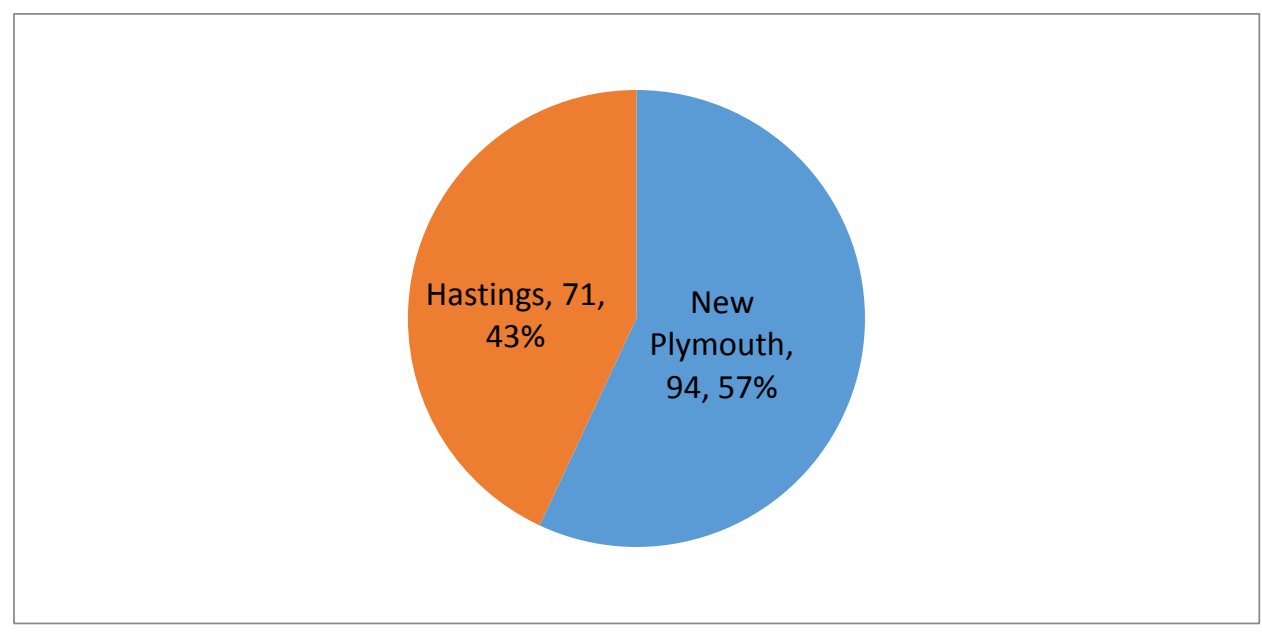

Figure 23: MCP intercept surveys, respondents surveyed by district council, $n=165$

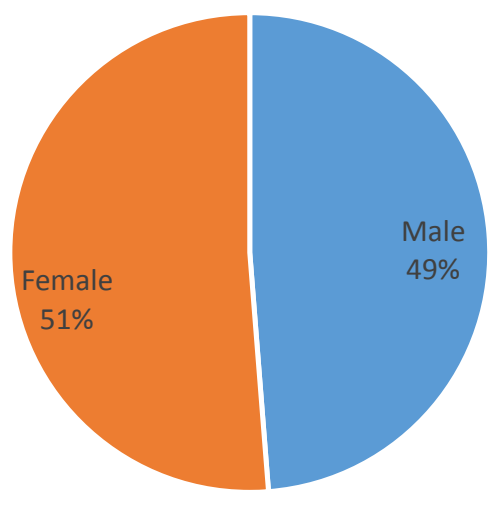

Figure 24: 2006 Census population by sex for Hastings and New Plymouth districts (Stats New Zealand)

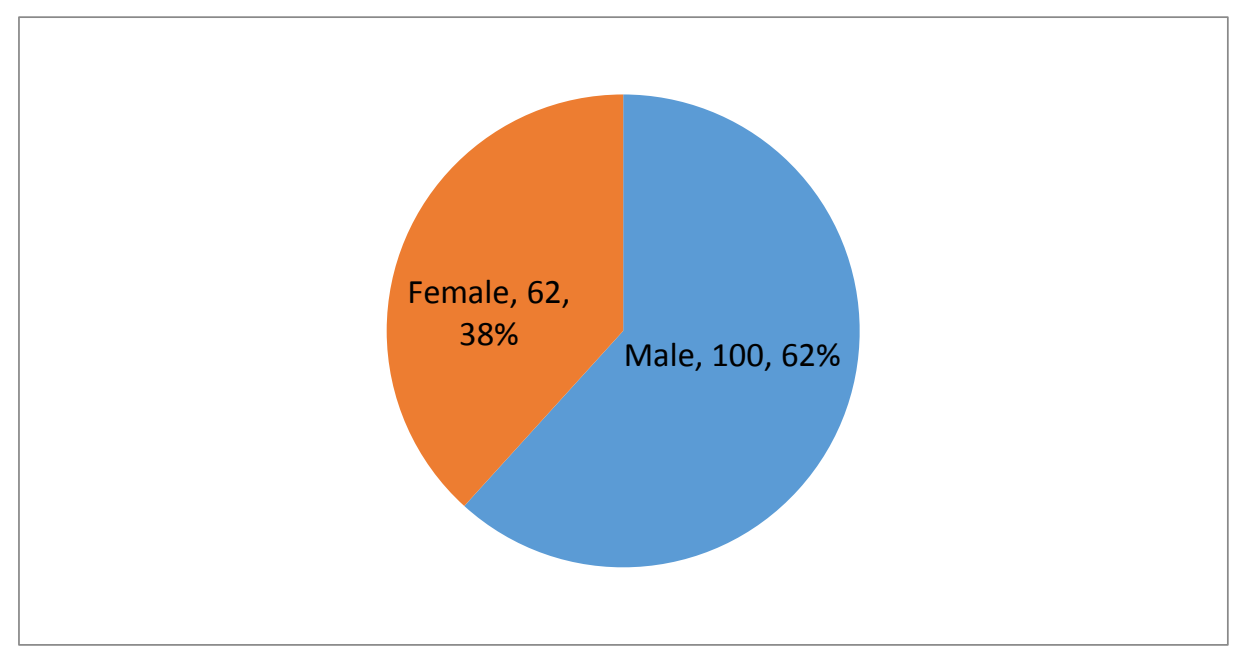

Figure 25: MCP intercept survey respondents by gender $(n=162)$ 


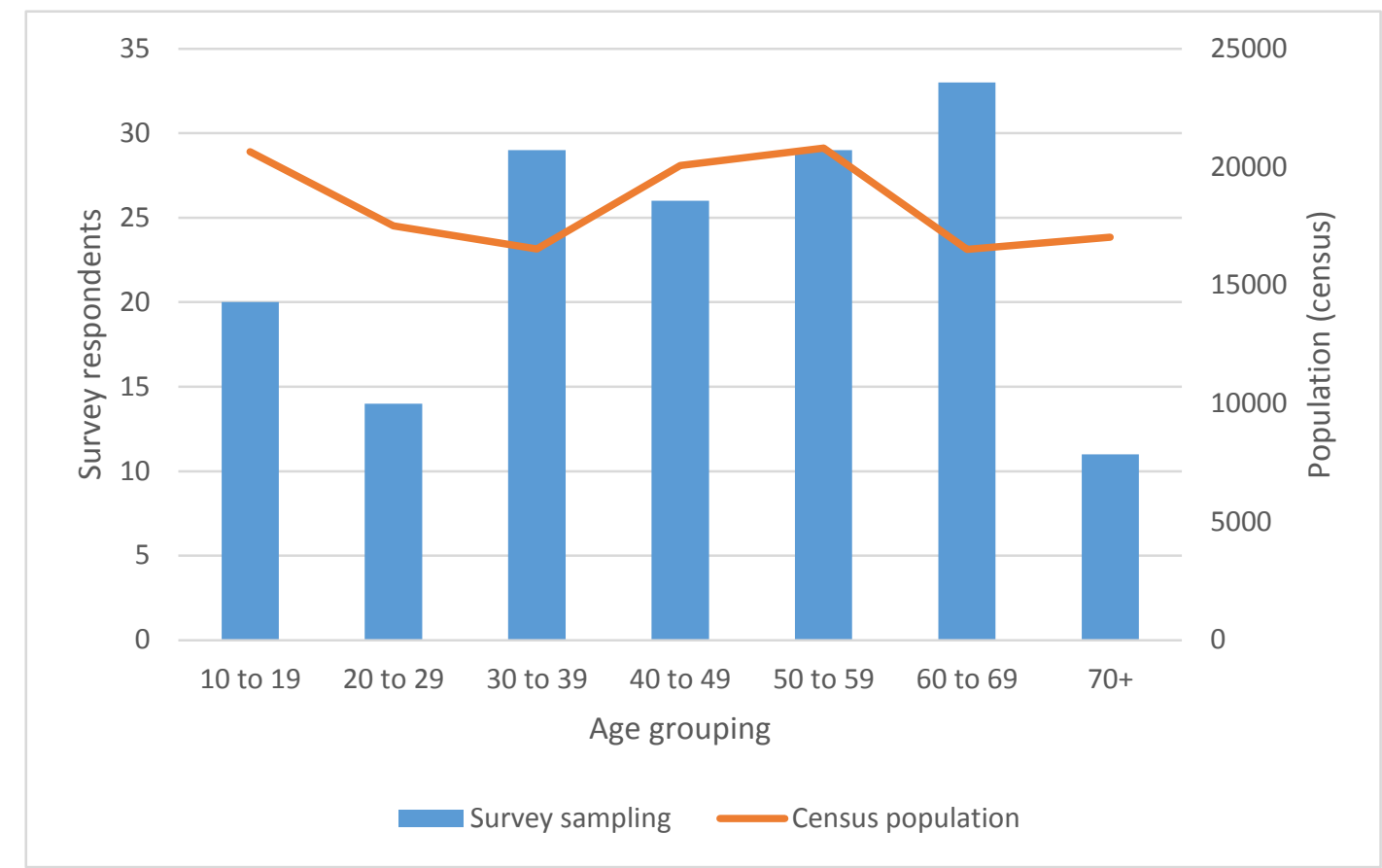

Figure 26: MCP intercept survey sampling by age group compared to 2013 Census population by age grouping for Hastings \& New Plymouth districts

A total of 158 respondents provided 164 responses for ethnicity (respondents could nominate more than one ethnicity). $86 \%$ of respondents identified themselves as European (76\% in 2006 Census) while 7\% identified themselves as Maori (18\% in 2006 Census). A small percentage of respondents identified themselves as from Asian (2\%) or Pasifika (1\%) ethnicity. At the time respondents were interviewed, 90 (55\%) were cycling while 75 (45\%) were walking. 


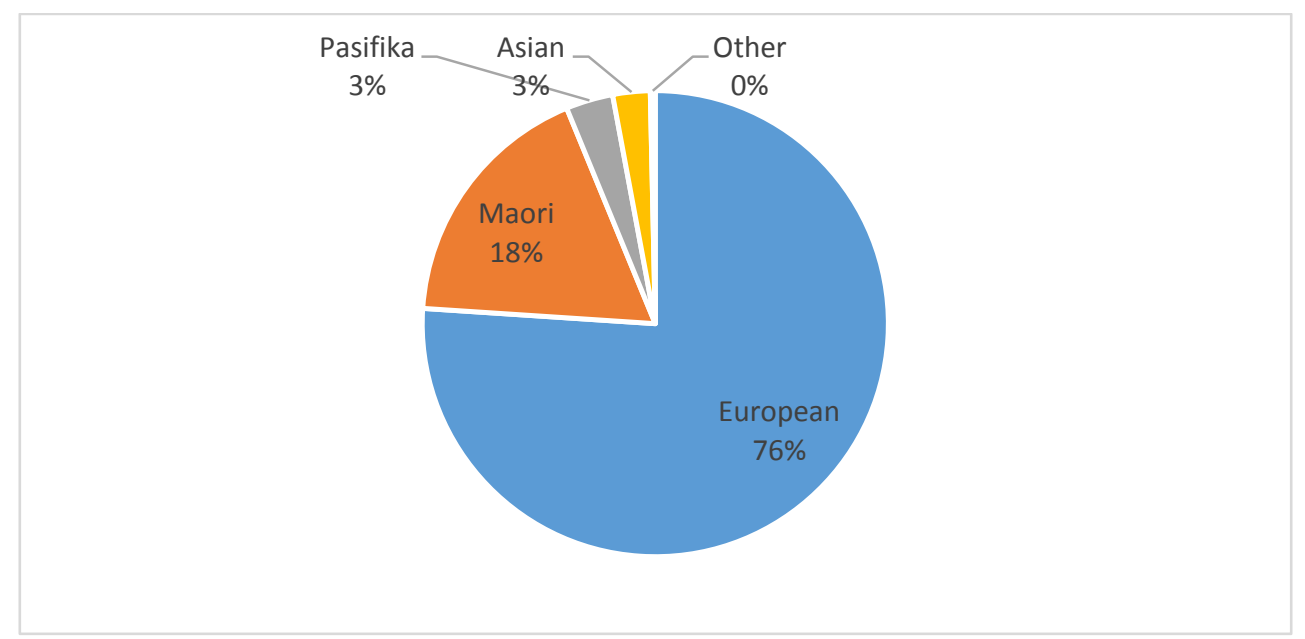

Figure 27: 2006 Census respondents by ethnicity for Hastings and New Plymouth districts

\subsection{Quantitative data results for walkers}

Of the 75 walkers, 18 (24\%) were surveyed in Hastings and 57 (76\%) were surveyed in New Plymouth. The gender split was almost equal, with $49 \%$ of respondents identifying themselves as female and $51 \%$ as male. The 50-59 year olds were the largest group with 16 respondents and the 70 and older were the smallest group with five respondents.

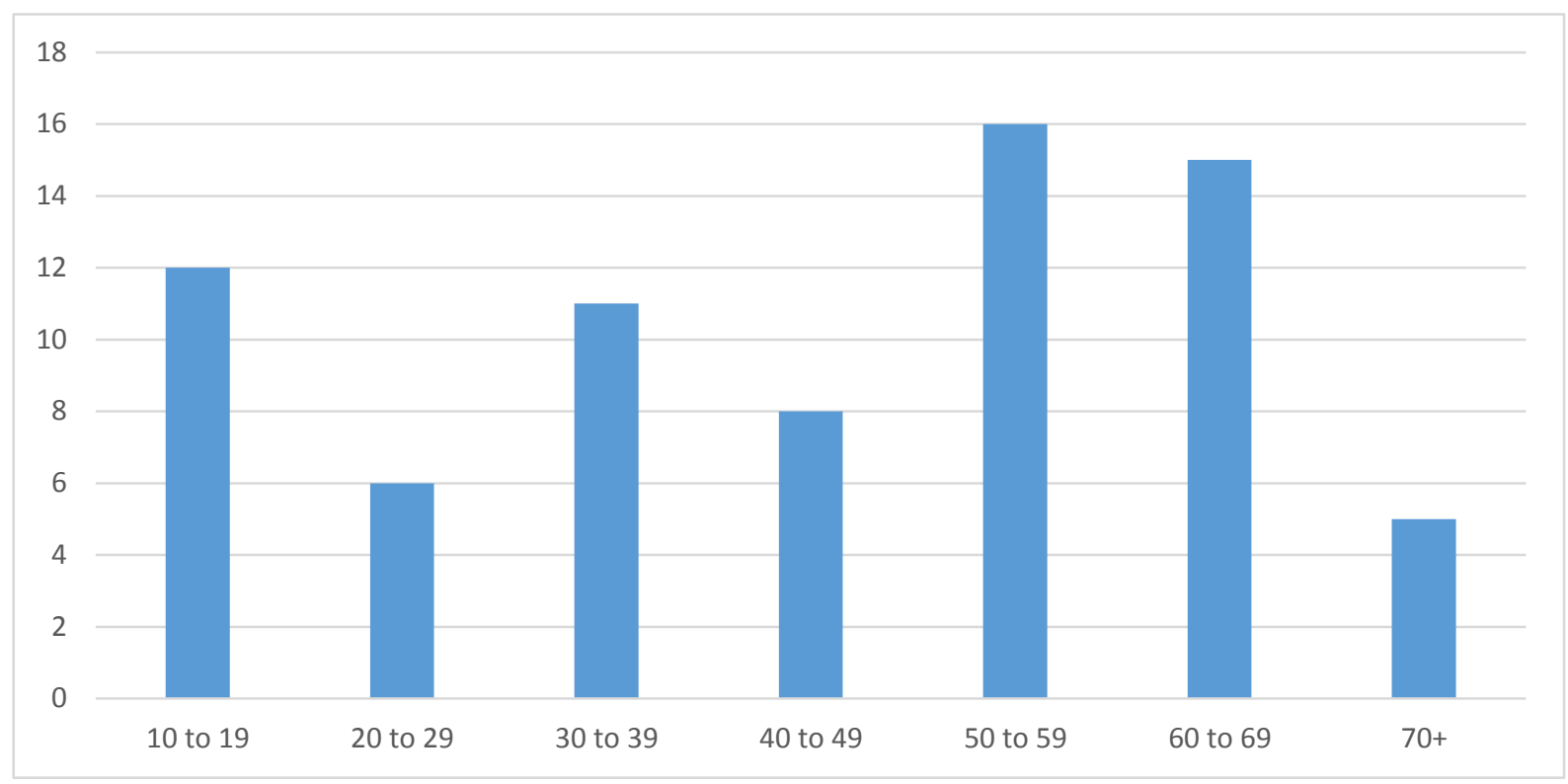

Figure 28: MCP intercept survey walkers by age group, $n=73$ 
At the time of the survey, December 2013, 54 of the 75 walkers (72\%) were aware of iWay or Let's Go. Of the 75 walkers surveyed, 73 (97\%) reported walking on a regular basis (at least once a week for at least ten minutes at a time) in 2013. Of these 73 regular walkers in 2013, 48 (66\%) reported that they were regular walkers in their respective district councils in 2009, and this sample was used to estimate average distance walked in 2009. Nine (12\%) regular walkers in 2013 reported not being regular walkers in 2009, while 14 (19\%) regular walkers in 2013 reported living outside of Hastings or New Plymouth in 2009.

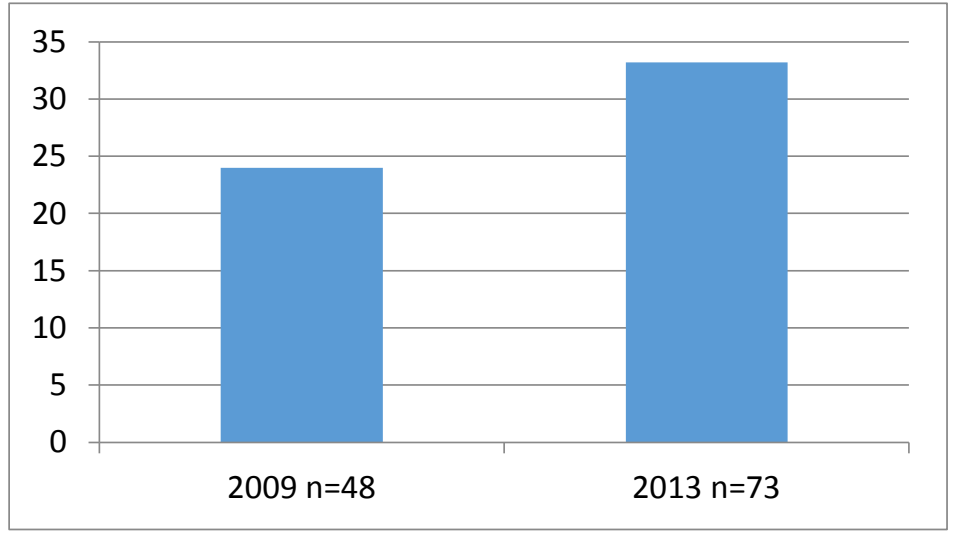

Figure 29: MCP Average distances walked per week (in km)

The 48 respondents who were regular walkers in 2009 and 2013 reported walked an average of $24 \mathrm{~km}$ a week in 2009. In comparison, the 73 regular walkers in 2013 reported walking an average of $33.2 \mathrm{~km}$ a week in 2013 (Figure 29), representing an increase of $38.3 \%$ in average $\mathrm{km}$ walked per week from 2009 to 2013 . When limiting thesample to just those respondents who regularly walked in 2009, these 48 walkers reported walking $33.4 \mathrm{~km}$ a week in 2013 , about $0.6 \%$ more than the collective group. Of the 48 respondents who were regular walkers in 2009 and 2013, 26 (54\%) reported walking more in an average week in 2013 while 18 (38\%) reported walking the same average distance per week and 4. (8\%) reported walking less on average in 2013 (Figure 30). It is worth noting that these aforementioned values do not include the two walkers who reported being regular walkers in 2009 but were not regular walkers in 2013.

All respondents who were regular walkers in 2013 were asked to rate the walking facilities in their district on a scale of 1 (low) to 10 (high) across six criteria: personal safety, surface 
quality, separation from motor vehicles, coverage ${ }^{1}$, enjoyment and positive motorist attitudes towards walkers. Respondents who were regular walkers in 2009 were asked to rate the facilities for 2009 as well.

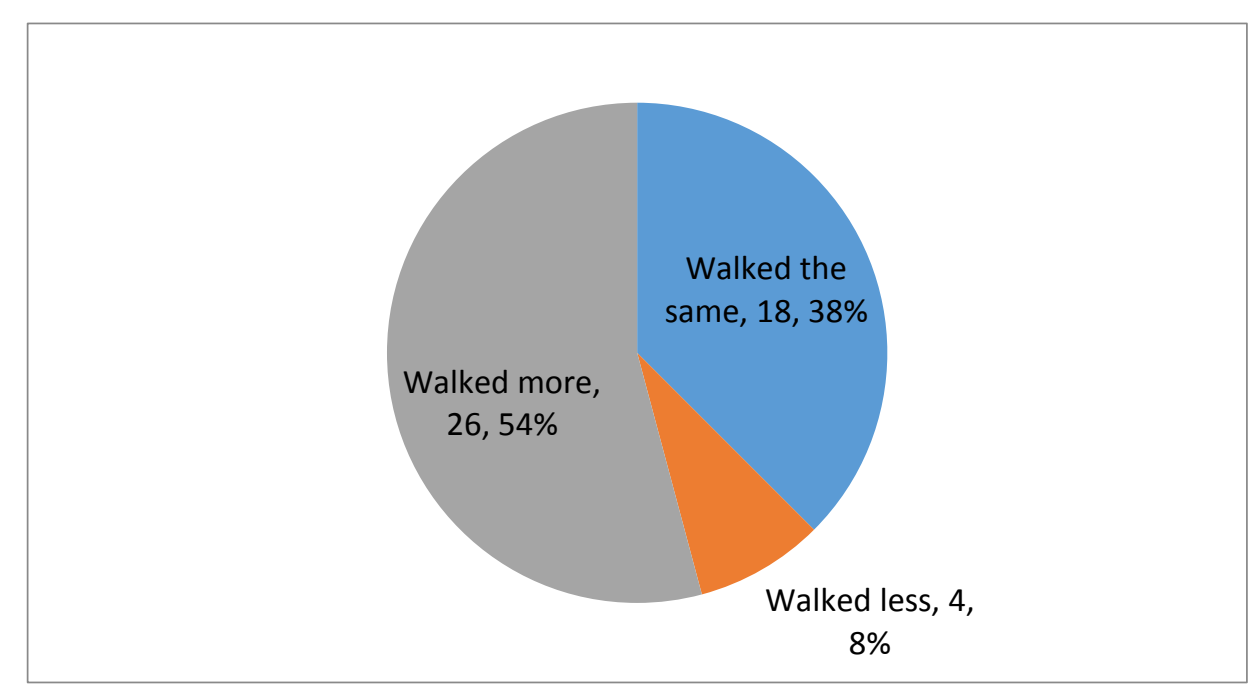

Figure 30: Intercept survey walkers' reported 2013 weekly walking distances compared to 2009, $n=48$

Walkers' average ratings of walking facilities in Hastings and New Plymouth achieved modest gains from 2009 to 2013. When comparing 2009 facility ratings with 2013 ratings by the same walkers, coverage achieved the highest ratings increase, a full point (7.8 to 8.8); the 2013 coverage rating for all 2013 regular walkers is almost identical (8.7).

Ratings for personal safety received the least rating increase from 2009 to 2013, with only a 0.3 increase given by 2013 regular walkers who were also regular walkers in 2009 (0.4 using ratings by all 2013 regular walkers). The 2013 ratings did not vary by more than 0.1 between 2013 regular walkers who were also regular walkers in 2009 and all 2013 regular walkers, with the exception of the 'positive motorist attitudes towards walkers' criterion; for this, the rating for all 2013 regular walkers was 0.2 greater than the rating by the 2009 'baseline' group (Table 24).

\footnotetext{
${ }^{1}$ Coverage was explained, when necessary, as how much of the city is serviced by [cycling or walking] facilities
} 


\begin{tabular}{|c|c|c|c|c|c|c|}
\hline & $\begin{array}{l}\text { Personal } \\
\text { safety }\end{array}$ & $\begin{array}{l}\text { Surface } \\
\text { quality }\end{array}$ & $\begin{array}{l}\text { Separation } \\
\text { from } \\
\text { motor } \\
\text { vehicles }\end{array}$ & 更 & Enjoyment & $\begin{array}{l}\text { Positive } \\
\text { motorist } \\
\text { attitudes } \\
\text { towards } \\
\text { walkers }\end{array}$ \\
\hline $\begin{array}{l}\text { Rating of } 2009 \\
\text { facilities by } \\
\text { regular } 2009 \\
\text { walkers }(\mathrm{n}=48 \text { ) }\end{array}$ & 7.3 & 7.0 & 7.5 & 7.8 & 8.4 & 6.5 \\
\hline $\begin{array}{l}\text { Rating of } 2013 \\
\text { facilities by } \\
\text { walkers who } \\
\text { regularly } \\
\text { walked in } \\
2009(\mathrm{n}=48)\end{array}$ & 7.6 & 7.7 & 8.3 & 8.8 & 9.1 & 6.9 \\
\hline $\begin{array}{l}\text { Rating of } 2013 \\
\text { facilities by } \\
\text { regular } 2013 \\
\text { walkers }(\mathrm{n}=73 \text { ) }\end{array}$ & 7.7 & 7.8 & 8.2 & 8.7 & 9.0 & 7.1 \\
\hline
\end{tabular}

Table 24: MCP walker ratings of facilities between 2009 and 2013

Walkers were also asked to rank the criteria in order of most important (1) to least important (6), as summarised in Table 25. Walkers ranked personal safety as the most important criterion with an average ranking 2.3 , whereas positive motorist attitudes towards walkers received the lowest average ranking (5.0). Both personal safety and enjoyment received more number one rankings ratings than any other ranking (Figure 31). $57 \%$ of respondents ranked positive motorist attitudes towards walkers as the 6 th or least important criterion, while no respondents ranked personal safety as the least important criteria (Figure 32). 


\begin{tabular}{|c|c|c|c|c|c|c|}
\hline & $\begin{array}{l}\text { Personal } \\
\text { safety }\end{array}$ & $\begin{array}{l}\text { Surface } \\
\text { quality }\end{array}$ & $\begin{array}{l}\text { Separation } \\
\text { from motor } \\
\text { vehicles }\end{array}$ & Coverage & Enjoyment & $\begin{array}{l}\text { Positive } \\
\text { motorist } \\
\text { attitudes } \\
\text { towards } \\
\text { walkers }\end{array}$ \\
\hline mean & 2.3 & 3.6 & 4.0 & 3.5 & 2.7 & 5.0 \\
\hline median & 2 & 3 & 4 & 4 & 2 & 6 \\
\hline mode & 1 & 3 & 5 & 4 & 1 & 6 \\
\hline $\mathrm{n}$ & 73 & 72 & 73 & 72 & 72 & 72 \\
\hline
\end{tabular}

Table 25: Walkers' ranking of criteria in Hastings and New Plymouth (1=most important, $6=$ least)

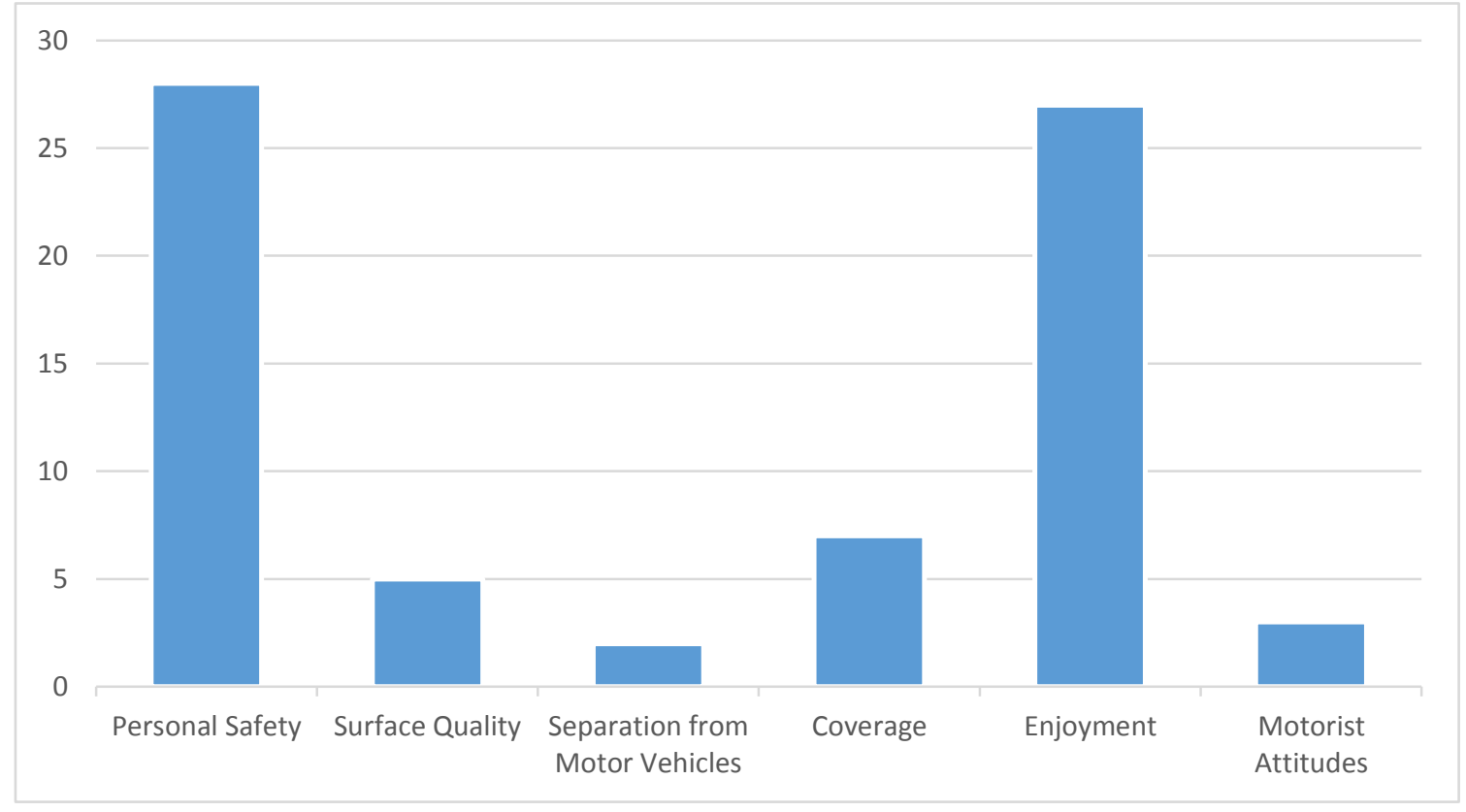

Figure 31: Frequency of \#1 rankings for walkers per criteria 2013, $n=72$ 


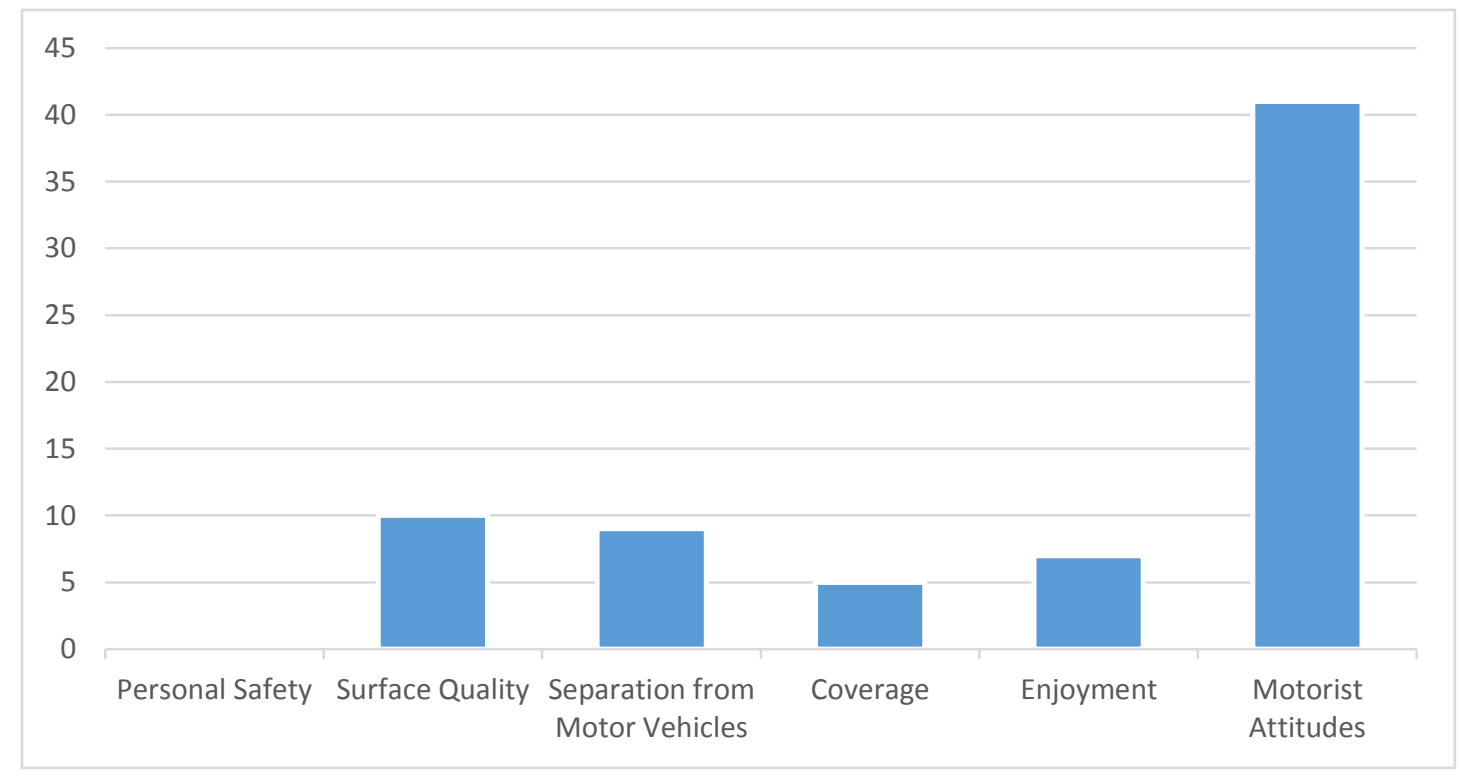

Figure 32: Frequency of \#6 rankings for walkers per criteria 2013, $n=72$

Walkers were also asked to place a per year value on walking facilities in Hastings and

New Plymouth in increments of $\$ 0-\$ 50, \$ 51-\$ 100, \$ 101-\$ 200, \$ 201-\$ 500$, and $>\$ 500$.

Out of 47 total respondents, 20 (43\%) valued the walking facilities at greater than $\$ 500$ per year, the most frequent of any value. A value of $\$ 201-\$ 500$ was second with 15 responses (32\%), $\$ 101$ to $\$ 200$ was third with nine responses (19\%), $\$ 51$ to $\$ 100$ was fourth with three $(6 \%)$ responses, and no respondents chose a value of $\$ 50$ or less (Figure 33).

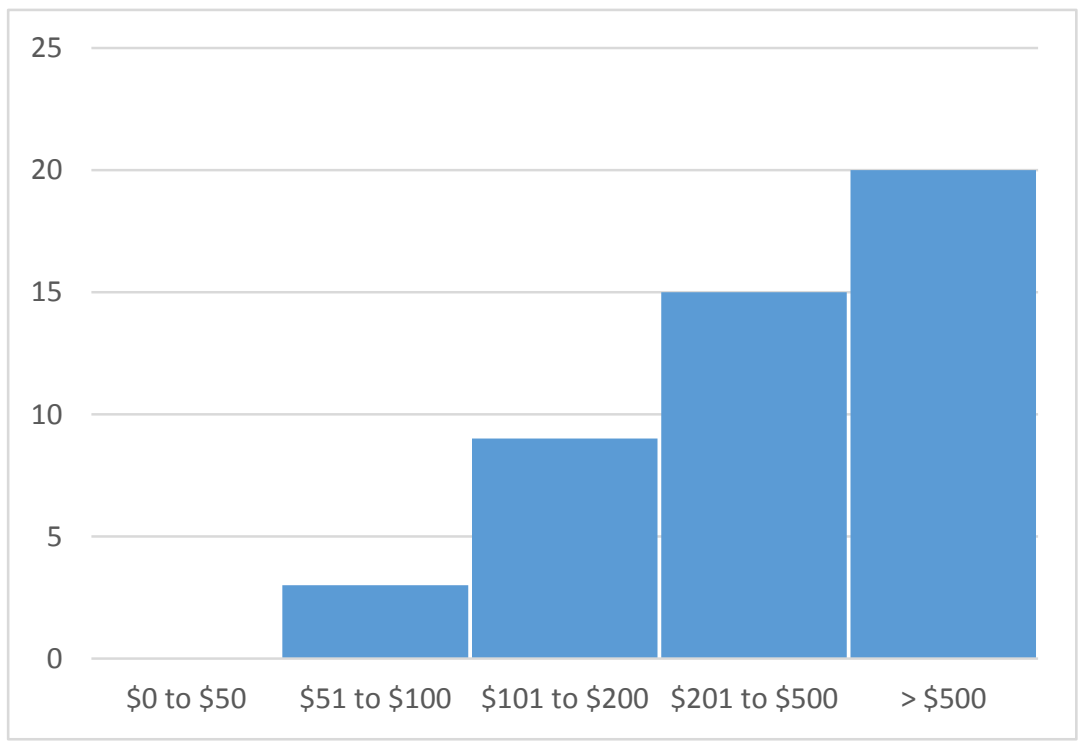

Figure 33: Value per year (in \$) placed by surveyed walkers on walking facilities in Hastings and New Plymouth, $n=47$ 


\subsection{Quantitative data results for cyclists}

Of the 90 cyclists surveyed, 37 (41\%) were surveyed in New Plymouth while 53 (59\%) were surveyed in Hastings. $71 \%$ of respondents identified themselves as male compared to $29 \%$ for female. The distribution of ages is shown in Figure 34. 84\% of cyclists surveyed were aware of $i$ Way or Let's Go.

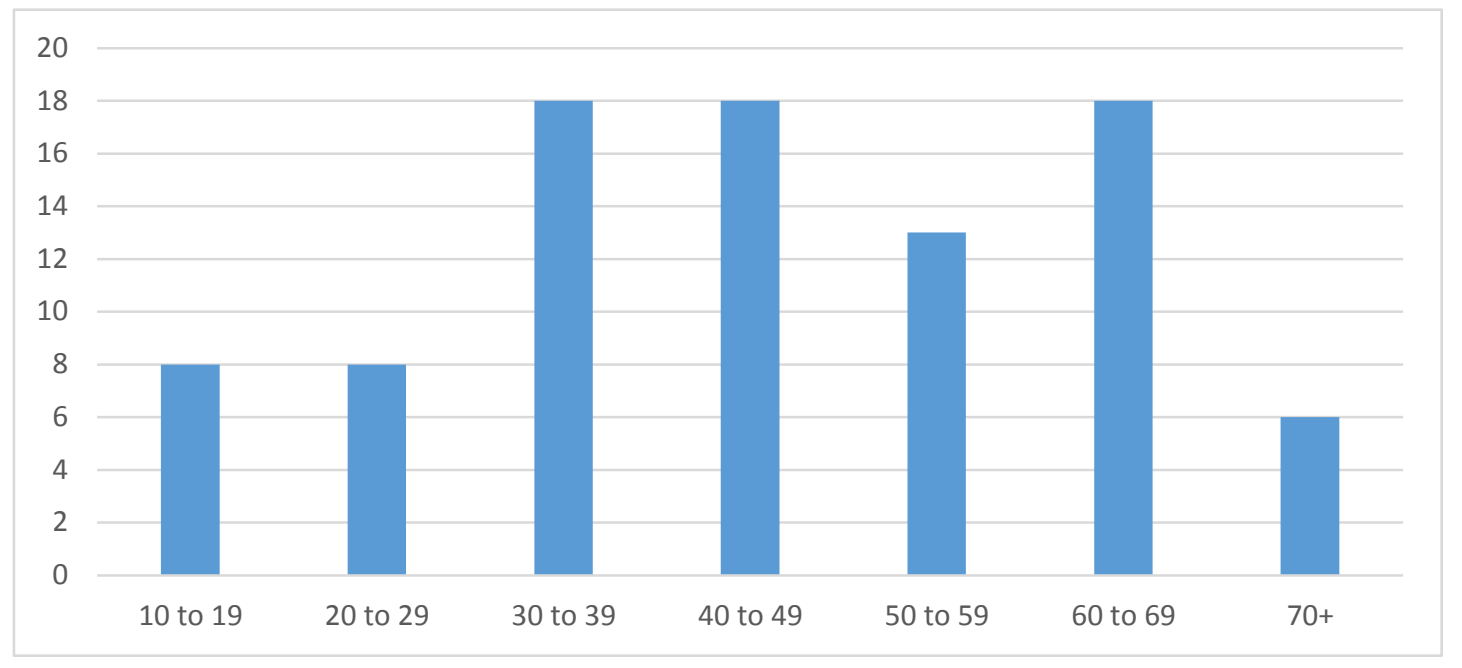

Figure 34: MCP intercept survey cyclists by age group, $n=89$

Of 90 cyclists surveyed, $40 \%$ reported regularly cycling in Hastings or New Plymouth in 2009. From the remaining $60 \%$ of cyclists, $29 \%$ stated that they were not regular cyclists in 2009 (and were living in Hastings or New Plymouth) and 31\% stated that they were not living in Hastings or New Plymouth in 2009 (Figure 35).

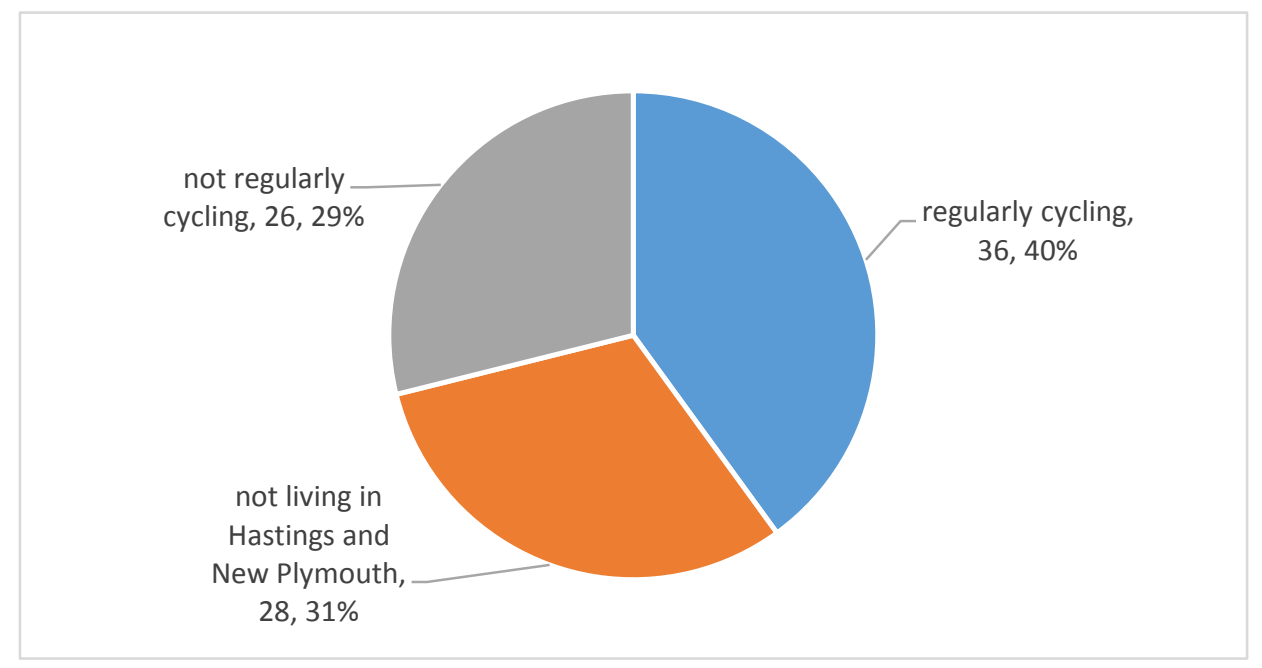

Figure 35: Respondents regularly cycling in 2013, in 2009 were: (n=90) 
The 36 cyclists who were regular cyclists in 2009 and 2013 reported cycling on average $87.8 \mathrm{~km}$ per week in 2009. In 2013, the 90 regular cyclists reported cycling on average $74.3 \mathrm{~km}$ per week, representing a $15.4 \%$ decrease in average $\mathrm{km}$ cycled per week per cyclist (Figure 36). This figure changes significantly however, when seen in terms of those who were also regular cyclists in 2009. Cyclists who cycled regularly in both 2009 and 2013 reported cycling on average $101.4 \mathrm{~km}$ per week in 2013 , showing a $15.5 \%$ increase from 2009. Cyclists who were cycling regularly in 2013 but not 2009 reported cycling on average $56.2 \mathrm{~km}$ a week, or $44.6 \%$ less than those who were regular cyclists in both periods (Figure 37).

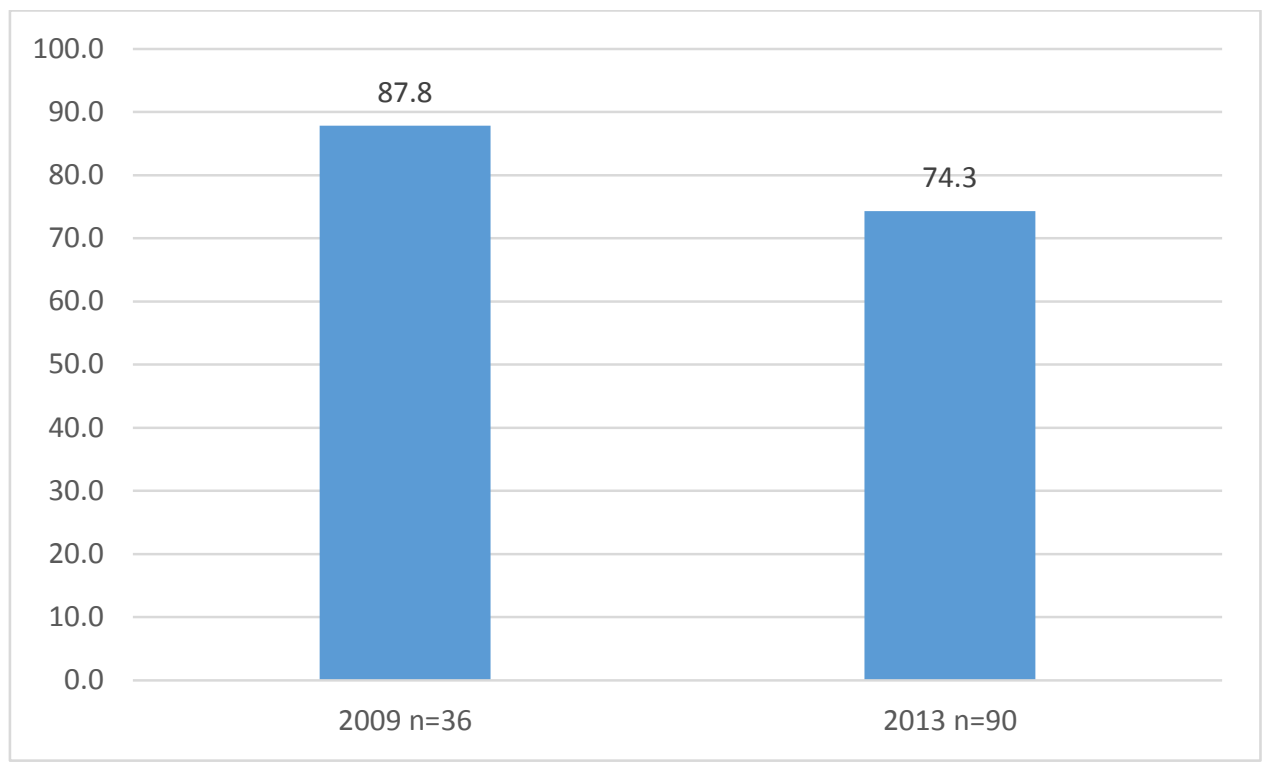

Figure 36: Mean distance cycled in km per cyclist by year

Of the 36 cyclists who reported regularly cycling in both 2009 and 2013, 22 (61\%) claim they cycled on average greater distances per week than in 2009. Eight cyclists (22\%) reported cycling on average the same distance per week; and six (17\%) stated they cycled less on average per week in 2013 compared to 2009 (Figure 38). 


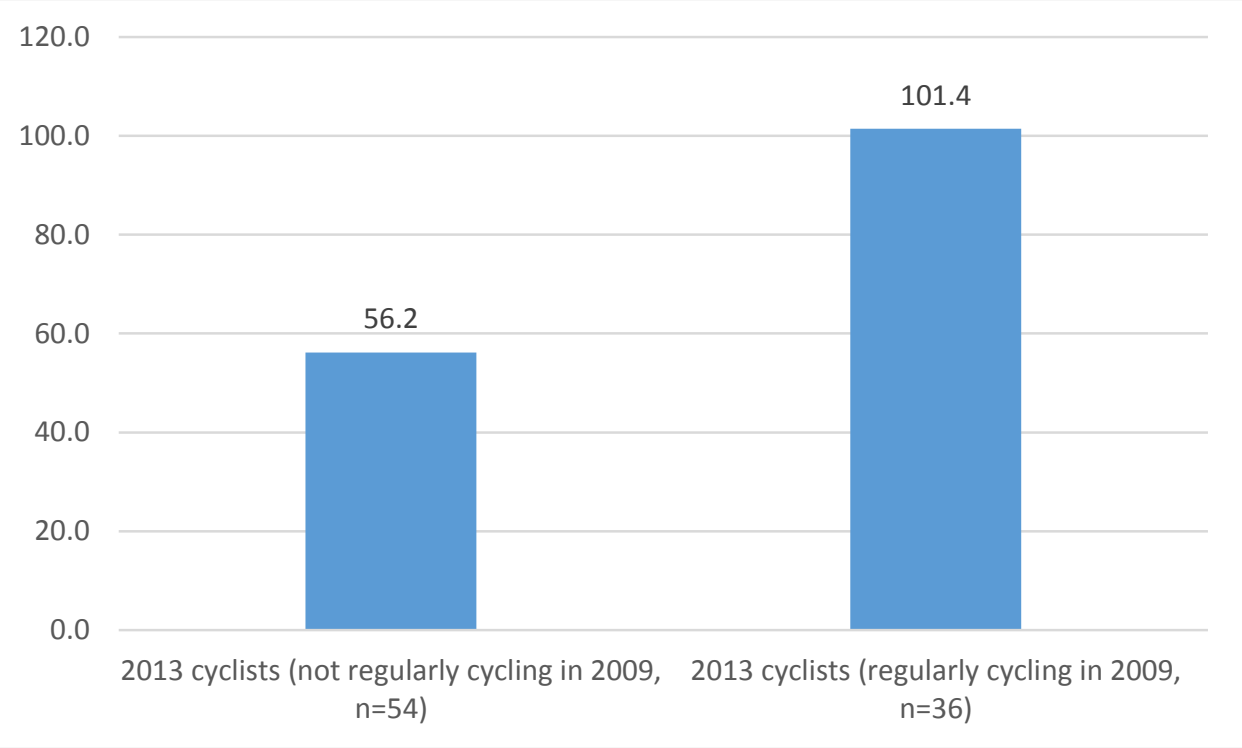

Figure 37: Mean 2013 weekly cycling distances in km

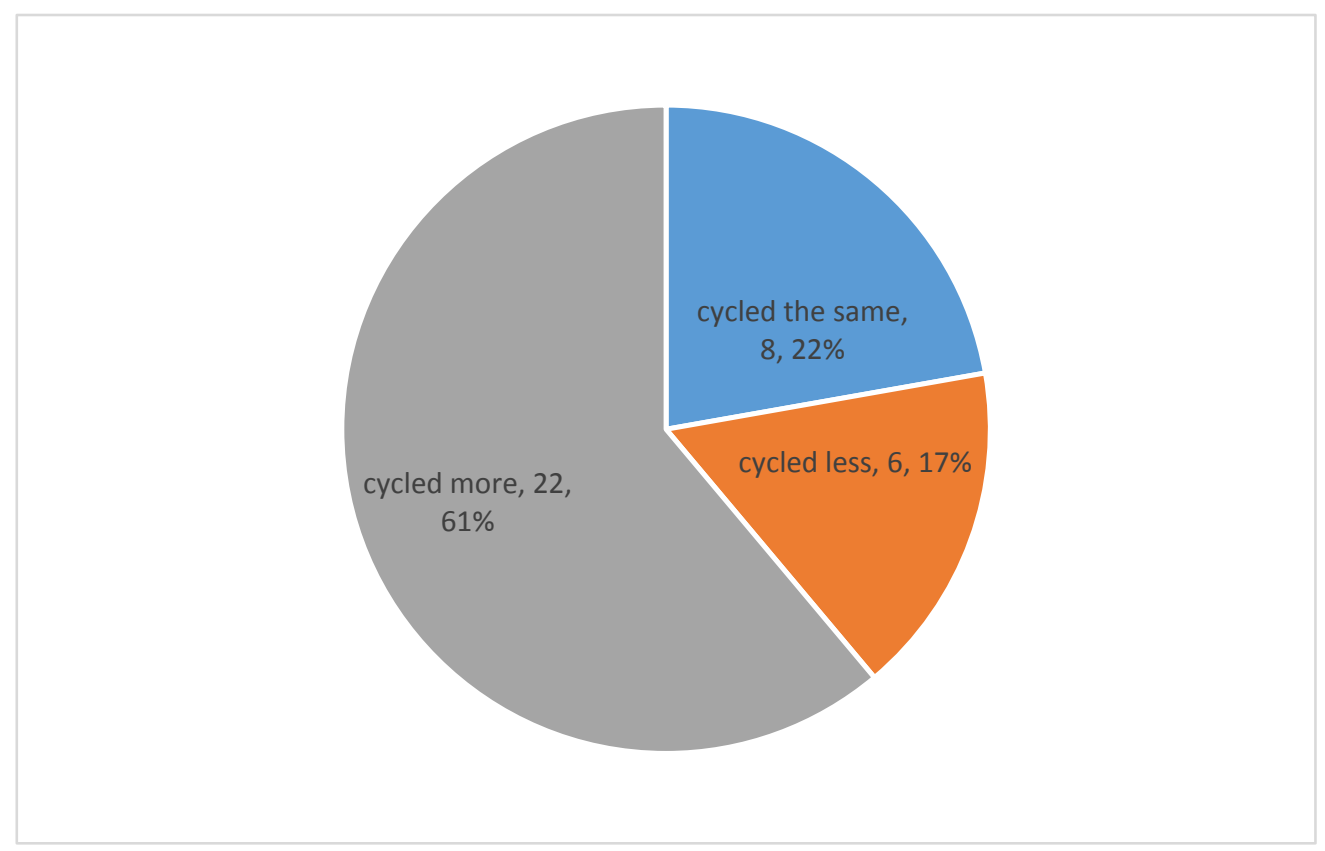

Figure 38: Companing average weekly distances cycled for cyclists who regularly cycled in both 2009 and 2013. In 2013 they: $(n=36)$ 
Like the walkers with walking facilities, cyclists were asked to rate cycling facilities both before the intervention $(2009, n=36)$ and after the intervention $(2013, n=90)$. The criteria were the same as for walkers, but applied within a cycling context. There were significant increases in cyclists' ratings for all criteria. When comparing 2009 and 2013 ratings for cyclists who regularly cycled in both periods $(n=36)$, separation from motor vehicles increased the most, by 3.4 points. Within this same group of cyclists, coverage increased the second most by 2.8 points, followed by surface quality by 2.6 points, personal safety by 2.4 points, enjoyment by 1.9 points, and finally positive motorist attitudes which increased by 0.9 points. When comparing 2013 regular cyclists who were also regularly cycling in 2009 to those who were not, the ratings varied by 0.1 points for personal safety, surface quality, separation from motor vehicles, and varied by 0.4 points for coverage and positive motorist attitudes towards cyclists, with both groups of cyclists rating enjoyment the same at 8.9 out of 10 (Table 26).

\begin{tabular}{|c|c|c|c|c|c|c|}
\hline & $\begin{array}{l}\text { Personal } \\
\text { safety }\end{array}$ & $\begin{array}{l}\text { Surface } \\
\text { quality }\end{array}$ & $\begin{array}{l}\text { Separation } \\
\text { from } \\
\text { motor } \\
\text { vehicles }\end{array}$ & Coverage & Enjoyment & $\begin{array}{l}\text { Positive } \\
\text { motorist } \\
\text { attitudes } \\
\text { towards } \\
\text { cyclists }\end{array}$ \\
\hline $\begin{array}{l}\text { Rating of } \\
2009 \text { facilities } \\
\text { by regular } \\
2009 \text { cyclists } \\
(n=36)\end{array}$ & 4.9 & 5.0 & 3.8 & 5.7 & 7.0 & 4.9 \\
\hline $\begin{array}{l}\text { Rating of } \\
2013 \text { facilities } \\
\text { by cyclists } \\
\text { who regularly } \\
\text { walked in } \\
2009(n=36)\end{array}$ & 7.4 & 7.6 & 7.2 & 8.4 & 8.9 & 5.8 \\
\hline $\begin{array}{l}\text { Rating of } \\
2013 \text { facilities } \\
\text { by regular } \\
2013 \text { cyclists } \\
(n=90)\end{array}$ & 7.5 & 7.5 & 7.3 & 8.0 & 8.9 & 6.2 \\
\hline
\end{tabular}


All 2013 regular cyclists were asked to rank the criteria in order of the most important (1) to the least important (6). Separation from motor vehicles was assessed as the most important with an average ranking of 2.4, with personal safety receiving a ranking of 3.0. Surface quality was ranked the third most important with an average ranking of 3.6, followed by coverage fourth at 3.7, enjoyment fifth at 4.2, leaving positive motorist attitudes towards cyclists of least importance at 4.2 (Table 27).

\begin{tabular}{|c|c|c|c|c|c|c|}
\hline & $\begin{array}{l}\text { Personal } \\
\text { Safety }\end{array}$ & $\begin{array}{l}\text { Surface } \\
\text { Quality }\end{array}$ & $\begin{array}{l}\text { Separation } \\
\text { from } \\
\text { motor } \\
\text { vehicles }\end{array}$ & Coverage & Enjoyment & $\begin{array}{l}\text { Positive motorist } \\
\text { attitudes towards } \\
\text { cyclists }\end{array}$ \\
\hline average & 3.0 & 3.6 & 2.4 & 3.7 & 4.1 & 4.2 \\
\hline median & 3 & 4 & 2 & 4 & 4 & 5 \\
\hline mode & 1 & 3 & 1 & 4 & 6 & 6 \\
\hline $\mathrm{n}$ & 90 & 90 & 90 & 90 & 90 & 90 \\
\hline
\end{tabular}

Table 27: Cyclists' 2013 rankings of criteria in Hastings and New Plymouth (1=most important; 6=least)

Separation from motor vehicles, a specific aspect of both safety and comfort, was ranked number one 37 times (41\%), even more frequently than generalised personal safety, ranked number one 30 times (33\%), as seen in Figure 39. In comparison, separation from motor vehicles was ranked number six five times $(5 \%)$ and personal safety was ranked number six 10 times (11\%), as seen in Figure 40. 25.6\% of cyclists put separation of motor vehicles and personal safety as a combined ranked of number one and two (and vice versa). 


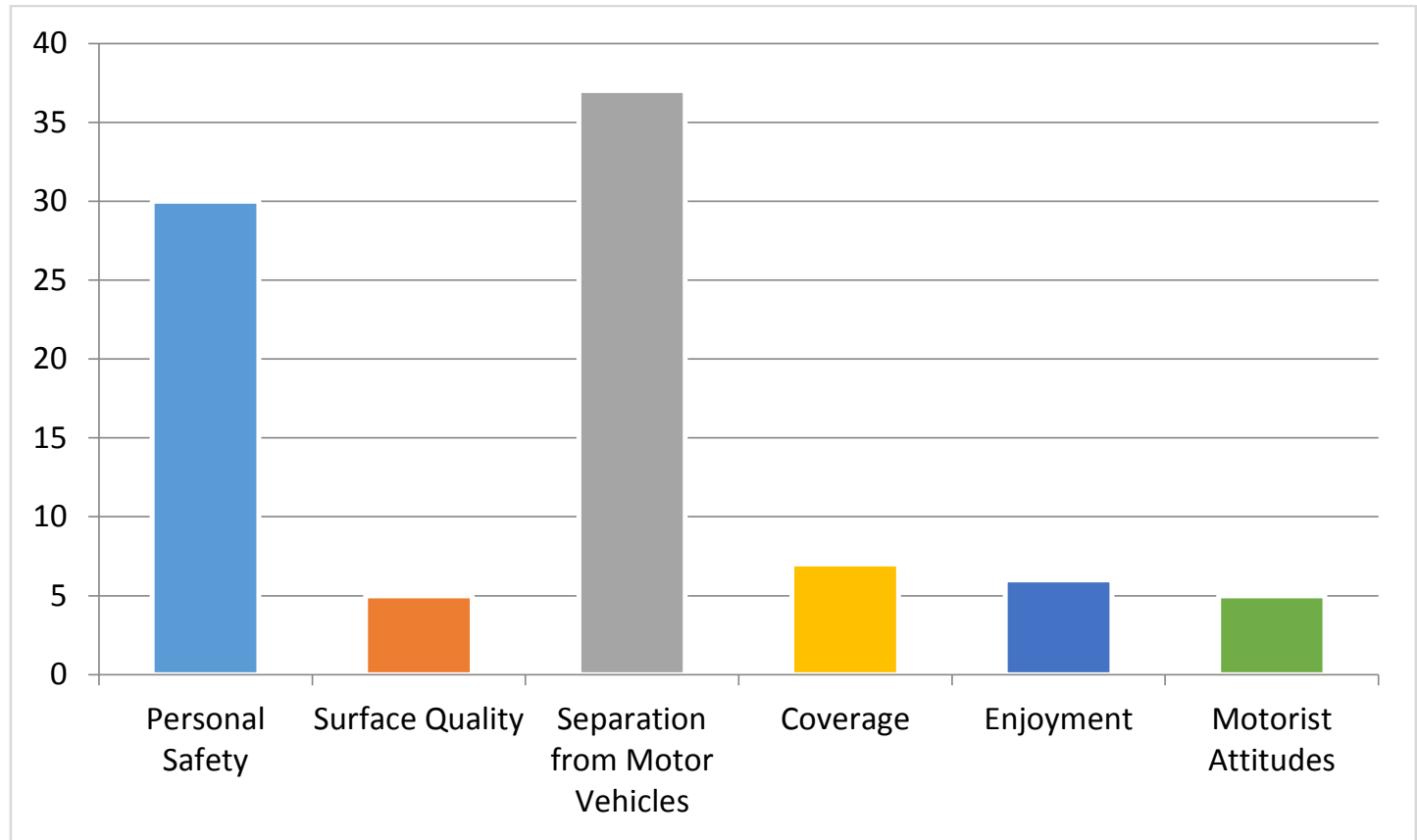

Figure 39: Frequency of \#1 rankings for cyclists per criteria 2013, $n=90$

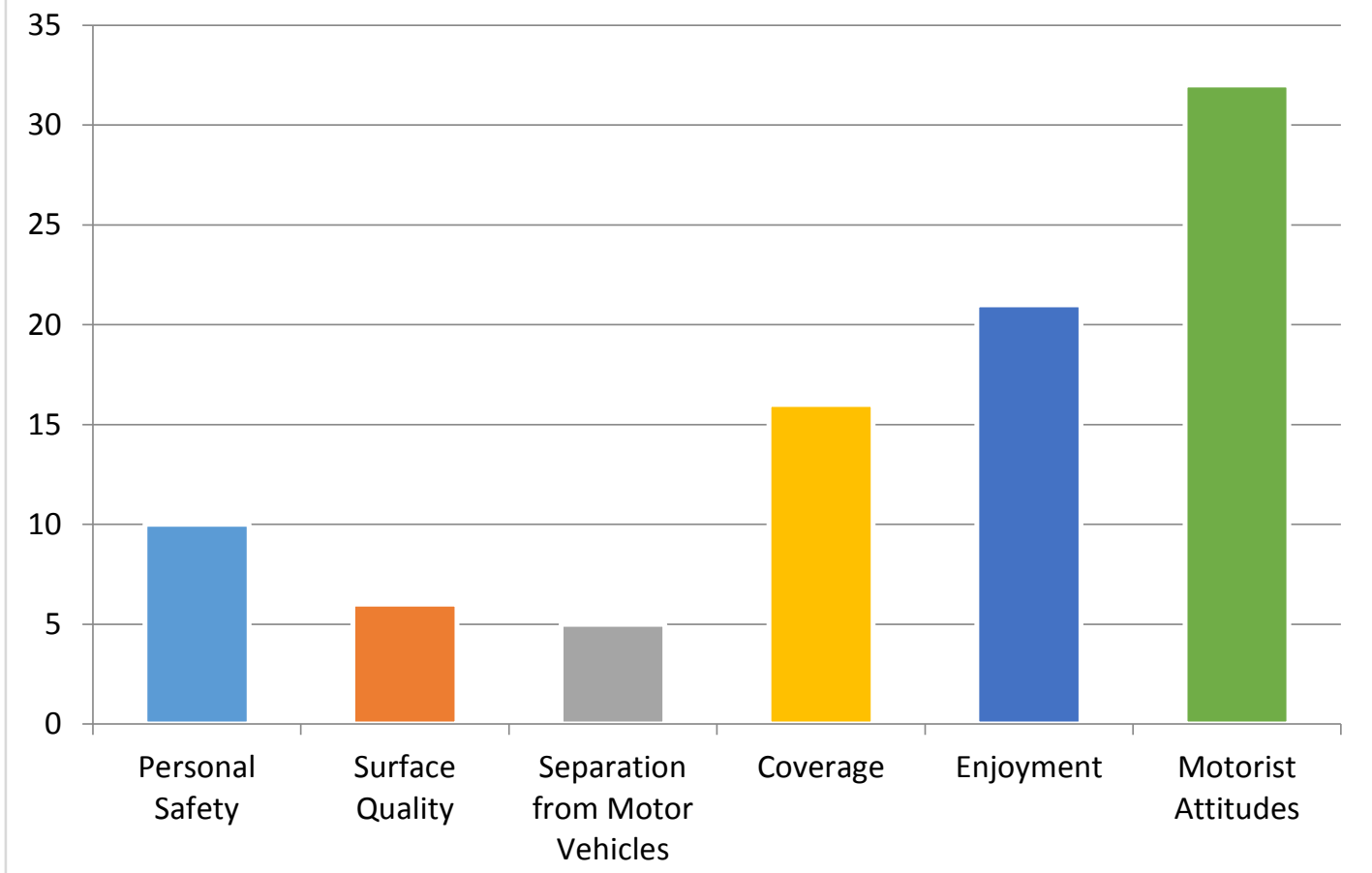

Figure 40: Frequency of \#6 rankings for cyclists per criteria 2013, $n=90$ 
In terms of value per year cyclists place on their cycling facilities in Hastings and New Plymouth, $58 \%$ of cyclists stated they value their cycling facilities by more than $\$ 500$ a year. $27 \%$ of cyclists valued their cycling facilities at $\$ 200-\$ 500$ a year, with $12 \%$ of cyclists valuing them at $\$ 101-\$ 200$ a year, 3\% valuing them at $\$ 51$ to $\$ 100$ a year, and no cyclists valuing them at $\$ 50$ or less a year (Figure 41 ).

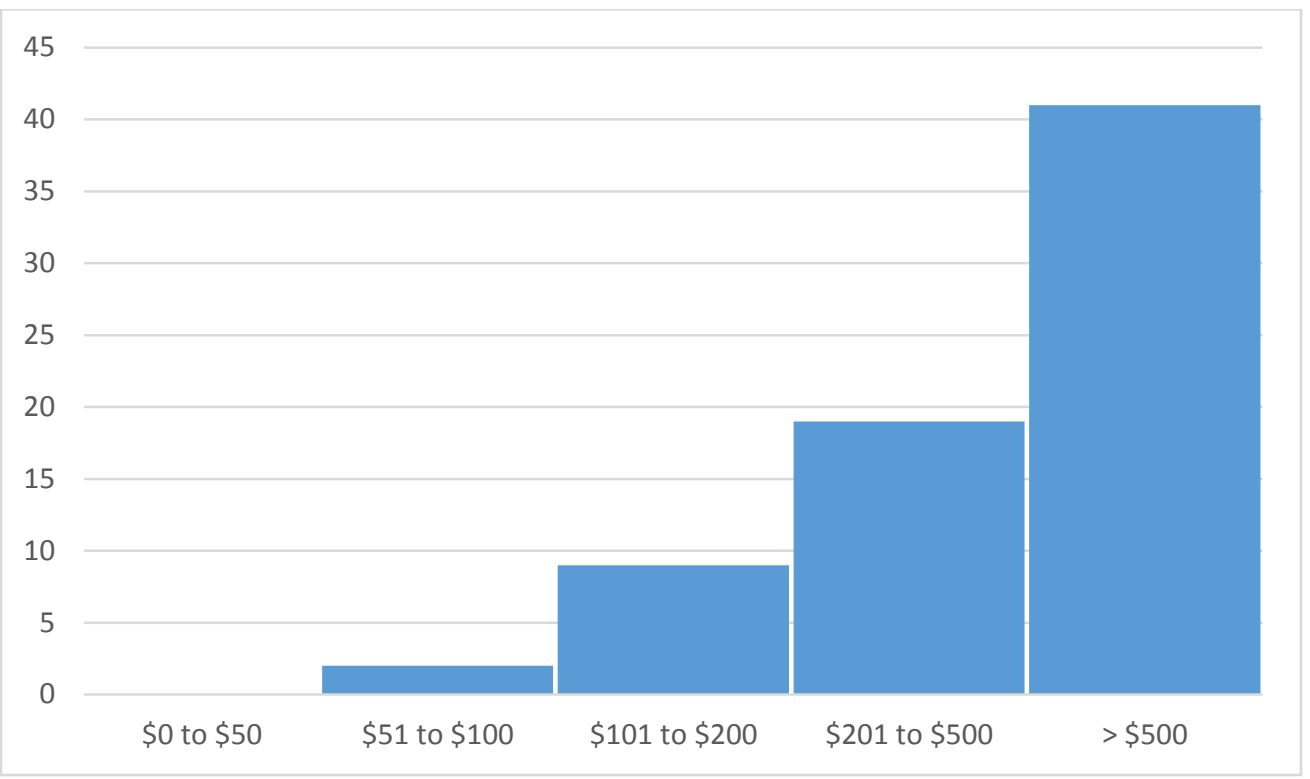

Figure 41: Value per year (in) placed by surveyed cyclists on cycling facilities in Hastings and New Plymouth, n=71

\subsection{Qualitative data results for walkers and cyclists}

In addition to collecting quantitative data from walkers and cyclists, the intercept survey also gathered a substantial amount of qualitative data from users on the improved walking and cycling infrastructure. Questions 13 to 16 in the intercept survey were semistructured in nature, allowing the survey to capture a large array of user feedback. These question focussed on how the MCP has impacted users' lifestyles, transportation habits and community dynamics. Several users willingly (and often enthusiastically) provided commentary that was not necessarily relevant to the questions asked, but often gave important insight into the issue of walking and cycling in Hastings and New Plymouth. This unsolicited commentary was recorded and some of it is summarised in this chapter. 
When these qualitative data were analysed, responses that were commonly given were generalised and reported. In some cases, responses provided for one of the questions were also relevant to other questions in the survey, and sometime these responses were included in the analysis of the other question. Examples of this are included in the question specific conditions analysed below. When users provided insightful and unique responses, these responses were summarised (or cited when quotations marks are present) and put into tables for each question.

\subsubsection{Intercept survey question \#13}

“13) In your own words, briefly describe how the $i$ Way programme (improvements in walking/cycling infrastructure and promotion) has impacted your lifestyle, if at all."

The six most common responses to this lifestyle question were coded and tallied below. In some cases, the responses for "walks/cycles as a result of $i$ Way, Let's Go" were taken from responses to other questions or from unsolicited commentary. All other common responses were tallied directly from participants' responses to this question.

44 users reported cycling more and 17 users reported walking more, while 36 users reported no significant change in lifestyle. A small but notable number of users (6) reported driving less, living a healthier/fitter lifestyle (8), and 7 users claimed they now walk or cycle as a result of the $i$ Way and Let's Go interventions (Figure 42). As this question has a semi-structured format, responses represent participants' chosen responses, and not necessarily all aspects of, or the reality of, their lifestyle change. Thus, the observation can be made that while 8 users explicitly reported being healthier/fitter, it can be expected that there are a greater number who are living healthier/fitter lifestyles who did not express that response. This is true for all responses to questions 13 to 16 of the intercept survey. 


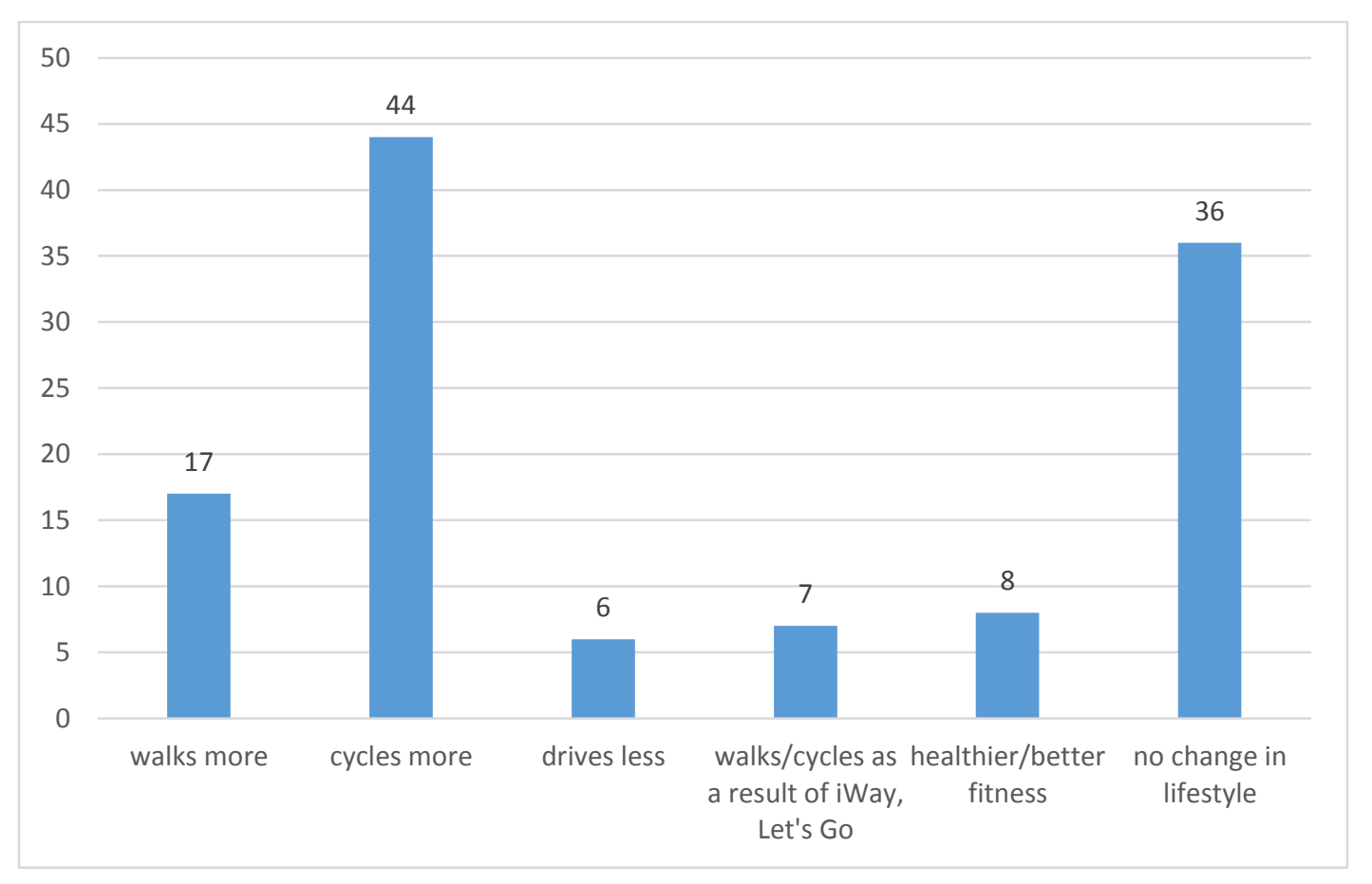

Figure 42: Six most common responses to question \#13 (125 users responding)

For question \#13, eight users provided insightful individual comment. These responses are summarised in Table 28.

\begin{tabular}{|l|l|}
\hline Survey \# & Summarised responses \\
\hline $3 \mathrm{NP}$ & Eats more and sleeps better (works nightshifts) \\
\hline $7 \mathrm{NP}$ & Still regularly walks at age 85 \\
\hline $15 \mathrm{NP}$ & Wasier to walk with pushchairs \\
\hline $118 \mathrm{H}$ & Better places to run \\
\hline $147 \mathrm{H}$ & Is now more comfortable taking his kids for a bicycle ride \\
\hline $148 \mathrm{H}$ & Greater opportunity and peace of mind to cycle in a safe environment \\
\hline $153 \mathrm{H}$ & Does less cycling on road, now rides more on off-road pathways \\
\hline $159 \mathrm{H}$ & \\
\hline
\end{tabular}




\subsubsection{Intercept survey question \#14}

"14) In your own words, briefly describe how the improved walking and cycling infrastructure built in the past three years has changed your transportation habits, it at all.”

This question was focused on transportation rather than, as with Q 13, lifestyle. The 9 responses here were chosen as expected responses to the question. In some cases, users reported a change in transportation habit in question \#13, but not in question \#14. In these cases, these responses from question \#13 were included in the analysis of question \#14. This was only done when the response given in question \#13 did not conflict with a response provided in \#14. For example, if a user responded "cycles more" in question \#13 but responded "no change" in question \#14, the "cycles more" response was not included in the analysis of question \#14.

54 users reported cycling more as a result of the intervention, with another 25 reporting they are walking more. A significant number of users (23) claim to be driving less as result of the intervention, with four driving more, while 49 users claim that their transportation habits have not changed in the past three years as a result of the intervention (Figure 43).

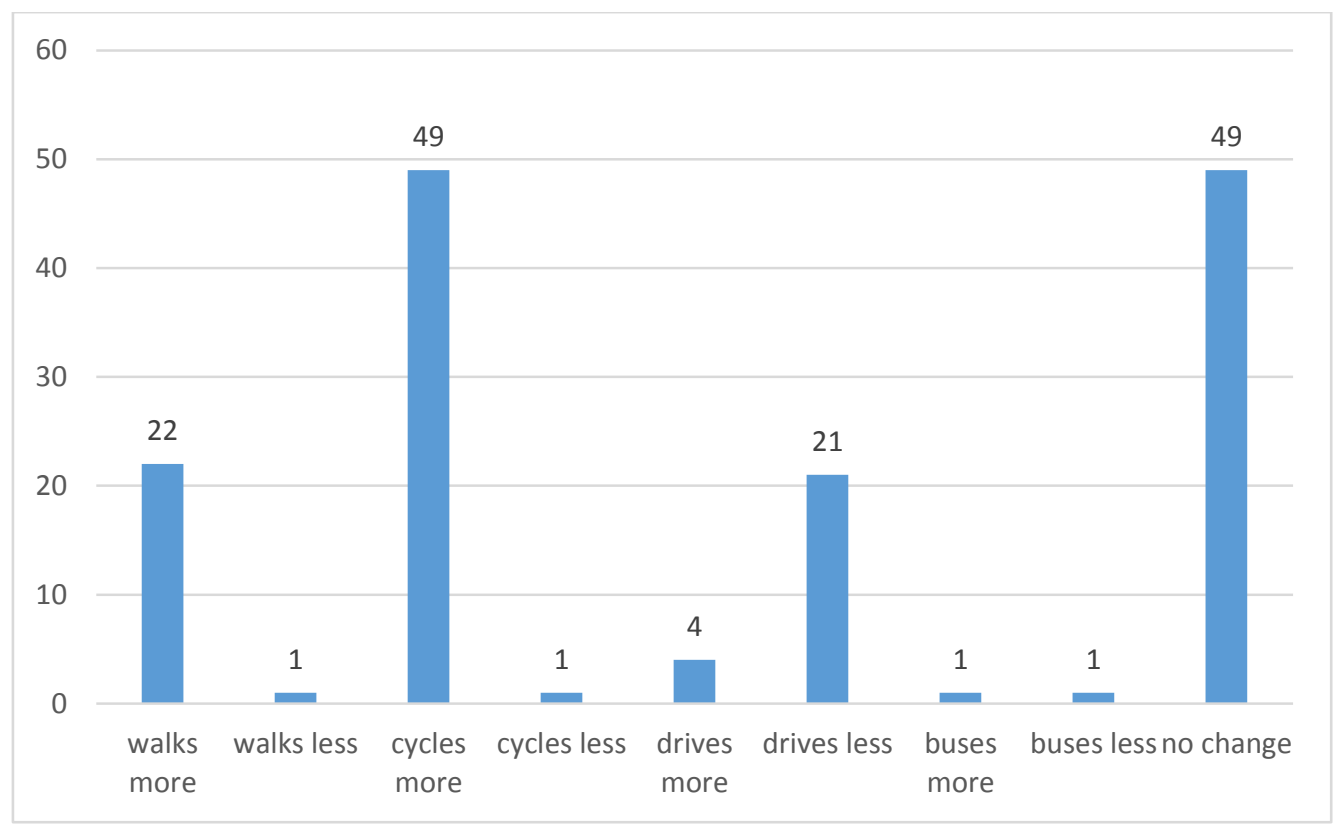

Figure 43: Responses to survey question \#14 (120 users responding) 
Table 29 provides several unique and insightful responses to question \#14.

\begin{tabular}{|l|l|}
\hline Survey \# & Summarised responses \\
\hline $44 \mathrm{NP}$ & Is now a one car family \\
\hline $52 \mathrm{NP}$ & Family only uses one car now \\
\hline $54 \mathrm{NP}$ & Former runner, now walks due to a recent stroke \\
\hline $55 \mathrm{NP}$ & Gave up car \\
\hline $81 \mathrm{NP}$ & Works from home, now has greater incentive to take walking breaks \\
\hline $106 \mathrm{H}$ & Now cycles "a hell of a lot more" \\
\hline $119 \mathrm{H}$ & Cycles with family more \\
\hline
\end{tabular}

Table 29: Summarised responses to Question \#14

\subsubsection{Intercept survey question \#15}

“15) In your own words, briefly describe how the improved walking and cycling infrastructure built in the past three years is likely to affect your transportation habits in the next five years, if at all.”

This question was prospective, focusing on the next five years. The responses here reflect the expected responses also used in question \#14. Slightly more than half of the respondents (66) claim that the improved infrastructure will not affect their transportation habits in the next five years. This may reflect that some respondents' use of the infrastructure may have already plateaued. A significant number of respondents (30) claim they will cycle more, while a modest number of respondents report they will walk more (11) and drive less (10) within the next five years as a result of the improved walking and cycling infrastructure. Six respondents also report they plan on driving more in the next five years (Figure 44). 


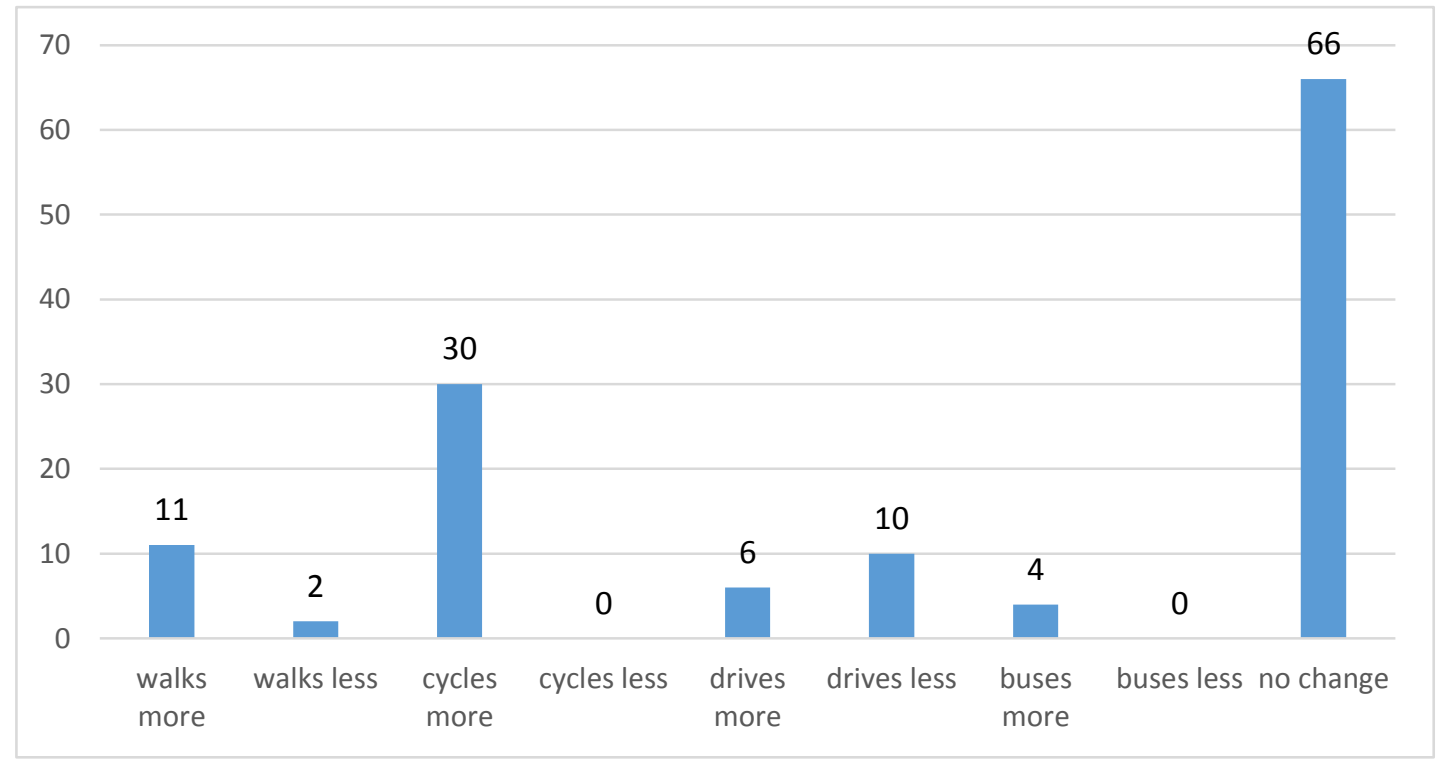

Figure 44: Responses to survey question \#15 (119 users responding)

Table 30 provides some summarised responses that provide additional insight for question \#15.

\begin{tabular}{|l|l|}
\hline Survey \# & Summarised response \\
\hline $7 \mathrm{NP}$ & Will keep on walking (85 years old) \\
\hline $9 \mathrm{NP}$ & Won't need a car \\
\hline $18 \mathrm{NP}$ & Plans on getting groceries by bicycle \\
\hline $112 \mathrm{H}$ & Will likely lose driver's license at next renewal, hopes to continue biking (80 years old) \\
\hline $133 \mathrm{H}$ & Will drive more after getting driver's license (teenager) \\
\hline
\end{tabular}

Table 30: Summarised responses to Question \#15 


\subsubsection{Intercept survey question \#16}

“16) In your own words, briefly describe any way in which the improved walking and cycling infrastructure built in the last three years has affected your community within the Hastings/New Plymouth District.”

Figure 45 shows the 8 most common responses to question \#16. It is important to note that several respondents reported that people are more "active". I have included this response for both "more people walking" and "more people cycling", as they are by and large the largest users of the improved facilities. There were also a large number of respondents differentiating between whether people were walking or cycling more. The "more social communities" response includes general comments about greater communication between users, recognizing friends and other regular users, and any comments suggesting a more cohesive community. Any comment suggesting walking or cycling routes/facilities were safer were counted under "safer walking and cycling routes". Improved accessibility included any comment suggesting that it was easier for a particular group to access walking and cycling facilities, notably vulnerable groups such as children, the elderly and the disabled, or even comments simply suggesting ease of reaching any destination. "Increased tourism" included any comment about seeing more out of town or overseas visitors using the improved walking and cycling facilities. Any comment related to the collective community health and fitness was counted under "healthier/more active communities”. Any comment that specifically mentioned benefits to youth or school children was placed under "beneficial to youth", though it is worth noticing some of these will overlap with the "improved accessibility" response.

Respondents by and large noted that people in their local communities are more active as a result of the intervention, with references to more people walking or cycling at more than 60 per mode. 36 respondents stated that the intervention has improved accessibility for all users, with 13 respondents explicitly reporting that the intervention has largely benefitted school children and/or youth. 26 respondents noted the interventions provision of safer walking and cycling routes, while 21 respondents noted that the intervention has allowed for more social communities. 17 respondents reported that the intervention has created healthier and more active communities, and 11 respondents have claimed that the intervention has increased tourism to their district council. 


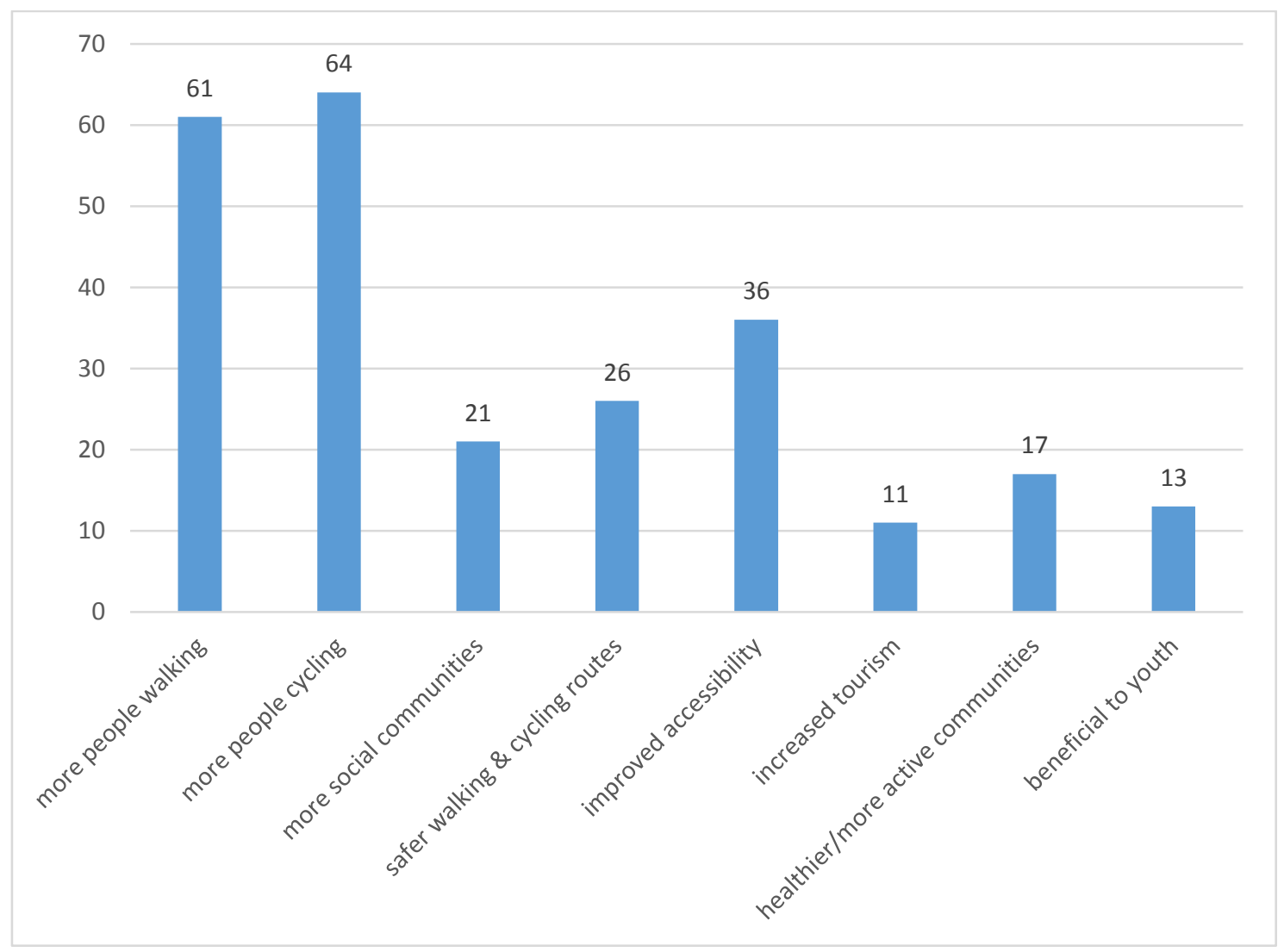

Figure 45: 8 common responses to survey question \#16 (144 users responding)

There were a large number of other responses that are worth mentioning. They are listed in Table 31 .

\begin{tabular}{|l|l|}
\hline Survey \# & Summarised responses \\
\hline $3 \mathrm{NP}$ & People walking at all times of the day \\
\hline $12 \mathrm{NP}$ & More kids walking and cycling to school \\
\hline $18 \mathrm{NP}$ & Often recognises friends and other regular users \\
\hline $20 \mathrm{NP}$ & Great places to run \\
\hline $26 \mathrm{NP}$ & NP supports active lifestyles through great facilities, people want to live here \\
\hline $27 \mathrm{NP}$ & Coastal walkway main driver for walking/cycling, not MCP \\
\hline $29 \mathrm{NP}$ & Everyone has access to great scenery \\
\hline
\end{tabular}




\begin{tabular}{|c|c|}
\hline $37 \mathrm{NP}$ & Dynamics of city have changed completely \\
\hline $38 \mathrm{NP}$ & Coastal walkway has improved \\
\hline $43 \mathrm{NP}$ & "The people love it!" \\
\hline $45 \mathrm{NP}$ & Great at promoting youth to walk/cycle \\
\hline $46 \mathrm{NP}$ & Gives NP more opportunities to host events \\
\hline $54 \mathrm{NP}$ & Great source of community pride \\
\hline $61 \mathrm{NP}$ & Works well for less confident cyclists \\
\hline $62 \mathrm{NP}$ & Great separated routes, could use more! \\
\hline $63 \mathrm{NP}$ & Motorists attitudes towards walkers and cyclists have improved \\
\hline $73 \mathrm{NP}$ & Overdone, waste of ratepayers' money, biased against motorists \\
\hline $77 \mathrm{NP}$ & Seems to make everyone cheerful, friends from outside NP love it \\
\hline $79 \mathrm{NP}$ & Visitors love Coastal Walkway, few cities have such nice places to walk \\
\hline $83 \mathrm{NP}$ & Central in bringing the community together \\
\hline $84 \mathrm{NP}$ & "Coastal Walkway is the beating heart of New Plymouth" \\
\hline $85 \mathrm{NP}$ & $\begin{array}{l}\text { Fantastic community asset, increases linkages to surrounding communities, provides great } \\
\text { opportunity to actively commute to work }\end{array}$ \\
\hline $86 \mathrm{NP}$ & Increased real estate values for homes near facilities, especially the Coastal Walkway \\
\hline $94 \mathrm{NP}$ & "Positive! Happiness! Well-beingness!" \\
\hline $98 \mathrm{H}$ & Gives Hastings a better profile for outsiders \\
\hline $99 \mathrm{H}$ & Brings more events to Hastings \\
\hline $104 \mathrm{H}$ & Brilliant, cyclists safer off roads, unreal change in cycling culture since 2009 \\
\hline $118 \mathrm{H}$ & "Gets bums out of couches" \\
\hline $120 \mathrm{H}$ & Routes are pram accessible! \\
\hline $126 \mathrm{H}$ & Great for Havelock North, less effective in Flaxmere \\
\hline $136 \mathrm{H}$ & $\begin{array}{l}\text { Attitudes slowly changing in favour of cyclists. Didn't think it was safe enough to cycle before } \\
\text { iWay }\end{array}$ \\
\hline
\end{tabular}




\begin{tabular}{|l|l|}
\hline $137 \mathrm{H}$ & Has been great for schools \\
\hline $138 \mathrm{H}$ & Would be nice to see greater usage from low-income areas \\
\hline $139 \mathrm{H}$ & Nice to be able to exercise and get somewhere \\
\hline $143 \mathrm{H}$ & Only bought bikes after $i$ Way facilities were built \\
\hline $164 \mathrm{H}$ & Never would have attempted to cycle from Clive to Hastings without $i$ Way \\
\hline
\end{tabular}

Table 31: Summarised responses to Question \#16

These comments reflect an overall positive users' reception to the MCP. There are strong sentiments that these improved facilities are promoting greater physical activity and more positive attitudes to active travel as a desired means of transportation, as well as recreation. These comments highlight improved levels of accessibility to walking and cycling facilities for all community members.

\subsubsection{Unsolicited commentary from the interviews}

Throughout the intercept survey interviewing process, the majority of respondents were enthusiastic about giving feedback. In many instances, respondents provided commentary that fell outside the intercept survey's design scope. In some cases, this commentary provided interesting insight, both positive and negative, about the MCP intervention and the wider status of walking and cycling in Hastings and New Plymouth. The most useful responses within this feedback were summarised in Table 32.

\begin{tabular}{|l|l|}
\hline Survey \# & Summarised responses \\
\hline $3 \mathrm{NP}$ & Some cyclists go too fast \\
\hline $5 \mathrm{NP}$ & More signage needed \\
\hline $8 \mathrm{NP}$ & On road cycle lanes are poor in comparison to other cities \\
\hline $11 \mathrm{NP}$ & Too few pedestrian crossings in town \\
\hline $17 \mathrm{NP}$ & Cyclists are dangerous \\
\hline
\end{tabular}




\begin{tabular}{|c|c|}
\hline $39 \mathrm{NP}$ & Cyclists need better provision of infrastructure \\
\hline $43 \mathrm{NP}$ & $\begin{array}{l}\text { National government needs to invest more into walkways/cycleways, fears Auckland is } \\
\text { getting an inequitable share }\end{array}$ \\
\hline $44 \mathrm{NP}$ & Preferred to value walking/cycling facilities as "invaluable" \\
\hline $62 \mathrm{NP}$ & There is often broken glass in cycle lanes, has had frequent punctures \\
\hline $65 \mathrm{NP}$ & Cycling culture is still on the fringe of NZ society \\
\hline $86 \mathrm{NP}$ & Will never wear a helmet while cycling on the Coastal Walkway \\
\hline $96 \mathrm{H}$ & Gates at trail heads are a pain to get around as a cyclist \\
\hline $97 \mathrm{H}$ & Crosses Rd off-road shared pathway has a terrible broken glass problem \\
\hline $99 \mathrm{H}$ & Not a fan of on-road cycle lanes, will only cycle on separated pathways \\
\hline $102 \mathrm{H}$ & $\begin{array}{l}\text { Bicycles will never replace cars in Hastings, however they should be a complementary form } \\
\text { of transport }\end{array}$ \\
\hline $105 \mathrm{H}$ & Wants a safe passage for cyclists on the Chesterhope Bridge \\
\hline $106 \mathrm{H}$ & Parents did not allow her to cycle until $i$ Way infrastructure was built \\
\hline $120 \mathrm{H}$ & Would have walked her kids had the infrastructure been around when they were young \\
\hline $122 \mathrm{H}$ & Has only put $4500 \mathrm{~km}$ on a truck he bought two years ago \\
\hline $126 \mathrm{H}$ & $\begin{array}{l}\text { Suggests lower socioeconomic groups are harder to convince to walk/cycle for exercise as } \\
\text { culturally they often prefer sport }\end{array}$ \\
\hline $127 \mathrm{H}$ & $\begin{array}{l}\text { Suggests lower socioeconomic groups decision to walk/cycle is hindered by negative } \\
\text { perceptions of safety as a result of crime and problematic neighbourhood dogs }\end{array}$ \\
\hline $130 \mathrm{H}$ & $\begin{array}{l}\text { Doesn't understand how as a pedestrian, shopping in the Hastings CBD can be such an } \\
\text { unpleasant experience }\end{array}$ \\
\hline $132 \mathrm{H}$ & $\begin{array}{l}\text { Cycling facilities don't compare to what he was able to use in while living in Europe and } \\
\text { Japan }\end{array}$ \\
\hline $136 \mathrm{H}$ & Didn't think it was safe enough to cycle before $i$ Way \\
\hline $151 \mathrm{H}$ & Knocked off his bike by a car in 2008 . Would like to see more segregated routes. \\
\hline $153 \mathrm{H}$ & As a motorcyclists and a cyclist, has a lot of experience with the perils of inattentive drivers \\
\hline
\end{tabular}

Table 32: Summarised unsolicited commentary 
Comments here provide some interesting criticism of the MCP. Comments suggest that the growing popularity of off-road shared pathways is creating some tension between walkers and cyclists. Several comments were made suggesting that walking experiences in both CBDs have not really improved. Also noted was the idea although cycling facilities have been improved, there is still potential to significantly improve coverage and level of service in both cities.

\subsubsection{Sample of user narratives}

Standard economic evaluation practice aims to assess aggregate benefits from an intervention. This process, which focusses on the collective benefit, can overlook the potential benefit to those users who have unique circumstances who may benefit more from an intervention than an average user. It may also ignore dissenting viewpoints of those who either do not benefit or do not believe this investment is in their community/society's best interest. This section includes 10 short narratives of individuals (and two couples) whose circumstances or beliefs are exceptional in comparison to the collective group and merit some additional reflection. These narratives are based on responses from the intercept survey itself as well as additional unsolicited feedback provided by the respondent. Comments are summarised unless quotation marks are present.

\section{Respondent \#3}

This respondent from New Plymouth is a male cyclist in his 40s. He was not a regular cyclist in 2009. In 2013, he reported cycling on average $70 \mathrm{~km}$ per week. His job requires him to work night shifts, to which he claims had always caused him issues sleeping. He says Let's Go inspired him to cycle to work most days. Since beginning to cycle to work, he claims that his sleeping issues have virtually disappeared, as well as giving him an improved general sense of health and well-being. He says he drives a lot less than before, and is even considering selling his car as he's using it less and less. This respondent was wearing a medic alert bracelet, although he did not mention any medical condition. 


\section{Respondent \#7}

This respondent from New Plymouth is an 85 year old walker. He regularly walked 20 $\mathrm{km}$ per week in 2009, although this has dropped to about $14 \mathrm{~km}$ per week in 2013 . He cites his advancing age as an increasingly limiting factor, and the reason he walks less now than he did four years ago. He enjoys his regular walks along the Coastal Walkway, especially the social nature of being able to communicate with other users. He noted that in recent years, a greater proportion of people he talks to are from out of town or overseas. He notes a few concerns: cyclists ride to fast on shared pathways, and "riff raff" often leave broken glass on the pathways.

\section{Respondent \#30}

This respondent from New Plymouth is a walker in his 30s. He was not a regular walker in 2009, but in 2013 reported 10 hours of walking per week. He did not offer much additional feedback outside of the intercept survey questions, however it is worth noting that he was pushing a profoundly physically and mentally handicapped male in a wheelchair, also in his $30 \mathrm{~s}$.

\section{Respondent \#61}

This respondent from New Plymouth is a cyclist in his 50s. He reported cycling on average $10 \mathrm{~km}$ per week in 2009 and $20 \mathrm{~km}$ per week in 2013. Canadian by birth and also having previously resided in Christchurch, he claims to have been a regular cyclist since he was a teenager. He claims the infrastructure itself has not influenced how much he rides as he is a confident enough cyclist to cycle in any environment. He applauds the Let's Go project for providing better infrastructure and education for less confident cyclists. He recognises the rising tension between walkers and cyclists due to the increasing popularity of the Coastal Walkway. He suggests walkers and cyclists will just have to learn to co-exist, and thinks that lanes lines on the Coastal Walkway would do little to improve the situation. 
These respondents are a couple who are cyclists in their 30s living in New Plymouth. The male originally comes from Germany and the female comes from the Czech Republic. They were not living in New Plymouth in 2009, but reported cycling on average 40 and $35 \mathrm{~km}$ per week. Although they recognise the MCP as significantly improving cycling facilities in New Plymouth, they suggest the level of service this new infrastructure provides is still significantly inferior to what they were used to in their countries of origin.

Respondent \#73 and \#74

These respondents are a couple from New Plymouth who are walkers in their 60s. They both reported walking $5 \mathrm{~km}$ per week in 2009, and the same average distance in 2013. They both offered a largely negative assessment of Let's Go, with the husband claiming that it was "overdone" and "a waste of ratepayer money". He believes that it is "empowering lycra clad maniacs that ride three abreast" and is "biased against motorists". He questioned me to find out how much the programme cost, and how much of that the NPDC had to pay for.

Respondent \#99

This respondent is a cyclist from Hastings in her 60s. Prior to $i$ Way, she would have never considered cycling in Hastings. She now cycles on average two hours a week, often with a neighbour and close friend. Where possible, she rides on separated shared pathways as she believes the roads in Hastings are too dangerous to ride safely on.

\section{Respondent \#107}

This respondent from Hastings is a cyclist who stated he was 80 years old. He claimed to cycle on average $100 \mathrm{~km}$ a week in 2009 and cycled on average $140 \mathrm{~km}$ per week in 2013. He reports that he'll likely lose his driver's licence in two years and hopes to be able to continue cycling whether that happens or not. He reports that he feels the $i$ Way 
infrastructure allows for safer riding, although he expressed concern that the cows near Waitangi Bridge in Pakowhai are no longer fenced in and are now often on the path.

Respondent \#113

This respondent from Hastings is a cyclist in his 30s and is of Maori ethnicity. He reported cycling on average 30 hours a week in 2009, and claims that this has increased to 40 hours in 2013. He was unaware of the $i$ Way programme, but acknowledged that cycling facilities have improved in recent years and that cycling routes now seem safer. He quite proudly reported that he has never had a driver's licence, never owned or a car nor ever plans to own one, and is able to cycle and happily does so to most of his destinations. When asked to put a personal value on the cycling facilities, he replied "you can't put a monetary value on our own health mate”.

\section{Respondent \#139}

This respondent from Hastings is a cyclist in his 60s. He was not a regular cyclist in 2009, citing that before $i$ Way he was not confident enough to cycle in the Hastings area. Prior to 2009, he suffered a stroke, as a result of which he lost his driver's licence and still has some lingering physical and cognitive side effects. In 2013 he reported cycling on average $45 \mathrm{~km}$ a week. He only cycles on $i$ Way's cycling infrastructure, and is appreciative of being able to cycle to many destinations within safe cycling routes, permitting him to exercise and to get around town.

\subsection{Conclusion}

There are numerous co-benefits to active travel, several of which this study has investigated. It is important to note that this study does not provide a complete analysis of all possible benefits. Modal shift to active travel is widely recognised to reduce traffic congestion in urban areas. Given active travel's relatively small modal share compared to motor vehicle use, assessing reduced congestion as a result of increased intake of walking and cycling is difficult. Increased uptake in active travel is also increasingly acknowledged to provide numerous benefits to local communities, including but not limited to 
walkability, liveability, and increased social capital. Given the wide array of potential community benefits associated with active travel and the inherent difficulty in measuring them, this study is only able to briefly evaluate some of these benefits. Community centred benefits should not be underestimated and would greatly benefit from additional attention in future research, notably in the New Zealand context. Despite its limitations, this study has provided a range of insights into how walkers and cyclists benefit from active travel interventions, a matter that to date has received a relatively small amount of attention on the part of transportation policy makers and researchers in New Zealand. 


\section{Chapter 8 - Model Communities Programme project managers' feedback}

\subsection{Introduction}

This study involved interviews with MCP project managers (see Appendix $\mathbf{F}$ for full interview schedule) for each district council. These interviews served two purposes: the first being an opportunity to collect accurate financial information regarding project costs, the second being a chance to gather feedback concerning successes and weaknesses of the MCP from project managers. This chapter focuses on the latter goal with the intention of providing feedback concerning the project managers' experiences through the course of the MCP interventions. Like the intercept survey interview structure, the project manager interview was semi-structured. The interview focussed on the scheduled questions, although it allowed for additional relevant discussion that arose during the interview process. Both project managers were generous with their feedback, and in some instances feedback outside of the interview design's scope was recorded.

It is important to note that analysis of project weaknesses is not intended to be interpreted in any way as scrutiny of the project manager's job performance. Previous national investment in walking and cycling schemes have been small scale and have centred on individuals improving individual routes (NZTA, 2009a). To this author's knowledge, there are no previous comparable cases of medium to large-scale national investment in walking and cycling schemes in New Zealand. The time frame from district council application to NZTA to project completion itself was less than five years total, implying a lot of planning and construction was completed within a very narrow timeframe. It is important to note that initial project costs totalled less than NZ\$ 13 million, a modest investment in comparison to infrastructure costs for other transport projects. NZTA itself has implied that this type of large-scale investment in walking and cycling was designed to be a learning opportunity for transport and urban planners at national and local levels (NZTA, 2013b). An NZTA stipulation for receiving the MCP funding asked that both district councils make themselves available for consultation with other transportation authorities to share their "lessons learned". This chapter aims to provide additional insight into the MCP process from a project manager's perspective. For the sake of simplicity, responses from the New Plymouth MCP project manager will be coded "NP PM" and the Hastings MCP project manager will be coded "H PM". 


\subsection{Project managers responses to questions related to the Model Communities Programme successes and weaknesses}

\section{“(iv) Were any components of the programme unexpectedly challenging?”}

In reference to this first part of question (iv), the H PM was surprised at the difficulty of getting consent to build pedestrian crossings on roads regulated by NZTA. He explained that building pedestrian crossings along these routes requires evidence that there is demand for these facilities. The H PM further expressed concern for the lack of pedestrian crossings near schools in Hastings. He also mentioned that the existing car orientated development of Hastings has created a culture where an undesirable number of motorists are not giving way to pedestrians at the few pedestrian crossing facilities that exist, even for those in close proximity to schools. The H PM also suggests that it was more difficult than expected to motivate non-cyclists to start cycling, although he suggests that after a lot of effort promoting cycling in Hastings, a cycling culture has begun to emerge.

The NP PM noted that a lot of effort was spent on promoting walking and cycling in workplaces across New Plymouth. He further noted that smaller businesses especially those in retail were less enthusiastic about these workplace interventions than he had anticipated. The NP PM also mentioned that the Let's Go project received varying levels of support from senior management as staff fluctuations occurred at NPDC, although he mentioned that in general this support has trended upwards.

\section{“(iv) (a) Were any components of the programme more expensive than expected?”}

In response to part (a) of question (iv), the H PM found that the cost of road line removal was higher than originally anticipated, and to compensate for this, project spending had to be limited in a few other areas.

The NP PM noted that shared pathways often required additional infrastructure such as retaining walls and bridges, which were costlier than expected. The NP PM stressed the desire for infrastructure to be built at a high standard, which naturally at times resulted in higher than anticipated project costs. 


\section{(iv) (b) "Were any components of the programme less costly than expected?"}

In response to part (b) of question (iv), the H PM mentioned that the route to Clive was originally planned to be paved, but as result of cost escalation for other project components, the decision was made to use limestone instead. He adds that this has permitted the use of a locally sourced material at a substantially lower cost, while still proving a high level of service to most users.

The NP PM found that Let's Go events were cheaper to organise than anticipated. He attributed this to a high level of community and corporate support. Local media, especially newspapers, regularly provided free or low cost marketing for Let's Go events, and the local police provided support where needed.

(v) "Which socio-demographic group does the council believe has benefitted the most from this project? (eg age, gender, income, ethic and other groups)”

Both project managers found that schools from communities of lower deprivation were quite responsive to the intervention. The NP PM further added that several larger workplaces in town were very supportive of the workplace interventions, and thus they have seen more active participants than other workplaces.

\section{(v) (a) "Is there a group which has so far benefitted less than most?"}

Both project managers found that schools from communities of higher deprivation were less responsive to the intervention. The NP PM added to this by saying that schools from higher deprivation communities may not have the resources to take on these programmes, often in terms of having staff who have extra time to support the school programmes.

The H PM further stated that higher deprivation communities in general were less enthusiastic about the intervention and also remarked that access to bicycles is less obvious for lower income individuals. The NP PM pointed out that some individuals' car use is such a strong habit that an intervention is unlikely to change this habit. The NP PM also mentioned that the intervention was comparatively less successful for older students in high schools, as the ability to drive a car now offered them a competing form of transport. 


\section{(ix) "In hindsight, if you were starting afresh promoting active travel in this city, how would you approach it differently this time around?”}

Several of the responses to this question were unexpectedly similar from both project managers. Both project managers stressed that their programmes focussed on promoting walking and cycling to the general population. They were satisified that this strategy had maximised exposure of the intervention, and thus resulted in a significant shift in not only walking/cycling numbers, but also improved perceptions of walking/cycling with their districts. Both project managers stated that cultural acceptance of walking/cycling has improved significantly within their districts.

As a consequence of promoting to the general population, both project managers acknowledged that the intervention has been less effective at motivating indivuals from higher deprivation areas to walk or cycle, despite the presence of MCP infrastructure and promotion/education activities in these areas. Although project managers were pleased with the overall success of their promotion and education programmes, they did recognise the limitations of a "one size fits all" approach to promoting walking and cycling, with limited success for communities in higher deprivation areas. Both project managers were at the time of the interviews planning concentrated future efforts at promoting walking/cycling to higher deprivation communities and schools. On another note, both project managers acknowledged that they had worked with their District Health Boards (DHBs) on a few components of the intervention, but a lack of alignment over intervention strategies and roles between Let's Go/i Way project teams and the DHBs prevented any significant collaboration between the two parties.

The H PM also acknowledged feeling pressured to demonstrate that the intervetion was showing success early on in the programme. He acknowledged that measuring progress for walking and cycling in the early stages is difficult, especially for a programme that has limited baseline data and new infrastructure that is spread out across the district council. In hindsight, he would have worried less about trying to demonstrate these successes so early on in the programme.

The NP PM stated that he would have liked more time to garner political support for the programme as the application process itself did not permit a lot of time for this. He also added that he would have liked to have given more attention to promoting active trips for recreational purposes as well as promoting the MCP to active users other than walkers and cyclists. He also mentioned that his team anticipated but underestimated the initial 
speculation that the programme was designed solely to cater to the "supremely fit" and the "wearers of lycra". He added that early on the media fed this speculation, but over time the media has become quite sympathetic to the programme's focus on improving facilities for all potential users. The NP PM also mentioned that certain facility improvements have been at the expense of other users. For example, improvements to the Huatoki Walkway have seen the path smoothed over. Although this was better for most walkers and utilitarian cyclists, trail runners and mountain bikers were not necessarily as enthusiastic about these improvements. The NP PM said he wished these issues had been better anticipated in order to consult affected groups earlier on in the process. The NP PM also stressed the importance of developing good relationships with the State Highway managers and the district council parking enforcement/management teams. He stated that walking and cycling projects can conflict with these agencies' priorities; thus anticipating these can minimise disruptions to certain projects.

\section{(x) "What would you identify as particular successes and weaknesses of the Model}

\section{Communities Programme in your district to date?"}

The H PM reported the programme's biggest success as reversing a trend of decreasing rates for walking and cycling and it has seen a significant rise in active travel users within the Hastings District. He is also quite satisfied with the improvement in community perceptions for walking and cycling as an acceptable and desirable mode of transport. He claims that early on in the programme he fielded numerous calls and letters from local residents expressing concern that $i$ Way would be a waste of ratepayer funds. Three years later he says these negative phone calls have effectively stopped.

The NP PM reported that local iwi have used the improved facilities and increased number of users as an opportunity to build a few signs telling the story of their people's history on the land. He also added that other users such as runners, push-scooters, skateboarders, rollerbladers, wheelchair and mobility cart users can be seen regularly using the infrastructure. Other successes mentioned by the NP PM include positive contributions from accessibility groups to the Let's Go project, the awareness and the strength of the Let's Go brand, several successful Let's Go sponsored community events, as well as increased bus ridership.

In terms of programme weaknesses, the H PM has stated that the team had hoped to improve walking facilities in the city centre, largely through increasing the number of 
pedestrian crossings. As mentionned before, NZTA guidelines has made this a challenging process, a result of which has been fewer pedestrian crossings built than he had previously hoped for.

The NP PM further mentioned that he had hoped the programme would convey a message to employers and retailers that there is an oversupply of parking in the city centre of New Plymouth. He has noted that, in general, there is still resistance to the idea of removing car parks in New Plymouth to make room for other purposes (or other users). The programme has been less effective in this regard than he had originally desired. He is also slightly worried that the Let's Go brand has a perception of being tied to "gym bunnies" and "the super fit", and thus is potentially off-putting to the less fit individuals who would have a lot to benefit from the programme.

(xi) "What would you see as the pros and cons of cost-benefit analysis as a means of assessing the effectiveness of the Model Communities Programme in your district?”

(xi) (a) "In your opinion, what are the attractions of cost-benefit analysis in evaluating the overall meris of your programme?”

The NP PM responded that CBA allows for an easy comparison of the potential values of one project in comparison to another, ideally allowing for a more thorough assessment of potential projects. Due to the nature of national funding for transportation projects in New Zealand, it is an essential process for receiving funding for most transport projects.

(xi) (b) "In your opinion, what are the limitations of cost-benefit analysis in evaluating the overall merits of your programme?”

The H PM stated that CBA in the transport domain in New Zealand today does not adequately address factors associated with quality of life, social capital and other community benefits that are central to programmes that promote walking and cycling. It is nearly impossible to capture the value of providing an environment that is pedestrian friendly, where a child is safe to grow up in and explore, or even to place a value on a cohesive community. He also argued that there is a significant social cost to motor transport in New Zealand, one that is largely undervalued by transportation authorities today. 
The NP PM stated that CBA as practised today does not accurately reflect total benefits achieved from interventions that promote walking and cycling. These undervalued benefits include: encouraging schoolchildren to develop healthy habits from an early age, children's decreased reliance on parents for their transport needs, greater accessibility (notably for vulnerable population groups), creation of an environment that encourages social interaction between community members and the provision of scenic walking/cycling routes that can be enjoyed by all. He also noted some economic benefits that are difficult to account for in conventional CBA including: greater potential for tourism, attracting more community events and creating a more desirable place to live to attract a greater number of skilled workers and potential employers to the New Plymouth District. He also cited some new housing developments being purposely built around, and integrating themselves with, existing walking and cycling facilities, as these areas are becoming increasingly desirable to live nearby. 


\section{Chapter 9 - Discussion}

This research set out to evaluate the effectiveness of medium scale investments in walking and cycling interventions in the urban New Zealand context. This research provides three separate evaluations of NZTA's MCP: an economic evaluation in the form of a CBA, an evaluation of users' perceptions of benefits from the intervention using qualitative and quantitative data gathered from an intercept survey; and an evaluation of MCP project managers' perceptions of intervention success and weaknesses using qualitative analysis gathered from interviews with these managers. This chapter discusses the results of these evaluations. It also discusses strengths and limitations of this research as well as recommendations for further research. The chapter concludes with a discussion of policy recommendations that might be drawn from this study.

\subsection{Discussion of results from the CBA evaluations of the MCP}

All CBA evaluations reported BCRs of greater than 1, suggesting that the MCP was estimated to be cost-beneficial even under conservative economic evaluation frameworks. Results varied significantly between CBA evaluations, with benefit-cost ratios ranging from 1.3 to 21.7 (Table 33). All evaluations analysed using quasiexperimental conditions (i.e. with controls) calculated BCR values that were twice the value of the same evaluations calculating the BCRs of the intervention itself (with no control). The difference in values between the BCRs calculated from the quasiexperimental conditions versus those calculated from the intervention cities alone represents the potential benefits in terms of users who might have stopped walking and cycling in the absence of the intervention, given previous declining rates of active travel in the intervention cities. A study by Genter \& NZ Transport Agency (2009) highlighted the diminishing levels of walking and cycling in New Zealand since the early 1980s, and further suggested that economic analysis of walking and cycling interventions should account for the potential number of users who continue to walk and cycle as a result of the improved facilities.

In terms of the distribution of benefits for the two evaluations based on the NZTA EEM framework, Evaluation \#1 recorded 70\% (medium estimate, no control) and 
$81 \%$ (medium estimate, with control) of its benefits as "Composite Benefits", with the remainder of benefits as "Improved Route Safety".

\begin{tabular}{|c|c|c|c|c|c|c|}
\hline & \multicolumn{3}{|c|}{$\begin{array}{l}\text { Intervention BCRs (no } \\
\text { control) }\end{array}$} & \multicolumn{3}{|c|}{$\begin{array}{l}\text { Intervention BCRs (with } \\
\text { control) }\end{array}$} \\
\hline & Medium & High & Low & Medium & High & Low \\
\hline $\begin{array}{l}\# 1 \text { NZTA EEM } 2010 \\
\text { (30 years, } 8 \% \text { discount } \\
\text { rate) }\end{array}$ & 1.3 & 1.5 & 1.3 & 2.9 & 3.1 & 2.6 \\
\hline $\begin{array}{l}\text { \#2 NZTA EEM } 2013 \\
\text { (40 years, } 6 \% \text { discount } \\
\text { rate) }\end{array}$ & 1.7 & 2.0 & 1.6 & 3.7 & 4.3 & 3.4 \\
\hline $\begin{array}{l}\text { \#3 More robust } \\
\text { estimate ( } 40 \text { years, } 3 \% \\
\text { discount rate) }\end{array}$ & 5.3 & 6.3 & 4.8 & 10.9 & 12.6 & 10.4 \\
\hline $\begin{array}{l}\text { \#4 Optimistic Estimate } \\
\text { (50 years, } 1.4 \% \\
\text { discount rate) }\end{array}$ & 8.2 & 10.3 & 7.3 & 17.3 & 21.7 & 15.5 \\
\hline
\end{tabular}

Table 33: CBA simulations summary

When comparing the BCR calculations from Evaluations \#3 and \#4 to those of the evaluations based on the NZTA framework, several aspects stand out. First, the BCR for Evaluation \#3 (no control) is three times that of Evaluation \#2, which reflects NZTA's current economic evaluation framework. Evaluation \#4's (no control) BCR values are over five times as high as Evaluation \#2's BCRs.

There are several reasons why Evaluation \#3 and \#4's BCRs are higher than those from Evaluation \#2, which represents one of the methodologies currently offered under NZTA's 2013 EEM. Although the 2013 EEM has loosened its evaluative conditions in the form of a longer timeframe (40 years instead of 30 years) and a lower discount rate ( $6 \%$ instead of $8 \%$ ), its discount rate is twice the rate of CBA Evaluation \#3. This $6 \%$ rate is higher than those of the four CBAs analysed in Chapter 3. A study by Pettit (2013) noted that not only do the UK and France employ lower discount rates for transportation projects $(3.5 \%$ and $4 \%)$, but these rates begin to diminish after thirty years. If higher discount rates are employed to account for uncertainties in terms of an intervention's actual potential benefits, then NZTA's use of a higher discount rate may be justified for evaluating economic

\footnotetext{
${ }^{1}$ Per km benefits for walkers and cyclists listed in NZTA's EEM. They include health, road traffic
} reduction and safety. 
efficiencies of walking and cycling projects and for other projects related to motor vehicle transport. However, it is also possible to argue that discounting roading projects and walking/cycling projects at the same rate is undesirable. Quiggin (1997) argues that adoption of high discount rates to account for uncertainty is undesirable as in some cases it promotes unsustainable consumption of resources. In effect, discounting motor vehicle and active travel infrastructure at the same rate may provide little incentive for society to choose less resource intense modes of transportation. As discussed in Chapter 3, the benefits of increased rates of walking and cycling to society are becoming increasingly accepted by academics and policy makers while the external costs associated with motor vehicle use are receiving greater amounts of recognition (Craig, 2013; Dora et al., 2000; Lawrence D. Frank, 2004; Litman, 2006; Ruta, 2002). Thus, if one mode of transport is largely beneficial to society while the other imposes a cost to society that is not fully covered by the user, it would seem counter-intuitive to evaluate projects that improve facilities for both modes using the same evaluative conditions.

NZTA's decision to use a lower discount rate for its 2013 EEM (and increase the evaluation timeframe) is clearly a step in the right direction; however its relatively high rate and universal application to all transport projects is still a cause for concern. NZTA's EEM framework seems focussed on maximising economic return, while clumsily allowing for risk and uncertainties, but being subtly biased against alternative forms of transport that (a) provide better social equity and intergenerational outcomes (Boyce, 2010; Damart \& Roy, 2009; Whitzman et al., 2010); (b) promote climate change mitigation (Forsyth \& Krizek, 2010; Giles-Corti et al., 2010; Harwatt et al., 2010); and (c) present substantially lower external costs to society (Dora et al., 2000; Litman, 2006; Ruta, 2002). A 3\% discount rate in Evaluation \#3 and a 1.4\% rate in Evaluation \#4 better take into account the above concerns.

Another key reason Evaluations \#3 and \#4 have calculated substantially higher BCRs is the fact that they allow accrual of two benefits (reduced insecurity and improved journey quality) to the baseline km walked/cycled. NZTA's 2013 EEM allows for composite benefits that can be applied to new $\mathrm{km}$ that are walked and cycled as a result of an intervention. Although this methodology is not uncommon in economic evaluation of walking and cycling interventions, it has a key weakness. Although it is often assumed that baseline $\mathrm{km}$ walked/cycled after an intervention 
provides no additional benefit to society, it overlooks that existing walkers and cyclists can benefit from routes that are safer, smoother, more direct, more convenient and possibly even more scenic. Cyclists in particular stand to benefit given their mode's closer proximity to motor vehicle traffic and comparatively limited provision of mode-specific facilities in the urban New Zealand context. Although the EEM framework accounts for these users' additional km walked/cycled, this author believes it is unreasonable to omit a benefit accruing in terms of baseline $\mathrm{km}$ travelled. In a previous CBA for improved walking/cycling facilities in three Norwegian cities, Saelensminde (2004) estimated a reduced insecurity benefit to new and existing $\mathrm{km}$ walked and cycled to account for improved walking/cycling conditions for both new and existing users. Analogously, the value of improved safety and journey quality benefits for existing users has been estimated here.

When evaluating the distribution of benefits for Evaluations \#3 and \#4, a few matters become apparent. Evaluation \#3's medium estimate (no control) of journey quality benefit accounts for $22 \%$ of the CBA's total benefit and reduced insecurity accounts for $26 \%$ of the total benefit. It may seem unlikely that over $48 \%$ of the total benefits can be attributed to these two benefits. However, current conditions in New Zealand cities need to be considered. Cyclists in most urban New Zealand contexts are not provided with a significant level of service, especially in comparison to urban areas in Denmark, the Netherlands and Germany, or in parts of the UK. Walkers in New Zealand are generally provided with separated footpaths and pedestrian crossways, although routes that are genuinely distanced from motor vehicle traffic, emissions, and noise are by no means readily available in all urban areas. The results of the intercept survey confirm that safety is a key issue for both walkers and cyclists, thus justifying a generous allocation of this benefit. There is substantial literature stressing the importance of perceptions of safety in relation to an individual's decision to cycle (Daley et al., 2007; Forsyth \& Krizek, 2010; Garrard et al., 2008; Hunt \& Abraham, 2006; Ogilvie et al., 2010; Pucher \& Buehler, 2008; Winters et al., 2010), including a study in the context of urban New Zealand by Kingham et al. (2011). A standard safety benefit of NZ\$ 0.05 per new km cycled offered by the 2013 EEM of NZ is unlikely to adequately account for the importance of the issue of safety for urban cyclists. 
There is another peculiarity within the EEM that is indirectly related to cyclists' perceptions of safety. As mentioned in Chapter 3, the NZTA's 2013 EEM provides a Relative benefit for different types of cycle facilities for forecasting cycling demand based on the type of improvement. These "relative benefit" calculations are based on an adapted stated preference (ASP) survey that reported that cyclists surveyed would spend twice as much time on an "off-street cycle path" relative to a baseline facility, or "on-street facility with parking (no marked cycle lane)". However, in comparison to facilities with "marked cycle lanes” present, the estimated marginal benefits of "off-street cycle paths" are small. The relative benefit calculations assume that cyclists are willing to spend just $5 \%$ more time on an "off-street cycle path" than on a marked cycle lane that is "on-street without parking”. Similarly, the values suggest that cyclists are willing to spend only $10 \%$ more time using off-street cycle paths than on street facilities with parking.

The implications of this modest marginal benefit for "off-street cycle paths" should not be underestimated. A recent study by the Clean Air Partnership in Canada estimates that separated bicycling facilities can cost between 4 and 12 times as much as on-road cycle lanes on existing roads (Craig, 2013). NZTA's small relative benefit for "off-street cycle paths" thus compares poorly with an option whose provision comes at a high cost. These framework guidelines will almost guarantee that on-street facilities will be favoured in economic evaluation at the expense of off-street facilities. Cities such as Amsterdam, Copenhagen and Portland are renowned for their high cycling modal shares, and all three cities have also made significant investments in separated bicycle facilities, undoubtedly on the basis of their benefits. Thus it seems counter-intuitive that NZTA would provide such a modest marginal benefit for "off-street cycle paths" in comparison to "on-road facilities”.

The study on which NZTA bases its "relative benefit" calculations ${ }^{1}$ presents several methodologies for forecasting cycling demand. The study also brings up several issues related to forecasting cycling demand. One issue recognised is the significant disparities in cycling modal shares between localities, citing an example of one study showing variance for cycling modal shares (measured from census data) between neighbourhoods in the Minneapolis-Saint Paul metropolitan area which vary between $0 \%$ and 16\% (Krizek et al., 2006). Other issues mentioned include availability of reliable data sets to accurately model cycling demand, the inherent difficulties involved in predicting how many cyclists

\footnotetext{
'Guidelines for Analysis of Investments in Bicycle Facilities, National Cooperative Highway Research Program Report 552, Transportation Research Board
} 
observed are actually cycling regularly and the uncertainties of the relationship between utilitarian and recreational cycling (2006).

NZTA's use of this study to calculate the EEM's "relative benefit for different types of cycle facilities" is of some concern. Krizek et al.'s (2006) report related to an adapted stated preference (ASP) survey of employees of the University of Minnesota. 2500 university employees were invited (via randomised selection) to participate in a study investigating "transport habits to work". Only 168 employees completed the survey. Additional data gathered from this survey showed that within this population sample, $22.6 \%$ of respondents listed cycling as their "usual mode" to work during the summer (2006). Even more impressive, $9 \%$ of respondents reported regularly cycling to work during the winter in a city (Minneapolis) that records mean winter temperatures of $-7^{\circ} \mathrm{C}$, also listed as the coldest of the top fifty cities in the US in terms of population size'. This sample population has a cycling to work modal share for winter that is over 9 times the overall cycling to work modal share of the entire US (2006).

The sample population's unusually high cycling to work modal share is likely explained by several factors. The authors themselves acknowledge previous studies that show a positive correlation between education and individual's willingness to cycle (2006). Given that respondents are all employees of a university, they are likely to have obtained higher academic qualifications, and accordingly would be expected to cycle more than the general population. The authors also recognise that neighbourhoods near universities often show higher rates of cycling than other neighbourhoods, though they add that this is not universally true.

Krizek et al. (2006) present the ASP survey and the other components of a study as robust measures and low-cost solutions to forecasting local cycling demand. The authors state:

The report contains findings for residents of Minneapolis and St. Paul. The team is not convinced that these finding are applicable to other locations. (2006, p.26)

Although not explicitly stated, it seems the original intention of this report is to provide methodologies for forecasting cycling demand that are robust and easily applied to studies in other locations. Referencing within the EEM gives no indication that a study was

\footnotetext{
${ }^{1}$ http://www.currentresults.com/Weather-Extremes/US/coldest-cities-winter.php
} 
carried out using Krizek et al.'s ASP methodology in a New Zealand cycling context, although the EEM seems to imply that an evaluation can include values calculated as part of an evaluation's own stated preference survey. The "relative benefit" values listed in the 2013 EEM appear to be loosely based on Krizek et al.'s ASP results from the survey of employees at the University of Minnesota. Table 34 presents relative benefit calculations made for the present study using the three models presented by Krizek et al. (original mean analysis, logit regression analysis and linear regression analysis) and compares them to the 2013 EEM's listed relative benefits. Referencing in the EEM appears to indicate that its relative benefit calculations are derived directly from Krizek et al.’s report. NZTA's calculations for the baseline facility and "off-street cycle paths" appear to be based on the report's linear regression analysis. However, the source of NZTA's values for the other facilities are not clear. A value of 1.82 for "on-street, with parking, marked cycle lane” from the linear regression model suggests this may be the source, although this would imply that NZTA is "picking and choosing" values between different models. The EEM's absence of a methodology provides no insight into this issue.

\begin{tabular}{|l|l|l|l|l|}
\hline Facility Type & $\begin{array}{l}\text { NZTA EEM } \\
\text { Relative } \\
\text { Benefit }\end{array}$ & $\begin{array}{l}\text { Logit } \\
\text { regression }\end{array}$ & $\begin{array}{l}\text { Linear } \\
\text { regression }\end{array}$ & $\begin{array}{l}\text { Original } \\
\text { Mean }\end{array}$ \\
\hline $\begin{array}{l}\text { On-street, with } \\
\text { parking, no } \\
\text { marked cycle } \\
\text { lane (baseline) }\end{array}$ & 1 & 1 & 1 & 1 \\
\hline $\begin{array}{l}\text { On-street, with } \\
\text { parking, } \\
\text { marked cycle } \\
\text { lane }\end{array}$ & 1.8 & 1.82 & 1.66 & 1.98 \\
\hline $\begin{array}{l}\text { On-street, no } \\
\text { parking, } \\
\text { marked cycle } \\
\text { lane }\end{array}$ & 1.9 & 2.27 & 1.84 & 2.04 \\
\hline $\begin{array}{l}\text { Off-street } \\
\text { cycle path }\end{array}$ & 2 & 2.53 & 1.96 & 2.16 \\
\hline
\end{tabular}

Table 34: Comparing NZTA's 2013 EEM relative benefit calculations for different types of cycle facilities to this author's relative benefits calculated from Krizek et al.'s 2006 report 
NZTA's decision to use Krizek et al.'s sample ASP results is questionable. The cycling preferences for employees of an American university (who cycle to work at a rate of at least three times the share reported by New Zealand census data) are unlikely to reflect the cycling preferences for the average New Zealander. Although the EEM stipulates that evaluators may use their own "relative benefit" values based on their own stated preference survey results, the EEM's provision of base values that are unlikely to reflect cycling preferences in New Zealand is misleading and is likely to underestimate the benefits of off-street cycle paths. This may have the (unintended) effect of significantly reducing the potential for off-street cycle paths to encourage more New Zealanders to become regular cyclists.

The "relative benefit" values used by NZTA themselves represent potentially misleading generalisations concerning the ability of separated bicycle facilities to attract cyclists. Separated bicycle/shared facilities can take numerous forms ranging from: physically separated lanes adjacent to major arterials with fast-moving traffic and intersecting driveways (e.g. the Havelock North to Hastings iWay arterial) to scenic pathways that are largely separated from motor vehicle traffic, noise and emissions (e.g. the Coastal Walkway in New Plymouth). Providing a single relative benefit value for these facilities is unlikely to accurately predict future cycling traffic on these separated pathways.

These aforementioned issues draw into question the robustness of the EEM's existing methodologies for evaluating economic efficiencies for walking and cycling interventions. It would be logical for NZTA to make it a priority to acquire a relevant survey's results within the New Zealand context as the basis for its relative benefit estimates. At the time of this study, the 2013 EEM does not provide a reference list for its methodologies for evaluating walking and cycling interventions. At the very least, the EEM would do well to provide transparent methodologies with appropriate references ${ }^{1}$.

Evaluations \#3 and \#4 provide other substantial benefits that are not explicitly included in the EEM walking and cycling framework. In Evaluation \#3's medium estimate (no control), other benefits include Vehicle Operating Cost Savings (7\% of total benefits), Mortality Savings (37\% of total benefits), Congestion Savings ( $2 \%$ of total benefits),

\footnotetext{
'The 2010 EEM vol.2 incorrectly references the source of NZTA's "relative benefits" values. It took this author five personal communications (emails) to NZTA over the course of six weeks to obtain the correct source of the EEM's "relative benefit" values.
} 
Greenhouse Gas Savings (<1\%), Health Care Cost Savings (5\%), and Reduced Absenteeism Savings (1\% of total benefits). A value of $<1 \%$ for Greenhouse Gas Savings is likely underestimating the actual potential for climate change savings. A recent paper by Dietz and Stern (2014) argues that contemporary use of social costs of carbon regularly undervalues the true costs of greenhouse gas emissions. The social costs of carbon values used in this economic analysis may indeed be undervaluing the MCP's potential Greenhouse Gas Savings.

"Health Benefits" account for about $90 \%$ of the EEM's per km cycling composite benefit and over $95 \%$ of the per $\mathrm{km}$ walking composite benefit. The EEM itself does not clarify its methodology for calculating this health benefit. Within the EEM's composite benefit, a provision of NZ\$ 0.10 is included as a "Road traffic reduction" benefit, but as with the health and safety provisions, no methodology is provided to inform evaluators what this includes or how the value was calculated. Litman (2013a) notes several benefits associated with reduction in automobile travel including reduced traffic congestion, road and parking facility cost savings and reduced air pollution. In the sense of increased uptake of walking and cycling reducing overall motor vehicle traffic, it is also plausible that "road traffic reduction" benefits should also include other community related benefits such as increased community cohesion and social capital as well as improved equity and accessibility travel outcomes. It is unlikely that the EEM's value of NZ $\$ 0.10$ per new $\mathrm{km}$ walked/cycled can adequately reflect this list of benefits.

CBA Evaluation \#4 provided an "Optimistic Estimate", using a wide array of benefits evaluated under a longer timeframe (50 years) at a discount rate $(1.4 \%)$ that should better account for social and intergenerational equity including risks associated with climate change, as per Stern (2006) and Ackerman \& Stanton (2010). The highest BCR estimate taking into account declining rates of walking and cycling in the control cities was 21.7. This estimate is high and would likely be received with scepticism by policy advisers. It is important to note that this BCR reflects benefits gained from a relatively modest investment that according to Census data has reversed declining active travel rates and achieved significant gains in cycling in the intervention cities. The number of cycling trips to work in the intervention cities increased by $20.7 \%$ from the 2006 to the 2013 census days compared to declining by $17.7 \%$ in the control cities. One can argue that the potential for savings from health, safety, road traffic reduction and community related benefits in a region that is experiencing declining rates of active travel could be substantial. 
Evaluation \#3 represents a "More Robust Estimate". Using the CBA that evaluates the intervention without the benefit of a control achieves a medium estimated BCR of 5.3, a value which would be considered a high priority value by NZTA. Unlike the EEM, the approach taken uses a more transparent framework presenting several benefits that can be attributed to increased uptake of walking and cycling. However, it is important to note that it is not intended to be a complete evaluation of the MCP. It does not explicitly include community related benefits such as liveability, decreased community severance, increased community cohesion, and increased accessibility for vulnerable groups including children, the elderly and the disabled. These benefits are addressed in the next section.

\subsection{Discussion of results from the mixed method intercept survey analysis and project manager qualitative interviews}

MCP user feedback from the intercept surveys (Chapter 7) and project manager feedback (Chapter 8) provided insight into users' and project managers' perceptions of benefits as a result of the MCP. This analysis provides further evidence of the potential benefits from large scale investment in active travel interventions.

\subsubsection{Respondents' gender and ethnicities, pre and post intervention}

MCP users surveyed $(n=100)$ were disproportionately male $(62 \%) .51 \%$ of walkers surveyed were male compared to $71 \%$ of cyclists surveyed who were male. This higher proportion of cyclists surveyed who were male is consistent with trends noted by Garrard et al. (2008) and Kingham et al., (2011) which were that females are disproportionately less likely to cycle in countries with low overall rates of cycling. Results of a survey by Garrard et al. (2008) performed in Melbourne found that within a survey sample of almost 7,000 cyclists, over 79\% were male. Additional analysis of the demographic data from the MCP intercept survey shows that $78 \%$ of cyclists who were regularly cycling in Hastings or New Plymouth in 2009 were male (Figure 46). The 2013 proportion of cyclists who were male, at $71 \%$, therefore represents a decline (Figure 47). It is possible that the intervention has created a slight improvement in the perception of cycling amongst females, although a relatively small sample size for the 2009 estimate $(n=36)$ is unlikely to make this a statistically significant change. 


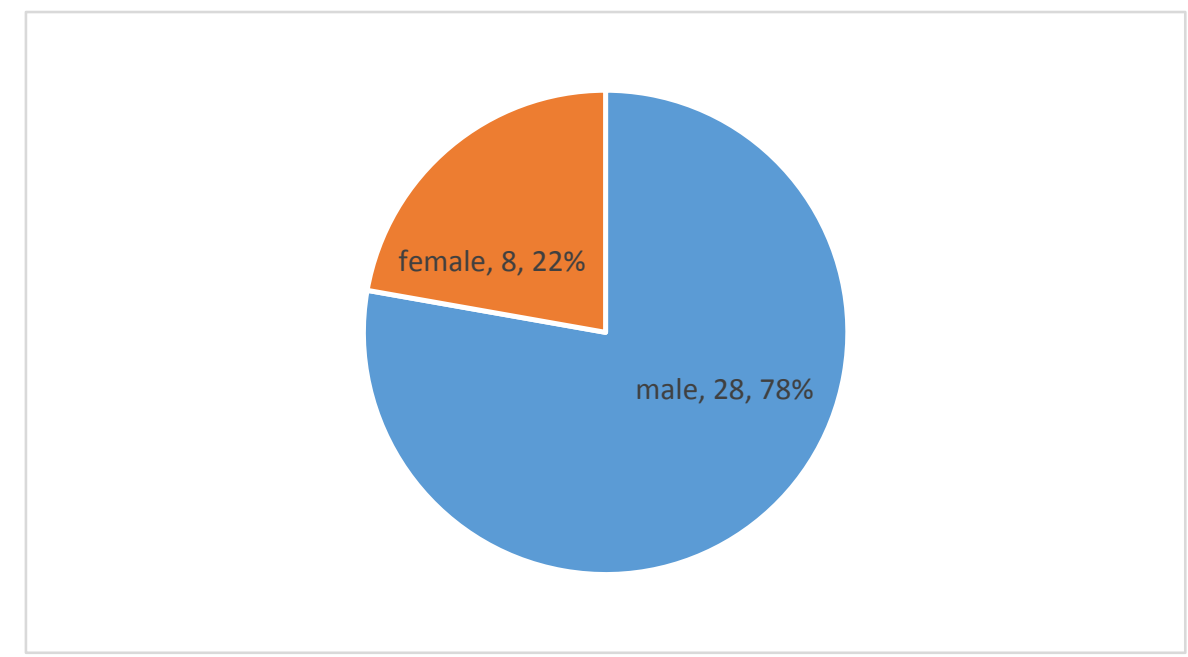

Figure 47: Gender of cyclists who reported cycling in 2009, $n=36$

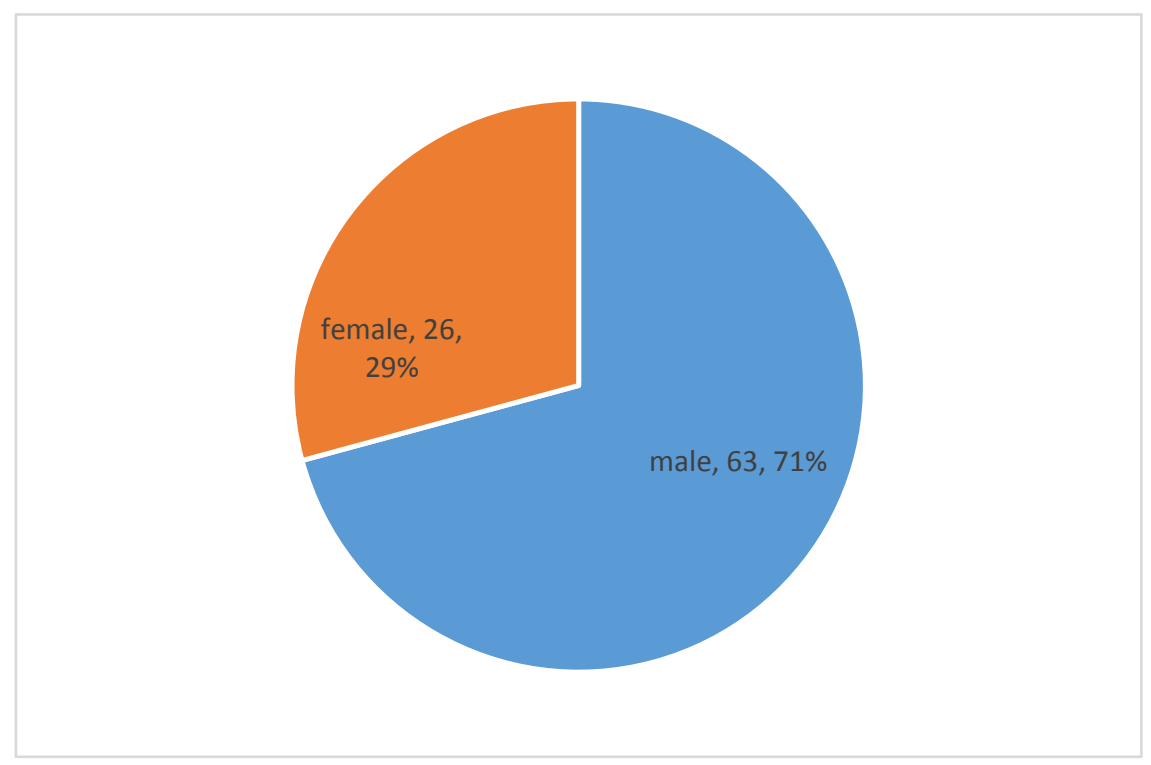

Figure 46: Gender of cyclists who reported cycling in 2013, $n=89$

In terms of ethnicity, the overrepresentation of Europeans compared to the underrepresentation of minorities (Maori, Pasifika, Asian) is not surprising. A report by Krizek et al. (2006) found strong positive correlations between bicycling rates and income/education/low community deprivation, factors that are often associated inversely with minorities. Project manager feedback suggested that accessibility to bicycles was less obvious for groups of lower socioeconomic status. The project managers also mentioned that in general, lower deprivation communities were more receptive to the intervention as 
schools and workplaces in these areas had more resources to take on the MCP educational/promotional components. Respondent \#126 from Hastings suggested that the intervention has been less successful in lower deprivation communities as residents within these areas have a preference towards achieving physical activity through sport, rather than walking or cycling. Respondent \#127 from Hastings suggested that lower participation rates from higher deprivation communities may be an issue of safety, as crime and problematic neighbourhood dogs discourage regular walking and cycling in these areas. This latter argument is similar in nature to that made in a 2011 study by Joh et al. (2011). The project managers have acknowledged that 'one-size fits all' approaches to promoting walking and cycling have had limited success in higher deprivation communities, which Ogilvie et al. (2010) describe as groups that require targeted interventions to meet their unique needs.

\subsubsection{Discussion of respondents' levels of walking, pre and post intervention}

Data from the intercept surveys show that $66 \%$ of walkers reported walking regularly in Hastings or New Plymouth in 2009, 19\% reporting that they lived outside of Hastings/New Plymouth in 2009, and 12\% stating they were not regular walkers in 2009. In comparison, only $40 \%$ of cyclists reported regularly cycling in 2009 , while $29 \%$ were not regularly cycling in 2009 and 31\% were not living in Hastings/New Plymouth in 2009. These data suggest that a larger proportion of cyclists were not regularly cycling prior to the intervention in comparison to walkers. This could imply that cycling was comparatively less common than walking prior to the intervention.

Walkers reported walking $38.3 \%$ more kilometres on average in 2013 than in 2009. Cyclists on the other hand collectively cycled $15.4 \%$ less in 2013 compared to 2009. However, when limiting the analysis to cyclists who were regularly cycling in 2009, these same cyclists cycled $15.5 \%$ more in 2013. Effectively, those who started cycling after the start of the intervention in Hastings and New Plymouth were not 'devoted cyclists' - they reported cycling $44.6 \%$ less than those who cycled before the intervention. This lower figure likely reflects the increase in numbers of casual cyclists and cyclists who cycle to work, both users who would likely have lower rates of cycling than experienced recreational cyclists. This suggests that walking and cycling levels are likely increasing due to new users, and not solely from existing users walking and cycling more, an issue raised 
by Ogilvie et al. (2004) as a common weakness in evaluating walking and cycling interventions.

A paired t-test was performed to evaluate whether or not the reported increases in walking and cycling (from respondents who were regular users in 2009 and 2013) were statistically significant. The reported increase in walking distances was statistically significant $(\mathrm{t}(49)=$ -2.442, $\mathrm{p}=0.018)$ whereas the reported increase in cycling distances was not ( $\mathrm{t}(35)=$ 1.311, $\mathrm{p}=0.198$ ). As all walking and cycling distances were user reported estimates, these results should be interpreted with some level of scepticism.

\subsubsection{Users' ratings and rankings of facilities}

Users who reported being regular walkers in 2009 and 2013 reported modest increases in ratings. The "Coverage" criterion changed the most, by a full point, while "Personal Safety" only increased by three tenths of a point (Figure 47).

If walkers' changes in ratings of facilities from 2009 to 2013 might be described as modest, cyclists' changes in ratings of the six criteria are far more emphatic. "Separation from Motor Vehicles" had by far the largest increase in ratings (3.4 points) while "Motorist Attitudes" achieved the smallest increase ( 0.9 points), as seen in Figure 48. Cyclists by and large seem to have rated their facilities comparatively poorly prior to the intervention compared to walkers' ratings. Although cyclists' ratings increased dramatically in comparison to walkers' ratings, walkers' 2013 ratings exceeded cyclists' 2013 ratings for every criterion. This suggests that, although cycling facilities have improved considerably as a result of the intervention, their corresponding level of service may be perceived as lower than the level of service provided by walking facilities. This proposition is supported by commentary from respondents \#62 and \#63, who claim that the level of service provided by the new MCP facilities is an improvement, but still considerably inferior to what they had while living in Europe. Further investigation of this would be a matter of research interest. 


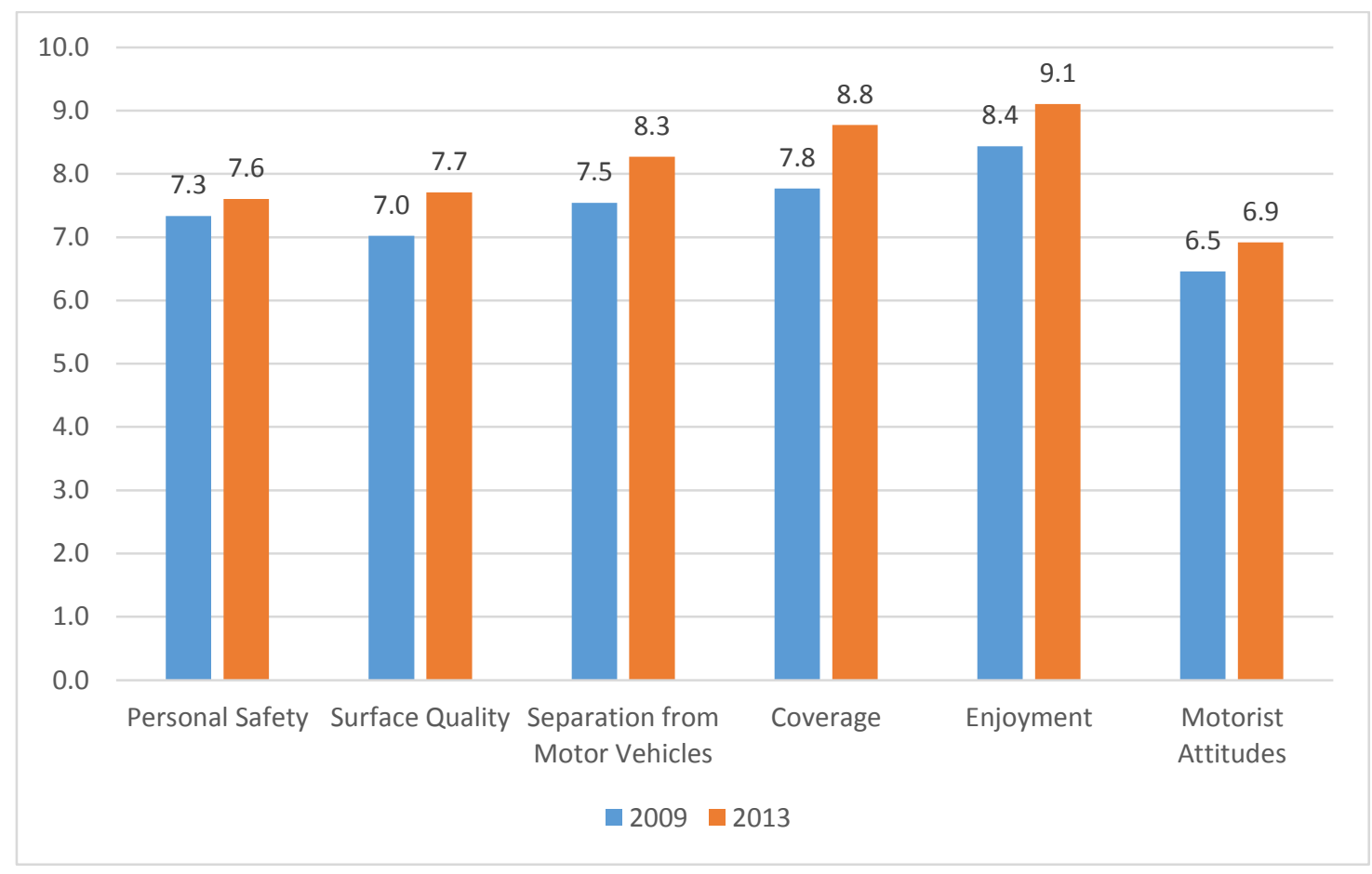

Figure 48: Walkers' perceptions of walking facilities before and after MCP, 2013 walkers who were also walkers in 2009, $n=48$

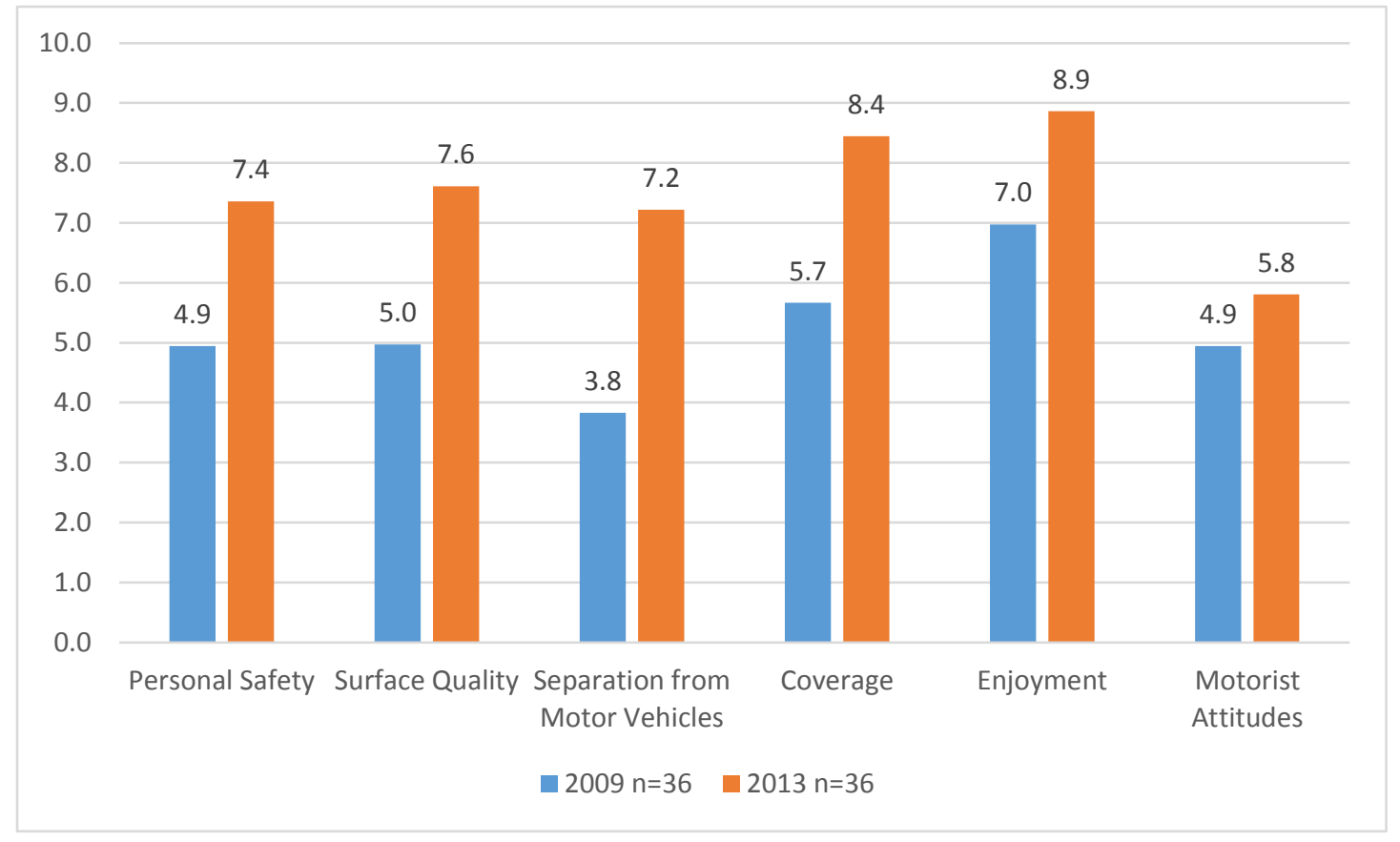

Figure 49: Mean 2013 ratings of cycling facilities in Hastings and New Plymouth from cyclists who were regularly cycling in 2009, $n=36$ 
In terms of rankings, there are notable differences in which criterion walkers and cyclists ranked as most important to them. About $38 \%$ of walkers ranked "Enjoyment" as their most important criterion, compared to $7 \%$ of cyclists (Figure 50 ). $41 \%$ of cyclists ranked "Separation from Motor Vehicles" as the most important criterion to them compared to only $3 \%$ of walkers. This result strongly underlines the relative value of separation for cyclists that we have emphasised above. $39 \%$ of walkers and $33 \%$ of cyclists listed "Personal Safety" as their most important criterion suggesting that this is a key criterion for both users. Both users also provided low frequencies of \#1 rankings for "Motorist Attitudes”.

Both walkers and cyclists seemed relatively insensitive to "Motorist Attitudes" as this criterion received the highest frequency of \#6 importance rankings for both users (Figure 51). "Separation from Motor Vehicles" received the lowest frequency of \#6 rankings for cyclists (underscoring that it is never seen as unimportant) while "Personal Safety" received zero \#6 rankings for walkers, confirming that it is a key criterion for them.

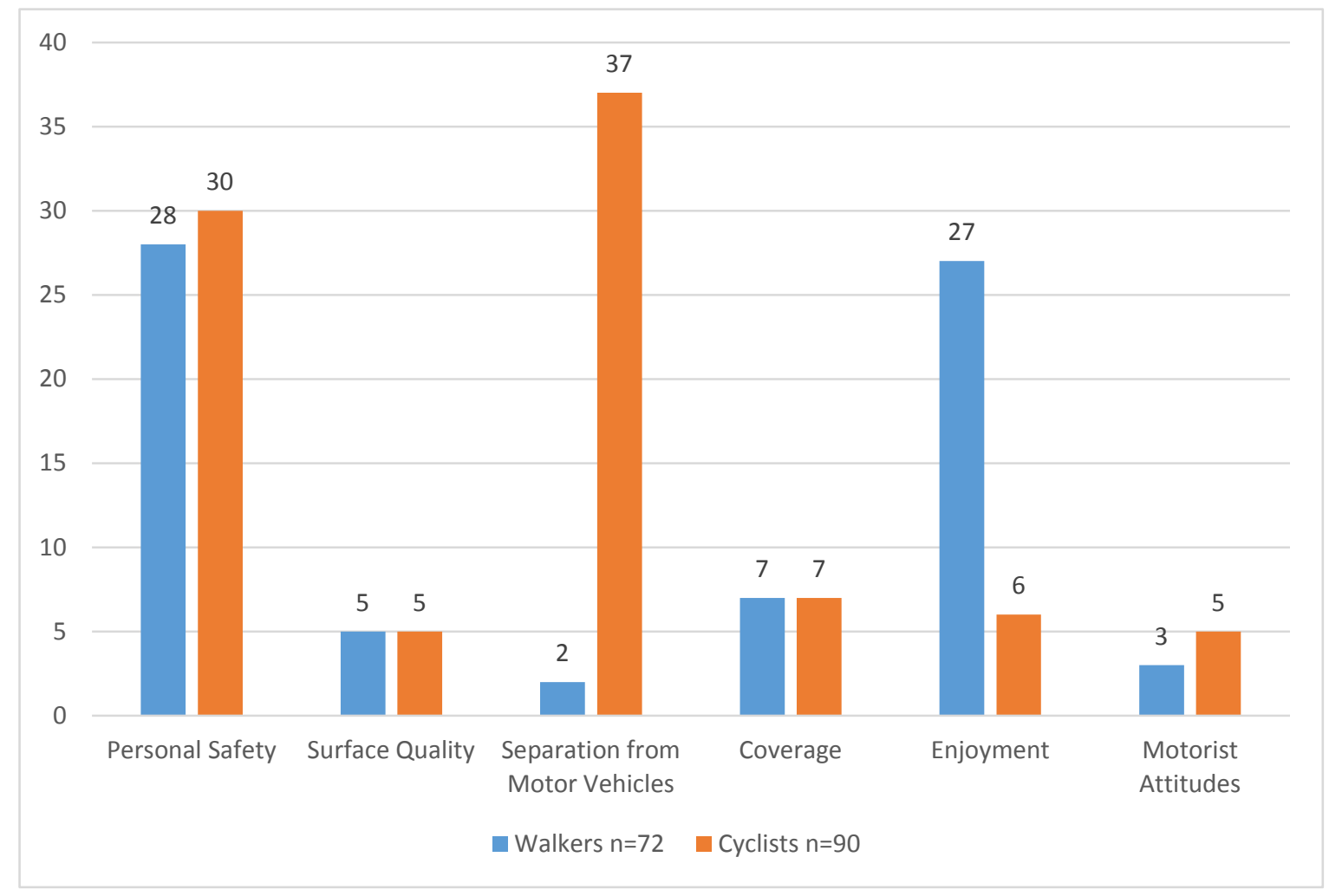

Figure 50: Frequency of \#1 rankings for walkers and cyclists per criterion in 2013 


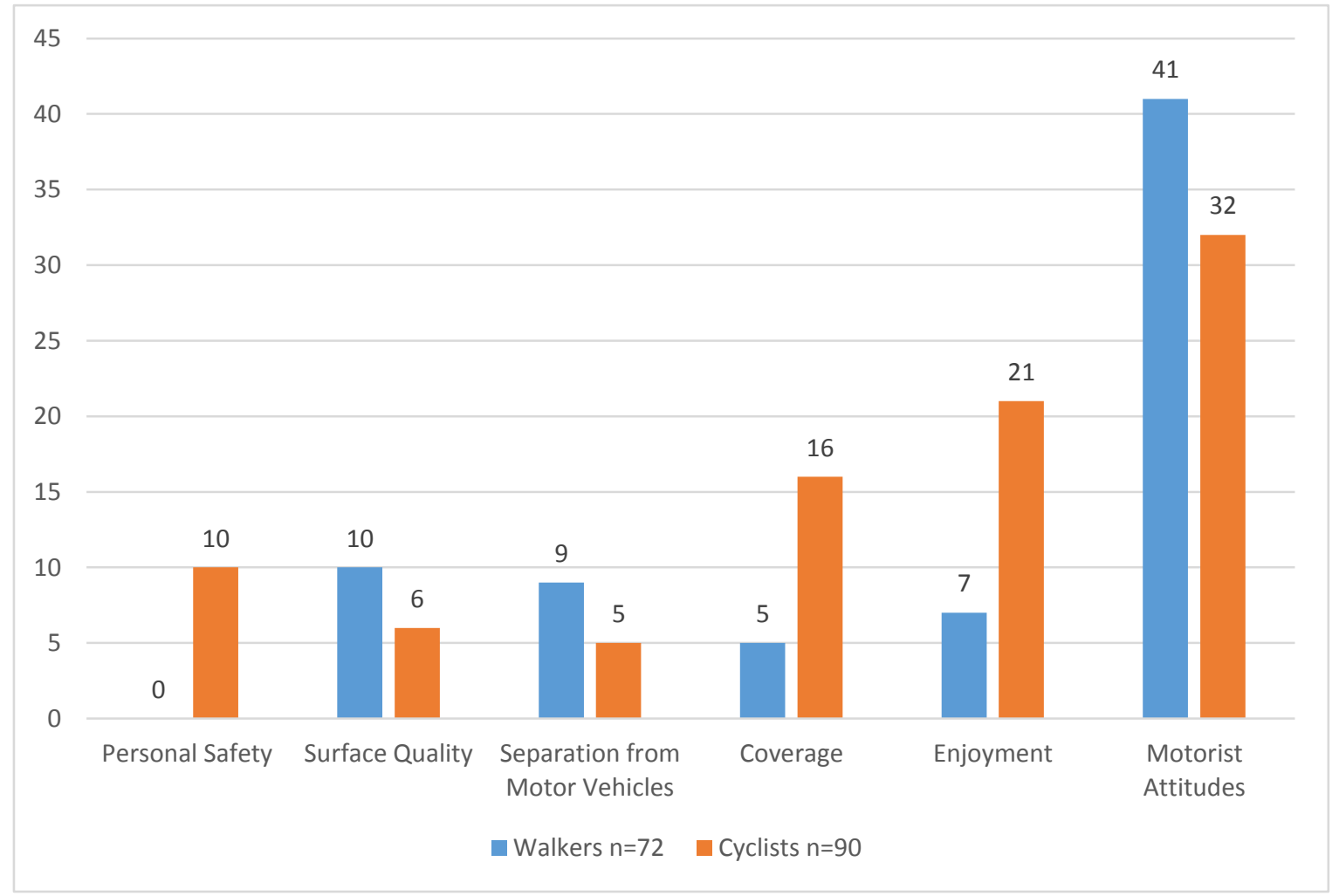

Figure 51: Frequency of \#6 rankings for walkers per criterion in 2013

The results of these ratings and rankings are particularly interesting for cyclists in that they clearly call into question the EEM's values for Relative benefits for different types of cycle facilities, which predict that cyclists will only spend marginally more time on separated pathways than they would on roads marked with cycle lanes. It is important to note that these intercept surveys took place on separated shared pathways; it may be that users of such facilities would hold a higher preference towards separated cycling facilities than the average cyclists. However, one could make the same argument in regard to walkers; but the results for them seem to suggest that they are seldom concerned about facilities that are separated from motor vehicle traffic. Furthermore, these results support numerous studies' findings that there are large portions of the population who are not willing to cycle on on-road facilities that place them in close proximity to motor vehicle traffic (Forsyth \& Krizek, 2010; Hunt \& Abraham, 2006; Kingham et al., 2011; Ogilvie et al., 2010; Pucher \& Buehler, 2008; M Yeates, 2001). As mentioned before, the intercept survey found results suggesting that nearly one third of regular cyclists intercepted in 2013 were living in Hastings and New Plymouth, but were not regular cyclists prior to the intervention.

Research from Ogilvie et al. (2010) suggests that separated bicycle facilities are an essential component to encourage young, inexperienced and/or less confident cyclists to 
become regular cyclists. Garrard et al. (2008) argue that low rates of cycling amongst women in several developed countries are likely due to lack of available facilities separated from motor vehicle traffic. A 2003 study by Garrard cites data suggesting that Denmark, the Netherlands and Germany, all of whom have invested heavily in separated bicycle facilities, also have higher rates of female cycling than males. A few users explicitly mentioned that they became regular cyclists as a result of the separated shared pathways built as part of the MCP interventions; these included respondent \#99, a female in her 60s living in Hastings, who regularly cycles with her neighbour; and respondent \#139 who is a male in his 60s who suffered a stroke several years ago and no longer has a driver's license, but is able to cycle around Hastings in the safety of the $i$ Way facilities. Respondent \#104 from Hastings argued that cyclists are safer off roads, and the separated pathways have facilitated an "unreal change in cycling culture".

Both walkers and cyclists placed high values on walking and cycling facilities in Hastings and New Plymouth. Almost 75\% of walkers valued these facilities at over NZ\$200 per year, with $42 \%$ valuing them higher than NZ $\$ 500$. As for cyclists, nearly $85 \%$ of them valued the facilities at over $\mathrm{NZ} \$ 200$, with $58 \%$ of respondents valuing them at more than NZ\$ 500. These values were high for both users, and especially for cyclists. The distributions suggests that a significant number of users might have even valued these facilities at greater than NZ\$1,000 (the survey did not seek values this high).

\subsubsection{Community related benefits}

A significant number of users suggested that the intervention improved accessibility for various groups including children, the elderly, and the disabled. Respondent \#12 in New Plymouth, along with several others, noted the increasing numbers of school children walking and cycling to school, an activity that has been in decline across many developed countries (Chapman et al., 2014; Ministry of Transport, 2009; Whitzman et al., 2010). Respondent \#29 suggested that all users in New Plymouth have easy access to great scenery and respondent \#79 added that "few cities have such nice places to walk". Several comments were made regarding increased community cohesion including respondent \#18 from New Plymouth boasting that they regularly recognise friends, or even other regular users on their new or improved facilities. Respondent \#54 from New Plymouth stated that the walking and cycling facilities are "a great source of community pride”; on a similar note, respondent \#26 suggested that these facilities in New Plymouth promote active lifestyles and further stated that, accordingly, "people want to live here". 


\subsubsection{Key reservations about the Model Communities Programme}

The project managers themselves provided some of the most insightful statements in reference to weaknesses of the MCP. In Chapter 8, the Hastings Project Manager noted NZTA's lack of flexibility in funding traffic calming infrastructure, especially pedestrian crossings. User perceptions of walking facilities noted in Chapter 7 did not improve significantly, and this may at least be partially attributed to the fact that the MCP was not able to modify roads in order to increase the appeal of walking in the city centres. Respondent \#130 remarked how despite all the efforts of the MCP to improve walking and cycling facilities in Hastings, he still found the shopping in the Hastings CBD as a pedestrian to be an unpleasant experience - implicitly because of road traffic. The New Plymouth Project Manager noted that he had had little success to date changing perceptions of the need for so much parking within the CBD. Interestingly enough, during this author's visit to the New Plymouth CBD, numerous signs marking crossings on Devon St (in the main retail and hospitality centre) requested pedestrians to yield to motor vehicles, as seen on the cover page of this study. This bias towards motor vehicles in the city centre may partially explain why pedestrians only reported a minor improvement in walking facilities in both Hastings and New Plymouth. The Hastings Project Manager added that the conditions NZTA placed on their portion of the MCP funding limited how they were able to improve walking facilities on NZTA controlled roads, notably in respect to traffic calming facilities such as pedestrian crosswalks.

As mentioned in Chapter 8, both MCP project managers expected to regularly work with local District Health Boards in promotion of active travel in their districts. Lack of alignment over the strategic goals of the project effectively limited any meaningful collaboration between these parties. In Australia, a study by Cole, Burke, Leslie, Donald, \& Owen (2010) found that these types of inter-agency issues in relation to implementing effective active transport policy is common, and city agencies' limitations in terms of resources, technical expertise and conflicting institutional cultures were the primary detriments to these potentially effective partnerships. This issue in particular should be noted by project managers for future interventions of a similar nature to the MCP.

\subsubsection{Key criticisms of the CBA framework}

Project managers were given the opportunity to evaluate the limitations of CBA as an assessment tool for evaluating the effectiveness of an intervention. Both project managers 
suggested several telling limitations of a CBA approach, most of which have frequently been identified in academic literature.

As noted earlier, the Hastings Project Manager stated that traditional CBA does not adequately capture many community benefits inherent to walking and cycling interventions. Such benefits include providing an environment which a child is safe to explore and to develop healthy habits from an early age. These ideas are also heavily discussed within UNICEF's Childfriendly Cities Initiative (2009) as well as by Whitzman et al. (2010). In addition to this, the Hastings Project Manager also argued that CBA has long undervalued, and continues to undervalue, the external costs to society of motor vehicle transport, an argument highlighted by numerous academics and institutions including a report by the World Bank entitled The Social Cost of Transport (Ruta, 2002), a report by the World Health Organization entitled Transport, Environment and Health (Dora et al., 2000) and in a Victoria Transport Policy Institute report entitled Transportation Market Distortions (Litman, 2006).

The New Plymouth Project Manager listed many benefits of active travel facilities that economic evaluation frameworks consistently overlook, often echoing the concerns of transportation expert Todd Litman, including: increased mobility for children which decreases a parent's chauffeuring burden (Litman, 2013a), improved social equity and accessibility for transport (Litman, 1998), improved social cohesion from an environment that encourages social interaction (Litman, 2004) and greater potential for tourism (Litman, 2013a). The New Plymouth Project Manager also mentioned that creating a more desirable place as a result of investing in walking and cycling facilities can attract potential employers as well as skilled workers to settle in New Plymouth. This author is unaware of any study that directly investigates this proposition, although facilities for active travel are sometimes mentioned in terms of competitive, liveable cities (Puentes, 2009). This notion is a nonetheless plausible if untested theory.

\subsection{Strengths and limitations of this research}

A key theme of this study has been the uncertainties surrounding any evaluation of active travel interventions, consistent with the conclusions of several studies (Saelensminde, 2004; SQW, 2007; Wilson \& Cope, 2011). This section focuses on strengths and 
limitations of this study, including those relating to uncertainties in the economic evaluation and mixed methods components of the study.

\subsubsection{Analysis of potential limitations of the economic evaluation}

Economic evaluation of walking and cycling interventions, centred around data from census and household travel surveys, requires making numerous assumptions that bring some level of ambiguity into the final results. Ogilvie et al. (2011), Rietveld (2000) and Saelens et al. (2003) highlight the limitations of walking and cycling data in providing accurate indications of wider walking and cycling trends, and the same applies in this study, when assumptions are made concerning baseline (or control city) trends. Krizek et al. (2006) note that cycling's minor overall share compared to other transport modes leaves it susceptible to higher levels of sampling error, as has been reported concerning NZHTS data prior to 2008.

The economic evaluation approach used in this study required making several other assumptions affecting estimation of trips for non-work purposes, the number of regular mode users, yearly days walked/cycled and average trip distance. The NZHTS provided data that allowed for reasonable estimation of average trip distances as well as numbers for "other trips". One study (Goodman et al., 2013) estimated that walking and cycling trips for purposes other than work grew faster as a result of an improvement in walking and cycling facilities. The present study, however, assumed that trips for other purposes grow at the same rate as reported census trips to work, likely representing a conservative estimate. It is also worth noting that there are uncertainties into how cycling growth will change in the post-intervention period. To this author's knowledge, there are no robust models that are known to accurately forecast cycling growth in the response to a medium to large-scale intervention. The cycling growth figures used for this study are untested, but realistic estimates. To estimate the number of regular walkers and cyclists, the present study also used a formula provided in a report by Krizek et al. (2006). This author is unaware of a similar formula that would potentially better represent walking and cycling in the New Zealand context. In using Krizek's approach, this author acknowledges that this study has nonetheless been critical of NZTA's decision to use Krizek et al.'s (2006) stated preference results for the EEM's Relative benefits for different types of cycle facilities. This could be construed as inconsistent. However, the aforementioned formula for predicting regular cyclist numbers from Census data has 
been found to be an accurate estimator for regular cyclists for the majority of US cities where the formula was tested. Results from the adapted stated preference survey (the basis for the EEM's Relative benefits values) were not reported to have been tested to see if they reflect similar relative benefit values in other cities. This author acknowledges the potential value of undertaking future research to adapt these estimations to reflect the urban New Zealand context.

Another limitation of this study is that it does not account for walkers whose preferences now favour cycling trips as a result of an intervention, an issue highlighted by Ogilvie et al. (2004). Given the reduction in walking trips to work demonstrated in census data, and given the advent of better cycling facilities in the intervention cities, it is likely that at least some of the surveyed walkers in the 2006 census were cycling by the 2013 census. Although these cyclists would still benefit from switching modes, arguably they would benefit less than would a new active travel participant, that is, someone switching from a comparatively sedentary mode, including private car use. The health benefits from an inactive individual becoming active are significantly higher than a previously active individual becoming marginally more active. Also, there are arguably no congestion savings from a walker switching to cycling.

\subsubsection{Analysis of potential strengths of the economic evaluation}

Blanco et al. (2009) argue that past economic evaluation of active travel interventions often lacked robust research designs using baseline data and control groups. This study compared the intervention cities to two control cities sharing similar geographical and demographic features to their respective intervention city. The use of a controlled study design helps establish causality, allowing for observed net increases (i.e. intervention city less control city effects) in active travel to be calculated as benefits. This research did so by estimating the number of walking and cycling users and trips that would likely have been lost in the absence of the intervention.

As mentioned earlier in this chapter, the BCRs estimated using the quasi-experimental methodology were over twice as large as the BCRs calculated from the observed changes in active travel estimated from the intervention alone. This methodology faces the criticism that it potentially ignores other factors contributing to the decline of walking and cycling in the control cities. One such factor is population dynamics. The population increased by $0.4 \%$ in the control cities in comparison to over $5 \%$ in the intervention cities, 
and this may explain some of the difference in outcomes, rather than the intervention. It is also worth mentioning that the population of the intervention cities $(\sim 147,000$ residents) is around twice the size of the population of the control cities $(\sim 66,000$ residents). However, it is not clear why population growth (or population size) in itself would alter mode shares significantly in the absence of other influences.

Krizek et al. (2006) argue that cities or even different neighbourhoods in the same city rarely share the same characteristics that make places desirable to cycle; and sociodemographic and geographic characteristics could be important influences on active travel. Thus evaluators expecting one place to be a valid control for an intervention in another place are potentially being optimistic. However, this author argues that within this study, evaluating the intervention cities relative to the conditions of the control cities at the very least provides a reasonably realistic assessment of potential benefits, in terms of trips 'saved', taking account of a counterfactual of diminishing rates of walking and cycling. It is also important to note the geographical and demographic similarities between the intervention cities and their respective control cities. However, there are irreducible uncertainties associated with this methodology and it may be argued that an economic evaluation measuring active travel changes arising from the intervention alone is a more robust methodology.

\subsubsection{Limitations of the mixed methods analysis}

There are some limitations to the intercept survey data that merit discussion. First, sampling was limited to separated shared pathways; thus, the sample pool was not, and was not intended to be, a representative sample of all walkers and cyclists in Hastings and New Plymouth. It was simply designed to evaluate the perceptions of the users of these separated pathways as to benefits arising from the MCP intervention. The intercept survey also offers some interesting data regarding average walking and cycling distances travelled, before and after the intervention, and hence changes over time. However, this survey is not able to use these data to determine conclusively whether users are travelling further or whether more users are accessing the infrastructure. This is because this survey design does not sample those users who were walking/cycling before the intervention but who are no longer walking/cycling. Only a comprehensive repeated sample can give such a picture, which is why the census data provided a valuable additional insight into background trends. 
It is also worth mentioning that data provided by respondents to the intercept survey may not reflect the reality of their travel habits, but rather their perceptions of their travel habits, thus lending the data some degree of bias and uncertainty. It is also possible that bias may arise from the lack of respondents who may have given up active travel after a period of engaging in it. Likewise, feedback provided by the district managers may be limited to the observations they are willing to allow to go on the public record. Nevertheless the feedback provided by both groups of stakeholders proved to be a rich source of insights.

\subsubsection{Strengths of the mixed methods analysis}

Economic evaluations of active travel interventions rarely delve deeply into analysing nonquantifiable and non-monetisable benefits, although one study (SQW, 2007) suggests that multi-criteria analysis and other qualitative methodologies are desirable when evaluating the merits of an intervention in the UK. This research uses a mixed methods analysis in the form of an intercept survey and project manager interviews with the goals of achieving a more holistic and comprehensive analysis of MCP costs and benefits. The author is unaware of other studies taking this approach, or of any other active travel intervention that has been evaluated using conventional CBA complemented with user feedback from intercept surveys.

This mixed methods analysis has provided a unique opportunity to appraise an active travel intervention in a manner that addresses existing academic literature's criticism of previous evaluations, while still providing a CBA benefit-cost ratio, described by Glaister (1999) as the most useful component for policy makers' comparison of proposed transportation projects. More importantly, the mixed methods approach has allowed for users of this infrastructure to have a voice through giving feedback and expressing their perceptions of benefits for the MCP. This feedback is largely ignored under traditional economic evaluation frameworks. 


\subsection{Policy conclusions}

A few conclusions on desirable policy change in regard to NZTA's treatment of walking and cycling in its land transport investment appraisal guidance can be derived from this study.

1) Re-evaluation of the EEM's treatment of benefits for walking and cycling.

Although the 2013 EEM provides an arguably reasonable benefit for health, the benefits associated with safety and road traffic reduction are substantially undervalued, and those related to improved community outcomes are barely considered. Further, if it is national transportation authorities' desire to promote greater participation in active travel, improved social and inter-generational equity outcomes and to reduce land transportation's contribution to greenhouse gas emissions, NZTA should adopt a social discounting scheme that appropriately reflects social factors in valuation and in discounting over time investments in active travel vis à vis other investments.

\section{2) Re-evaluation of the EEM's Relative benefits for different types of cycle facilities}

The methodology of this calculation is not of concern, but the fact that the values provided reflect a US study whose conditions are very different from that of the general New Zealand population suggests that it is an inappropriate representation of cyclists' preferences in New Zealand. The use of this approach may partially explain the reluctance of transportation authorities in New Zealand to invest in separated bicycle facilities. Values presented in a "Relative benefits" table should reflect cyclists' actual preferences for types of cycling facilities in the urban New Zealand context.

Discriminating values should be provided for separated cycle pathways as cyclists will have different preferences for those pathways that are adjacent to busy arterial roads compared to those that are out of sight of traffic and likely have greater scenic and aesthetic qualities. 
3) Provide a clearer methodology for the EEM's treatment of walking and cycling facilities.

The current 2013 EEM does not reference its walking and cycling section. The 2010 EEM Volume 2 does provide these references, although in some cases it provides incorrect references (NZ Transport Agency, 2010, p.8-6). Local, regional and national transportation authorities should be allowed to follow transparent frameworks where they themselves can determine whether these methodologies are likely to reflect their contextual realities. Further, it would be desirable to unpack the composite benefits estimation to make it clearer how benefits are being calculated. Absence of such transparency could otherwise be taken as an agency's bias for or against certain modes of transport.

4) Continue the Model Communities Programme to other cities

A report by NZTA entitled "The Walking and Cycling Model Community Story” (2013b) gives general praise to the MCP in Hastings and New Plymouth. Data from census and NZHTS supports the agency's positive evaluation of this programme, notably in regard to reversing decades of declining cycling rates. The end of NZTA's report states that:

It is unlikely that there will be more branded model communities, as such, but the model communities experience has demonstrated that with a modest targeted investment agreed community outcomes can be delivered. (NZTA, 2013b, p.63)

In recognizing the programme's successes, NZTA simultaneously signals its demise. 20 cities applied but were not made Model Communities (Dance, 2012), suggesting that a large number of cities wish to improve walking and cycling conditions, but are less likely to do so in the absence of national funding. The United Nations Environment Programme notes that "political commitment to cycling as a low-cost mode of transport" was largely responsible for reversing declining rates of cycling in Denmark and the Netherlands (2010, p.19). Both of these countries are widely considered model cases for large-scale uptake of utilitarian cycling. 
NZTA does state that it is receptive to applications from other councils wishing to provide similar interventions (NZTA, 2013b); however one could argue that this passive approach to investing in improved walking and cycling facilities is unlikely to reverse declining rates of walking and cycling, notably within smaller cities in New Zealand.

5) Allow its economic evaluative framework to promote attractive urban areas to walk, where pedestrian needs are prioritised over motor vehicles'.

MCP project manager feedback reporting difficulty in building pedestrian crossings on NZTA controlled roadways suggests that NZTA is likely putting the needs of motorists ahead of pedestrians. Future medium to large scale active travel interventions should ease these strict policies to see how they might affect transportation habits in urban areas. Past and current rates of decline in walking in the intervention cities according to the census and NZHTS data suggests that the MCP may not have improved walkability enough to promote wider use of walking facilities.

\subsection{Recommendations for further research}

Active transport in the New Zealand context seems to be a relatively "untapped" field of study, with a multitude of topics in need of research. The following are a few pressing topics, better understanding of which would have aided the research process of this study.

1) In the urban New Zealand context, to what extent do interventions that promote cycling motivate walkers to change modes?

2) What are cyclists' perceptions of relative benefits for different types of cycle facilities within the urban New Zealand context? How might level of service for cyclists be maximised? 
3) What are the most effective means of promoting walking in small cities in New Zealand?

4) What are the most effective strategies for promoting walking and cycling by individuals from communities of lower socio-economic status in New Zealand?

5) To what extent do increasing numbers of walking and cycling trips replace motor vehicle trips in urban areas in New Zealand?

6) To what extent do negative perceptions of safety prevent potential cyclists from riding bicycles for everyday trips such as travel to work?

7) How can Census travel to work data be interpreted to represent everyday walkers and cyclists traveling for all purposes? 


\section{Chapter 10 - Conclusion}

This study set out to evaluate the effectiveness of investment in active travel interventions to boost walking and cycling rates in the urban New Zealand context using NZTA's Model Communities Programme as a case study. This study has developed a unique and comprehensive framework for evaluation which complements traditional economic evaluation with mixed methods analysis utilising feedback from both MCP users and project managers to offer a more holistic analysis of the intervention's successes and shortcomings.

Economic evaluation of the MCP in the form of CBA has shown that the intervention is likely to be cost-beneficial (benefits exceed costs) under even conservative evaluative frameworks. When allowing for a comprehensive analysis of potential benefits, the evaluation calculated benefit to cost ratios (BCRs) ranging from 5.3 to 10.3 , with a strong argument that a medium estimate of 5.3 is not only reasonable, but calculated under conservative conditions for a variety of likely benefits. However, this figure largely excludes the potential benefits associated with improved community outcomes. Were these to be monetised and included, the BCRs would in principle be greater. No robust framework known to the author is currently capable of quantifying these.

The most robust evaluation BCR value of 5.3 for the intervention is many-fold higher than the BCR calculated using NZTA's 2013 EEM framework (BCR of 1.7), suggesting that NZTA's current practice of evaluating walking and cycling is likely to significantly underestimate the potential benefits associated with increased uptake in walking and cycling. Also of particular interest to this topic, this study has found the EEM's approach to assessing Relative benefits for different types of cycle facilities is flawed and should be redrawn using data relevant to cyclists' preferences in urban New Zealand.

Furthermore, when accounting for the number of walking and cycling trips saved - given the counterfactual of declining rates of walking and cycling across small cities in New Zealand - by calculating the BCRs using a quasi-experimental design, estimated BCRs were at least twice as high in comparison to the original calculations for the intervention itself. The quasi-experimental model accounts for what would likely have happened in the absence of the intervention, making the generally reasonable assumption that the control cities give a fair picture of this. However, the quasi-experimental model may not fully account for the complex factors causing rates of walking and cycling to differ between locations (Krizek et al., 2006), and thus should be used with caution, reflecting the 
uncertainty in the estimates. At the very least, the quasi-experimental calculations in this study can provide valuable insight into the potential benefits that can be achieved in areas where active travel is otherwise likely to be in decline.

The use of Census data to generate these CBA estimates is recognised to involve uncertainties given the small window of analysis provided by these data; however trends identified within the Census data are similar to those identified using NZHTS weighted data. Thus the author is confident that these background conditions of evaluation reflect the best available data at the time this analysis was performed.

User feedback from the intercept surveys showed several positive trends. Walkers reported a modest increase in average distances walked per week compared to baseline distances walked. Walkers' perceptions of the quality of their facilities showed modest gains after the intervention, although it is worth noting their pre-intervention ratings were already quite high. Walkers surveyed ranked factors of "personal safety" and "enjoyment" as most important to them.

Cyclists reported lower mean average weekly distances cycled after the intervention. However, limiting the analysis to cyclists who were regularly cycling pre and postintervention, these cyclists reported higher mean weekly distances. This would suggest that new cyclists are riding shorter average weekly distances than the baseline groups of cyclists who are likely more experienced. Cyclists' baseline ratings of cycling facilities were significantly lower than walkers' baseline ratings; however cyclists' ratings increased to a greater extent at the post-intervention stage when compared to the change in ratings for walkers. This may suggest that the level of service has increased significantly more for cyclists than for walkers. However, it is worth noting that cyclists' post-intervention ratings are still lower than walkers' post-intervention ratings, suggesting that cycling facilities' level of service still has room for improvement.

Walkers and cyclists placed high values on the facilities in question, with nearly $52 \%$ valuing the facilities at more than NZ\$500, although cyclists seemed to place a higher value on their facilities than walkers. Willingness-to-pay values must, however, be treated with caution because of the known problems with such valuations.

Feedback from MCP project managers was generally positive with a few notable points of critique. Both project managers highlighted the comparatively higher enthusiasm for the intervention amongst communities and schools of higher socio-economic status, a trend also noted by Ogilvie et al. (2010) in other studies. Both project managers suggested 
targeting educational and promotional activities to communities and schools from higher deprivation areas to encourage higher numbers of these individuals to take up regular walking and cycling. One project manager also noted that it is prohibitively difficult to set up pedestrian crossings on routes monitored by NZTA. This arguably reflects NZTA's predisposition towards motor vehicle transport rather than active travel modes.

Whether appraising the intervention from an economic standpoint or using feedback from MCP users and project managers, the intervention package - i.e. the combination of infrastructure investment and education/promotion - by and large appears to have been effective. Although walking rates still appear to be in decline in the intervention cities, they are more than made up for by large increases in cycling. Data from the censuses and the NZHTS suggest cycling has generally been in decline for decades, notably for small cities. NZTA's decision not to formally extend the MCP to other district councils is disappointing given the conclusions reached here, and NZTA's own perceptions of the intervention's success (NZTA, 2013b).

Although NZTA states it is open to funding requests for similar interventions in other areas (2013b), its approach to promoting active travel to other areas of New Zealand should be construed as passive at best. Without strong policy actively encouraging walking and cycling at a national level, it is unlikely that New Zealand will achieve widespread gains in active travel within the near future, despite the benefits to health, the environment and the community that such increases can yield. In the absence of such investment, the conclusions of this study suggest that walking and cycling rates in small cities across New Zealand stand to lose the most. 


\section{References}

Ackerman, F., \& Stanton, E. A. (2010). The social cost of carbon. Economics for Equity and Environment (E3 Network). Available Online at Http://www. e3network. org/papers/SocialCostOfCarbon_SEI_20100401. Pdf. Retrieved from http://environmentaleconomics.net/papers/SocialCostOfCarbon_SEI_20100401.pdf

Andersen, L., Schnohr, P., Schroll, M., \& Hein, H. (2000). All-Cause Mortality Associated With Physical Activity During Leisure Time, Work, Sports, and Cycling to Work. Internal Medecine, 160, 1621-1628.

Banister, C., Turner, J., Richardson, T., \& Rees Jeffreys Road Fund. (2000). Unravelling the transport web: delivering the new transport realism in a complex policy environment : a report on the wider policy conflicts that exist in the transport “web.” London: Landor.

Banister, Chris, \& Gallent, N. (1999). Sustainable communting: A contradiction in terms? Regional Studies, 33(3), 274-280.

Bateman, I., Turner, R., \& Bateman, S. (2005). EXTENDING COST BENEFIT ANALYSIS OF UK HIGHWAY PROPOSALS: ENVIRONMENTAL EVALUATION AND EQUITY. Centre for Social and Economic Research on the Global Environment.

Bauman, A., Rissel, C., Garrard, J., Ker, I., Speidel, R., \& Fishman, E. (2007, August). Barriers, facilitators and interventions to get more Australians physically active through cycling. Cycling Promotion Fund.

Beetham, J. (2014). Re-Cycling the Streets: Exploring the Allocation of Public Space for Transport. Victoria University of Wellington.

Beeton, S. (2003). An economic analysis of rail trails in Victoria, Australia. Research Monograph (Bendigo, VIC: La Trobe University, School of Sport, Tourism and 
Hospitality Management). Retrieved from

http://www.yooyahcloud.com/CYCLETOURISMAUSTRALIA/yCwtDb/Beeton _MTM_Rail_Trail.pdf

Beierle, H. (2011). Bicycle Tourism As A Rural Economic Development Vehicle. Department of Planning, Public Policy \& Management, University of Oregon. Retrieved from https://scholarsbank.uoregon.edu/xmlui/handle/1794/11679

Blanco, H., Alberti, M., Forsyth, A., Krizek, K. J., Rodríguez, D. A., Talen, E., \& Ellis, C. (2009). Hot, congested, crowded and diverse: Emerging research agendas in planning. Progress in Planning, 71(4), 153-205. doi:10.1016/j.progress.2009.03.001

Boardman, A., Moore, M., \& Vining, A. (2010). The Social Discount Rate for Canada Based on Future Growth in Consumption. Canadian Public Policy, 36(3), 325343.

Börjesson, M., \& Eliasson, J. (2012). The value of time and external benefits in bicycle appraisal. Transportation Research Part A: Policy and Practice, 46(4), 673-683. doi:10.1016/j.tra.2012.01.006

Boyce, C. (2010). Walkability, social inclusion and social isolation and street redesign. Built Environment, 36(4), 461-473.

Bryman, A. (2008). Social Research Methods (3rd ed.). Oxford: Oxford University Press. Cavill, N., Kahlmeier, S., Rutter, H., Racioppi, F., \& Oja, P. (2008). Economic analyses of transport infrastructure and policies including health effects related to cycling and walking: A systematic review. Transport Policy, 15(5), 291-304. doi:10.1016/j.tranpol.2008.11.001

Cervero, R. (2011). Beyond Travel Time Savings: An Expanded Framework for Evaluating Urban Transport Projects (Transport Research Support). Washington, D.C.: The International Bank for Reconstruction and Dvevelopment / The 
World Bank. Retrieved from

http://siteresources.worldbank.org/INTTRANSPORT/Resources/336291-

1239112757744/5997693-1294344242332/Traveltimesaving.pdf

Chapman, R., Howden-Chapman, P., Keall, M., Witten, K., Abrahamse, W.,

Muggeridge, D., ... Grams, M. (2014). Increasing active travel: aims, methods and baseline measures of a quasi-experimental study in New Zealand.

Cole, R., Burke, M., Leslie, E., Donald, M., \& Owen, N. (2010). Perceptions of representatives of public, private, and community sector institutions of the barriers and enablers for physically active transport. Transport Policy, 17(6), 496-504. doi:10.1016/j.tranpol.2010.05.003

Conn, V. S., Hafdahl, A. R., Cooper, P. S., Brown, L. M., \& Lusk, S. L. (2009). MetaAnalysis of Workplace Physical Activity Interventions. American Journal of Preventive Medicine, 37(4), 330-339. doi:10.1016/j.amepre.2009.06.008

Craig, P. (2013). The Other 25\%: The Big Move and Active Transport Investment. Toronto: Clean Air Partnership. Retrieved from http://www.tcat.ca/sites/all/files/TCAT-MX_FINAL.pdf

Creswell, J., \& Plano Clark, V. (2011). Designing and conducting mixed methods research (2nd ed.). Thousand Oaks, California: Sage Publications.

Creswell, J. W., Klassen, A. C., Plano Clark, V. L., \& Smith, K. C. (2011). Best practices for mixed methods research in the health sciences. Bethesda (Maryland): National Institutes of Health. Retrieved from https://tigger.uic.edu/jaddams/college/business_office/Research/Best_Practices_fo r_Mixed_Methods_Research.pdf

Daley, M., Rissel, C., \& Lloyd, B. (2007). All Dressed Up and Nowhere to Go?: A Qualitative Research Study of the Barriers and Enablers to Cycling in Inner 
Sydney. Road \& Transport Research: A Journal of Australian and New Zealand Research and Practice, 16(4), 42.

Damart, S., \& Roy, B. (2009). The uses of cost-benefit analysis in public transportation decision-making in France. Transport Policy, 16(4), 200-212. doi:10.1016/j.tranpol.2009.06.002

Dance, G. (2012). Model Community Journey making walking and cycling the easier, safer transport choice (NZTA). Presented at the IPENZ Central Region, August 15th, 2012.

Dekoster, J., Schollaert, U., European Commission, \& Directorate-General for Environment, N. S., and Civil Protection. (1999). Cycling: the way ahead for towns and cities. Luxembourg: Office for Official Publications of the European Commission.

Department for Transport. (2012). The Greenhouse Gases Sub-Objective (No. TAG Unit 3.3.5). London. Retrieved from http://www.dft.gov.uk/webtag/documents/expert/pdf/U3_3_5-ghg-120723.pdf

Dietz, S., Anderson, D., Stern, N. H., Taylor, C., \& Zenghelis, D. (2007). Right for the right reasons. World Economics, 8(2), 229-258. London. Retrieved from http:/www.lse.ac.uk/GranthamInstitute/wp-content/uploads/2014/06/WorkingPaper-180-Dietz-and-Stern-2014.pdf

Dietz, S., Stern, N. (2014). Endogenous growth, convexity of damages and climate risk: how Nordhaus' framework supports deep cuts in carbon emissions. Centre for Climate Change, Ecomomics and Policy

Dora, C., Phillips, M. A., World Health Organization, \& Regional Office for Europe. (2000). Transport, environment, and health. Copenhagen: World Health Organization, Regional Office for Europe. Retrieved from http://site.ebrary.com/id/10214514

Elliott, J. (2005). Using Narrative in Social Research (1st ed.). London: Sage Publications. 
Environmental Protection Agency. (2011). Greenhouse Gas Emissions from a Typical Passenger Vehicle. Ann Arbor. Retrieved from http://www.epa.gov/otaq/climate/documents/420f11041.pdf

Forsyth, A., \& Krizek, K. J. (2010). Promoting walking and bicycling: assessing the evidence to assist planners. Built Environment, 36(4), 429-446.

Frank, L. D. (2004). Economic determinants of urban form. American Journal of Preventive Medicine, 27(3), 146-153. doi:10.1016/j.amepre.2004.06.018

Frank, L. D., \& Engelke, P. O. (2001). The Built Environment and Human Activity Patterns: Exploring the Impacts of Urban Form on Public Health. Journal of Planning Literature, 16(2), 202-218. doi:10.1177/08854120122093339

Garrard, J. (2003). Healthy revolutions: promoting cycling among women. Health Promotion Journal of Australia, 14(3), 213-215.

Garrard, J., Rose, G., \& Lo, S. K. (2008). Promoting transportation cycling for women: The role of bicycle infrastructure. Preventive Medicine, 46(1), 55-59. doi:10.1016/j.ypmed.2007.07.010

Genter, J. A., \& NZ Transport Agency. (2009). Valuing the health benefits of active transport modes. Wellington, N.Z.: NZ Transport Agency.

Giles-Corti, B., Foster, S., Shilton, T., \& Falconer, R. (2010). The co-benefits for health of investing in active transportation. New South Wales Public Health Bulletin, 21(6), 122. doi:10.1071/NB10027

Glaister, S. (1999). Observations on the new approach to the appraisal of road projects. Journal of Transport Economics and Policy, 227-234.

Glasgow, R. E. (2013). What Does It Mean to Be Pragmatic? Pragmatic Methods, Measures, and Models to Facilitate Research Translation. Health Education \& Behavior, 40(3), 257-265. doi:10.1177/1090198113486805 
Goodman, A., Sahlquist, S., \& Ogilvie, D. (2013). Who uses new walking and cycling infrastructure and how? Longitudinal results from the UK iConnect study. Preventive Medicine, 57(5), 518-524. doi:10.1016/j.ypmed.2013.07.007

Gotschi, T. (2011). Costs and benefits of bicycling investments in Portland, Oregon. Journal of Physical Activity \& Health, 8. Retrieved from http://search.ebscohost.com/login.aspx?direct=true\&profile=ehost\&scope=site\&au thtype $=$ crawler\&jrnl $=15433080 \& \mathrm{AN}=58478880 \& \mathrm{~h}=$ Z6qadd6v9AwXdrMoTDJ\%2 F3IseYzWAD\%2BsMQoQ3Zvuj8ggdY2I8zdmGJUmfOk8oVbxyBrPDbulrIvPg1 $\% 2 \mathrm{FdWrTMiOg} \% 3 \mathrm{D} \% 3 \mathrm{D} \& \mathrm{crl}=\mathrm{c}$

Guion, L. A., Diehl, D. C., \& McDonald, D. (2011). Triangulation: Establishing the validity of qualitative studies. Retrieved from http://edis.ifas.ufl.edu/fy394

Harwatt, H., Jopson, A., Muir, H., Page, M., \& Tight, M. (2010). PERSONAL FACTORS INFLUENCING WALKING AND CYCLING IN URBAN AREAS. 12th World Conference on Transport Research.

Hopkinson, P., \& Wardman, M. (1996). Evaluating the demand for new cycle facilities. Transport Policy, 3(4), 241-249.

Hunt, J. D., \& Abraham, J. E. (2006). Influences on bicycle use. Transportation, 34(4), 453-470. doi:10.1007/s11116-006-9109-1

Intergovernmental Panel on Climate Change. (2014). IPCC Press Release 31 March 2014. Retrieved from http://www.ipcc.ch/pdf/ar5/pr_wg2/140330_pr_wgII_spm_en.pdf

Jacobs, J. (1961). The Death and Life of Great American Cities. New York: Random House.

Jacobsen, P. (2003). Safety in Numbers: more walkers and bicyclists, safer walking and bicycling. Review of the Injury Prevention Editor's Choice Article. 
Joh, K., Nguyen, M. T., \& Boarnet, M. G. (2011). Can Built and Social Environmental Factors Encourage Walking among Individuals with Negative Walking Attitudes? Journal of Planning Education and Research, 32(2), 219-236. doi:10.1177/0739456X11427914

Kahlmeier, S., World Health Organization, \& The World Health Organization Regional Office for Europe. (2013). Health economic assessment tools (HEAT) for walking and for cycling: methodology and user guide : economic assessment of transport infrastructure and policies. Copenhagen: World Health Organisation, Regional Office for Europe.

Keall, M., Chapman, R., \& Howden-Chapman, P. (2010). Changes in frequency of walking and cycling trips in New Zealand: Potential influences of urban form. In Sizing up the City: Urban form and transport in New Zealand. Wellington: Steele Roberts.

Kingham, S., Taylor, K., Koorey, G. F., \& NZ Transport Agency. (2011). Assessment of the type of cycling infrastructure required to attract new cyclists. Wellington, N.Z.: NZ Transport Agency.

Krizek, K. J., United States, Federal Highway Administration, National Research Council (U.S.), Transportation Research Board, National Cooperative Highway Research Program, \& American Association of State Highway and Transportation Officials. (2006). Guidelines for analysis of investments in bicycle facilities. Washington, D.C.: Transportation Research Board.

Leyden, K. (2003). Social Capital and the Built Environment: The Importance of Walkable Neighborhoods. Research and Practice, 93(9), 1546-1551.

Litman, T. (1998). Whose Roads. Victoria Transport Policy Institute (www. Vtpi. Org). Retrieved from http://www.islandnet.com/ litman/whoserd.pdf 
Litman, T. (2004). Economic value of walkability. World Transport Policy \& Practice, $10(1), 5-14$.

Litman, T. (2006). Transportation Market Distortions. Berkeley Planning Journal, 19. Retrieved from http://search.ebscohost.com/login.aspx?direct=true\&profile=ehost\&scope=site\&au thtype $=$ crawler\&jrnl $=10475192 \&$ AN $=25138811 \& \mathrm{~h}=$ Hl4CRYWvx $5 f h h P 5 i Q A 1 r$ r $\%$ 2BVBwo6FF5QfNPbxzUAxCPb\%2B9h3sfpQx6gsaoam1f\%2Fl\%2BJ4HrqZv\%2 BPVXyejbrDvLmkg\%3D\%3D\&crl=c

Litman, T. (2013a). Evaluating Non-Motorized Transportation Benefits and Costs. Victoria Transport Policy Institute.

Litman, T. (2013b). Transportation and Public Health. Annual Review of Public Health, 34(1), 217-233. doi:10.1146/annurev-publhealth-031912-114502

Litman, T. (2013c). Travel Time Costs. Victoria Transport Policy Institute (www. Vtpi. Org).

Macmillan, A. K. (2012). Intervening in the trip to work: a system dynamics approach to commuting and public health. ResearchSpace@ Auckland.

MacMillen, J., Givoni, M., \& Banister, D. (2010). Evaluating active travel: decisionmaking for the sustainable city. Built Environment, 36(4), 519-536.

McClintock, H. (2002). The development of UK cycling policy. In Planning for cycling: principles, practice, and solutions for urban planners. (H McClintock., pp. 1735). US \& Cambridge, UK: CRC Press and Woodhead Publishing.

Mees, P., \& Dodson, J. (2006). Backtracking Auckland: bureaucratic rationality and public preferences in transport planning, April 2006 / Paul Mees, Jago Dodson. Brisbane, Qld.: Urban Research Institute, Griffith University. 
Mills, P., Kessler, R., Cooper, J., \& Sullivan, S. (2007). Impact of a Health Promotion Program on Employee Health Risks and Work Productivity. The Science of Health Promotion, 22(1), 45-53.

Minister of Transport. (2005). Getting there-- on foot, by cycle: a strategy to advance walking and cycling in New Zealand transport. Wellington: Minister of Transport. Ministry for the Environment. (2007). Environment New Zealand 2007: Transport. Wellington. Retrieved from http://www.mfe.govt.nz/publications/ser/enz07dec07/chapter-4.pdf

Ministry for the Environment. (2009). Greenhouse gas emissions and removals.

Wellington. Retrieved from http://www.mfe.govt.nz/environmentalreporting/atmosphere/greenhouse-gases/emissions.html

Ministry for the Environment. (2013). New Zealand's Greenhouse Gas Inventory \& Net Position Report 1990-2011. Wellington. Retrieved from http://www.mfe.govt.nz/publications/climate/greenhouse-gas-inventory-2013snapshot/2013-snapshot.pdf

Ministry of Health. (2010). Mortality and Demographic Data. Retrieved from http://www.health.govt.nz/publication/mortality-and-demographic-data-2010

Ministry of Health. (2012a). Health Behaviours and Risk Factors (Adults). Retrieved from http://www.health.govt.nz/system/files/documents/publications/health-of-newzealand-adults-2011-12-section2.pdf

Ministry of Health. (2012b). Health Behaviours and Risk Factors (Children). Retrieved from http://www.health.govt.nz/system/files/documents/publications/health-ofnew-zealand-child-2011-12-section1.pdf

Ministry of Transport. (2008). The New Zealand Transport Strategy 2008. Retrieved from http://www.transport.govt.nz/assets/Import/Documents/NZTS2008.pdf 
Ministry of Transport. (2009). HOW NEW ZEALANDERS TRAVEL: Trends in New Zealand household travel 1989 - 2008. Retrieved from http://www.transport.govt.nz/assets/Import/Documents/How20New20Zealanders2 Otravel20web.pdf

Ministry of Transport. (2013a). New Zealand Household Travel Survey 2009-2012:

Comparing Travel Modes. Retrieved from

http://www.transport.govt.nz/assets/Import/Documents/NZ-househould-travelsurvey-Comparing-travel-modes-April-2013.pdf

Ministry of Transport. (2013b). The Social Cost of Road Crashes and Injuries, 2013

update. Wellington. Retrieved from

http://www.transport.govt.nz/assets/Uploads/Research/Documents/Social-Cost-ofRoad-Crashes-and-Injuries-June-2013-update.pdf

Muggeridge, D. (2012). Is Walking and Cycling Irresistible? Community Perceptions and Awareness of Active Travel in Hastings, New Zealand. Retrieved from http://researcharchive.vuw.ac.nz/handle/10063/2554

NZ Transport Agency. (2010). Economic evaluation manual. Wellington [N.Z.]: NZ Transport Agency.

NZTA. (2009a). National Land Transport Programme 2009-2012. Wellington. Retrieved from http://www.nzta.govt.nz/resources/national-land-transport-programme/20092012/docs/nltp-2009.pdf

NZTA. (2009b). National Land Transport Programme 2009-2012. Retrieved from http://www.nzta.govt.nz/resources/national-land-transport-programme/2009-2012/

NZTA. (2012). National Land Transport Programme 2012-15: At a Glance. Retrieved from http://www.nzta.govt.nz/resources/national-land-transport-programme/20122015/at-a-glance.html 
NZTA. (2013a). Economic Evaluation Manual. Retrieved from

http://www.nzta.govt.nz/resources/economic-evaluation-manual/economicevaluation-manual/index.html

NZTA. (2013b). The Walking and Cycling Model Community Story With New Plymouth \& Hastings. Retrieved from http://www.nzta.govt.nz/planning/process/doc/model-community-story-single.pdf

NZTA. (2013c). The Walking and Cycling Model Community Story with New Plymouth and Hastings. Retrieved from http://www.nzta.govt.nz/planning/process/doc/model-community-story-single.pdf

Ogilvie, D., Bull, F., Powell, J., Cooper, A. R., Brand, C., Mutrie, N., ... Rutter, H. (2011). An applied ecological framework for evaluating infrastructure to promote walking and cycling: the iConnect study. American Journal of Public Health, 101(3). Retrieved from http://search.ebscohost.com/login.aspx?direct=true\&profile=ehost\&scope=site\&au thtype $=$ crawler\&jrnl $=00900036 \&$ AN $=59439996 \& \mathrm{~h}=\mathrm{NPB}$ sf7 KDLZvQ5NhtuNi\%2 F1y\%2FyPQqnz2UohPuLm56taAJZoCJhFIpDXPjt0vjiPseqKl5dXfkbp\%2BS\%2 FnhQc1p\%2Bzww\%3D\%3D\&crl=c

Ogilvie, D., Egan, M., Hamilton, V., \& Petticrew, M. (2004). Promoting walking and cycling as an alternative to using cars: systematic review. BMJ, 329(7469), 763-0. doi:10.1136/bmj.38216.714560.55

Ogilvie, D., Mitchell, R., Mutrie, N., Petticrew, M., \& Platt, S. (2010). Research Shoe leather epidemiology: active travel and transport infrastructure in the urban landscape. Retrieved from http://www.biomedcentral.com/content/pdf/1479-58687-43.pdf 
Pettit, T. (2013). Deconstructing the New Zealand Transport Agency Economic

Evaluation Manual: Bringing the best research on transportation and its economic benefit into the applied New Zealand context. Victoria University of Wellington.

Preston, J. (2009). Epilogue: Transport policy and social exclusion-Some reflections.

Transport Policy, 16(3), 140-142. doi:10.1016/j.tranpol.2009.04.003

Pronk, N. P., Martinson, B., Kessler, R. C., Beck, A. L., Simon, G. E., \& Wang, P. (2004). The Association Between Work Performance and Physical Activity, Cardiorespiratory Fitness, and Obesity: Journal of Occupational and

Environmental Medicine, 46(1), 19-25.

doi:10.1097/01.jom.0000105910.69449.b7

Proper, K., \& van Mechelen, W. (2008). Effectiveness and economic impact of worksite interventions to promote physical activity and healthy diet. World Health Environmental. Retrieved from http://cdrwww.who.int/entity/dietphysicalactivity/Proper_K.pdf

Pucher, J., \& Buehler, R. (2008). Making Cycling Irresistible: Lessons from The Netherlands, Denmark and Germany. Transport Reviews, 28(4), 495-528. doi:10.1080/01441640701806612

Pucher, J., Dill, J., \& Handy, S. (2010). Infrastructure, programs, and policies to increase bicycling: An international review. Preventive Medicine, 50, S106-S125. doi:10.1016/j.ypmed.2009.07.028

Puentes, R. (2009). NFHS Policy Debate Topic Paper (Congressional Testimony presented before Committe on Banking, Housing and Urban Affairs). Washington, DC: Brookings Institution. Retrieved from http://www.brookings.edu/ /media/Research/Files/Testimony/2009/3/26\%20hous ing\%20puentes/0326_housing_puentes.PDF 
Quiggin, J. (1997). Discount rates and sustainability. International Journal of Social Economics, 24(1/2/3), 65-90.

Rietveld, P. (2000). Non-motorised modes in transport systems: a multimodal chain perspective for The Netherlands. Transportation Research Part D: Transport and Environment, 5(1), 31-36.

Roca, R., \& Crawford, L. (2013). 5 Reasons Why Bicycle Tourism Matters. Path Less Pedaled. Retrieved from http://pathlesspedaled.com/2013/03/5-reasons-whybicycle-tourism-matters/

Ruta, G. (2002). The social cost of transport. The World Bank Institute, Edición Mimeografiada. Retrieved from http://info.worldbank.org/etools/docs/library/36514/SocialCostTransport.pdf

Rutter, H., Cavill, N., Racioppi, F., Dinsdale, H., Oja, P., \& Kahlmeier, S. (2013). Economic Impact of Reduced Mortality Due to Increased Cycling. American Journal of Preventive Medicine, 44(1), 89-92. doi:10.1016/j.amepre.2012.09.053

Saelens, B. E., Sallis, J. F., \& Frank, L. D. (2003). Environmental correlates of walking and cycling: findings from the transportation, urban design, and planning literatures. Annals of Behavioral Medicine, 25(2), 80-91.

Saelensminde, K. (2004). Cost-benefit analyses of walking and cycling track networks taking into account insecurity, health effects and external costs of motorized traffic. Transportation Research Part A: Policy and Practice, 38(8), 593-606. doi:10.1016/j.tra.2004.04.003

Shiftan, Y., \& Shefer, D. (2009). Evaluating the impact of transport projects: Lessons for other disciplines. Evaluation and Program Planning, 32(4), 311-314. doi:10.1016/j.evalprogplan.2009.08.003 
Sloman, L., Cavill, N., Cope, A., Muller, L., \& Kennedy, A. (2009). Analysis and synthesis of evidence on the effects of investment in six Cycling Demonstration Towns. Report for Department for Transport and Cycling England.

SQW. (2006). Valuing the benefits of cycling: A report to Cycling England.

Stern, N. (2006). the Price of Change. IAEA BULLETIN, 48(2), 25.

Tin, S. T., Woodward, A., Thornley, S., \& Ameratunga, S. (2011). Regional variations in pedal cyclist injuries in New Zealand: safety in numbers or risk in scarcity? Australian and New Zealand Journal of Public Health, 35(4), 357-363. doi:10.1111/j.1753-6405.2011.00731.x

Tobias, M., Turley, M., New Zealand, \& Ministry of Health. (2013). Health loss in New Zealand: a report from the New Zealand burden of diseases, injuries and risk factors study, 2006-2016. Retrieved from https://www.health.govt.nz/system/files/documents/publications/health-loss-in-newzealand-final.pdf

UK Department for Transport. (2012). Guidance on the Appraisal of Walking and Cycling Schemes, TAG Unit 3.14.1. Retrieved from http://www.dft.gov.uk/webtag/documents/expert/unit3.14.php

UK Department for Transport. (2014). Introduction to Transport Analysis, TAG Unit 1.1. Retrieved from http://www.dft.gov.uk/webtag/documents/overview/unit1.1.php

UNICEF. (2009). State of the World's Children: Celebrating 20 Years of the Convention on the Rights of the Child. Unicef. Retrieved from http://books.google.com/books?hl=en\&lr=\&id=TsSsUZ990HUC\&oi=fnd\&pg=PA $1 \& \mathrm{dq}=\% 22 \mathrm{Cities}+$ Initiative $+(\mathrm{CFCI}) \% 22+\% 22$ of + global + societies,+ with + half $\% 22+$ $\% 22$ mission, + the + secretariat $\% 22+\% 22$ and + decent + sanitation, + and $\% 22+\% 22$ in + an + unpolluted + environment\%22+\%22population + now + living + in $\% 22+\% 22$ on + local + 
frameworks\%22+\&ots=uUzXJWDkwi\&sig=41qdZ6nATb1EM2hBQOU9zUCY

Xtw

United Nations Environment Programme. (2010). Share the Road: Investment in

Walking and Cycling Road Infrastructure. Nairobi. Retrieved from http://www.unep.org/transport/sharetheroad/PDF/SharetheRoadReportweb.pdf

Van Amelsvoort, L. G. P. M. (2006). Leisure time physical activity and sickness absenteeism; a prospective study. Occupational Medicine, 56(3), 210-212. doi:10.1093/occmed/kqj026

Van Goeverden, K., \& Godefrooij, T. (2011). The Dutch Reference Study: Cases of interventions in bicycle infrastructure reviewed in the framework of Bikeability. Deparment Transport \& Planning. Retrieved from www.repository.tudelft.nl/assets/uuid...6ebf.../Report\%20Dutch\%20cases.pdf

Veisten, K., Sælensminde, K., Alvær, K., Bjørnskau, T., Elvik, R., Schistad, T., \& Ytterstad, B. (2007). Total costs of bicycle injuries in Norway: Correcting injury figures and indicating data needs. Accident Analysis \& Prevention, 39(6), 11621169. doi:10.1016/j.aap.2007.03.002

Vreeker, R., Nijkamp, P., \& Ter Welle, C. (2002). A multicriteria decision support methodology for evaluating airport expansion plans. Transportation Research Part D: Transport and Environment, 7(1), 27-47.

Whitzman, C., Worthington, M., \& Mizrachi, D. (2010). The journey and the destination matter: Child-Friendly Cities and children's right to the City. Built Environment, 36(4), 474-486.

Wilson, A., \& Cope, A. (2011). VALUE FOR MONEY OF WALKING AND

CYCLING INTERVENTIONS: MAKING THE CASE FOR INVESTMENT

IN ACTIVE TRAVEL. Retrieved from

http://starconference.org.uk/star/2011/angelaWilson.pdf 
Winters, M., Davidson, G., Kao, D., \& Teschke, K. (2010). Motivators and deterrents of bicycling: comparing influences on decisions to ride. Transportation, 38(1), 153168. doi:10.1007/s11116-010-9284-y

Yeates, M. (1999). Integrating urban design: meeting the needs of people. Velo City Conference.

Yeates, M. (2001). PUTTING CYCLING ON THE TRANSPORT MAP ... A “COLLISION” BETWEEN FAST TRAFFIC AND URBAN DESIGN? VeloCity Conference. 
Appendix A Ethics Approval

TE WHARE WÄNANGA O TE ÖPOKO O TE IKA A MÃUT

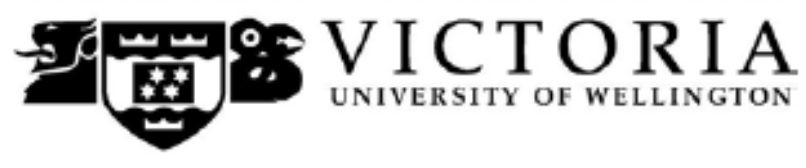

MEMORANDUM

Phone $\quad 0-4-4635676$

Fax $\quad 0-4-4635209$

Email Allison.kirkman@vuw.ac.nz

\begin{tabular}{l|l}
\hline TO & Mark Grams \\
\hline COPY TO & Ralph Chapman \\
\hline FROM & Dr Allison Kirkman, Convener, Human Ethics Committee \\
\hline
\end{tabular}

\begin{tabular}{l|l}
\hline DATE & 17 September 2013 \\
\hline PAGES & 1 \\
\hline
\end{tabular}

\begin{tabular}{l|l}
\hline SUBJECT & $\begin{array}{l}\text { Ethics Approval: } 20010 \\
\text { Active Investment: Evaluating the benefits and costs of investing in } \\
\text { active transport }\end{array}$ \\
\hline
\end{tabular}

Thank you for your application for ethical approval, which has now been considered by the Standing Committee of the Human Ethics Committee.

Your application has been approved from the above date and this approval continues until 4 August 2014. If your data collection is not completed by this date you should apply to the Human Ethics Committee for an extension to this approval.

Best wishes with the research.

Allison Kirkman

Human Ethics Committee

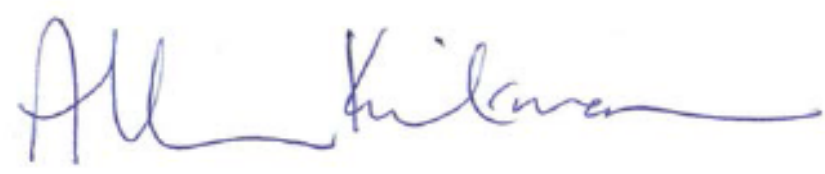


Appendix B MCP cost estimation

\begin{tabular}{|c|c|c|c|c|c|c|c|c|c|c|c|c|}
\hline & & Infrastruct & ture & & Education & Promotion & & Maintenar & nce & & & \\
\hline & year & NP & $\mathrm{H}$ & total & NP & $\mathrm{H}$ & total & NP & $\mathrm{H}$ & & & \\
\hline 2010 & 1 & 654696 & 2167000 & 2821696 & 334075 & 479000 & 813075 & 19641 & 30000 & & & \\
\hline 2011 & 2 & 1468147 & 3396000 & 4864147 & 754532 & 359000 & $1113532^{\prime}$ & 63685 & 40000 & Interventic & on period & \\
\hline 2012 & 3 & 2371762 & 117000 & 2488762 & 474933 & 259000 & 733933 & 134838 & 50000 & spending & & \\
\hline 2013 & 4 & & & & & & 587146 & 134838 & 50000 & & 12835145 & \\
\hline 2014 & 5 & & & & & & 469717 & 134838 & 50000 & & & \\
\hline 2015 & 6 & & & & & & 375774 & 134838 & 50000 & & & \\
\hline 2016 & 7 & & & & & & 300619 & 134838 & 50000 & & & \\
\hline 2017 & 8 & & & & & & 240495 & 134838 & 50000 & & & \\
\hline 2018 & 9 & & & & & & 192396 & 134838 & 50000 & & & \\
\hline 2019 & 10 & & & & & & 153917 & 134838 & 50000 & & & \\
\hline 2020 & 11 & & & & & & 123134 & 134838 & 50000 & & & \\
\hline 2021 & 12 & & & & & & 98507 & 134838 & 50000 & & & \\
\hline 2022 & 13 & & & & & & 78805 & 134838 & 50000 & & & \\
\hline 2023 & 14 & & & & & & 63044 & 134838 & 50000 & & & \\
\hline 2024 & 15 & & & & & & 50435 & 134838 & 50000 & & & \\
\hline 2025 & 16 & & & & & & 40348 & 134838 & 50000 & & & \\
\hline 2026 & 17 & & & & & & 32279 & 134838 & 50000 & & & \\
\hline 2027 & 18 & & & & & & 25823 & 134838 & 50000 & & & \\
\hline 2028 & 19 & & & & & & 20658 & 134838 & 50000 & & & \\
\hline 2029 & 20 & & & & & & 16527 & 134838 & 50000 & & & \\
\hline 2030 & 21 & & & & & & 13221 & 134838 & 50000 & & & \\
\hline 2031 & 22 & & & & & & 10577 & 134838 & 50000 & & & \\
\hline 2032 & 23 & & & & & & 8462 & 134838 & 50000 & & & \\
\hline 2033 & 24 & & & & & & 6769 & 134838 & 50000 & & & \\
\hline 2034 & 25 & & & & & & 5415 & 134838 & 50000 & & & \\
\hline 2035 & 26 & & & & & & 4332 & 134838 & 50000 & & & \\
\hline 2036 & 27 & & & & & & 3466 & 134838 & 50000 & & & \\
\hline 2037 & 28 & & & & & & 2773 & 134838 & 50000 & & & \\
\hline 2038 & 29 & & & & & & 2218 & 134838 & 50000 & & & \\
\hline 2039 & 30 & & & & & & 1775 & 134838 & 50000 & 30 years & 21092573 & \\
\hline 2040 & 31 & & & & & & 1420 & 134838 & 50000 & & & \\
\hline 2041 & 32 & & & & & & 1136 & 134838 & 50000 & & & \\
\hline 2042 & 33 & & & & & & 909 & 134838 & 50000 & & & \\
\hline 2043 & 34 & & & & & & 727 & 134838 & 50000 & & & \\
\hline 2044 & 35 & & & & & & 581 & 134838 & 50000 & & & \\
\hline 2045 & 36 & & & & & & 465 & 134838 & 50000 & & & \\
\hline 2046 & 37 & & & & & & 372 & 134838 & 50000 & & & \\
\hline 2047 & 38 & & & & & & 298 & 134838 & 50000 & & & \\
\hline 2048 & 39 & & & & & & 238 & 134838 & 50000 & & & \\
\hline 2049 & 40 & & & & & & 191 & 134838 & 50000 & 40 year c & 22947291 & \\
\hline 2050 & 41 & & & & & & 152 & 134838 & 50000 & & & \\
\hline 2051 & 42 & & & & & & 122 & 134838 & 50000 & & & \\
\hline 2052 & 43 & & & & & & 98 & 134838 & 50000 & & & \\
\hline 2053 & 44 & & & & & & 78 & 134838 & 50000 & & & \\
\hline 2054 & 45 & & & & & & 62 & 134838 & 50000 & & & \\
\hline 2055 & 46 & & & & & & 50 & 134838 & 50000 & & & \\
\hline 2056 & 47 & & & & & & 40 & 134838 & 50000 & & & \\
\hline 2057 & 48 & & & & & & 32 & 134838 & 50000 & & & \\
\hline 2058 & 49 & & & & & & 26 & 134838 & 50000 & & & \\
\hline 2059 & 50 & & & & & & 20 & 134838 & 50000 & 50 year c & 24796353 & \\
\hline
\end{tabular}


Appendix C Active travel growth estimates

\begin{tabular}{|c|c|c|c|c|c|c|c|c|c|c|}
\hline & & \multicolumn{9}{|c|}{ Growthrates \% } \\
\hline & & \multicolumn{3}{|c|}{ Walking (Intervention) } & \multicolumn{3}{|c|}{ Cycling (Intervention) } & \multicolumn{3}{|l|}{ (Control) } \\
\hline & & Medium & High & Low & Medium & High & Low & Walking & Cycling & 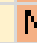 \\
\hline 2009 & baseline & 2.22 & 2.22 & 2.22 & -1.23 & -1.23 & -1.23 & -2.53 & -1.94 & \\
\hline 2010 & 1 & -1.65 & -1.65 & -1.65 & 5.18 & 5.18 & 5.18 & -2.53 & -1.94 & \\
\hline 2011 & 2 & -1.65 & -1.65 & -1.65 & 5.18 & 5.18 & 5.18 & -2.53 & -1.94 & \\
\hline 2012 & 3 & -1.65 & -1.65 & -1.65 & 5.18 & 5.18 & 5.18 & -2.53 & -1.94 & \\
\hline 2013 & 4 & -1.65 & -1.65 & -1.65 & 5.18 & 5.18 & 5.18 & -2.53 & -1.94 & \\
\hline 2014 & 5 & -1.65 & -1.65 & -1.65 & 5.18 & 5.18 & 5.18 & -2.53 & -1.94 & \\
\hline 2015 & 6 & -1.65 & -1.65 & -1.65 & 3.62 & 4.14 & 3.11 & -2.53 & -1.94 & \\
\hline 2016 & 7 & -1.65 & -1.65 & -1.65 & 2.54 & 3.31 & 1.86 & -2.53 & -1.94 & \\
\hline 2017 & 8 & -1.65 & -1.65 & -1.65 & 1.78 & 2.65 & 1.12 & -2.53 & -1.94 & \\
\hline 2018 & 9 & -1.65 & -1.65 & -1.65 & 1.24 & 2.12 & 0.67 & -2.53 & -1.94 & \\
\hline 2019 & 10 & -1.65 & -1.65 & -1.65 & 0.87 & 1.70 & 0.40 & -2.53 & -1.94 & \\
\hline 2020 & 11 & -1.65 & -1.65 & -1.65 & 0.61 & 1.36 & 0.24 & -2.53 & -1.94 & \\
\hline 2021 & 12 & -1.65 & -1.65 & -1.65 & 0.43 & 1.09 & 0.14 & -2.53 & -1.94 & \\
\hline 2022 & 13 & -1.65 & -1.65 & -1.65 & 0.30 & 0.87 & 0.09 & -2.53 & -1.94 & \\
\hline 2023 & 14 & -1.65 & -1.65 & -1.65 & 0.21 & 0.69 & 0.05 & -2.53 & -1.94 & \\
\hline 2024 & 15 & -1.65 & -1.65 & -1.65 & 0.15 & 0.56 & 0.03 & -2.53 & -1.94 & \\
\hline 2025 & 16 & -1.65 & -1.65 & -1.65 & 0.15 & 0.56 & 0.03 & -2.53 & -1.94 & \\
\hline 2026 & 17 & -1.65 & -1.65 & -1.65 & 0.15 & 0.56 & 0.03 & -2.53 & -1.94 & \\
\hline 2027 & 18 & -1.65 & -1.65 & -1.65 & 0.15 & 0.56 & 0.03 & -2.53 & -1.94 & \\
\hline 2028 & 19 & -1.65 & -1.65 & -1.65 & 0.15 & 0.56 & 0.03 & -2.53 & -1.94 & \\
\hline 2029 & 20 & -1.65 & -1.65 & -1.65 & 0.15 & 0.56 & 0.03 & -2.53 & -1.94 & \\
\hline 2030 & 21 & -1.65 & -1.65 & -1.65 & 0.15 & 0.56 & 0.03 & -2.53 & -1.94 & \\
\hline 2031 & 22 & -1.65 & -1.65 & -1.65 & 0.15 & 0.56 & 0.03 & -2.53 & -1.94 & \\
\hline 2032 & 23 & -1.65 & -1.65 & -1.65 & 0.15 & 0.56 & 0.03 & -2.53 & -1.94 & \\
\hline 2033 & 24 & -1.65 & -1.65 & -1.65 & 0.15 & 0.56 & 0.03 & -2.53 & -1.94 & \\
\hline 2034 & 25 & -1.65 & -1.65 & -1.65 & 0.15 & 0.56 & 0.03 & -2.53 & -1.94 & \\
\hline 2035 & 26 & -1.65 & -1.65 & -1.65 & 0.15 & 0.56 & 0.03 & -2.53 & -1.94 & \\
\hline 2036 & 27 & -1.65 & -1.65 & -1.65 & 0.15 & 0.56 & 0.03 & -2.53 & -1.94 & \\
\hline 2037 & 28 & -1.65 & -1.65 & -1.65 & 0.15 & 0.56 & 0.03 & -2.53 & -1.94 & \\
\hline 2038 & 29 & -1.65 & -1.65 & -1.65 & 0.15 & 0.56 & 0.03 & -2.53 & -1.94 & \\
\hline 2039 & 30 & -1.65 & -1.65 & -1.65 & 0.15 & 0.56 & 0.03 & -2.53 & -1.94 & \\
\hline 2040 & 31 & -1.65 & -1.65 & -1.65 & 0.15 & 0.56 & 0.03 & -2.53 & -1.94 & \\
\hline 2041 & 32 & -1.65 & -1.65 & -1.65 & 0.15 & 0.56 & 0.03 & -2.53 & -1.94 & \\
\hline 2042 & 33 & -1.65 & -1.65 & -1.65 & 0.15 & 0.56 & 0.03 & -2.53 & -1.94 & \\
\hline 2043 & 34 & -1.65 & -1.65 & -1.65 & 0.15 & 0.56 & 0.03 & -2.53 & -1.94 & \\
\hline 2044 & 35 & -1.65 & -1.65 & -1.65 & 0.15 & 0.56 & 0.03 & -2.53 & -1.94 & \\
\hline 2045 & 36 & -1.65 & -1.65 & -1.65 & 0.15 & 0.56 & 0.03 & -2.53 & -1.94 & \\
\hline 2046 & 37 & -1.65 & -1.65 & -1.65 & 0.15 & 0.56 & 0.03 & -2.53 & -1.94 & \\
\hline 2047 & 38 & -1.65 & -1.65 & -1.65 & 0.15 & 0.56 & 0.03 & -2.53 & -1.94 & \\
\hline 2048 & 39 & -1.65 & -1.65 & -1.65 & 0.15 & 0.56 & 0.03 & -2.53 & -1.94 & \\
\hline 2049 & 40 & -1.65 & -1.65 & -1.65 & 0.15 & 0.56 & 0.03 & -2.53 & -1.94 & \\
\hline 2050 & 41 & -1.65 & -1.65 & -1.65 & 0.15 & 0.56 & 0.03 & -2.53 & -1.94 & \\
\hline 2051 & 42 & -1.65 & -1.65 & -1.65 & 0.15 & 0.56 & 0.03 & -2.53 & -1.94 & \\
\hline 2052 & 43 & -1.65 & -1.65 & -1.65 & 0.15 & 0.56 & 0.03 & -2.53 & -1.94 & \\
\hline 2053 & 44 & -1.65 & -1.65 & -1.65 & 0.15 & 0.56 & 0.03 & -2.53 & -1.94 & \\
\hline 2054 & 45 & -1.65 & -1.65 & -1.65 & 0.15 & 0.56 & 0.03 & -2.53 & -1.94 & \\
\hline 2055 & 46 & -1.65 & -1.65 & -1.65 & 0.15 & 0.56 & 0.03 & -2.53 & -1.94 & \\
\hline 2056 & 47 & -1.65 & -1.65 & -1.65 & 0.15 & 0.56 & 0.03 & -2.53 & -1.94 & \\
\hline 2057 & 48 & -1.65 & -1.65 & -1.65 & 0.15 & 0.56 & 0.03 & -2.53 & -1.94 & \\
\hline 2058 & 49 & -1.65 & -1.65 & -1.65 & 0.15 & 0.56 & 0.03 & -2.53 & -1.94 & \\
\hline 2059 & 50 & -1.65 & -1.65 & -1.65 & 0.15 & 0.56 & 0.03 & -2.53 & -1.94 & \\
\hline
\end{tabular}




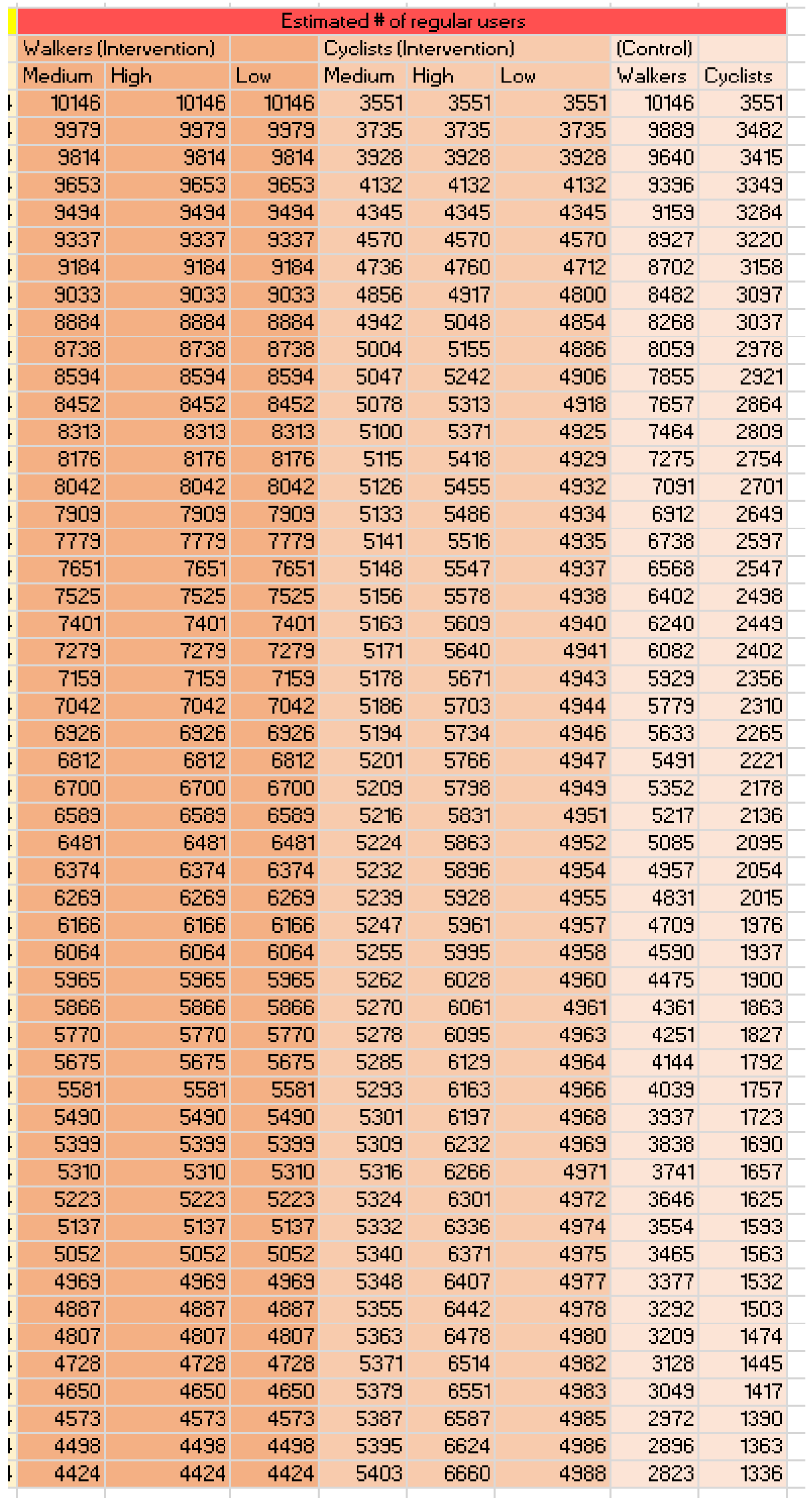


Appendix D UK Department for Transport Carbon Prices

\begin{tabular}{|c|c|c|c|c|c|c|}
\hline & \multicolumn{3}{|c|}{ Traded } & \multicolumn{3}{|c|}{ Non-traded } \\
\hline & Low & Central & High & Low & Central & High \\
\hline 2009 & 12.12 & 12.12 & 12.12 & 26.43 & 52.86 & 79.29 \\
\hline 2010 & 12.41 & 12.41 & 12.41 & 26.79 & 53.58 & 80.37 \\
\hline 2011 & 6.26 & 13.16 & 17.01 & 27.19 & 54.39 & 81.58 \\
\hline 2012 & 7.00 & 14.17 & 18.08 & 27.60 & 55.20 & 82.80 \\
\hline 2013 & 8.60 & 15.54 & 19.51 & 28.02 & 56.03 & 84.05 \\
\hline 2014 & 9.75 & 16.70 & 20.83 & 28.44 & 56.87 & 85.31 \\
\hline 2015 & 11.24 & 18.46 & 22.99 & 28.86 & 57.72 & 86.59 \\
\hline 2016 & 13.49 & 20.05 & 25.93 & 29.29 & 58.59 & 87.88 \\
\hline 2017 & 14.74 & 21.41 & 27.20 & 29.73 & 59.47 & 89.20 \\
\hline 2018 & 15.50 & 22.98 & 30.10 & 30.18 & 60.36 & 90.54 \\
\hline 2019 & 16.81 & 25.13 & 32.00 & 30.63 & 61.27 & 91.90 \\
\hline 2020 & 18.72 & 27.89 & 34.68 & 31.09 & 62.18 & 93.28 \\
\hline 2021 & 20.47 & 32.36 & 42.09 & 31.61 & 63.22 & 94.83 \\
\hline 2022 & 22.23 & 36.82 & 49.51 & 32.13 & 64.26 & 96.39 \\
\hline 2023 & 23.98 & 41.29 & 56.92 & 32.65 & 65.29 & 97.94 \\
\hline 2024 & 25.74 & 45.75 & 64.34 & 33.17 & 66.33 & 99.50 \\
\hline 2025 & 27.50 & 50.22 & 71.75 & 33.68 & 67.37 & 101.05 \\
\hline 2026 & 29.25 & 54.69 & 79.17 & 34.20 & 68.40 & 102.60 \\
\hline 2027 & 31.01 & 59.15 & 86.58 & 34.72 & 69.44 & 104.16 \\
\hline 2028 & 32.76 & 63.62 & 93.99 & 35.24 & 70.48 & 105.71 \\
\hline 2029 & 34.52 & 68.08 & 101.41 & 35.76 & 71.51 & 107.27 \\
\hline 2030 & 36.27 & 72.55 & 108.82 & 36.27 & 72.55 & 108.82 \\
\hline 2031 & 39.64 & 79.29 & 118.93 & 39.64 & 79.29 & 118.93 \\
\hline 2032 & 43.01 & 86.02 & 129.03 & 43.01 & 86.02 & 129.03 \\
\hline 2033 & 46.38 & 92.76 & 139.14 & 46.38 & 92.76 & 139.14 \\
\hline 2034 & 49.75 & 99.50 & 149.24 & 49.75 & 99.50 & 149.24 \\
\hline 2035 & 53.12 & 106.23 & 159.35 & 53.12 & 106.23 & 159.35 \\
\hline 2036 & 56.48 & 112.97 & 169.45 & 56.48 & 112.97 & 169.45 \\
\hline 2037 & 59.85 & 119.71 & 179.56 & 59.85 & 119.71 & 179.56 \\
\hline 2038 & 63.22 & 126.44 & 189.66 & 63.22 & 126.44 & 189.66 \\
\hline 2039 & 66.59 & 133.18 & 199.77 & 66.59 & 133.18 & 199.77 \\
\hline 2040 & 69.96 & 139.92 & 209.87 & 69.96 & 139.92 & 209.87 \\
\hline 2041 & 73.33 & 146.65 & 219.98 & 73.33 & 146.65 & 219.98 \\
\hline 2042 & 76.69 & 153.39 & 230.08 & 76.69 & 153.39 & 230.08 \\
\hline 2043 & 80.06 & 160.13 & 240.19 & 80.06 & 160.13 & 240.19 \\
\hline 2044 & 83.43 & 166.86 & 250.29 & 83.43 & 166.86 & 250.29 \\
\hline 2045 & 86.80 & 173.60 & 260.40 & 86.80 & 173.60 & 260.40 \\
\hline 2046 & 90.17 & 180.34 & 270.50 & 90.17 & 180.34 & 270.50 \\
\hline 2047 & 93.54 & 187.07 & 280.61 & 93.54 & 187.07 & 280.61 \\
\hline 2048 & 96.90 & 193.81 & 290.71 & 96.90 & 193.81 & 290.71 \\
\hline 2049 & 100.27 & 200.55 & 300.82 & 100.27 & 200.55 & 300.82 \\
\hline 2050 & 103.64 & 207.28 & 310.92 & 103.64 & 207.28 & 310.92 \\
\hline 2051 & 106.23 & 214.61 & 322.99 & 106.23 & 214.61 & 322.99 \\
\hline 2052 & 108.64 & 221.71 & 334.79 & 108.64 & 221.71 & 334.79 \\
\hline 2053 & 110.99 & 228.85 & 346.71 & 110.99 & 228.85 & 346.71 \\
\hline 2054 & 113.27 & 235.99 & 358.70 & 113.27 & 235.99 & 358.70 \\
\hline 2055 & 115.38 & 242.90 & 370.42 & 115.38 & 242.90 & 370.42 \\
\hline 2056 & 117.43 & 249.85 & 382.27 & 117.43 & 249.85 & 382.27 \\
\hline 2057 & 119.30 & 256.55 & 393.80 & 119.30 & 256.55 & 393.80 \\
\hline 2058 & 121.02 & 263.09 & 405.16 & 121.02 & 263.09 & 405.16 \\
\hline 2059 & 122.63 & 269.52 & 416.41 & 122.63 & 269.52 & 416.41 \\
\hline
\end{tabular}

DfT (2012, p.7-8) 
Appendix E Intercept survey questions for iWay users (note, questions are identical for Let's Go users)

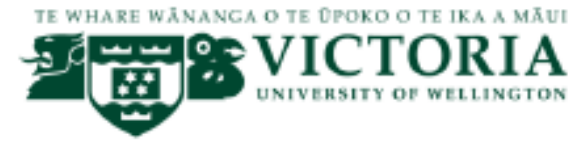

Interview questions, Hastings walking and cycling

\section{(1.1) Sustainable Cities}

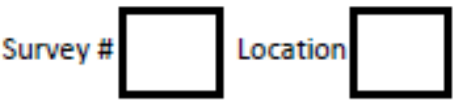

Date

The following questions are about your experience and knowledge as a user of walking and cycling facilities in Hastings.

1) Are you aware of the iWay programme in Hastings?

2) a) Did you regularly walk (on average at least once a week for at least 10 minutes at a time) in the Hastings area in 2009, the year prior to the start of the iWay programme, for any purpose? [lf answer is no, go to question 4]

b) If so, please give an estimate of distance or time you would have walked in an average week (in kilometres or hours)?

3) Please rate the quality of Hastings area walking facilities area in 2009, the year prior to the start of the iWay programme, on the following criteria: (on a scale of $1-10,10$ being the highest)
a) Personal safety (lighting etc...)
b) Surface quality (smoothness, not muddy etc..)
c) Separation from motor vehicles
d) Coverage (routes take you directly where you want to go)
e) Enjoyment
f) Positive motorist attitudes towards walkers 
4) a) Turning to cycling, did you regularly cycle (on average at least once a week) in the Hastings area in 2009, the year prior to the start of the iWay programme, for any purpose? [lf answer is no, go to question 6]

b) If so, please give an estimate for distance or time you would you have cycled in an average week (in kilometres or hours)?

5) Please rate the quality of Hastings area cycling facilities in 2009, the year prior to the start of the iWay programme, on the following criteria: (on a scale of 1-10, 10 being the highest)

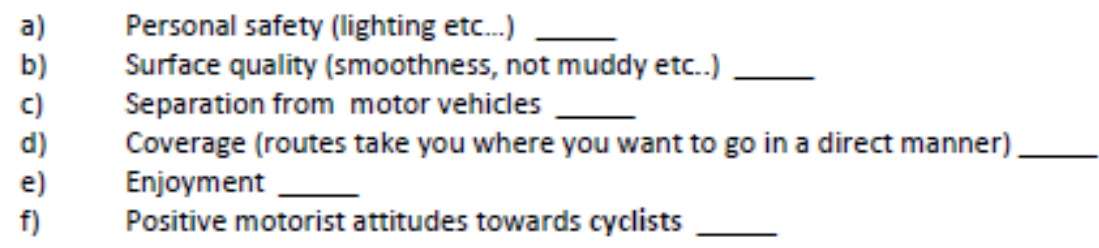

6) a) Since the start of 2013 , have you regularly walked in the Hastings area (on average at least once a week for at least 10 minutes at a time) for any purpose? [if answer is no, go to question 8]

b) If so, please give an estimate of distance or time you have you been walking in an average week (in kilometres or hours)?

7) Please rate the quality of Hastings area walking facilities since the start of 2013 on the following criteria: (on a scale of 1-10, 10 being the highest)

a) Personal safety (lighting etc...)

b) Surface quality (smoothness, not muddy etc..)

c) Separation from motor vehicles

d) Coverage (routes take you directly where you want to go)

e) Enjoyment

f) Positive motorist attitudes towards walkers 
8) a) Since the start of 2013 , have you regularly cycled in the Hastings area (on average at least once a week)? [lf answer is no, go to question 10]

b) If so, approximately how many kilometres have you been cycling in an average week?

9) Please rate the quality of Hastings area cycling facilities since the start of 2013 on the following criteria: (on a scale of $1-10,10$ being the highest)
a) Personal safety (lighting etc...)
b) Surface quality (smoothness, not muddy etc..)
c) Separation from motor vehicles
d) Coverage (routes take you where you want to go in a direct manner)
e) Enjoyment
f) Positive motorist attitudes towards cyclists

10) I'd like to focus on walking once again, this time thinking about what's important to you in regard to improving walking facilities. Please rank the following factors in order from 1 to 6 , 1 being the most important factor for improving walking facilities, and 6 being the least important:
a) Personal safety (lighting etc...)
b) Surface quality (smoothness, not muddy etc..)
c) Separation from motor vehicles
d) Coverage (routes take you directly where you want to go)
e) Enjoyment
f) Positive motorist attitudes towards walkers

11) Now focusing on cycling: Please rank the following factors in order from 1 to 6,1 being the most important factor for you for improving cycling facilities, and 6 being the least important:

\footnotetext{
a) Personal safety (lighting etc...)

b) Surface quality (smoothness, not muddy etc..)

c) Separation from motor vehicles

d) Coverage (routes take you where you want to go in a direct manner)

e) Enjoyment

f) Positive motorist attitudes towards cyclists
} 
12) As a walker/cyclists in Hastings, what value (\$ per year) would you place on walking and cycling facilities in the Hastings area?

$$
(\$ 0-\$ 50) \quad(\$ 51-\$ 100) \quad(\$ 101-\$ 200) \quad(\$ 201-\$ 500) \quad(>\$ 500)
$$

13) In your own words, briefly describe how the iWay programme (improvements in walking/cycling infrastructure and promotion) has impacted your lifestyle, if at all.

14) In your own words, briefly describe how the improved walking and cycling infrastructure built in the past three years has changed your transportation habits, if at all.

15) In your own words, briefly describe how the improved walking and cycling infrastructure built in the past three years is likely to affect your transportation habits in the next five years, if at all.

16) In your own words, briefly describe any way in which the improved walking and cycling infrastructure built in the last three years has affected your community within the Hastings District. 


\section{Demographics}

(for statistical purposes, circle relevant responses)

1) Male / Female

2) Age bracket:

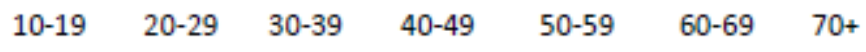

3) Which ethnicity best describes you (you may pick more than one)

Maori European Pacific Islander Asian Other

4) What is your home address (for GIS analysis, you may provide the nearest street corner if you wish)? 
Appendix F Project manager interview questions

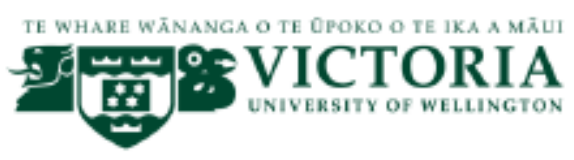

\title{
(iii) Sustainable Cities
}

\author{
Interview Questions, Model Communities Programme, Project Managers
}

The following questions seek information about your knowledge and experience as project managers of the Model Communities Programme in either Hastings or New Plymouth. The term "programme" refers to the Model Communities Programme run by your district council.

(i) How much of the initial programme was funded by NZTA (\$ by year)?

(a) How much was provided by the district council?

(b) Were there any other sources of funding for the programme, and if so, how much ( $\$$ by year)? "this excludes complimentary but separate external programmes

(ii) How much of these costs were council workers' salaries who would have otherwise been employed by the council outside of this project?

(a) Were the costs of diverted staff included in the district council's costs in question i(a) above?

(iii) How much of expenditure ( $\$$ by year) went to building and improving infrastructure?

(a) How much expenditure went to educational and promotional initiatives?

(b) Can you give a brief breakdown of the types of infrastructure built, preferably differentiating between walking infrastructure and cycling infrastructure?

(c) What specifically was promotion and educational expenditure spent on? 
(iv) Were any components of the programme unexpectedly challenging?

(a) Were any components of the programme more expensive than expected?

(b) Were any components of the programme less costly than expected?

(v) Which socio-demographic group does the council believe has benefitted the most from this project? (eg age, gender, income, ethnic and other groups)

(a) Is there a group which has so far benefitted less than most?

(vi) How is the programme expanding beyond the initial three year funding period?

(a) How will this future funding be divided between NZTA and the district council ( $\$$ by year)?

(b) How will future funding be divided between infrastructure and education/promotion?

(vii) Upon completion of the programme, what do you expect yearly maintenance costs of the infrastructure to be?

(viii) What are the district council's goals for promoting active transport upon completion of the project and do they differ from existing programme goals?

(a) How much expenditure is budgeted for promoting active transport in the years after the end of the project?

(ix) In hindsight, if you were starting afresh promoting active transport in this city, how would you approach it differently this time around?

(x) What would you identify as particular successes and weaknesses of the Model Communities Programme in your district to date? 
(xi) What do you see as the pros and cons of cost-benefit analysis as a means of assessing the effectiveness of the Model Communities programme in your district?

(a) In your opinion, what are the attractions of cost-benefit analysis in evaluating the overall merits of your programme?

(b) In your opinion, what are the limitations of cost-benefit analysis in evaluating the overall merits of your programme?

(xii) How do you envision the evolution of walking and cycling within your district council in the next thirty years?

(a) What's a realistic modal share for walking and cycling trips within your district? 